\title{
RHEOLOGY OF WEAKLY ATTRACTIVE SOFT PARTICLES
}

\author{
Dissertation \\ zur Erlangung des mathematisch-naturwissenschaftlichen \\ Doktorgrades \\ "Doctor rerum naturalium" (Dr.rer.nat.) \\ der Georg-August-Universität Göttingen \\ im Promotionsprogramm ProPhys \\ der Georg-August University School of Science (GAUSS)
}

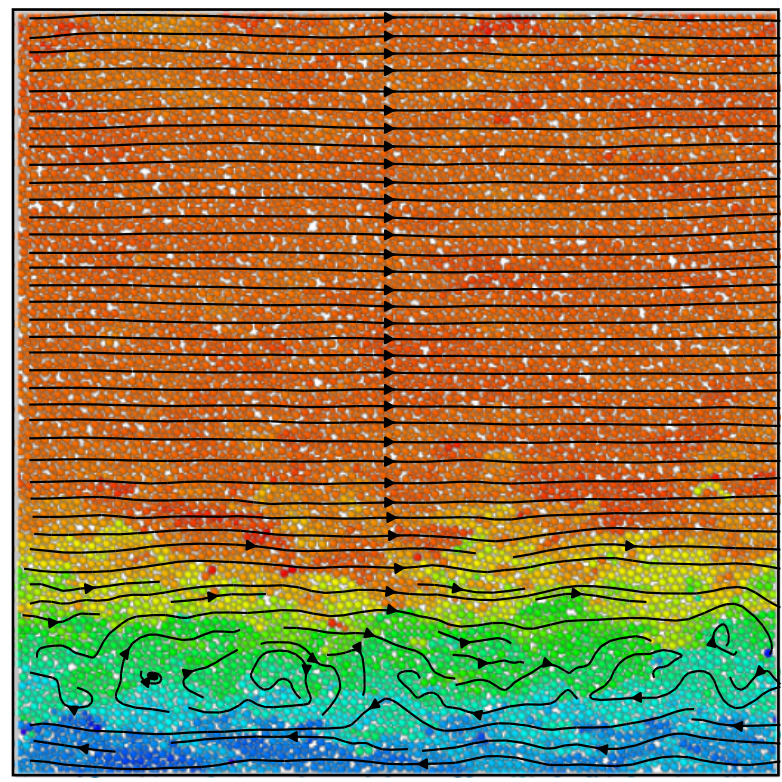

\author{
vorgelegt von \\ Ehsan Irani \\ aus Teheran, IRAN
}

Göttingen, July 2016 
- Dr. Claus Heussinger

Institut für Theoretische Physik, Georg-August-Universität Göttingen

- Prof. Dr. Annette Zippelius

Institut für Theoretische Physik, Georg-August-Universität Göttingen

MEMBERS OF THE EXAMINATION BOARD:

REVIEWERS

- Dr. Claus Heussinger, Institut für Theoretische Physik, Georg-August-Universität Göttingen

- Prof. Dr. Annette Zippelius Institut für Theoretische Physik, Georg-August-Universität Göttingen

FURTHER MEMBERS OF THE EXAMINATION BOARD

- Dr. Pinaki Caudhuri Institute of Mathematical Sciences, Chennai, India

- Prof. Dr. Stephan Herminghaus

Dynamik Komplexer Fluide, Max-Planck-Institut für Dynamik und Selbstorganisation

- Prof. Dr. Reiner Kree Institut für Theoretische Physik, Georg-August-Universität Göttingen

- Prof. Cynthia A. Volkert, PhD Institut für Materialphysik, Georg-August-Universität Göttingen

DISPUTATION : 10 August 2016

Ehsan Irani: Rheology of Weakly Attractive Soft Particles, , (C) July 2016 
Dedicated to my parents, Akram and Mohammad. 



\section{A BSTRACT}

We study the rheology of a soft particulate system with attractive interactions. Lees-Edwards boundary conditions are used to simulate a shear-controlled flow. Unlike repulsive systems, it is found that in systems with a damping force directed normally to the contact point, attractive interactions result in a finite yield stress, and an iso-static structure emerges below the jamming point. The rheology can be explained by a scaling argument that exploits the vicinity to the isostatic state. In addition, flow curves exhibit non-monotonic behavior, resulting in persistent shear-banding in large systems.

Furthermore we investigate the role of dissipation mechanism by implementing several models for the dissipation of energy. A tangential damping gives rise to the monotonic flow curves and the development of a viscous flow in the over-damped regime. However in that case, decreasing the damping factor introduces the inertial time-scale, leading again to non-monotonic flow curves and inertia-induced shear-banding, which are intrinsically different from the above mentioned shear bands.

Finally we introduce thermal fluctuations to our system and investigate the interplay of temperature and attraction with respect to flow properties and particles' dynamics. Namely a phase-separation at intermediate values of the underlying parameters is observed to occurs, the amount and rate of which has been quantified in this work by introducing a properly chosen order parameter.

Our results shed some light on the rich and complex rheological response of attractive particles, in terms of interaction details, such as the dissipation model, thermal noise and range of the attraction. 



\section{PUBLICATIONS}

Some results and figures presented in Chapters 3 and 4, have appeared previously in the following publications:

Irani, Ehsan, Pinaki Chaudhuri, and Claus Heussinger (2014). "Impact of Attractive Interactions on the Rheology of Dense Athermal Particles." In: Phys. Rev. Lett. 112 (18), p. 188303. Dor: 10. 1103/PhysRevLett. 112.188303. URL: http://link. aps . org/doi/ 10.1103/PhysRevLett.112.188303.

Irani, Ehsan, P. Chaudhuri, and C. Heussinger (2016). "Athermal rheology of weakly attractive soft particles." In: Phys. Rev. E 94 (5), p. 052608. DOI: 10.1103/PhysRevE. 94.052608. URL: http://link. aps.org/doi/10.1103/PhysRevE.94.052608. 

Different roads sometimes lead to the same castle.

Who knows?

- Jon Snow to Arya

A GAME OF THRONES

\section{ACKNOWLEDGEMENTS}

At the end of this long journey, I am glad to use this opportunity to thank people who were with me.

First of all, I would like to thank my supervisor, Dr. Claus Heussinger, who has granted me an excellent scientific guidance, as well as a great balance between supervision and freedom. His patience, sharp scientific insight and dedication to help, is all I could hope for, as a $\mathrm{PhD}$ student. I really appreciate all the time he patiently gave to me during our meetings in his place, when he was on leave. I am also grateful to Dr. Pinaki Chaudhuri, because of the fruitful collaboration during this project. I benefit a lot from all email-communications and Skype meetings that we had. His comments and ideas have always been crucially helpful to me. I would also like to thank Prof. Annette Zippelius for providing a promising scientific atmosphere in the "Statistical Physics and Complex Systems" group, for nice scientific and non-scientific discussions we had, and specially for her hints regarding the shear-banding in systems with full velocity dissipation.

I should sincerely thank Prof. Stephan Herminghaus, Prof. Reiner Kree and Prof. Cynthia Volkert who have accepted to be in my Examination Committee.

I am grateful to my beloved wife and colleague, Elham, who has been beside me in this long adventure. I enjoyed a lot from our scientific discussions. I appreciate her efforts in proofreading my thesis, and also all non-scientific supports she granted me.

During my PhD project, I had a memorable time in the institute for theoretical physics, am indebted to many individuals that I am glad to take this opportunity to thank. Specially Dr. Moumita Maiti because of our interesting discussions, (to be soon Dr.) Matthias Grob for persistent discussions and also his proofreading of Chapter 4, Dr. Sina Sadeghi, whom I enjoyed to share an office with for more than two years. I would also like to thank my other friends from the institute, Dr. Timo Fisher for his help in my early days in Göttingen, and people from the Hard Condensed Matter group, Stephan, Salvatore, 
Ebad, Benjamin, Johannes, Ingo and Fabian, and others. We had great activities, such as weekly movie-nights and football sessions. I would always remember these days joyfully.

A big thank goes to the secreteries, Katrin Glormann, Gabriel Schubert and Kati Oldenburg, for all the administrative support.

I am pleased that I had the chance to work with Prof. Thomas Pruschke twice, as a tutor of his lectures. I indeed learned a lot from that experience. Hereby, I would like to acknowledge him for all that. His memories will stay with the people from the institute.

I could have not finished this work without using of many nice opensource tools. I thank the open-source community, specially all developers involved in projects: g++, vim, gnuplot, python, Jupyter, TiddlyWiki, Atom, LAMMPS, ovito, Inkscape and for sure, GNU/Linux.

Last but not least, I am very grateful to my family, specially my parents, for all they have done for me these years. 


\section{CONTENTS}

I INTRODUCTORY MATERIAL

1 THEORETICAL BACKGROUND 3

1.1 Soft Material ............... 3

1.2 Categories of Soft Matter: Mechanical Response . . . . 4

1.2.1 Stress Tensor and Strain ........... 4

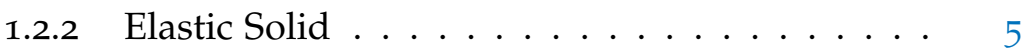

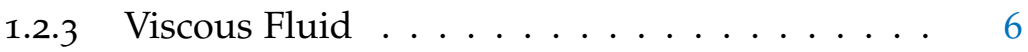

1.2.4 Newtonian Fluid ............ 6

1.2.5 Complex Fluid . . . . . . . . . . . 7

1.3 Rheology: Study of Flow and Deformation . . . . . . 9

1.3.1 Shear Geometries ............. 10

1.4 The Jamming Transition ............. 11

1.4.1 The Value of $\phi_{\mathrm{J}} \ldots \ldots \ldots 12$

1.5 Effect of Temperature ............ 13

1.5.1 The Glass Transition . . . . . . . . . . 13

1.5.2 Jamming Transition versus Glass Tansition ... 13

1.6 Role of Particles Interactions . . . . . . . . . . . 14

1.6.1 Dissipation and Inertia .......... 14

1.6.2 Friction ............... 16

1.6.3 Attractive Interactions . . . . . . . . . 17

1.7 Flow Heterogenities. . . . . . . . . . . . 18

2 Simulations: TECHNiCAL DETAils 21

2.1 Plane Shear: Lees-Edward Boundary Condition . . . . 21

2.1.1 Imposing Large Deformations . . . . . . . 22

2.2 Observables ................... 22

2.2.1 Stress Tensor . . . . . . . . . . . 22

2.2.2 Potential Energy ............ 23

2.3 Measurments ............... 23

II RESULTS 25

3 RHEOLOGY OF ATHERMAL SYSTEMS WITH WEAKLY ATTRACTIVE INTERACTIONS 27

3.1 The Particle Interactions . . . . . . . . . . . 27

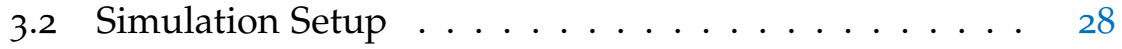

3.3 Flow Curves ................. 29

3.3.1 Finite Yield Stress ............ 31

3.3.2 Non-monotonic behavior ........ 31

3.4 Phase Diagram ............. 32

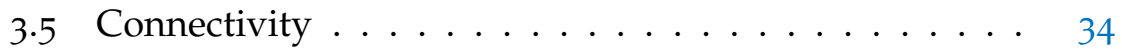

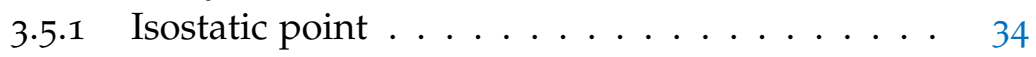

3.5.2 New Type of Connectivity ......... 35 
3.5.3 Rigidity Below the Jamming Point . . . . . . 36

3.5.4 Fragile Solid: Yield Stress and Connectivity . . 37

3.6 Potential Energy . . . . . . . . . . . . . . . . . . 40

3.7 Shear Stress Ratio . . . . . . . . . . . . . . . . 42

3.8 Structure Factor . . . . . . . . . . . . . . . 43

3.9 Particles Dynamics . . . . . . . . . . . . . . 46

3.9.1 Mean-squared Displacement . . . . . . . . 46

3.9 .2 Velocity Fluctuations . . . . . . . . . . . 50

3.10 Consitutive Relation: The Fluidity Model Approach . . 51

3.11 Shear Bands: Theory . . . . . . . . . . . . . 53

3.11.1 Constitutive Equation . . . . . . . . . . . 53

3.11 .2 Van der Waals Loop . . . . . . . . . . . . . 54

3.11.3 Maxwell Construction and Phase Coexistence . 56

3.11.4 Linear Stability Analysis: Size Matters . . . . . . 57

3.12 Shear Bands: Simulations . . . . . . . . . . 58

3.12.1 The form of $k(\dot{\gamma}) \ldots \ldots \ldots \ldots$

3.12.2 Flow Curves and System Size . . . . . . . . . . 59

3.12.3 Properties of Shear Bands . . . . . . . . . 60

4 THE ROLE OF DisSiPATION 63

4.1 Introduction . . . . . . . . . . . 63

4.2 Damping Regimes . . . . . . . . . . . . . 63

4.2.1 Over-damped Oscillator . . . . . . . . . . . 65

4.2.2 Under-damped Oscillator . . . . . . . . . . . 65

4.2.3 Critical Damping . . . . . . . . . . . . . . 65

4.3 Different Damping Mechanisms . . . . . . . . 66

4.4 Dissipation in Normal Direction . . . . . . . . . . . 67

4.5 Full Contact Dissipation . . . . . . . . . . . . . 69

4.5 .1 Flow Curves . . . . . . . . . . 70

4.5 .2 Connectivity ................ 71

4.5 .3 Shear Stress Ratio . . . . . . . . . . . . 71

4.5.4 Over-damped Regime: Scaling Flow Curves . . 72

4.5.5 Under-damped Regime: Emergence of Inertial

Flow . . . . . . . . . . . . 73

4.6 Mechanical Instability in Under-damped Flow: Shear

Bands . . . . . . . . . . . . . . . . 75

4.6.1 Stress Bands: Sink of Energy . . . . . . . . 78

4.6.2 Ramping Simulation: Hysteresis in $\sigma(\dot{\gamma}) \ldots$. . 80

4.6.3 Banded vs. Homogeneous Flow: Local Volume

Fraction ................ 81

4.7 Reservoir Dissipation . . . . . . . . . . . . 83

4.7.1 Flow Curves . . . . . . . . . . . . . . . 84

4.7.2 Viscosity: Thinning and Thickening . . . . . 85

5 THERMALLY ACTIVATED SYSTEMS: FLUCTUATIONS IN CONTACTS 89

5.1 Introduction . . . . . . . . . . . . . . 89

5.2 Random Forces: Implementation . . . . . . . . . . 89 
5.2.1 Thermal Stress and Thermal Time Scales . . . . 90

5.3 Flow Curves: From Athermal to Thermal Systems . . . 91

5.3.1 Stress in Attractive Regime vs. $\tau$. . . . . . . . 92

5.3 .2 Potential Energy vs $\tau \ldots \ldots \ldots$. . . . . . . . . 93

5.4 Connectivity . . . . . . . . . . . . . . . 94

5.5 Structure of the System . . . . . . . . . . . . . . . . 98

5.5 .1 Structure Factor . . . . . . . . . . . . . . . . 98

$5 \cdot 5.2$ Intermediate Scattering Function . . . . . . . . . 99

5.6 Particle Dynamics . . . . . . . . . . . . . . . 101

5.7 Phase Separation in the presence of temperature . . . 105

5.7.1 Order Parameter: Number of clustered particles 106

5.7.2 Phase-separation Parameter Space . . . . . . . . 107

5.8 Different Dissipation Models: Preliminary Results . . . 109

III CLOSING WORDS 113

6 CONCLUSION AND OUTLOOK 115

$\begin{array}{ll}\text { BIBLIOGRAPHY } & 117\end{array}$ 


\section{LIST OF FIGURES}

Figure 1.1 Components of the stress tensor $\sigma$ for a unit volume of a material. Surface normal vectors are indicated by red dashed vectors. . . . . . . .

Figure 1.2 A sketch of the deformation of a material in response to the applied shear stress $\sigma_{x y}$. The deformation is measured by the shear strain, $\gamma=\Delta x /$ L. . . . . . . . . . . . . .

Figure 1.3 A fluid in a simple shear geometry (laminar flow). The fluid is confined between two plates. While the lower plate is stationary, the upper one is moving with a constant velocity $v$. . . Figure 1.4 Possible behavior of a fluid in response to an applied stress. The viscosity, $\eta=d \sigma / d \dot{\gamma}$, is constant for newotonian fluids, increasing for shear-thickening flows and decreasing for shearthinning flows. . . . . . . . . . . . .

Figure 1.5 Microstructure and corresponding viscosity of shear-thinning and shear-thickening regimes in hard-sphere colloidal suspensions. The picture is adapted from [Wagner and Brady, 2009], with the permission of the American Institute of Physics. 9

Figure 1.6 Different shear geometries, often used to shear granular materials. (a) plane shear, (b) Couette cell, (c) silo, (d) incliend plane, (e) flows on a plie, (f) rotating drum. Red arrows indicate velocity field in each geometry. This figure is adapted from [Forterre and Pouliquen, 2008]. . 10

Figure 1.7 The jamming phase diagram for a system at zero temperature and repulsive interactions. Below the jamming point, $\phi_{\mathrm{J}}$, the system behaves as a fluid. Above the jamming point, the yield stress line needs to crossed to flow the system.

Figure 1.8 The jamming phase diagram proposed by Liu Andrea J. and Nagel Sidney R., 1998. Outside the shaded area, where the temperature is high or the applied stress is larger than the yield stress, or the system is dilute enough, the system behaves as a fluid. Please note that the zero-temperature plane is identical to Figure 1.7. This figure is obtained from [Liu Andrea J. and Nagel Sidney R., 1998] with the permission. . . 14 
Figure 1.9 The unified Jamming phase diagram for thermal and athermal systems, obtained by Ikeda, Berthier, and Sollich, 2012. For systems at finite temperature, the jamming transition occurs inside the glass phase, $\phi_{\mathrm{J}}>\phi_{\mathrm{G}}$. This figure is obtained from [Ikeda, Berthier, and Sollich, 2012] with the permission. . . . . . . . . 15

Figure 1.10 Phase diagram for frictional systems. Reentrant flows exist at small and large stress. The figure is adapted from [Grob, Heussinger, and Zippelius, 2014], with the permission. . . . . . . .

Figure 1.11 Velocitiy fields of a shear banded flow in planer shear geometry and corresponding flow curves. (a) Banded flow with finite values of $\dot{\gamma}_{h}$ and $\dot{\gamma}_{l}$ and (b) a yield stress fluid in a shear-banded state. For yield stress fluids, $\dot{\gamma}_{l}=0$ and the system is shear banded for all $\dot{\gamma}<\dot{\gamma}_{h}$. . . . . .

Figure 2.1 Lees-Edwards boundary condition for a system in simple shear flow. The system, represented as a green cell in middle, has normal periodic boundaries is the direction of flow. However, upper and lower periodic images of the system are moving in opposite directions, with constant speeds $\pm \dot{\gamma} \mathrm{L}$, with $\dot{\gamma}$ denoting the applied strain rate and $\mathrm{L}$, being the length of the system in flow gradient direction. . . . . 2 21

Figure 3.1 Interparticle potential $V(r)$, for $\epsilon=1$ and $u=$ 0.05. $\mathrm{d}$ is the summation of particles radii. The inset shows the corresponding elastic force. The attractive part is shown green. . . . . . . . 28

Figure 3.2 (Left), The jamming phase diagram for repulsive systems in the zero temperature plane. (Right), Typical flow curves for a repulsive system in both sides of the jamming point. . . . . . . . 29

Figure 3.3 Flow Curves, $\sigma=\sigma(\dot{\gamma})$ for (a) different attraction range, $u$, at a fixed volume fraction $\phi=$ 0.82 and (b) for different volume fractions, $\phi$, at a fixed attraction range, $u=2 \times 10^{-4}$. The dashed line corresponds to the repulsive system, $u=0.0$. For such systems, the jamming transition occurs around $\phi_{\mathrm{J}} \approx 0.8430 . \ldots .31$ 
Figure 3.4 Two snapshots of a dilute system with $\mathrm{N}=$ $1000, u=2 \times 10^{-4}$ and $\phi=0.40$. (Left), All particles form a large cluster and flow due to shearing. (Right), Later in the same system, particles form two isolated clusters which do not interact for a long time. Arrows indicate the shearing direction. . . . . . . . . .

Figure 3.5 A typical $(p, v)$ diagram of the van der Waals gas with the reduced equation of state as $(p+$ $\left.\frac{3}{v^{2}}\right)(3 v-1)=8 \mathrm{t}$, at the constant temperature $t=0.8$. The red line indicates the non-monotonic part, where $\partial \mathrm{p} / \partial v>0$ and the system is not stable. The dashed line is the phase coexistence line, obtained from the Maxwell equal area construction, replacing the two shaded regions with equal area. Along that line, two phases of the system, gas and liquid, coexists with the same pressure. . . . . . . . . . . .

Figure 3.6 (Left), The jamming phase diagram for weakly attractive systems: The black line indicates $\sigma_{y}(\phi)$ for $u=2 \times 10^{-4}$. Above the Jamming point, yield stress behaves as $\sigma_{y} \propto \delta \phi^{\alpha}$ where we found $\alpha=1.04$. Below the jamming point $\phi_{\mathrm{J}} \approx$ 0.8430 , finite $\sigma_{y}$ and also non-monotonic flow curves are observed. (Right), The phase space of $u$ and $\phi$, where points to observe the nonmonotonic (cross symbols) or monotonic (plus symbols) flow curves are determined. The threshold $\phi$ of the transition between non-monotonic and monotonic flow curve decreases with the attraction strength. . . . . . . . . . .

Figure 3.7 Different kind of connectivity as a function of strain rate for a system at $\phi=0.75$ and $u=$ $2 \times 10^{-4}$. At small strain rates, attractive contacts play important role and keep the total connectivity larger than the isostatic value. at large strain rates, repulsive contacts are dominant, determining the total connectivity behavior. The drop in $z(\dot{\gamma})$ from isostatic point to the value much smaller, indicates the attractive timescale $\tau_{a} \ldots \ldots \ldots \ldots \ldots$

Figure $3.8 \quad z(\dot{\gamma})$ for different volume fractions. The dashed line corresponds to the repulsive system $u=$ 0.0 , solid lines represent attractive system with $u=2 \times 10^{-4} \ldots \ldots \ldots \ldots \ldots$ 
Figure 3.9 The distance to the yield connectivity $\delta z=z_{y}-$ $z$, versus rescaled strain rate $\dot{\gamma} \tau_{a}$. Rescaling the strain rate with attractive time-scale, collapse all $\delta z$ obtained from different values of $u$ and $\phi<\phi_{\text {J }}$ on a single curve. While different colors indicated different $u$, symbols represents different $\phi . \ldots \ldots \ldots \ldots$. . . . . . . . 38

Figure 3.10 Figure 3.11

Figure 3.12

Figure 3.13

Figure 3.14

Figure 3.15

Figure 3.16

Figure 3.17
Sketch of a pair of particles at the yielding point. 39 Yield stress $\sigma_{y}$ normalized by $|\delta \phi|^{\alpha}$ as a function of the combination $u^{1 / 2} \delta z^{3 / 2}$. For $\phi<\phi_{j}$, Equation 3.16 holds. For $\phi>\phi_{j}$ (Dashed line), yield stress shows the expected repulsive behavior $\sigma_{y} \sim|\delta \phi|^{\alpha}$ (Dotted line). . . . . . . . . Potential Energy E as function of strain rate $\dot{\gamma}$ for different volume fractions. The dashed line represents repulsive system with vanishing energy at the limit of zero strain rate. Solid lines are associated with attractive systems with the attraction range $u=2 \times 10^{-4} \ldots \ldots \ldots 4_{4}$ Yield potential energy $E_{y}$ as a function of $\phi$ for different attractions $u$ in (a) repulsion-dominated regime, $\phi>\phi_{\mathrm{J}}$ and (b) attraction-dominated regime, $\phi<\phi_{\mathrm{J}}$. (c) Scaled $E_{y}(\phi)$ by $u^{2}$ in the attraction-dominated regime. . . . . . . . 41 Shear-stress ratio as a function of $\dot{\gamma}$ for different attraction ranges at $\phi=0.75$ (left panel) and for different volume fractions at $u=2 \times$ $10^{-4}$. Note that for $\phi<\phi_{\mathrm{J}}$, the maximum peak appears close to $\dot{\gamma}^{*}$ where $\sigma\left(\dot{\gamma}^{*}\right)$ is minimum. . For a system at $\phi=0.75, \mu_{y}$ (blue curve) and the height of the maximum peak in $\mu(\dot{\gamma})$ (green curve), are plotted as functions of attraction strength, u. . . . . . . . . . . . . . . Pressure $\mathrm{P}$ as a function of strain rate $\dot{\gamma}$ for different volume fractions. The attraction range is $u=2 \times 10^{-4}$. The discontinuity in $\mathrm{P}(\dot{\gamma})$ at $\phi=0.65$ corresponds to negative values of $P$ (compressive pressure). . . . . . . . . . . Structure factor $S(q)$ for different $\dot{\gamma}$ at $u=2 \times$ $10^{-4}$ and $\phi=0.75$. There is a small peak $S^{*}$ for $\mathrm{q}<2$ associated with the local structure and particle clusters in the attraction-dominated regime. Since data points were noisy according to the small system size, they have been smoothed using the Savitzky-Golay filter. . . . . . . . . . 45 
Figure 3.18 Two snapshots of the system in the steady state at $\phi=0.75$ and $u=2 \times 10^{-4}:(\mathbf{a})$, in the attraction-dominated regime, where $\dot{\gamma}=1 e-6$ and $(\mathbf{b})$, in the repulsion-dominated regime, where $\dot{\gamma}=1 e-3$. While the local structure and small clusters of particles, as well as voids, appear in the attraction-dominated regime, the system in the repulsion-dominated regime is homogeneous. . . . . . . . . . . .

Figure 3.19 The first maximum in the structure factor, $\mathrm{S}^{*}$ as a function of $\dot{\gamma} \tau_{\mathrm{a}}$ for a system at $\phi=0.75$ and different attraction strength. The attractive timescale $\tau_{a}$ is used to rescale the strain rate $\dot{\gamma}$. Figure 3.20 Partial structure factor $\mathrm{S}(\mathrm{q})$ for a system at $\mathrm{u}=$ $2 \times 10^{-4}, \phi=0.75$ and $\dot{\gamma}=1 e-6 . S_{00}$ corresponds to the smaller particles, while $S_{11}(q)$ measures the structure factor of larger particles. 47

Figure 3.21 Displacement fields for a system at $u=2 \times$ $10^{-4}, \phi=0.75$ and different $\dot{\gamma}$. In upper panels, displacements are measured over strain window $\Delta \gamma=10^{-2}$, while in lower panels the strain window is $\Delta \gamma=1 \ldots \ldots \ldots \ldots$

Figure 3.22 non-affine mean-squared displacement for (a) where the system is close to yield regime, (b) and (c) around the minimum in the flow curve where the attractive and repulsive branches meet and (d) in the repulsion-dominated regime for a system at $u=2 \times 10^{-5}$ and $\phi=0.75$. . . .

Figure 3.23 Diffusion constant in gradient direction, $\mathrm{D}_{y}$ as a function of rescaled strain rate, $\dot{\gamma} \tau_{a}$, for a system at $\phi=2 e-5$ and different $u . \ldots . .$.

Figure 3.24 Probability distributions of non-affine velocity in the shearing direction, for a system at $u=$ $2 \times 10^{-4}, \phi=0.75$ and different $\dot{\gamma} . \ldots \ldots$

Figure 3.25 Variation of non-affine mean-squared velocity and diffusion constant with strain rate (scaled by the attractive time-scale) in shearing and gradient directions for a system at $\phi=0.75$. While solid lines represent the shearing direction, dashed lines correspond to the gradient direction. . . . . . . . . . . . . .

Figure 3.26 Non-monotonic flow-curves for systems at (left) $\phi=0.82$ but different attraction and (right) $u=2 \times 10^{-4}$ but different volume fractions. Dashed lines indicates the fitted function derived from the constitutive equation 3.38 . . . . 
Figure 3.27 the stress contribution of $\sigma=\eta(\dot{\gamma}) \dot{\gamma}$ as a function of strain rate $\dot{\gamma}$, for a simple model described in Equation 3.44. To obtain $\eta(\dot{\gamma})$, we set $\eta_{0}=200, \eta_{\infty}=0.1$ and $\alpha=500 . \ldots \ldots$

Figure $3.28 \quad$ (a) The velocity profile of a shear banded system. (b) The strain rate profile of the same system as (a). (c) the variation of $\sigma=\eta(\dot{\gamma}) \dot{\gamma}$ for the same system as (a). (d), the second derivative of the local strain rate, $d^{2} \dot{\gamma}(y) / d y^{2}$ as a function of $y$ for the same system as (a). . . . .

Figure 3.29 A snapshot of the non-affine velocity field in a system exhibiting shear-banding, using $\mathrm{N}=$ $2 \times 10^{4}$ particles, $\phi=0.82, u=2 \times 10^{-5}$ and $\dot{\gamma}=2.5 \times 10^{-6}$. The coloring is based on the velocity in the shearing direction. Stream lines show the non-affine flow field. The corresponding connectivity profile reveals that in the solid band, connectivity fluctuates around the isostactic value but decreases in the fludized band. .

Figure $3 \cdot 30$ Flow curves for different system sizes $N$, at $\phi=0.82$ and $u=2 \times 10^{-5}$. The non-monotonic part of the flow curve gets smaller as system size increases and the system is sheared longer. Filled symbols indicate shear band formation.

Figure 3.31 Position of the fludized band in time as the imposed $\dot{\gamma}$ is ramped up. Larger fluctuations at smaller $\dot{\gamma}$ is consistent with the results for the non-affine motion of particles in small systems. 61

Figure 3.32 Spatial profiles of local strain rates, normalized by $\dot{\gamma}$, as it is ramped up. The contrast in the flow rates of bands decreases with increasing $\dot{\gamma}$. 61

Figure 3.33 Shear bands and the interface width in units of system size, as a function of imposed strain rate $\dot{\gamma}$. The dashed line is the fitted linear function to the size of fluid band, $S_{\mathrm{f}}(\dot{\gamma})=4.31 \times$ $10^{4} \times \dot{\gamma}+6.51 \times 10^{-2}$. The dotted line presents the fitted function to the size of solid band which is by definition $S_{s}(\dot{\gamma})=1-S_{f}(\dot{\gamma}) \ldots \ldots 62$

Figure 4.1 Time evolution of a damped harmonic oscillator, in three different damping regimes. Data point are calculated from Equation 4.6, with $A_{1}=1, A_{2}=0$ and $\omega_{0}=1$. Damping factor $\beta$ is set to $\beta=2$ for over-damped, $\beta=0.15$ for under-damped and $\beta=1.0$ for critical damping. 64

Figure 4.2 Flow-curves for a system with $N=1000$, at $u=7 \times 10^{-4}$ and $\phi=0.75 . \ldots \ldots 67$ 
Figure 4.3 Same flow-curves as Figure 4.2, where the damping time-scale, $\tau_{d} \sim 1 / \mathrm{b}$ is used to rescale $\dot{\gamma}$. Data points in the attraction-dominated regime with different $b$, collapse on a single curve. . .

Figure 4.4

Figure 4.5 The potential energy $E_{\text {pot }}$ as a function of damping factor $b$ in the $C_{n}$ model: (left) in the repulsion-dominated regime with $\dot{\gamma}=5 \times 10^{-3}$ and (right), in the attraction-dominated regime with $\dot{\gamma}=10^{-6} \ldots \ldots \ldots \ldots \ldots$ Connectivity for attractive systems $(u=7 \times$ $\left.10^{-4}, \phi=0.75\right)$ with $\mathrm{CD}_{\mathrm{n}}$ dissipation model, at different damping b. (Left), connectivity as a function of strain rate. (right), $\delta z$, distance to the yield connectivity, as a function of rescaled strain rate $\dot{\gamma} \mathrm{b}^{-1 / 4} \ldots \ldots \ldots \ldots$

Figure 4.6 Flow-curves for a system using CD model with $\mathrm{N}=1000, \mathrm{u}=2 \times 10^{-5}, \phi=0.75$ and different damping coefficient $b$. The dashed curve represents the flow-curve of the same system but using the $\mathrm{CD}_{\mathrm{n}}$ model with $\mathrm{b}=2 \ldots \ldots \ldots$

Figure 4.7 Connectivity as a function of strain rate for a system at $u=2 \times 10^{-5}$ and $\phi=0.75$ with different damping factors, $b$ (CD model). . . . Figure 4.8 Shear-stress ratio $\mu$ as a function of strain rate for a system at $u=2 \times 10^{-5}$ and $\phi=0.75$ with different damping factors, $b$ (CD model). The left panel displays the data for over-damped systems and the right panel corresponds to the under-damped regime. . . . . . . . . .

Figure 4.9 Pressure as a function of strain rate for a system at $u=2 \times 10^{-5}$ and $\phi=0.75$ with different damping factors, $\mathrm{b}$ (CD model). $\mathrm{P}(\dot{\gamma})$ increases slower compared to $\sigma(\dot{\gamma})$ at the yieldviscous and viscous-inertial flow transitions. .

Figure 4.10 Weissenberg number, $\mathrm{Wi}=\mathrm{b} \dot{\gamma} / \epsilon u$ is used to rescale (left) flow curves and (right), connectivity of systems at $\phi=0.75$ with different attraction strength $u$ and damping factor $b$ (Overdamped regime). . . . . . . . . . . .

Figure 4.11 Shear-stress ratio $\mu$, for attractive systems at $u=2 \times 10^{-5}, \phi=0.75$, with different damping factors $b$, in the over-damped regime. Weissenberg number is used as a rescaled strain rate, resulting in the collapse of all $\mu$ on a single curve. . . . . . . . . . . 75 
Figure 4.12 Flow curves for a system with $u=2 \times 10^{-5}$ and $b=0.05$ at different volume fractions. The non-monotonic viscous-inertial transition is shifted to smaller $\dot{\gamma}$ as the system becomes more dilute. 76

Figure 4.13 Flow curves for under-damped flows with $\phi=$ 0.75 but different attraction strength $u$ and damping factor $b$. Increasing the attraction strength increases the strain rate at which the non-monotonicity in $\sigma(\dot{\gamma})$ occurs, exhibiting a viscous-inertial flow transition. . . . . . . . . . 76

Figure 4.14 Connectivity $z(\dot{\gamma})$ for under-damped systems with $\phi=0.75$ but different attraction strength $u$ and damping factor $b$. Increasing the attraction strength increases the strain rate at which $z$ drops to the values below 1.0, corresponding to the emergence of inertial flow. . . . . . . . 77

Figure 4.15 Velocity, local strain rate and connectivity profiles of a shear-banded system at $u=2 \times 10^{-5}$, $\phi=0.75, \mathrm{~b}=0.05$ and $\dot{\gamma}_{0}=2 \times 10^{-4}$. The inertial shear band $(z \approx 0.0)$ forms in coexistence with a viscous band where although a percolated structure develops, still $z<z_{\text {iso }}$. . . . . $\quad 78$

Figure 4.16 The stress field and corresponding velocity and connectivity profiles of a shear-banded system at $u=2 \times 10^{-5}, \phi=0.75, b=0.05$ and $\dot{\gamma}=2 \times$ $10^{-4}$. Colored areas determine finite $\sigma$ while in the white area associated with the inertial band, $\sigma$ is orders of magnitude smaller, close to $0.0 \ldots \ldots \ldots \ldots \ldots \ldots$. . . . . . . . . . 79

Figure 4.17 Time averaging of the stress field presented is Figure 4.16 (same color coding), over strain windows of different sizes. The stress field is heterogeneous even after averaging over $\Delta \gamma=$ $2.0 \ldots \ldots \ldots \ldots \ldots \ldots$. . . . . . . . 80

Figure 4.18 Pressure (normal stress) fields for the same system as Figure 4.16. (a) $\log (|\mathrm{P}|)$, pressure (virial) displays a similar discontinue jump at the interface. (b) $\log \left(\left|\mathrm{P}_{\text {tot. }}\right|\right)$, total pressure (including kinetic term) continuously varies at the interface. 80 
Figure 4.19 Flow curves obtained from random initial state at each $\dot{\gamma}$ and also ramping protocol for a system at $u=2 \times 10^{-5}, \phi=0.75$ and $b=0.05$. The red curve indicates the random initial onfiguration. The blue curve presents $\sigma(\dot{\gamma})$ obtained by ramping down $\dot{\gamma}$. At $\dot{\gamma}=3.5 \times 10^{-5}$ the ramping direction is reversed and the obtained $\sigma(\dot{\gamma})$ in displayed by the green curve. Filled symbols indicate strain rates at which shear-banding occurs. . . . . . . . . . .

Figure 4.20 Different profiles of a system at $u=2 \times 10^{-5}$, $\phi=0.75, \mathrm{~b}=0.05$ and $\dot{\gamma}=2 \times 10^{-4}$ in both shear-banded and homogeneous states. (a) displays the normalized velocity profiles, (b) presents the corresponding profile of $\phi$ which is nonuniform in the banded system. The inertial band is much more dilute than the viscous band (c) exhibits the connectivity profile and (d) demonstrates the number of particles as a function of $y$, indicating there are fewer particles in the inertial band compared to the viscous band. . . .

Figure 4.21

Figure 4.22

Figure 4.23

Figure 4.24
Distribution function of $\phi$ for a system at $u=$ $2 \times 10^{-5}$, the initial volume fraction $\phi_{0}=0.75$, $\mathrm{b}=0.05$ and $\dot{\gamma}=2 \times 10^{-4}$ on different ramping branches. For the shear-banded system (rampeddown branch), $\mathrm{P}(\phi)$ is wider with a peak corresponding to the denser $\phi$ with respect to $\mathrm{P}(\phi)$ in the homogeneous system (ramped-up branch). 84 Flow Curve of repulsive systems $(u=0.0)$ at $\phi=0.75$ with $\mathrm{RD}$ and $\mathrm{CD}_{\mathrm{n}}$ dissipation models. (Left), Flow curves obtain with $\zeta=10^{-2}$ and diffrent $b$. (Right), Flow curves obtained with $b=2.0$ and different $\zeta \ldots \ldots . \ldots . . . .85$ Flow curve for attractive systems at $\phi=0.75$ with both $\mathrm{RD}$ and $\mathrm{CD}_{\mathrm{n}}(\mathrm{b}=2)$ dissipation models. (Left), attraction range is set to $\mathrm{u}=$ $2 \times 10^{-5}$, while repulsive flow curves are plotted as dashed curves for comparison. (Right), Attractive flow curves for $u=2 \times 10^{-5}$ (solid lines) and $u=2 \times 10^{-4}$ (dashed lines). In both panels, different colors indicates different $\zeta$. . . viscosity of repulsive systems with different damping factor $\zeta$, as a function of rescaled strain rate, $\dot{\gamma} \tau_{d} \ldots \ldots \ldots \ldots \ldots$. . . . . . 87 
Figure 4.25 Flow curves of attractive systems with RD model, at $u=2 \times 10^{-5}$ and $\phi=0.75$, for different damping factors, $\zeta$. (Right), shear stress as a function of strain rate, (Left), corresponding viscosities. . . . . . . . . . . . .

Figure 5.1 Flow curves for a system with $\mathrm{N}=1000, \mathrm{u}=$ $2 \times 10^{-4}$ at (a) $\phi=0.75$, (b) $\phi=0.82$ and different temperatures $\tau$. Stress is presented in units of thermal stress scale $\left(\sigma_{T}=\epsilon u^{2} \tau / r^{3}{ }_{c}\right)$ and the strain rate is rescaled by the thermal time-scale $\left(t_{T}=r_{c}\left(m / K_{B} T\right)^{1 / 2}\right) . \ldots \ldots$

Figure 5.2 The yield stress $\left(\sigma_{y}\right)$ times the distance to the jamming point $(\delta \phi)$, as a function of $\tau$ for systems at $\phi=0.75$ and $\phi=0.82$. The range of attraction is set to $u=2 \times 10^{-4}$. For $\tau<1$ (attractive regime), $\sigma_{y}$ exhibits an exponential decay with increasing $\tau$ (solid red line). For $\tau>1$ (thermal regime), the shear stress displays a linear increase with $\tau$ (dashed line).

The potential energy at the limit of zero strain

Figure $5 \cdot 3$ rate, $\left(E_{0}\right)$, as a function of $\tau$ for systems at $\phi=$ 0.75 and $\phi=0.82$. The range of attraction is set to $u=2 \times 10^{-4} \ldots \ldots \ldots \ldots$

Figure 5.4 Connectivity $z$ as a function of $\dot{\gamma}$ for an attractive system at different $\tau$ and (a) $\phi=0.75$ (b) $\phi=0.82$. The attraction range is set to $u=2 \times 10^{-4} \ldots \ldots \ldots \ldots \ldots$ Figure $5.5 \quad z(\tau, \dot{\gamma})$ for an attractive system at $u=2 \times 10^{-4}$ and $\phi=0.75$. For such a system below the glass point $\phi_{\mathrm{G}}, z(\tau)$ decreases at the athermalthermal transition. . . . . . . . . . . . . 96

Figure $5.6 \quad z(\tau, \dot{\gamma})$ for an attractive system at $u=2 \times 10^{-4}$ and $\phi=0.82$. Relatively large values of $z$ is observed at fast shearing, regardless of $\tau$. At small $\tau$ shear-induced collisions are responsible for keeping connectivity large, $z \approx 3$ and at large $\tau$, thermal collisions. . . . . . . . . . 96

Figure 5.7 The yield connectivity $z_{y}$ as function of effective temperature, $\tau$, for an attractive system at $u=2 \times 10^{-4}$ and $\phi=0.82$. The total connectivity is plotted by blue solid line, the repulsive connectivity is indicated by the green dashed line and the attractive connectivity is represented by the red dashed line. . . . . . . . 
Figure 5.8 The structure factor $\mathrm{S}(\mathrm{q})$ for a system at $\mathrm{u}=$ $2 \times 10^{-4}, \phi=0.75$ and different $\tau$. The small peak, corresponding to clusters of particles, disappears as $\tau$ moves towards the thermal regime. 98

Figure 5.9 Partial structure factors of a system at $u=2 \times$ $10^{-4}, \phi=0.75$ and $\dot{\gamma}=10^{-6}$. (Left) $S_{00}$ corresponds to the structures consisting of only small particles, (Right) $S_{11}$ measures the structure factor only for large particles. . . . . . . .

Figure 5.10

Figure 5.11

Figure 5.12

Figure 5.13

Figure 5.14
The corresponding length-scales of peaks in the structure factor $S(q)$. $r_{1}$ represents the peak in $S(q)$ when $q<2$, associated with micro-phase separation at small $\tau$. $r_{2}$ corresponds to the largest peak of $S(q)$ at $q \approx 2 \pi . \ldots \ldots . . .100$ The intermediate scattering function $F(q, \gamma)$ for a system at $u=2 \times 10^{-4}, \phi=0.75$ and different $\tau$. We take $q=5.71$ corresponding to the large peak in the $S(q) . \ldots \ldots . . \ldots 100$ non-affine mean-squared displacement for where the system is at (a) $\phi=0.75$ and close to the yield regime, $\dot{\gamma}=10^{-6}$, (b) at $\phi=0.75$ and in the repulsion-dominated regime, $\dot{\gamma}=10^{-3}$, (c) at $\phi=0.82$ above $\phi_{\mathrm{G}}$ and close to the yield regime $\dot{\gamma}=10^{-6}$ and (d) at $\phi=0.82$ and in the fast shearing regime, $\dot{\gamma}=10^{-3}$. The attraction range is set to $u=2 \times 10^{-4}$. The dashed line represent the diffusive behavior, MSD $\propto \gamma^{1}$ while the dotted line indicates the ballistic behavior, MSD $\propto \gamma^{2} \ldots \ldots \ldots$. . . . . . . . . . 102 MSD in small strains (short times), for a system presented in Figure 5.12, panel (c). Different colors indicate different temperatures, all set the system in the thermal regime $(\tau 1.0)$. at small $\gamma$, a transition from a sub-diffusive to diffusive (or weak super-diffusive) regime takes place, hinting a cage dynamics at small $\gamma$ for systems with $\phi>\phi_{\mathrm{G}}$, in the thermal regime. The dotted line represents MSD $\sim \gamma^{0.79}$, while the dashed line corresponds to MSD $\sim \gamma^{1.2} \ldots \quad . \quad 103$ Variation of non-affine mean-squared velocity with strain rate (scaled by the thermal timescale) in shearing direction for a system at $\phi=$ 0.75 and different $\tau$. The attraction range is set to $u=2 \times 10^{-4} \ldots \ldots \ldots \ldots \ldots$. . . . . 104 
Figure 5.15 The diffusion constant, measured along the gradient direction, as a function of scaled shearrate, for different $\tau$. The system is at $u=2 \times$ $10^{-4}$ and $\phi=0.75 . \ldots \ldots . . \ldots 104$

Figure 5.16 Snapshots of the system exhibiting phase separation at $u=2 \times 10^{-4}, \phi=0.75$ and $\tau=$ $2 \times 10^{-1}$ (a) without shearing $\dot{\gamma}=0$ and (b) with a finite strain rate $\dot{\gamma}=10^{-6} \ldots \ldots \ldots 105$

Figure 5.17 Possible structure of phases for a system displaying phase separation. A phase consists of (a) two particle types, (b) just large particles and (c) just small particles. While all these phases are observed in the phase-separated system at $\dot{\gamma}=0$, shearing the system with a finite rate allows only the formation of phases (b) and (c). 106

Figure 5.18 Number of particles in packed clusters as a function of strain $\gamma$. Data is plotted for systems at (left) tau $=2 \times 10^{-1}$ and different $\dot{\gamma}$, (right) $\dot{\gamma}=10^{-6}$ and different $\tau$. The attraction range is set to $u=2 \times 10^{-4}$ and the volume fraction is $\phi=0.75 \ldots \ldots \ldots \ldots \ldots$

Figure 5.19 The amount of phase-separation for an attractive system at $u=2 \times 10^{-4}$ and $\phi=0.75$. The number of particles in separated phases, $\mathrm{N}^{*}$, is plotted at different values of temperature $\tau$ and strain rate $\dot{\gamma} . \ldots . . . . . . . . . .109$

Figure 5.20

Flow curves of systems with $C D$ dissipation model at $u=2 \times 10^{-5}, \phi=0.75$, (a) $b=2.0$ and $(b) b=0.05$. In the under-damped regime, data is represented in terms of rescaled strain rate, Pećlet number, and rescaled stress $\sigma / \sigma_{\mathrm{T}}$.

Figure 5.21 Flow curves for systems with RD dissipation model at $u=2 \times 10^{-5}, \phi=0.75$, (a) $\zeta=2.0$ and $(b) \zeta=5 \times 10^{-3}$. In panel (b), data is represented in terms of rescaled strain rate, Pećlet number, and rescaled stress $\sigma / \sigma_{\mathrm{T}} . \ldots \ldots$ III 

Part I

INTRODUCTORY MATERIAL 



\section{THEORETICAL BACKGROUND}

\section{I.I SOFT MATERIAL}

Soft condensed matter is the term to refer to materials that are sensitive to external forces, such as thermal and mechanical stress, whose magnitude is comparable to thermal fluctuations. As a response to such external drivings, they are deformed, or change the structure. A large category of soft materials is Complex Fluids, referring to materials that are neither simple liquids nor solids with crystalline structure. We are familiar with plenty of these materials in our daily life. Paints that we use for drawing, food products like tomato ketchup or mayonnaise, health products as toothpaste, soaps and shampoos and many materials which are important in industry as fine powders, granular material and polymer melts, they all belong to the class of soft matter. Foams and emulsions, which are dispersions of gas bubbles or liquid droplets in a liquid, are other examples of soft matter. Most importantly, most of biological systems consist of soft matter.

There are a number of common features that motivates people to consider them as a specific class of material. Because of the importance and applications of such materials, they are of intense interest to researchers. Pierre-Gilles de Gennes, (1932-2007), who is often known as the "founding father of soft matter", won the Nobel Prize in physics in 1991 for his works on soft materials.

Interesting behavior arises from soft matter, which are not easily predictable, if possible at all, from properties of its ingredients. Relevant length scales are often intermediate between atomic sizes and macroscopic scales. Soft matters usually self-assemble in structures at mesoscopic sizes, where quantum effects are unimportant. Therefore, coarse-grained models can be used to study such material, without a need to account for all details on atomic scales.

For instance, common features of complex fluids as an example of soft matter, can be caught by some general models or phenomena, regardless of their dramatic differences on atomic level. In the rest of this chapter, we review some of those models, theories and phenomena, which are necessary to establish a theoretical framework for the result section. We specifically focus on crowding effects and flow properties of particulate systems. 


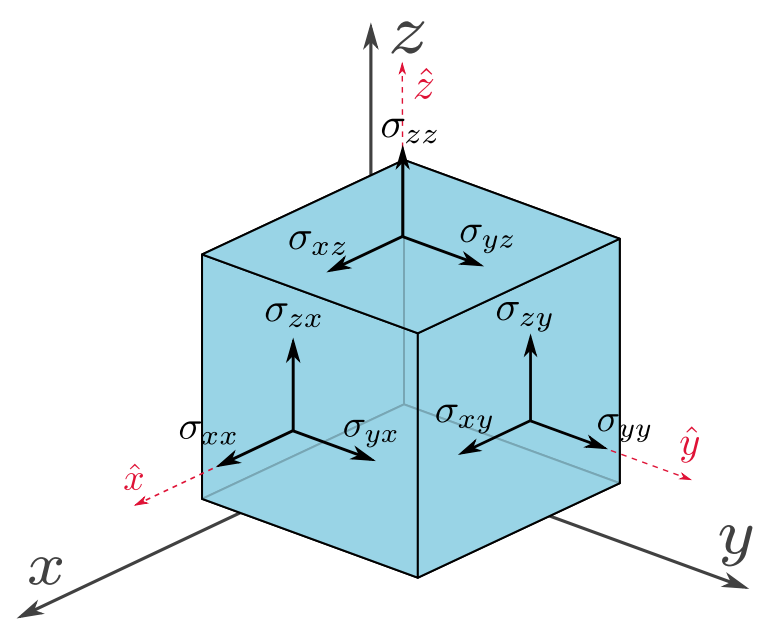

Figure 1.1: Components of the stress tensor $\sigma$ for a unit volume of a material. Surface normal vectors are indicated by red dashed vectors.

Normal condensed matter are known in two general categories, solids and liquids. However, in the case of complex fluids, it is not that simple to classify the system in either of those categories. For example, is the shaving foam, as a typical example of complex fluid, solid or fluid? Without applying a large stress, it seems to behave as a solid and does not flow. But in the presence of an adequate stress, it behaves as a fluid. To understand and explain the complex behavior of soft matter precisely, we first review the definitions of solid and fluid in terms of mechanical response of the system.

\subsubsection{Stress Tensor and Strain}

Consider a volume element of a material presented in Figure 1.I with force $\vec{F}$ exerting on it. Stress $\boldsymbol{\sigma}$ is a second order tensor with nine components measuring the force per area exerted on each face of the volume unit. $\sigma$ components are defined as

$$
\sigma_{i j}=\frac{F_{i}}{A_{j}},
$$

where indexes indicate components in orthogonal Cartesian coordination system. In Equation 1.1, $F_{i}$ indicates the force in $\hat{i}$ direction exerting on the face of the unit volume with a normal surface vector of $\hat{j}$ and the area of $A_{j}$. Indexes $i, j, k$ can take any value from $(\hat{x}, \hat{y}, \hat{z})$.

In general, $\sigma$ components have two different types: for a given surface, $\sigma_{i i}$ is called the normal stress (compression or tension) which is perpendicular to the surface, and the shear stress $\sigma_{i j}(i \neq j)$ that 


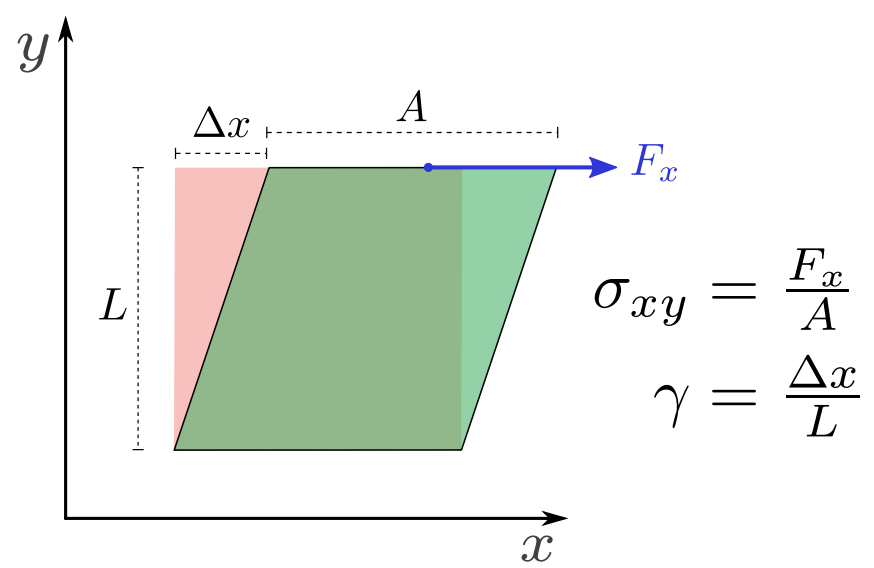

Figure 1.2: A sketch of the deformation of a material in response to the applied shear stress $\sigma_{x y}$. The deformation is measured by the shear strain, $\gamma=\Delta x / \mathrm{L}$.

is parallel to the surface. Please note that the pressure (or the normal stress) of the system is defined as

$$
P=\frac{1}{3}\left(\sigma_{x x}+\sigma_{y y}+\sigma_{z z}\right)
$$

Figure 1.I displays all components of the stress tensor on different faces of the unit volume.

The response of the system to shear stress, $\sigma_{i j}$ in Figure 1.I, can be used to define whether the system is solid or fluid. Solid materials can sustain shear stress while fluids are subjected to flow in response to a shear stress. Figure 1.2 illustrates a two-dimensional sketch of a material deformation due to a shear stress $\sigma_{x y}$. Here, for simplicity we assume $A=A_{y}$, thus by definition, shear stress is defined as $\sigma_{x y}=F_{x} / A$. The deformation can be measured through shear strain (or in short, strain) that is defined as

$$
\gamma=\frac{\Delta x}{\mathrm{~L}}
$$

\subsubsection{Elastic Solid}

For a solid, imposing the shear stress to the material causes a constant strain in response. In the case of simple solids, the strain is proportional to the applied stress as

$$
\sigma_{x y}=\mathrm{G} \gamma
$$

where the factor of proportionality, G, is called the shear modulus. 


\subsubsection{Viscous Fluid}

For a fluid, a constant shear stress leads to a time-dependent strain; the material flows. The rate of changes in strain, is called the strain rate or the shear rate,

$$
\dot{\gamma}=\frac{\mathrm{d} \gamma}{\mathrm{dt}}
$$

where $t$ indicates the time. The strain rate is related to the applied shear stress as

$$
\sigma_{x y}=\eta(\dot{\gamma}) \dot{\gamma}
$$

The factor $\eta$, is called viscosity which in general might be a function of strain rate itself. If we imagine the fluid as several layers of material flowing on top of each others, then $\eta$ can be considered as the resistance of the material to the relative motion of neighboring layers.

It should be mentioned that Equation 1.6 is a tensorial equation as,

$$
\sigma=\eta \nabla \vec{v}
$$

in which $\vec{v}$ represents the velocity vector. In the case of isotropic fluid (no preferred direction for the material), the viscosity tensor reduces to two real components: The shear viscosity, describing the fluid's resistance to the shear deformation and the bulk viscosity, corresponding to the fluid's resistance to compression or expansion.

\subsubsection{Newtonian Fluid}

Newtonian fluids are the simplest mathematical model of fluids at which by definition, the stress $\sigma$, is linearly proportional to the rate of change of the fluid deformation in time, or the strain rate $\dot{\gamma}$,

$$
\sigma=\eta \dot{\gamma}
$$

In such a flow, $\eta$ is constant in time and does not depends on $\dot{\gamma}$.

Figure 1.3 displays a simple shear system or laminar flow, where the fluid is confined between two parallel plates, one fixed and the other moving with a constant velocity, $v$. We assume the top plate is the moving one. If $v$ is small enough, the fluid's particles move parallel to the moving plate and their velocity decreases from $v$ to 0 linearly with the distance to the moving plate. In the larger scale, each layer of the fluid moves faster than the one further from the moving plate. The relative motion of neighboring layers, gives rise to a friction force. In particular, the fluid will exert a force $F$ on the moving plate, resisting its motion. This force should be canceled by an external force to keep the plate moving with a constant speed. If the area of the plates is 


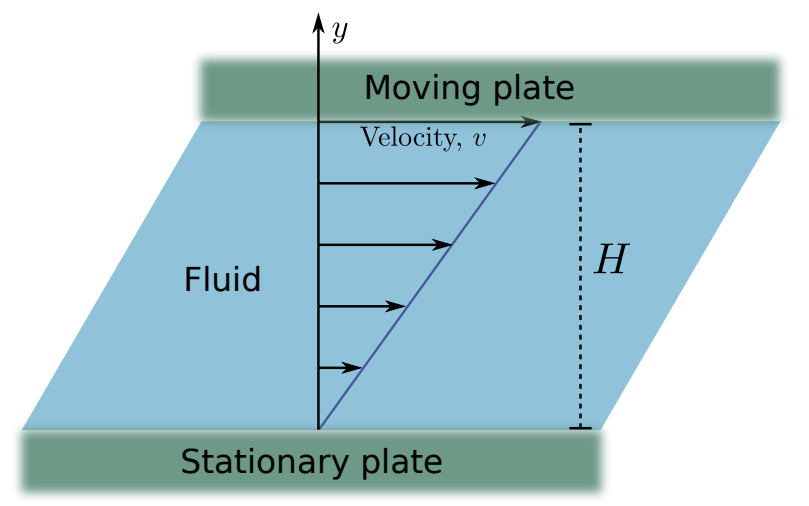

Figure 1.3: A fluid in a simple shear geometry (laminar flow). The fluid is confined between two plates. While the lower plate is stationary, the upper one is moving with a constant velocity $v$.

$A$ and the gap distance between them is $H$, then the force $F$ can be written as:

$$
\mathrm{F}=\eta \mathrm{A} \frac{v}{\mathrm{H}}
$$

where the ratio $v / \mathrm{H}$ is the rate of shear deformation or the strain rate $(\dot{\gamma}=v / H)$ and the viscosity $\eta$ is equivalent to the friction coefficient. In terms of differential equations, shear stress can be written as

$$
\sigma=\frac{F}{A}=\eta \frac{\partial v}{\partial y}
$$

where $\partial v / \partial y$ corresponds to the local strain rate. Equation 1.9 was first derived by Newton, assuming that the flow moves along parallel lines.

\subsubsection{Complex Fluid}

Usually flow behavior of complex fluids are categorized in time-dependent and time-independent behaviors. Time-dependent fluids exhibit a combination of viscous and elastic responses, depending on the time-scale in which the shear stress is applied. This kind of behavior is called viscoelasticity. If a constant stress is applied at time $t=0$, at first the system responds as an elastic solid and deforms with a constant amount of strain, $\gamma=\sigma / \mathrm{G}_{0}$. Here, $\mathrm{G}_{0}$ is the instantaneous shear modulus. However after a certain time $\tau$, it starts to flow as a viscous fluid with a constant strain rate $\dot{\gamma}=\sigma / \eta$. The time $\tau$ is the relaxation time, separating the solid-like behavior from the fluid-like behavior. One famous example of such materials is the silly putty toy, which is made of silicone polymers. It acts as a solid if the stress is applied in a time shorter than the relaxation time. If we throw the toy toward the wall fast enough, it bounces back as an elastic solid. On the other hand, if we just hold the paste at some height, after some time the gravity 
results in the flow of it toward the ground; the paste flows as a viscous fluid. For a Newtonian fluid, one can estimate $\eta$ by balancing the elastic and viscous responses of the material at the relaxation time.

$$
\eta \sim G_{0} \tau
$$

One example of time-independent fluid behavior is the Viscoplasticity, in which stress scales paly the main role to detemine the response of the system. For such materials, a stress threshold, yield stress $\sigma_{y}$, exists which must be exceeded to deform or flow the material. If the externally applied stress is less than $\sigma_{y}$, the material responds as an elastic solid. As it was mentioned earlier, shaving cream exhibits such yield behavior.

Unlike Newtonian fluids, complex fluids usually do not conform to the Newtonian postulate, the linear relation between $\sigma$ and $\dot{\gamma}$, expressed in Equation 1.6. Hence these fluids are also called nonNewtonian Fluids, including foams, dispersions and suspensions, emulsions and polymeric melts, for instance.

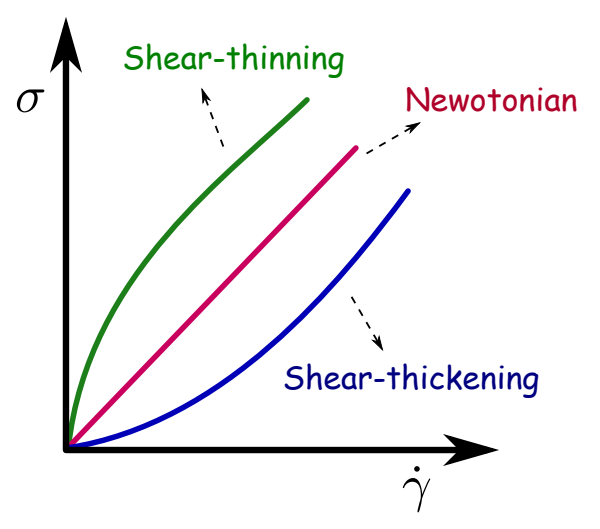

Figure 1.4: Possible behavior of a fluid in response to an applied stress. The viscosity, $\eta=d \sigma / d \dot{\gamma}$, is constant for newotonian fluids, increasing for shear-thickening flows and decreasing for shear-thinning flows.

The main feature of Complex fluids is that the viscosity, $\eta$, may be a varying function of $\dot{\gamma}$ or $\sigma$. Under some circumstances, the viscosity can be even a function of time, depending on the history of the fluid. In this sense, there are three main categories for the response of a fluid to an applied stress. For Newotonian fluids, the viscosity $\eta$ is constant and the strain rate $\dot{\gamma}$ is linearly proportional to the shear stress, as it was discusses above. For some materials, viscosity decreases as the strain rate is increased, called shear-thinning behavior. An example of such materials is paint, which explains why it moves easier as one does faster brushing. Another type of materials are those whose viscosity is an increasing function of strain rate; it gets harder to flow the material in higher rates. This behavior is called shear-thickening 
and is often seen in pastes. Figure 1.4 demonstrates these three types of flow in terms of the stress as a function of strain rate.

Some material as hard sphere colloidal suspensions can experience shear-thinning and shear-thickening behavior at different regimes of applied stress or strain rate [Chen et al., 2010; Wagner and Brady, 2009]. Figure 1.5 displays the connection between the microstructure of the material and the corresponding shear regime. For such systems, the suspension in equilibrium resists to flow as a Newtonian fluid with a constant viscosity. Increasing shear stress (strain rate), organizes particles in a way that the viscosity decreases; shear-thinning occurs. Further increase of the shear stress (strain rate) results in the formation of clusters, due to enhancement of hydrodynamic interactions between particles. Thus, particles face more difficulty to flow, the viscosity increases again and transition to the shear-thickening regime happens [Wagner and Brady, 2009].

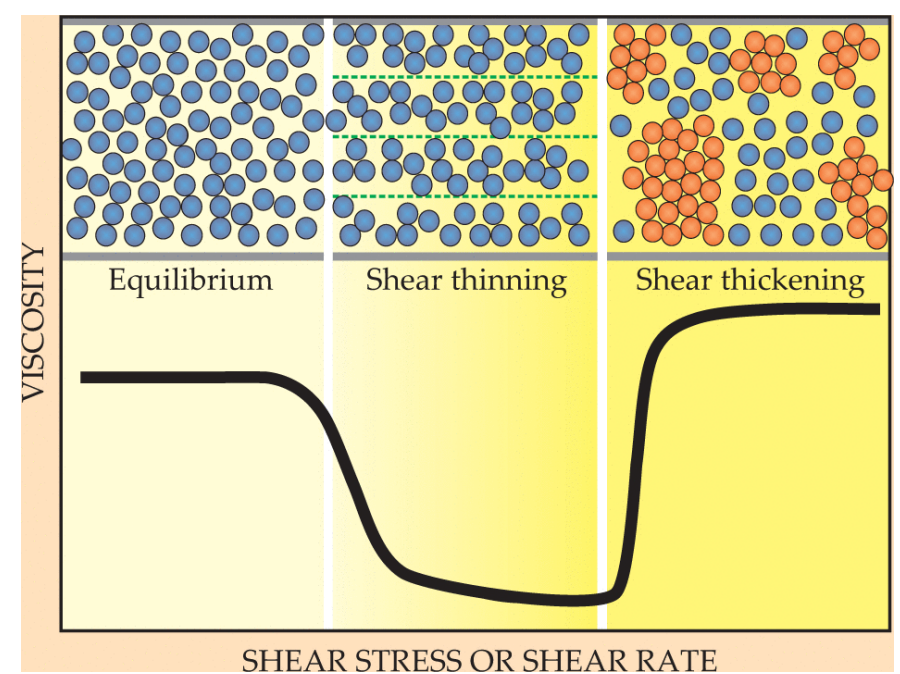

Figure 1.5: Microstructure and corresponding viscosity of shear-thinning and shear-thickening regimes in hard-sphere colloidal suspensions. The picture is adapted from [Wagner and Brady, 2009], with the permission of the American Institute of Physics.

\subsection{RHEOLOGY: STUDY OF FLOW AND DEFORMATION}

Rheology is defined as the study of the flow of materials. It includes flows in a liquid state, as well as behavior of solids which flow under application of external force, instead of exhibiting elastic deformation. It usually deals with materials which have a complex microstructure, such as muds, suspensions, polymers, granular material, as well as bodily fluids (e.g., blood) and other biological materials which are all categorized as soft material.

To make such materials flow, they are often sheared in nature and industry. The shear is sometimes induced by the relative motion of 
boundaries, leading to a relative velocity between them and the bulk material. It is worthy to describe different shear geometries which are common in studies of rheology of soft matter.

\subsubsection{Shear Geometries}

Figure 1.6, adapted from [Forterre and Pouliquen, 2008], displays different geometries used to study rheology of dense granular matter.

a
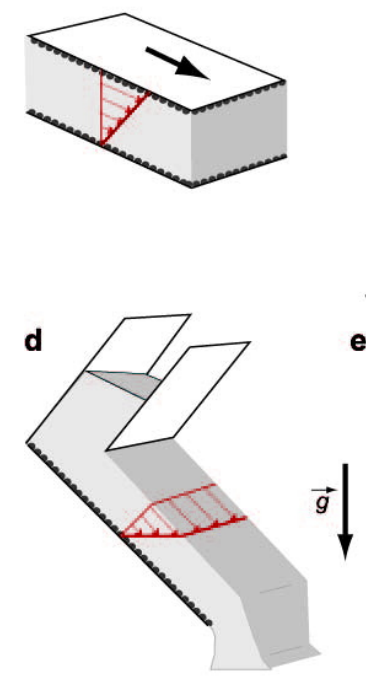

b
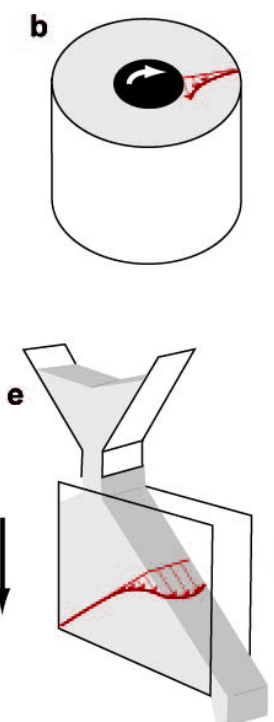

c
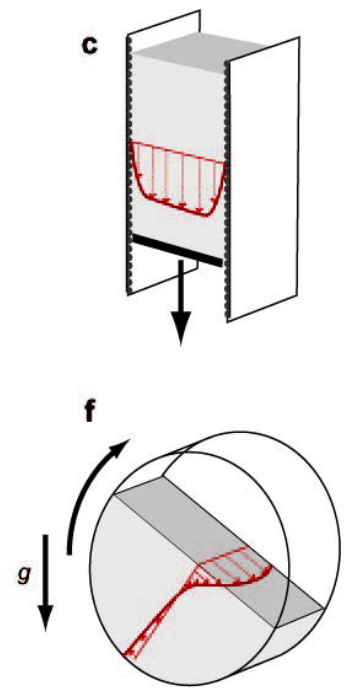

Figure 1.6: Different shear geometries, often used to shear granular materials. (a) plane shear, (b) Couette cell, (c) silo, (d) incliend plane, (e) flows on a plie, (f) rotating drum. Red arrows indicate velocity field in each geometry. This figure is adapted from [Forterre and Pouliquen, 2008].

Among setups depicted in Figure 1.6, planar shear and Couette cell are more common in experiments. Plane shear is the simplest protocol to study the rheology of systems, specially from the computational point of view. There, there is a relative motion of the two parallel boundaries in opposite directions, imposing a shear in the perpendicular direction, the Shearing direction. The distance between upper and lower boundaries can be fixed or flexible, depending on the experiment. In numerical studies, other boundaries stay stationary, with the periodic boundary conditions.

Couette cell is another shear setup which is proper for experiments because of its periodicity in the flow direction. The shear is imposed by the relative rotation of inner and outer cylinders. In another version of Couette cell, the bottom plate can also be rotating with the cylinders. The top boundary, similar to the plane shear, might be fixed or flexible depending on the experiment. Because of the symmetry of Couette cell, one can rotate cylinders as long as it is desired. Hence, large amount of strain would be accessible in laboratories. 


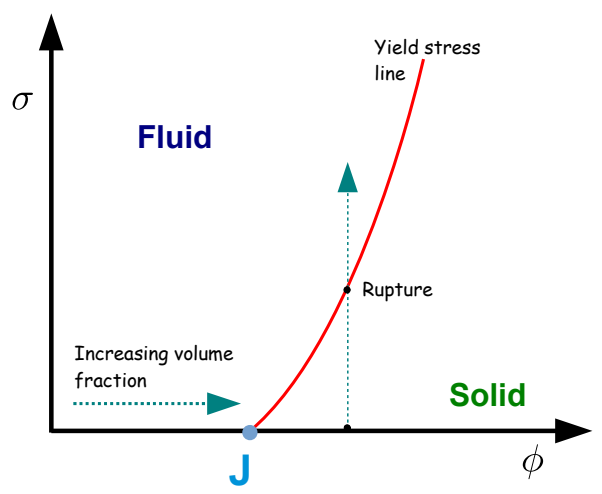

Figure 1.7: The jamming phase diagram for a system at zero temperature and repulsive interactions. Below the jamming point, $\phi_{\mathrm{J}}$, the system behaves as a fluid. Above the jamming point, the yield stress line needs to crossed to flow the system.

In this work, we only focus on the plane shear whose numerical implementation is explained in Chapter 2.

\subsection{THE JAMMING TRANSITION}

Consider a box of volume $\mathrm{V}$ with $\mathrm{N}$ frictionless particles inside it, at zero tempretaure and zero shear stress. Particles interact via repulsive pair potentials that disappears at the cutoff distance, namely the summation of their radii. For such a system, the volume fraction $\phi$ is defined as the fraction of the box volume occupied with particles. For the case of spherical particles, one can write

$$
\phi=\frac{3 \pi}{4 V} \sum_{i=1}^{N} R_{i}^{3},
$$

with $R_{i}$ being the radius of $i$ th particle. For dilute systems (small $\phi$ ), the system acts as a fluid. Any arbitrary stress would be enough to flow the system. Void space in the system helps particles to move easily due to the applied stress. However, increasing $\phi$ gradually reduces the void space between particles. At a specific volume fraction, the jamming point $\phi_{\mathrm{J}}$, particles get jammed and the system starts to behave as a disordered solid. This fluid-solid like transition is called the jamming transition.

For $\phi>\phi_{\mathrm{J}}$, the applied stress needs to exceed a threshold to break the structure of particles and flow the system. That threshold stress is called the yield stress, usually indicated by $\sigma_{y}$. Figure 1.8 represents a so-called jamming phase diagram at zero temperature, adapted from [Liu Andrea J. and Nagel Sidney R., 1998].

The order parameter that characterized the jamming transition is connectivity, which is the average number of contacts per particle, resembled by $z$. For systems below $\phi_{\mathrm{J}}, z=0$ since repulsive interactions 
push away overlapping particles, leading to the disappearance of contacts. However for $\phi>\phi_{\mathrm{J}}$, particles are forced to make contacts due to lack of void spaces. Thereby, a system spanning force (contact) network form, resulting a finite $z$ for a disordered solid. At the jamming transition $\phi=\phi_{\mathrm{J}}, z$ jump discontinuously from $z=0$ to the isostatic connectivity $z_{\text {iso }}$. For frictionless particles, $z_{\text {iso }}$ is the minimum connectivity needed for the emergence of rigidity and mechanical stability in the system. In the d-dimensional system consisting of $\mathrm{N}$ particles, there are $\mathrm{Nd}$ equations describing forces exerting on particles. In order to have mechanical stability, these equations need to be satisfied by $\mathrm{N} z / 2$ inter-particle forces. Therefore, one can conclude $z_{\text {iso }}=2 \mathrm{~d}$ [Maxwell, 1864].

Further compressing of a system to obtain $\phi>\phi_{\mathrm{J}}$, imposes more contacts between particles resulting in larger connectivity, $z>z_{\text {iso. }}$. It is found in numerical simulations that the excess number of contacts grows as [Durian, 1995; O'Hern et al., 2002; O'Hern et al., 2003]

$$
\delta z \propto \delta \phi^{\beta \approx 1 / 2},
$$

with $\delta z=z-z_{\text {iso }}$ is the distance to the isostatic connectivity and $\delta \phi=\phi-\phi_{\mathrm{J}}$ indicates the amount of compression above the jamming point.

\subsubsection{The Value of $\phi_{\mathrm{J}}$}

As we discussed above, the jamming point is attributed to an isostatic arrangement of particles. However, the isostatic structure can be sensitive to the preparation procedure. Each mechanically stable arrangement of particles indicates a local minimum of the potential energy of the system. Imagine a given local minimum of potential energy (E) corresponding to a mechanical stable state of the system. If we decompress the system gradually, the potential energy eventually reaches $E=0$, the resulting volume fraction is the jamming volume fraction $\phi_{\mathrm{J}}$ and the network of particles becomes isostatic.

In this procedure, different initial points may lead to different values of $\phi_{\mathrm{J}}$. Therefore, it should be mentioned that for a given set of particles, there is a distribution of values of $\phi_{j}$ with a vanishing width in the infinite system-size limit [O'Hern et al., 2002; 2003]. So almost all initial points reach the same jamming volume fraction. For mono-disperse packings of spherical particles in three dimensions, it is found that $\phi_{\mathrm{J}} \approx 0.64$, close to the random close packing density. For two-dimensional sets of bi-disperse disks, which is the main interest of this work, the jamming volume fraction is found to be $\phi_{\mathrm{J}} \approx 0.8430$.

However, Chaudhuri, Berthier, and Sastry, 2010, showed that even in the thermodynamic limit, different protocols might lead to different values of $\phi_{\mathrm{J}}$, which are all sharply defined. Therefore, instead of one unique jamming volume fraction, there is finite range of val- 
ues of $\phi_{\mathrm{J}}$, corresponding to the presence of many local minima or metastable states in the energy landscape of the system [Chaudhuri et al., 2010].

\subsection{EFFECT OF TEMPERATURE}

\subsubsection{The Glass Transition}

The emergence of solidity in disordered assemblies of particles is observed in many situations in everyday life, industry and nature. Foams and granular matters are typical examples of materials experiencing the jamming transition, while neglecting the temperature is an appropriate assumption [Liu and Nagel, 2010]. For those materials, even small rearrangements of particles cost the energy orders of magnitude greater than the thermal energy at room temperature.

However, thermal forces are important in the case of relatively small-sized particles. Consider a dilute colloidal suspension, whose properties are determined by the competition between Brownian, hydrodynamic and inter-particle forces. Compressing such a system, it undergoes a glass transition from thermal equilibrium, while its dynamics turns sluggish and the shear viscosity increases dramatically. Such a behavior is observed for a wide range of materials including emulsions, polymer networks, liquid crystals and etc. [Chen et al., 2010]. The volume fraction at which the glass transition takes place is indicated by $\phi_{\mathrm{G}}$.

\subsubsection{Jamming Transition versus Glass Tansition}

For a long time, the jamming transition and the glass transition had been assumed to share physical roots. Both phenomena deal with the emergence of rigidity by increasing the volume fraction of a disordered set of particles, near a critical threshold. The resulting amorphous solid responds elastically to small applied stress, but flows if it exceeds a threshold, the yield stress. This similarity is the main idea behind the jamming phase diagram proposed by Liu and Nagel (see Figure 1.8), where the jamming point $\phi_{\mathrm{J}}$ is assumed to be the limiting value of $\phi_{\mathrm{G}}(\mathrm{T})$ while the temperature $T$ goes to zero [Liu Andrea J. and Nagel Sidney R., 1998].

However, detailed studies in the last few years suggest that the jamming transition takes place well inside the glass phase, indicating that separate microscopic mechanisms are responsible for them [Ikeda, Berthier, and Sollich, 2012; Mari, Krzakala, and Kurchan, 2009]. Numerical studies of the rheology of soft particles reveal that the shear stress contributions from jamming and glass transitions are in fact additive. While the dynamics of colloidal hard spheres is mainly determined by glass physics, materials like foams are more strongly in- 


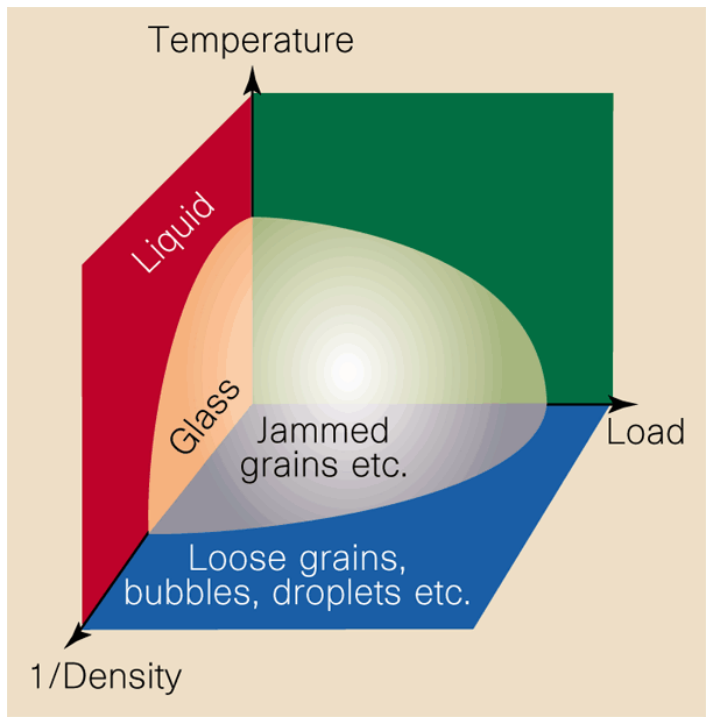

Figure 1.8: The jamming phase diagram proposed by Liu Andrea J. and Nagel Sidney R., 1998. Outside the shaded area, where the temperature is high or the applied stress is larger than the yield stress, or the system is dilute enough, the system behaves as a fluid. Please note that the zero-temperature plane is identical to Figure 1.7. This figure is obtained from [Liu Andrea J. and Nagel Sidney R., 1998] with the permission.

fluenced by the jamming transition. For materials with intermediate particle size like emulsions, both jamming and glass effects are important to account for the flow behavior of the system [Ikeda, Berthier, and Sollich, 2013]. Figure 1.9 displays the phase diagram proposed by Ikeda et al., 2012, including both glass and jamming phases.

In Chapter 5, we study the role of thermal fluctuations on rheology of weakly attractive systems. We explore the crossover from athermal to thermal limit, where glassy dynamics is dominant. The intermediate regime, where thermal fluctuations compete with attraction is of main interest, which will be discussed intensively.

\subsection{ROLE OF PARTICLES INTERACTIONS}

Other than macroscopic parameters like the volume fraction or temperature, microscopic details as particle interactions would also affect the properties of the system. Specially, when the behavior of the system under steady shear is sought, details of particle interactions play a significant role as it is explained by some examples below.

\subsubsection{Dissipation and Inertia}

In order to model an externally driven friction-less system in the athermal limit, e.g. shearing systems, a dissipation mechanism should 


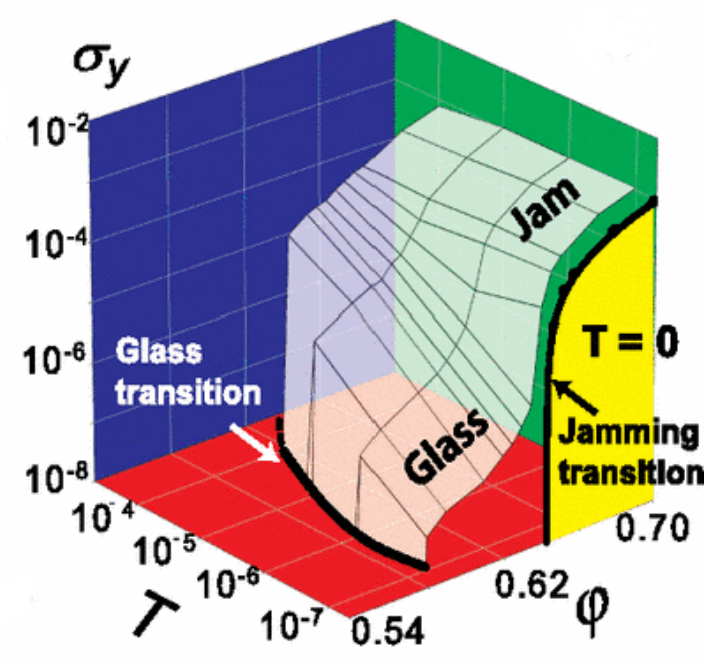

Figure 1.9: The unified Jamming phase diagram for thermal and athermal systems, obtained by Ikeda, Berthier, and Sollich, 2012. For systems at finite temperature, the jamming transition occurs inside the glass phase, $\phi_{\mathrm{J}}>\phi_{\mathrm{G}}$. This figure is obtained from [Ikeda, Berthier, and Sollich, 2012] with the permission.

be considered, otherwise particles heat up. For instance, one can consider foams and emulsion. Microscopically, such materials consist of deformable particles stabilized by surfactants, without experiencing Coloumb-like friction. For bubbles, drag force is governed by means of complex physical mechanisms, including dynamics and interaction between surfactants [Denkov et al., 2008; Höhler and Cohen-Addad, 2005].

From theoretical point of view, the choice of the dissipation and its strength may considerably vary the flow properties of the system. Below the jamming, granular particles flow in a manner that the pressure and the shear stress are proportional to the squared of the strain rate, $\sigma \propto \dot{\gamma}^{2}$ (at low $\dot{\gamma}$ ), which is called Bagnold scaling [Bagnold, 1954]. However, some other materials like foams or emulsions demonstrate the Newtonian fluid behavior, the linear dependence of shear stress on the strain rate $\sigma \propto \dot{\gamma}$ [Boyer, Guazzelli, and Pouliquen, 2011; Durian, 1995]. Other than the mass of particles, the possibility of the formation of large connected clusters of particles is found to be a key parameter to determine whether a system has a Bagnoldian or Newtonian rheology [Vågberg, Olsson, and Teitel, 2014]. In the context of frictionless particles, the Bagnoldian rheology is accompanied by the vanishing connectivity at the limit of zero strain rate, even at dense regime. However, the Newtonian flow behavior is associated with the presence of large connected clusters, formation of which, can be controlled by the dissipation mechanism in particle collisions. If the relative velocity of colliding particles decays to zero during the collision, they remain in contact and eventually, large clusters form. Vågberg 
et al., 2014, showed that the tangential dissipation has a crucial role on the development of such clusters.

Other than the type of the dissipation, its strength is also important to determine the rheology of system. Large damping sets the system in the over-damped regime, where kinetic terms are negligible in particles dynamics. On the other hand, inertia becomes important when damping is small, e.g. when the relative velocity of colliding particles remains finite after the collision. For athermal dense systems in the quasistatic limit, shear takes place through a series of rapid rearrangements of particles, avalanches. When the system is trapped in a local minimum of potential energy, the applied strain leads to the increase of energy and shear stress until the minimum becomes unstable. Then, rapid avalanches of rearrangements results in a sharp drop in the energy and shear stress and the system falls in another local minimum. In the over-damped regime, the statistics of such avalanches obey a power-law scaling described with same exponents in $2 \mathrm{D}$ and $3 \mathrm{D}$ systems. When damping is reduced, inertia might carry the system over subsequent energy barriers, reaching a lower minimum in the energy landscape. A different universality class describes the statistics of avalanches in the under-damp regime, where inertia is a key parameter [Salerno and Robbins, 2013; Salerno, Maloney, and Robbins, 2012].

Inertia also affects the flow behavior of systems under steady shear. For instance, the over-damped rheology of dense disordered solids is expressed by the competition between elastic and dissipative forces whose ratio is given by the Weissenberg number. On the other hand, decreasing the damping is found to change the rheology significantly. Nicolas, Barrat, and Rottler, 2016, reported non-monotonic flow curves in the under-damped regime of dense flow. In Chapter 4 we study effects of different dissipation models and inerta in details.

\subsubsection{Friction}

In the presence of friction, particles not only transmit normal forces but also tangential forces, in the case of collision or contact formation. Therefore, it is not a surprise to find friction, changing the physical properties of the system dramatically. For instance, while the yield stress of friction-less systems changes continuously over the jamming transition, flow curves of frictional systems display a discontinuous jump over that transition [Grob, Heussinger, and Zippelius, 2014; Grob, Zippelius, and Heussinger, 2016; Otsuki and Hayakawa, 2011].

In addition, Otsuki and Hayakawa, 2011, showed that in frictional flows, there are three characteristic volume fractions for the jamming transition, with the vanishing distance in the limit of zero friction coefficient. In contrast to friction-less systems, connectivity does not vanish for all $\phi<\phi_{\mathrm{J}}$, force chains exist below the jamming but above 
another critical density $\left(\phi_{c}\right)$. For a given volume fraction between $\phi_{c}$ and $\phi_{\mathrm{J}}$, both inertial and plastic flow can be observed, as well as jammed states. Thus, a more complicated phase diagram is necessary to describe the rheology of frictional systems close to jamming. Figure 1.10 demonstrates such a phase diagram, adapted from [Grob et al., 2014].

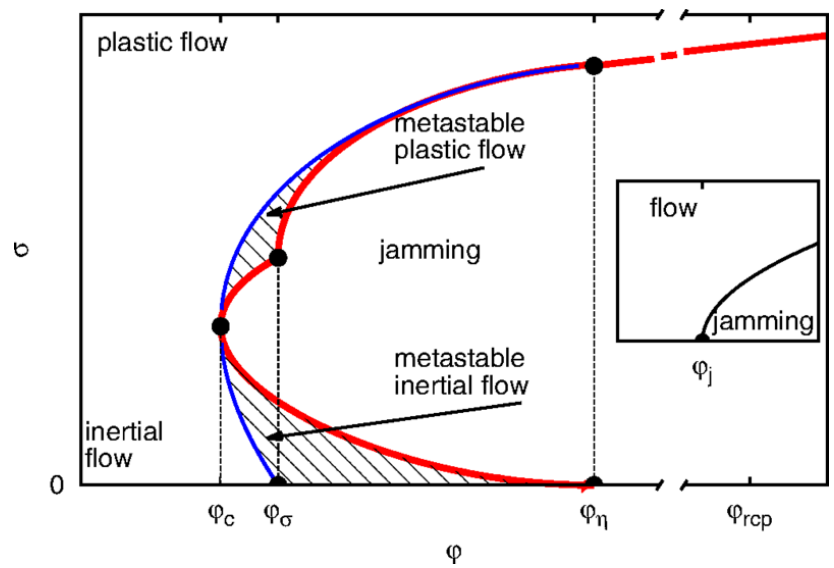

Figure 1.10: Phase diagram for frictional systems. Reentrant flows exist at small and large stress. The figure is adapted from [Grob, Heussinger, and Zippelius, 2014], with the permission.

It is also known that in addition to hydrodynamic interactions, the shear-thickening transition can also be induced by the presence of frictional forces. Such thickening can occur at strain rates which are associated with shear-thinning in friction-less systems [Brown and Jaeger, 2012; Heussinger, 2013].

\subsubsection{Attractive Interactions}

Including attraction in particles interactions might also alter the physical properties of the material significantly. Thermal systems with attractive interactions are found to form repulsive glasses as well as gels and attractive glasses [Eckert and Bartsch, 2002; Pham et al., 2002]. It is a daily observation in beaches that in contrast to dry sand grains, wet sands can be used to build complicated structures as sandcastles or sculptures. Attractive systems below the jamming point are found to exhibit finite yield stress, which is absent in the case of repulsive interactions [Coussot, 2007; Moller et al., 2009; Rahbari et al., 2010]. Lois, Blawzdziewicz, and O'Hern, 2008, reported that there are three regimes of mechanical responses for athermal attractive systems, characterized by two critical transitions, connectivity and rigidity percolation. The latter coincides on the jamming point. Furthermore, the phenomenon of spatially inhomogeneous flows, which is called shear banding, has often been attributed to attractive interactions [Bécu, Manneville, and Colin, 2006; Moller et al., 2009]. Under- 
standing such important and complicated variation in flow behavior, as a consequence of attractive interactions, is the main motivation of this work.

Different circumstances give rise to attractive interactions among particles. Adding some liquid to granular medium might lead to the formation of capillary bridges among grains, stabilizing sandcastles made of wet sand [Fraysse, Thomé, and Petit, 1999; Herminghaus, 2005; Hornbaker D. J. et al., 1997; Mitarai and Nori, 2006]. In fine powders, van der Waals forces induce attraction [Castellanos, 2005]. Adding nonadsorbing polymers to colloidial suspensions can also result in attractive interactions [Ilett et al., 1995; Lekkerkerker et al., 1992].

In Chapter 3, we introduce our simple framework to study weak and short-range attractive interactions. Such interactions can be considered to model cohesive grains or attractive emulsions [Chaudhuri, Berthier, and Bocquet, 2012; Lois et al., 2008].

\subsection{FLOW HETEROGENITIES}

Many soft materials exhibit a phenomenon known as shear banding, in which the material flows in bands with different strain rates, even when the stress field is homogeneous through the whole system [Divoux et al., 2016; Ovarlez et al., 2009; Schall and Hecke, 2010]. In other words, shear-banding refers to the coexistence of high-sheared and low-sheared bands in a flow, where originally one general strain rate is expected. Such flow patterns appear since the material is unable to steadily flow at an applied strain rate.
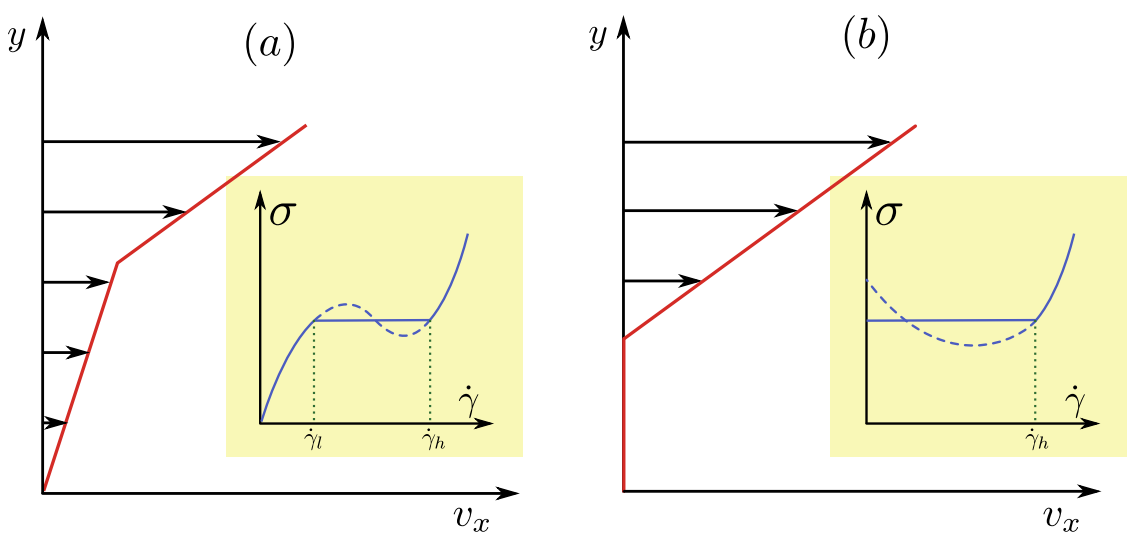

Figure 1.11: Velocitiy fields of a shear banded flow in planer shear geometry and corresponding flow curves. (a) Banded flow with finite values of $\dot{\gamma}_{h}$ and $\dot{\gamma}_{l}$ and (b) a yield stress fluid in a shear-banded state. For yield stress fluids, $\dot{\gamma}_{l}=0$ and the system is shear banded for all $\dot{\gamma}<\dot{\gamma}_{h}$. 
If a fluid exhibits a non-monotonic flow curve (shear stress as a function of strain rate), then the flow is mechanically unstable along the decreasing part of the flow curve [Yerushalmi, Katz, and Shinnar, 1970]. Figure 1.11 displays two possible shear-banded states of a flow, with corresponding non-monotonic flow curves. In analogy with the pressure-volume relation in the case of van der Waals gas, the unstable part needs to be replaced by a stress plateau, and the flow splits into bands bearing high and low strain rates, denoted by $\dot{\gamma}_{h}$ and $\dot{\gamma}_{l}$. These strain rates are connected to bounds of the stress plateau in the flow curve. Different local strain rates in bands, result in different local viscosities in the flow. Thus, the local structure of the flow is also different in bands with different strain rates [Dhont, 1999; Picard et al., 2002].

Coussot and Ovarlez, 2010, proposed a minimal model which attributes shear-banding in jammed systems to situations when the characteristic relaxation time of the system becomes smaller than its restructuring time. For yield stress material (which flow if the applied stress exceeds the yield stress), that condition results in the nonmonotonic flow curve. Interplay between shear-induced destruction of the local structure and its attraction-induced reconstruction can also exhibit non-monotonic flow curves, attributing shear-banding in different materials to attractive interactions [Bécu et al., 2006; Moller et al., 2009]. In Chapter 3 and 4, we will discuss about shear-banding in our attractive systems, thoroughly. 



\section{SIMULATIONS: TECHNICAL DETAILS}

In order to study the rheology of weakly attractive system, we use molecular dynamics to simulate two-dimensional systems of frictionless, soft particles under steady shear with a finite rate. Below, some definitions and technical details are explained.

\subsection{PLANE SHEAR: LEES-EDWARD BOUNDARY CONDITION}

There are two different methods to implement the shearing in planar geometry. One is assuming the moving boundaries as confining walls, while the driving force is used to work on them. The alternative is eliminating walls and the external driving force and using the Lees-Edwards boundary conditions. In the latter case, the periodic images of the system beyond the moving boundaries are also moving, imposing the shear without external force [Lees and Edwards, 1972].

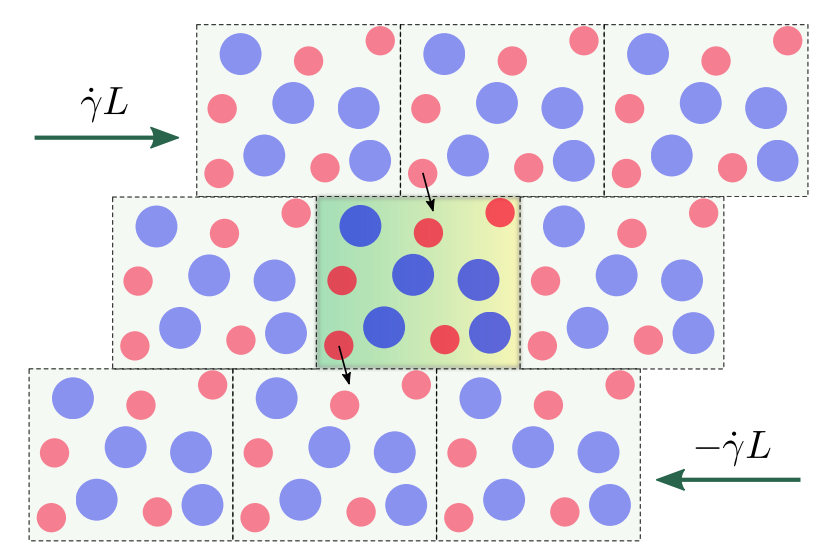

Figure 2.1: Lees-Edwards boundary condition for a system in simple shear flow. The system, represented as a green cell in middle, has normal periodic boundaries is the direction of flow. However, upper and lower periodic images of the system are moving in opposite directions, with constant speeds $\pm \dot{\gamma} \mathrm{L}$, with $\dot{\gamma}$ denoting the applied strain rate and L, being the length of the system in flow gradient direction.

As It is depicted in Figure 2.I, whenever a particle crosses upper or lower boundaries of the simulation box, its position is shifted in the flow direction as

$$
r_{x}^{\text {new }}=r_{x}^{\text {old }} \pm \dot{\gamma} L t
$$


where $\dot{\gamma}$ is strain rate, $\mathrm{L}$ indicates the size of the system in flow gradient direction and $t$, is the shearing time. If the particle goes to an upper periodic image, the shift in its position is positive, otherwise it is negative.

\subsubsection{Imposing Large Deformations}

In practice, the relative distance of the simulation box and vertical images is limited to $-\frac{1}{2}<\frac{\Delta x}{L}<\frac{1}{2}$. This constraint is necessary to avoid extremely tilted boxes, that make simulations inefficient. If a larger deformation is desired, whenever the limiting tilt $\frac{1}{2}\left(-\frac{1}{2}\right)$ is reached, vertical images flip to the other limiting position, $-\frac{1}{2}\left(\frac{1}{2}\right)$. Due to the periodic nature of boundary conditions, all configurations with

$$
\frac{\Delta x}{\mathrm{~L}}=-\frac{1}{2}, \frac{1}{2}, 1, \frac{3}{2}, 2, \ldots
$$

are equivalent. Thus, the system can be sheared until the desired strain is obtained.

\subsection{OBSERVABLES}

\subsubsection{Stress Tensor}

If $\mathrm{d}$ and $\mathrm{V}$ represent respectively the dimension and volume of a given system with $N$ particles, the $\alpha \beta$ component of the stress tensor is written as [Irving and Kirkwood, 1950]

$$
\sigma_{\alpha \beta}^{\text {tot }}=\frac{1}{d V} \sum_{i=1}^{N}\left[-m_{i} \nu_{i, \alpha} \nu_{i, \beta}+F_{i, \alpha} r_{i, \beta}\right] .
$$

The first term in equation above, is the kinetic term or the stress contribution from the flow. There, $m_{i}$ is the mass of $i$ th particle, $v_{i, \alpha}$ and $v_{i, \beta}$ indicate velocity components of the ith particles in $\alpha$ and $\beta$ direction. The second term in Equation 2.2 is known as the virial term, the stress contribution from inerparticle forces. There, while the index $i$ indicates the $i$ th particle, $F_{i, \alpha}$ expresses the $\alpha$ component of the net force $F$ and $r_{i, \beta}$ is the $\beta$ component of the positional vector.

By definition, the pressure tensor is the negative of the stress tensor, expressed in Equation 2.2. Thus, the total pressure of the system can be written as

$$
P^{\text {tot }}=-\frac{1}{d} \sum_{\alpha} \sigma_{\alpha \alpha \prime}^{\text {tot }}
$$

where $d$ is the dimension of the system and the sum is over all unit vectors of the coordination system. Similar to the stress, pressure can also be split into kinetic and virial terms. 
In the limit of small strain rates, the kinetic term in Equation 2.2 becomes negligible; the physics is mainly determined by interactions. On the other hand at large strain rates, total stress diverges from virial stress due to the flow enhancement of the kinetic term. For the rest of this text, whenever we talk about the shear stress, pressure or any stress-related quantity, we use only the virial term unless it is mentioned explicitly. The difference becomes important in Chapter 4, when we discuss the stability of the interface in shear-banded flows.

\subsubsection{Potential Energy}

For a system of $\mathrm{N}$ particles, the average potential energy of the system is defined as

$$
E=\frac{1}{2 N} \sum_{i=1}^{N} \sum_{j=1}^{N} u\left(r_{i j}\right),
$$

where $U$ is the potential interaction and $r_{i j}$, denotes the distance between particles $i$ and $j$. In Chapter 3 , we introduce a simple form of $\mathrm{U}\left(\mathrm{r}_{\mathfrak{i j}}\right)$ to model weakly attractive interactions between soft particles (see Equation 3.1).

\subsection{MEASURMENTS}

In order to setup our simulations, we first obtain a random initial configuration of particles at a given volume fraction. Then, a damped dynamic algorithm is used to minimize the potential energy of the system, eliminating overlaps between particles [Bitzek et al., 2006].

Using Lees-Edwards boundary conditions, the system is sheared with a constant strain rate, $\dot{\gamma}$. Therefore, the strain is proportional to time linearly $\gamma=t \dot{\gamma}$. After reaching the stationary state, where macroscopic observables do not change systematically but fluctuate around mean values, we start measuring desired quantities. Measured observables are averaged over long strain windows with a size depending on the observable and system parameters, $6<\Delta \gamma<100$. 

Part II

RESULTS 



\section{RHEOLOGY OF ATHERMAL SYSTEMS WITH WEAKLY ATTRACTIVE INTERACTIONS}

In the introduction section, we presented a brief review on soft material. We discussed the importance of the flow behavior of such materials and how it is effected dramatically, in the presence of attractive interactions. Here we establish a numerical framework to study rheology of such materials.

In this chapter, we focus on athermal systems under shear, corresponding to shearing granular matter. The typical size of a granular grain is larger than $1 \mu \mathrm{m}$. In such length-scales, the potential energy of the particle with mass $m$ raised by its size in the Earth gravity field is $m g d$, is much larger than $K_{B} T$, the thermal energy-scale at room temperature [Jaeger, Nagel, and Behringer, 1996],

$$
\frac{\mathrm{mgd}}{\mathrm{K}_{\mathrm{B}} \mathrm{T}} \gtrsim 10^{12} \text {. }
$$

Therefore, in the context of granular matter, thermal effects are negligible and athermal models are enough to capture the main physics of the system. Hence, the phase diagram of complex fluids presented in Figure 1.8 is reduced to its athermal plane ( $\sigma$ vs. $1 / \rho$ ) to describe the system (see the left panel in Figure 3.2).

\subsection{THE PARTICLE INTERACTIONS}

We start by introducing a minimal model to include weak attractive interactions in our simulations. $\mathrm{N}$ soft disk particles interact via a harmonic repulsive potential when they overlap. In addition, there is a short-range attractive force between the particles in a particular distance. A parameter $u$ is introduced to characterize the range and also the strength of the attractive force. The inter-particle potential is presented in the following form:

$$
u\left(r_{i j}\right)= \begin{cases}\epsilon\left[\left(1-\frac{r_{i j}}{d_{i j}}\right)^{2}-2 u^{2}\right], & \frac{r_{i j}}{d_{i j}}<1+u \\ -\epsilon\left[1+2 u-\frac{r_{i j}}{d_{i j}}\right]^{2}, & 1+u<\frac{r_{i j}}{d_{i j}}<1+2 u \\ 0, & \frac{r_{i j}}{d_{i j}}>1+2 u\end{cases}
$$

where $r_{i j}$ is the distance between the ith and $j$ th particles, and $d_{i j}=\left(d_{i}+d_{j}\right) / 2$ is the summation of their radii. Figure 3.1 displays 
the potential energy and the corresponding conservative force, where the attractive parts are plotted in green. The attractive force has the similar form as ones used in [Chaudhuri et al., 2012; Lois et al., 2008].

A viscous damping force has been used to keep the system athermal. Next chapter is dedicated to study the importance of the choice of damping force. In the current chapter however, we just use a dissipation model called Contact Dissipation in Normal direction or $\mathrm{CD}_{n}$ model that is described as follows. When two particles overlap (being in contact), $r_{i j}<d_{i j}$, they experience a dissipative force proportional to their relative velocity:

$$
\overrightarrow{\mathrm{r}}_{\text {diss. }}=-\mathrm{b}\left[\left(\vec{v}_{i}-\vec{v}_{j}\right) \cdot \hat{r}_{i j}\right] \hat{r}_{i j},
$$

where $b$ is the damping coefficient. This damping force is directed normally to the contact point, that explains the Normal word in the name. $\mathrm{CD}_{\mathfrak{n}}$ model is typically associated to studies of massive dry granular particles [Shäfer, Dippel, and Wolf, 1996]. In the simulations which are discussed in this chapter, it is chosen that $b=2$ which indicates that our system is always in the over-damped regime. In the next chapter under-damped systems and their novel physics are also investigated.

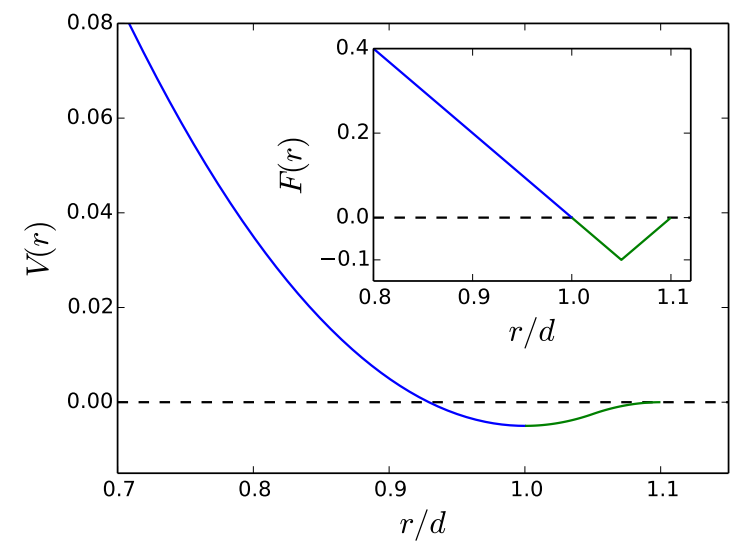

Figure 3.1: Interparticle potential $V(r)$, for $\epsilon=1$ and $u=0.05$. $d$ is the summation of particles radii. The inset shows the corresponding elastic force. The attractive part is shown green.

\subsection{SIMULATION SETUP}

For the simplicity, we just use two-dimensional systems in the simulations which gives us benefits in computational resources. In all simulations, a 50:50 binary mixture of two particles with a relative radii of 1.4 is chosen to avoid crystallization.

The volume fraction is $\phi=\sum_{i=1}^{N} \pi R_{i}^{2} / L^{2}$ where $R_{i}$ is the radius of particle $i$ and $L$ is the system length. A wide range of volume fractions, from $\phi<0.50$ to $\phi=1.0$ has been investigated when jamming 
occurs at $\phi_{\mathrm{J}} \sim 0.8430$. In order to understand the effect of system size, different systems sizes have been studied, $\mathrm{N}=1000,10000$ and 20000.

The unit of energy is determined by $\epsilon$. Thus, the attraction strength is given by $\epsilon u$. The unit of length is the diameter of the smaller particle type, $d=1.0$. The unit of time is hence $d / \sqrt{\epsilon / m}$, where $m=1.0$ is the mass of particles. The velocity-Verlet algorithm is used to integrate the particles' equations of motion. Lees-Edward boundary conditions are used to impose shear with a certain rate, $\dot{\gamma}$.

\subsection{FLOW CURVES}

The reduced jamming phase diagram for athermal repulsive systems is depicted in the left panel of Figure 3.2, while the typical flow curves are presented in the right panel. Above the jamming point, as discussed earlier, the system responses as a disordered solid and in order to make the system flow, a stress larger than a certain threshold, the yield stress, needs to be exerted on the system. The yield stress flows are characterized by a finite stress at the limit of zero strain rate. On the other hand, below the jamming point, imposing any arbitrary stress leads to a finite flow rate.
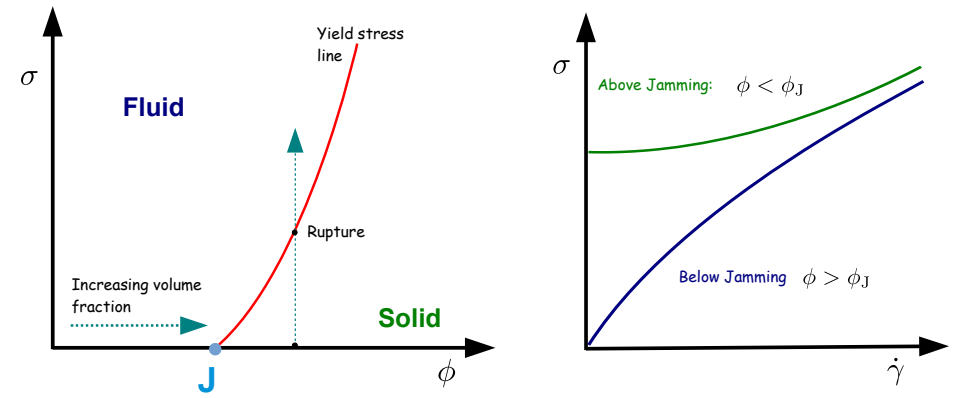

Figure 3.2: (Left), The jamming phase diagram for repulsive systems in the zero temperature plane. (Right), Typical flow curves for a repulsive system in both sides of the jamming point.

By definition, the shear stress has the dimension of $[\mathrm{M}][\mathrm{L}]^{2-\mathrm{D}}[\mathrm{T}]^{-2}$, where $[M],[L]$ and $[T]$ respectively represent the mass, length and time dimensions and $d_{s}$ is the spatial dimension of the system. In the model we proposed for athermal repulsive system subject to the shear with a fixed rate, two time-scales are relevant. One is the shearing time-scale, $\tau_{\dot{\gamma}}=1 / \dot{\gamma}$, which is corresponded to the flow of particles. The other time-scale is the damping time, $\tau_{d}=m / b$, which measures the energy dissipation time-scale during the time particles endure contacts.

The ratio of those time-scales determines the flow regime of particles. If the damping time is very short compared to the shearing time, 
$\tau_{\mathrm{d}} / \tau_{\dot{\gamma}} \ll 1$, the flow is in the inertial regime. Therefore, unless the system is highly dense, the shear stress $\sigma_{x y}$ behaves as

$$
\sigma_{\mathrm{xy}} \sim \mathrm{md}^{2-\mathrm{d}_{\mathrm{s}}} \dot{\gamma}^{2}
$$

which is known as Bagnold scaling [Bagnold, 1954]. On the other hand, if $\tau_{d} / \tau_{\dot{\gamma}} \gg 1$, the flow behavior deviates from Baglond scaling. This regime is called the quasistatic regime, where the stress does not depends on flow-rate related quantities [Campbell, 2002].

However, the above argument is not necessarily valid in attractive systems, since the attraction introduces new energy, time and length scales. Some experiments and also numerical studies show that in attractive systems, a finite yield stress develops below the jamming transition [Coussot, 2007; Lois et al., 2008; Moller et al., 2009; Rahbari et al., 2010]. The effect of attraction on the yield stress for highly dense systems is also studied in [Chaudhuri et al., 2012]. While the yield behavior is expected since the system is highly packed $(\phi=1.0)$, different attraction strength changes the level of stress. We raise the question how the flow curve, $\sigma=\sigma(\dot{\gamma})$, is affected by introducing the attraction both below and above the Jamming transitions.

In Figure 3.3, the left panel presents flow curves obtained by shearing a system with $N=1000$ at $\phi=0.75$ and with different attraction ranges, $u$. The dashed line shows the corresponding flow curve for a repulsive system, $u=0.0$. In such a system the Bagnold scaling determines that shear stress decreases as strain rate decreases. On the other hand, if we define the yield stress as

$$
\sigma_{\mathrm{y}}=\sigma(\dot{\gamma}) \quad \dot{\gamma} \rightarrow 0,
$$

one can see as soon as a finite value sets to the attraction range, a small yield stress appears in the limit of small strain rates. However, in the limit of large strain rates, flow curves reproduce the result for repulsive systems. Thus, attraction affects the system only at small strain rates. The strain rate window in which this change occurs is broadened with increasing the attraction range. We refer to this regime as "attraction-dominated" regime. The rest is called "repulsiondominated" regime.

In the right panel of Figure 3.3, attractive flow curves for different volume fractions $\phi$ and a fixed $u=2 \times 10^{-4}$ have been demonstrated. The finite value of $\sigma_{\mathrm{y}}$ is observed to appear at volume fractions much below the jamming point $\phi_{\mathrm{J}}=0.8430$. Above the jamming point, flow curves follow a standard Herschel-Bulkley form which is consistent with the results presented in [].

Flow curves presented in Figure 3.3 display two interesting features: First, as mentioned before, is the appearance of finite yield stress below the jamming point where the fluid-like behavior is expected for repulsive systems. The second feature is the non-monotonic form of $\sigma=\sigma(\dot{\gamma})$, signaling the possibility of shear-banding which is discussed in details later. 

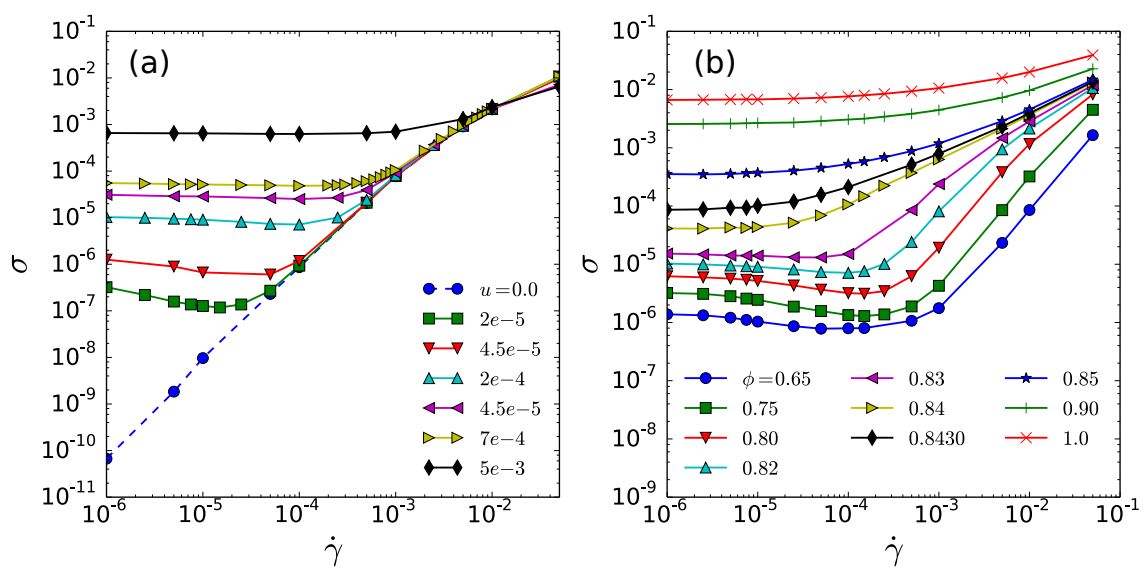

Figure 3.3: Flow Curves, $\sigma=\sigma(\dot{\gamma})$ for (a) different attraction range, $u$, at a fixed volume fraction $\phi=0.82$ and (b) for different volume fractions, $\phi$, at a fixed attraction range, $u=2 \times 10^{-4}$. The dashed line corresponds to the repulsive system, $u=0.0$. For such systems, the jamming transition occurs around $\phi_{\mathrm{J}} \approx 0.8430$.

\subsubsection{Finite Yield Stress}

As it is depicted in Figure 3.3 (right), finite yield stress exists even far below the jamming point, where zero yield stress is expected for repulsive systems. A valid question is that at what volume fraction the rigidity transition occurs in such systems? Is there a threshold in volume fraction above which the shear rigidity sets in for weakly attractive systems? To answer this question one needs to go to smaller $\phi$ than what we show in Figure 3.3. For $\phi<0.50$ the system shows strong sensitivity to the initial configuration and defining a steady state to measure the yield stress is difficult. In such systems, particles frequently form a large cluster and flow for a while, or form two or more smaller clusters which do not interact most of the time, due to the large void spaces between them(see Figure 3.4). These two possibilities can be converted to each-other during a simulation time. Therefore the stress measurement would be highly sensitive to the strain window at which the measurement is performed. To avoid such technical problems, we focus on denser systems $(\phi \geqslant 0.50)$.

\subsubsection{Non-monotonic behavior}

Looking at attraction-dominated regime, one can see in Figure 3.3 a non-monotonic behavior in flow curves below the jamming point. In the range of attraction strength that we investigated and for $\phi<\phi_{\mathrm{J}}$, there exists a strain rate window where stress decreases with strain rate. This is similar to the non-monotonic dependence of pressure $p$ to volume $v$ in the Van der Waals equation for gases. Figure 3.5 displays a typical $p(v)$ curve for an isotherm van der Waals gas. The 

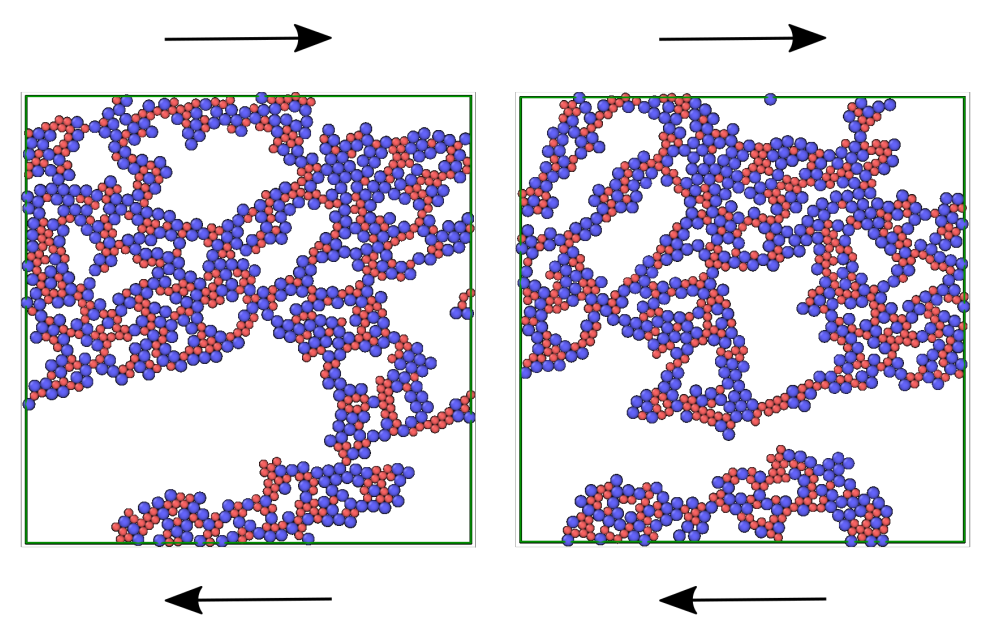

Figure 3.4: Two snapshots of a dilute system with $N=1000, u=2 \times 10^{-4}$ and $\phi=0.40$. (Left), All particles form a large cluster and flow due to shearing. (Right), Later in the same system, particles form two isolated clusters which do not interact for a long time. Arrows indicate the shearing direction.

non-monotonic part which is shown in red color, is mechanically unstable and needs to be replaced by an iso-pressure line obtaining from the Maxwell equal area construction. Along that line, phase-coexistence occurs.

In the shearing systems we study, the same situation happens. The part in flow curves with $d \sigma / d \dot{\gamma}<0$ is not mechanically stable. Thus at least from theoretical point of view, non-monotonic flow curves lead to mechanical instability and as a phase coexistence, localized shear bands form in the corresponding systems (bands with different $\dot{\gamma}$ but similar $\sigma$ ) [Dhont, 1999; Picard et al., 2002]. However, no shear bands is observed in our systems with $N=1000$, and the flow stays stable because of the small system size [Dhont, 1999]. In Section 3.11, using linear stability analysis, we will discuss how small system sizes prevent shear-banding in system with non-monotonic flow curves. There, we will also study shear localization in larger systems, and properties of shear bands, in details.

\subsection{PHASE DIAGRAM}

Regarding the yield stress emergence and the non-monotonic behavior of the flow curve below the Jamming point, the jamming phase diagram needs to be modified for attractive systems. The left panel in Figure 3.6 shows such a modified phase diagram in the $(\sigma, \phi)$ plane. Above the jamming point, $\phi>\phi_{\mathrm{J}}$, flow curves are monotonic, the yield stress is independent of $u$ and scales as $\sigma_{y}^{(\text {rep })} \propto\left(\phi-\phi_{\mathrm{J}}\right)^{\alpha}$. We found $\alpha \approx 1.04$ which is consistent with the reported value for the 


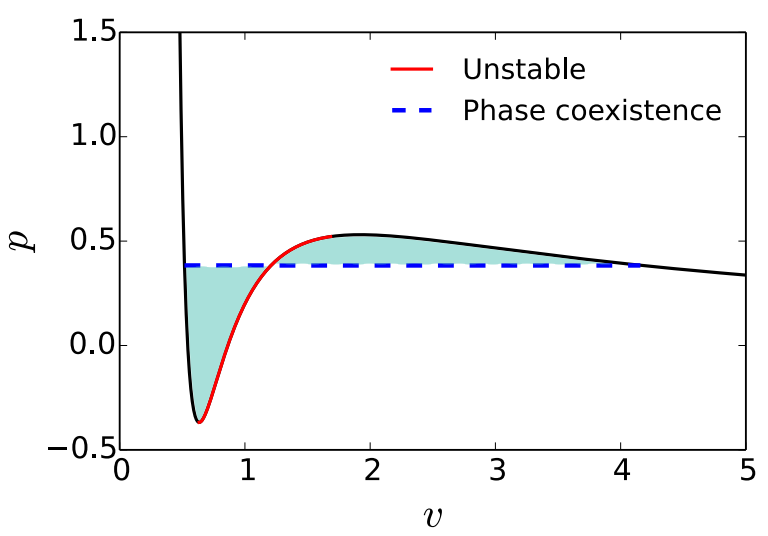

Figure 3.5: A typical $(p, v)$ diagram of the van der Waals gas with the reduced equation of state as $\left(p+\frac{3}{v^{2}}\right)(3 v-1)=8 t$, at the constant temperature $t=0.8$. The red line indicates the non-monotonic part, where $\partial \mathrm{p} / \partial v>0$ and the system is not stable. The dashed line is the phase coexistence line, obtained from the Maxwell equal area construction, replacing the two shaded regions with equal area. Along that line, two phases of the system, gas and liquid, coexists with the same pressure.

repulsive particles [Heussinger, Chaudhuri, and Barrat, 2010]. This value for $\alpha$ might be affected by finite size effects [Olsson and Teitel, 2011].

In contrast to repulsive systems and as a consequence of finite attraction, the system exhibits solid-like behavior (finite $\sigma_{y}$ ) below the jamming point even at the smallest $\phi$ that we have explored, $\phi=0.50$. The corresponding solid region in the phase diagram is separated from the flow region by the yield stress line which does not exist for $\phi<\phi_{\mathrm{J}}$ in repulsive systems, where the shear rigidity sets in at $\phi_{\mathrm{J}}$. In Figure 3.6 (left) the yield stress line for $u=2 \times 10^{-4}$ is plotted. In addition, a dashed line determining the regime where the system flows corresponding to the non-monotonic part of the flow curve is illustrated. For each $\phi$, this line marks the location of the minimum in the non-monotonic flow curve. Thus, in this regime, steady state shear banding is expected.

The left panel of Figure 3.3 shows that the non-monotonicity in flow curves disappears with increasing attraction strength. So, a valid question rises that how the threshold of attraction, at which such flow instabilities do not appear, changes as a function of $\phi$. In order to answer this question we obtained the corresponding phase diagram of the system in $(u, \phi)$ plane, presented in the right panel of Figure 3.6. This threshold decreases with decreasing $\phi$. For a system at $\phi=0.70$ we observed such non-monotonic behavior for all the attraction strengths that we explored $\left(2 \times 10^{-5} \leqslant u \leqslant 5 \times 10^{-2}\right)$. 

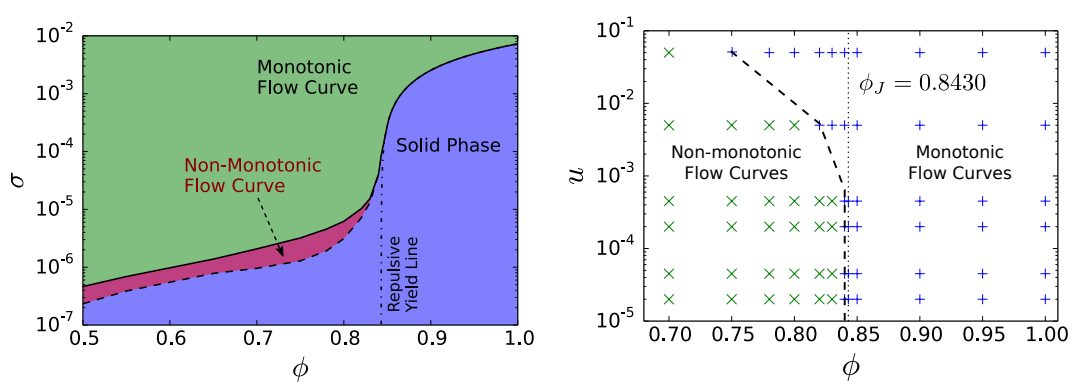

Figure 3.6: (Left), The jamming phase diagram for weakly attractive systems: The black line indicates $\sigma_{y}(\phi)$ for $u=2 \times 10^{-4}$. Above the Jamming point, yield stress behaves as $\sigma_{y} \propto \delta \phi^{\alpha}$ where we found $\alpha=1.04$. Below the jamming point $\phi_{\mathrm{J}} \approx 0.8430$, finite $\sigma_{y}$ and also non-monotonic flow curves are observed. (Right), The phase space of $u$ and $\phi$, where points to observe the nonmonotonic (cross symbols) or monotonic (plus symbols) flow curves are determined. The threshold $\phi$ of the transition between non-monotonic and monotonic flow curve decreases with the attraction strength.

\section{$3 \cdot 5$ CONNECTIVITY}

A pair of particles are in contact if their distance is in the range of the pair potential. If $z_{i}$ indicates the number of contacts for ith particle in the system, we can define the average coordination number or connectivity of the system, $z$, as

$$
z=\frac{1}{\mathrm{~N}} \sum_{\mathrm{i}=1}^{\mathrm{N}} z_{\mathrm{i}}
$$

Looking at connectivity $z$, provides more information about the micro-structure of the system.

\subsubsection{Isostatic point}

The isostatic point is the point at which particles in the system has the minimum number of contacts to form a system spanning structure which is mechanically stable. The connectivity of the system at the isostatic point is shown by $z_{\text {iso }}$. For the systems showing rigidity, like a system above the jamming point, it is necessary to have

$$
z \geqslant z_{\text {iso }}
$$

while in the context of jamming transition, $z=z_{\text {iso }}$ happens exactly at $\phi=\phi_{\mathrm{J}}$.

The fact that at the isostatic point, all the forces are balanced due to the contacts, helps us to calculate $z_{\text {iso. }}$. If the forces are not balance, there would be some movement like rearrangements to reach the equilibrium. For a system with the average connectivity $z$, there 
are $\mathrm{Nz} / 2$ constraints coming from contact forces (each contact involves two particles). On the other hand, there are $\mathrm{N}$ particles in a d-dimensional system with $\mathrm{Nd}$ force equations. At the isostatic point, the number of equations and constraints should be the same:

$$
\frac{\mathrm{N} z}{2}=\mathrm{Nd}
$$

which gives the $z_{\text {iso }}$ as

$$
z_{\text {iso }}=2 \mathrm{~d} \text {. }
$$

This is known as Maxwell criterion for mechanical stability, established by J. C. Maxwell in 1864[Maxwell, 1864].

For repulsive systems, $z$ shows a discontinue transition at the jamming transition. For static systems with repulsive interactions, $z=0$ at $\phi<\phi_{\mathrm{J}}$, and it scales as

$$
z-z_{\mathrm{iso}} \sim\left(\phi-\phi_{\mathrm{J}}\right)^{1 / 2}
$$

above the jamming point [O'Hern et al., 2003].

\subsubsection{New Type of Connectivity}

In our model, with the finite range of attraction $u$, it is reasonable to define a new type of contact. The left panel of Figure 3.7 visualizes the idea of new type of contacts on the force diagram. Whenever particles interact via the repulsive term of equation 3.1, which corresponds to

$$
r_{i j}<d_{i j}
$$

the contact is repulsive. Following the same argument, when

$$
1<r_{i j} / d_{i j}<1+2 u
$$

which corresponds to particles interacting via an attractive force, the contact is also attractive. Average repulsive and attractive connectivity can then be defined as the average number of repulsive and attractive contacts, indicated by $z_{\text {rep }}$ and $z_{\mathrm{att}}$. The total connectivity of the system is now defined as

$$
z=z_{\text {rep }}+z_{\text {att }}
$$

Figure 3.7 shows how the repulsive and attractive coordination numbers vary with strain rate as well as the total coordination number for a system with $u=2 \times 10^{-4}$ and $\phi=0.75$. At small strain rates, both repulsive and attractive connectivity are constant with larger number of particle pairs with $r_{i j} \leqslant d_{i j}$. Increasing the strain rate, $z_{\text {att }}$ rapidly decays as attractive contacts break apart with increasing $\dot{\gamma}$ and become negligible in the repulsion-dominated regime. On the 

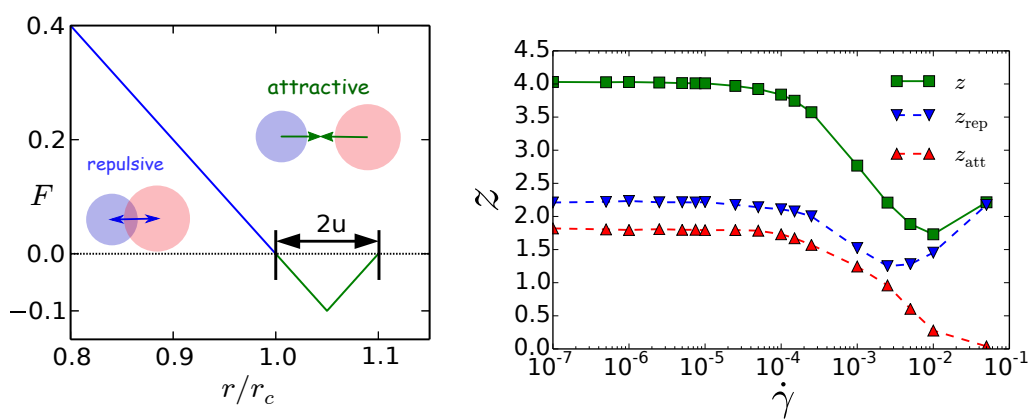

Figure 3.7: Different kind of connectivity as a function of strain rate for a system at $\phi=0.75$ and $u=2 \times 10^{-4}$. At small strain rates, attractive contacts play important role and keep the total connectivity larger than the isostatic value. at large strain rates, repulsive contacts are dominant, determining the total connectivity behavior. The drop in $z(\dot{\gamma})$ from isostatic point to the value much smaller, indicates the attractive timescale $\tau_{a}$.

other hand, $z_{\text {rep }}$ exhibits a non-monotonic behavior. While contacts get initially disrupted and thereby decrease by increasing the strain rate, particles once again get pushed together when the repulsiondominated regime kicks in. Thus, at large strain rates, $z$ is mainly indicated by $z_{\text {rep. }}$.

\subsubsection{Rigidity Below the Jamming Point}

It is already known that for repulsive systems below the jamming point $\phi<\phi_{\mathrm{J}}$, where no rigidity develops, the connectivity is significantly smaller than $z_{\text {iso }}$. How does the presence of attraction affect the formation of contacts and value of $z$ ? Figure 3.7 shows such a situation, where $z(\dot{\gamma})$ is plotted at $\phi=0.75$ : despites the fact that the system is below the jamming $\operatorname{point}\left(\phi_{\mathrm{J}}=0.8430\right)$, the total connectivity in the limit of small $\dot{\gamma}$ is almost the same as an isostatic system, $z \approx z_{\text {iso. }}$.

This behavior is more apparent in Figure 3.8, which exhibits $z(\dot{\gamma})$ for different $\phi$ from far below the jamming point $(\phi=0.65)$ to highly dense $\operatorname{system}(\phi=1.0)$. The dashed line represents the connectivity in a repulsive system at $\phi=0.65$ and solid lines correspond to attractive systems with $u=2 \times 10^{-4}$. In the limit of small strain rates, it is observed that $z(\dot{\gamma}) \rightarrow 0$ at $\dot{\gamma} \rightarrow 0$ for repulsive particles below the jamming, which is expected for our model of particles dynamics [Vågberg, Olsson, and Teitel, 2014]. But in attractive systems and below the jamming, as soon as the attraction is introduced, $z_{y} \equiv z(\dot{\gamma} \rightarrow 0)$ jumps to a value slightly larger than the isostatic connectivity $z_{\text {iso }}=4$. Figure 3.8 reveals that such a behavior holds for $\phi$ even far below $\phi_{\mathrm{J}}$. Therefore, finite but small attraction for systems at $\phi \ll \phi_{\text {J }}$ results in similar isostatic structures as the structure for $\phi=\phi_{\mathrm{J}}$ in repul- 


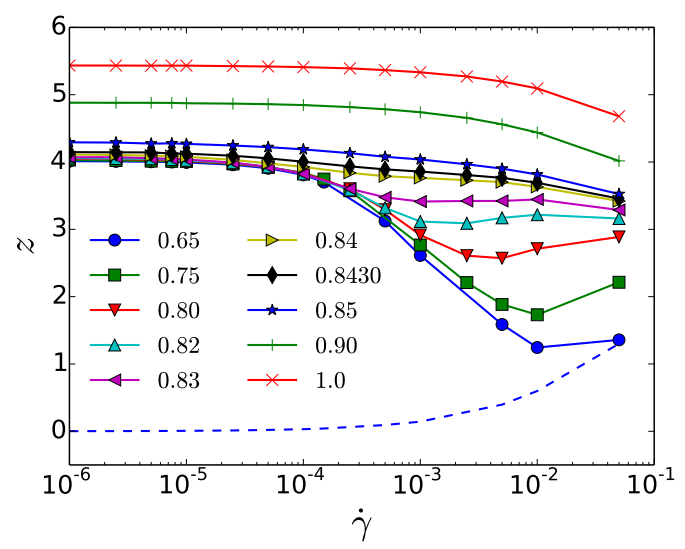

Figure 3.8: $z(\dot{\gamma})$ for different volume fractions. The dashed line corresponds to the repulsive system $u=0.0$, solid lines represent attractive system with $u=2 \times 10^{-4}$.

sive systems. This structure is responsible for the appearance of finite yield stress in attractive systems below the jamming point, presented in Figures 3.6 and 3.3.

As one can see in Figures 3.8 and 3.7, at the limit of small strain rates, $z(\dot{\gamma})>z_{\text {iso. }} z(\dot{\gamma})$ remains almost constant with $\dot{\gamma}$ until at a special strain rate, it drops to a value far below $z_{\text {iso }}$. This behavior of connectivity indicates that at small strain rates, attractive forces keep the nearly-isostatic structure of the system stable until the point where the strain rate is fast enough to destroy the structure. This strain rate gives us an attractive time-scale $\tau_{a}$ which is found to scale with the attraction range as:

$$
\tau_{\mathrm{a}} \sim 1 / \mathrm{u}
$$

Figure 3.9 displays $\delta z=z_{y}-z$ as a function of rescaled strain rate $\dot{\gamma} \tau_{\mathrm{a}}$, for different values of $u$ and $\phi<\phi_{\mathrm{J}}$. Scaling the strain rate by $\tau_{a}$, collapses all $\delta z$ obtained from the attraction-dominated regime on a single curve.

For $\phi>\phi_{\mathrm{J}}$ we find $z_{\mathrm{y}}-z_{\text {iso }}=\zeta_{\mathrm{o}}\left(\phi-\phi_{\mathrm{J}}\right)^{1 / 2}$ with $\zeta_{0} \approx 3.78$, consistent with Equation 3.9. In the limit of large strain rates, $z(\dot{\gamma})$ reproduces the repulsive results as it is shown in Figure 3.8 for a system at $\phi=0.65$.

\subsubsection{Fragile Solid: Yield Stress and Connectivity}

Now we explain the connection between yield stress and connectivity of the system below the jamming point. Lets assume $\delta$ represents the relative displacement of neighboring particles. It can be decomposed into components which are perpendicular $\left(\delta_{\perp}\right)$ and parallel $\left(\delta_{\|}\right)$to the contact line. It is known [Hecke, 2010] in elastic spring networks and systems of soft repulsive particles, that the linear response close 

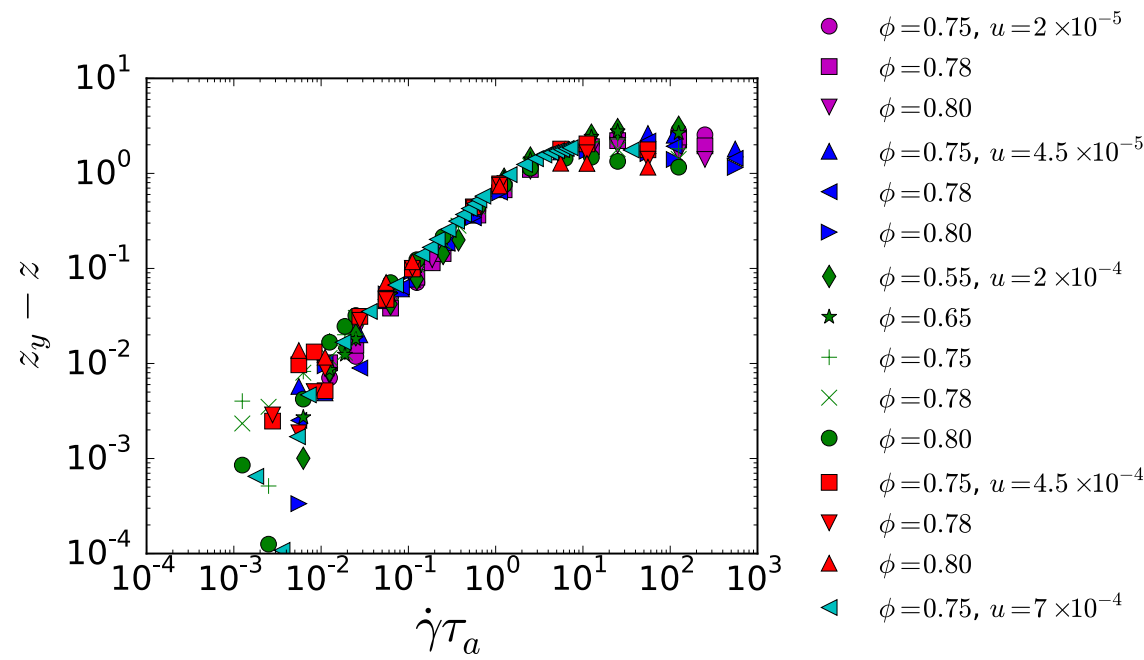

Figure 3.9: The distance to the yield connectivity $\delta z=z_{y}-z$, versus rescaled strain rate $\dot{\gamma} \tau_{\mathrm{a}}$. Rescaling the strain rate with attractive timescale, collapse all $\delta z$ obtained from different values of $u$ and $\phi<\phi_{\mathrm{J}}$ on a single curve. While different colors indicated different $u$, symbols represents different $\phi$.

to the isostatic point is characterized by strong non-affine motion, expressed by relative particle displacements,

$$
\delta_{\perp} \sim \frac{\gamma}{\delta z^{1 / 2}}
$$

$\delta z=z-z_{\text {iso }}$ expresses the distance to the isostatic point. Such displacements are directed tangentially to the particle contact. The corresponding shear modulus to such a linear-elastic response reads as

$$
g_{\text {lin }} \sim \delta z
$$

In our system when the contact is not broken, particles see each other through the harmonic force with a range determined by $u$. Thus for motion amplitudes smaller than this range our system can be considered as a network of elastic springs and yielding can be defined as the point where the motion amplitude exceeds the range of attraction and breaks the contact.

To determine the yielding point, we use the non-linear loading conditions where the particle's tangential motion is associated with higher-order longitudinal contribution (Pythagoras) [Lieleg et al., 2007; Wyart et al., 2008]:

$$
\delta_{\|} \sim \frac{\delta_{\perp}^{2}}{R}
$$

In our attractive system, the maximum dilation strain should be smaller than the attraction range, $\delta_{\|}<u$ (Figure 3.10). Thus, putting Equation 3.12 in Equation 3.14 gives the yield strain as

$$
\gamma_{\mathrm{y}} \sim(u \delta z)^{1 / 2} \text {. }
$$




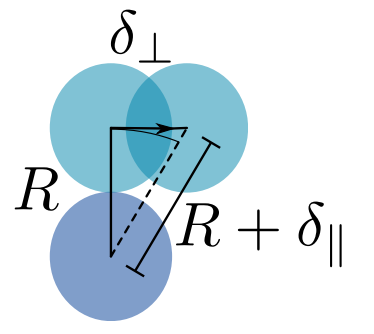

$\delta_{\|} \sim \delta_{\perp}^{2} / R$

Yielding Point:

$$
\delta_{\|}=u
$$

Figure 3.10: Sketch of a pair of particles at the yielding point.

Since we have $\sigma_{\mathrm{y}} \sim \mathrm{g}_{\text {lin }} \gamma_{\mathrm{y}}$, one can write the following scaling relation for the yield stress, attraction range and the distance to the isostatic point:

$$
\sigma_{y} /|\delta \phi|^{\alpha} \propto \begin{cases}u^{1 / 2} \delta z^{3 / 2} /|\delta \phi|^{\alpha}, & \sigma_{y}^{\text {(rep })} \ll \sigma_{y}^{(\text {att })} \\ 1, & \sigma_{y}^{\text {(rep })} \gg \sigma_{y}^{\text {(att })} .\end{cases}
$$

Weak attractive forces as we use in our model result in formation of weak solid (nearly-isostatic network of particles) below the jamming point. The mechanical response of this weak solid is explained by Equation 3.16. Figure 3.11 shows that Equation 3.16 holds nicely for systems with a wide range of volume fractions below the jamming point and different attraction ranges (Dashed line). Above the jamming point, the well-known repulsive behavior $\sigma_{y} \sim|\delta \phi|^{\alpha}$ is observed (Figure 3.11: Dotted line). This can be understood by noting the fact that in highly dense systems, the repulsive term of Equation 3.I is dominated.

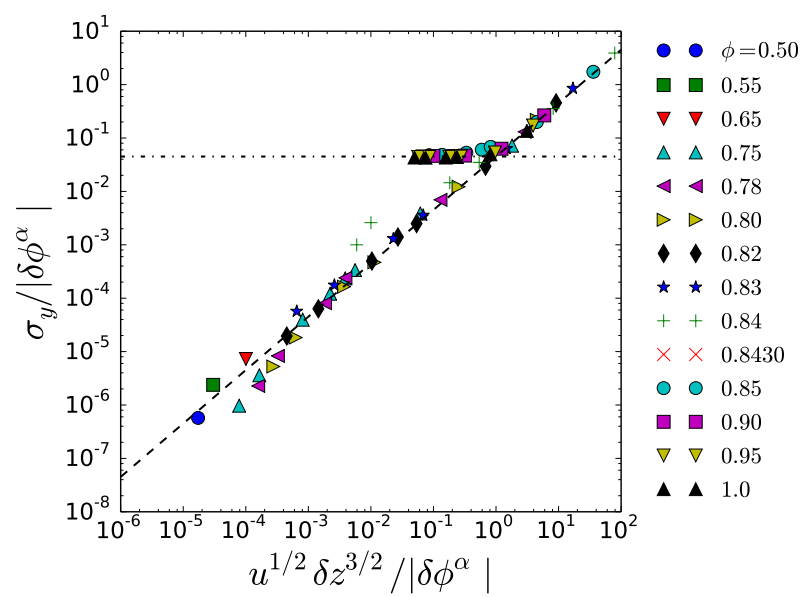

Figure 3.11: Yield stress $\sigma_{y}$ normalized by $|\delta \phi|^{\alpha}$ as a function of the combination $u^{1 / 2} \delta z^{3 / 2}$. For $\phi<\phi_{j}$, Equation 3.16 holds. For $\phi>\phi_{j}$ (Dashed line), yield stress shows the expected repulsive behavior $\sigma_{y} \sim|\delta \phi|^{\alpha}$ (Dotted line). 
At small $\dot{\gamma}$, relaxation time is much smaller than shearing time scale which leads to the continuous reconstruction of the fragile solid. On the other hand at large $\dot{\gamma}$ this local structure breaks down due to fast shearing and relatively large relaxation time. The competition between these two mechanisms, attraction induced aggregation and shear induced rupture of local structure, explains the decrease of stress at the intermediate regime or in other words, the non-monotonic behavior of the flow curve. In the Section 3.11, we will discuss about this behavior and its consequences in more details.

\subsection{POTENTIAL ENERGY}

The attractive force in the model we study, introduces a new energyscale to the system, which is quantified as

$$
\mathrm{E}_{0}^{(\mathrm{att})} \sim \epsilon \mathrm{u}^{2}
$$

We study the average potential energy of the system, E, to understand how this new energy-scale show up during the rheology. The potential energy of the system is defined as

$$
\mathrm{E}=\frac{2}{\mathrm{~N} z} \sum_{i, j} \mathrm{~V}\left(\mathrm{r}_{i j}\right),
$$

where $r_{i j}$ is the distance between particles $i$ and $j, V\left(r_{i j}\right)$ obtained from Equation 3.I, is the corresponding potential energy of the contact between that pair of particles and $\mathrm{Nz} / 2$ indicates the number of contacts in the system.

Figure 3.12 shows $E=E(\dot{\gamma})$ for different $\phi$. The dashed line corresponds to the repulsive system at $\phi=0.65$ and solid lines represent systems with $u=2 \times 10^{-4}$. Above the jamming, $\phi>\phi_{\mathrm{J}}$, the repulsive term from Equation 3.I is dominated thus $E(\dot{\gamma})$ reproduces the repulsive results. On the other hand, in the attraction-dominated regime, most of the particles make contacts influenced by attraction. Hence, the attractive potential energy is written as

$$
\mathrm{E} \sim-\epsilon \mathrm{u}^{2} \mathrm{~N} z(\dot{\gamma}) / 2
$$

The quantity $\mathrm{N} z(\dot{\gamma}) / 2$ gives the number of contacts as a function of the strain rate. Figure 3.7 shows that $z(\dot{\gamma})$ is almost constant in the small $\dot{\gamma}$ regime, below the jamming point. Further, it also does not vary much with $\phi$ (see Figure 3.8). Thus, in this regime, equation 3.19 explains that the potential energy is expected to be constant, which is illustrated in the bottom panel of Figure 3.12.

Similar to defining a stress threshold for yielding to occur, one can define a yield potential energy as

$$
\mathrm{E}_{\mathrm{y}}=\mathrm{E}(\dot{\gamma}) \quad \dot{\gamma} \rightarrow 0 .
$$




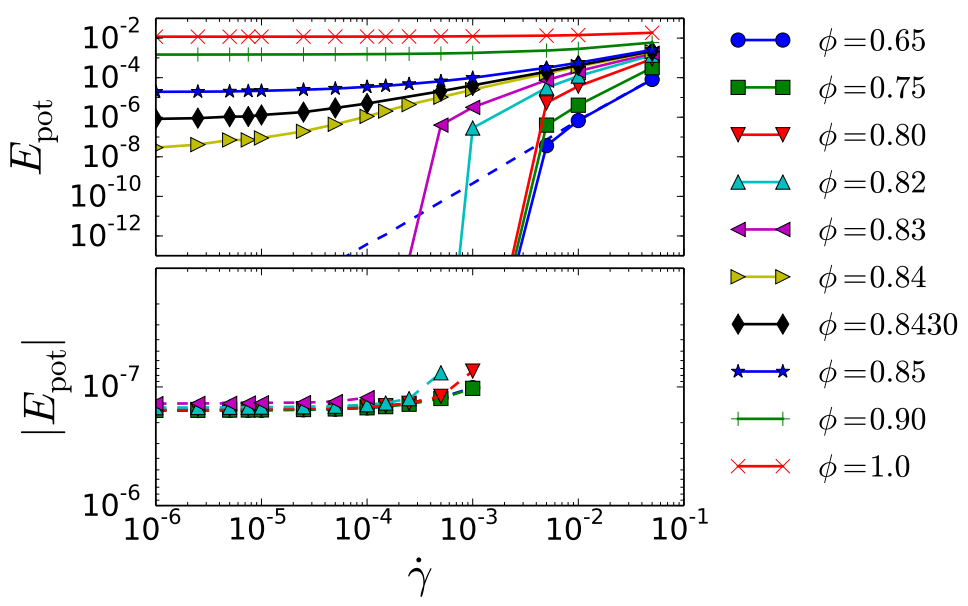

Figure 3.12: Potential Energy $E$ as function of strain rate $\dot{\gamma}$ for different volume fractions. The dashed line represents repulsive system with vanishing energy at the limit of zero strain rate. Solid lines are associated with attractive systems with the attraction range $u=2 \times 10^{-4}$.

The variation of the estimated $E_{y}$ with $\phi$ is shown in Figure 3.13 for different attraction strengths. Above the jamming point, where repulsion dominates, the yield energy grows as $E_{y}^{(\text {rep })} \propto\left(\phi-\phi_{J}\right)^{\beta}$ where we find $\beta \approx 2.1$. It is known for purely repulsive particles that the energy scales with stress as $E \propto \sigma^{2}$. Therefore it is expected to find $\beta=2 \alpha$ which is consistent with our observations.

Below the jamming point, where attraction dominates, it is expected from Equation 3.19 that $\left|E_{y}\right| \sim u^{2}$ (see section (c) in Figure 3.13).
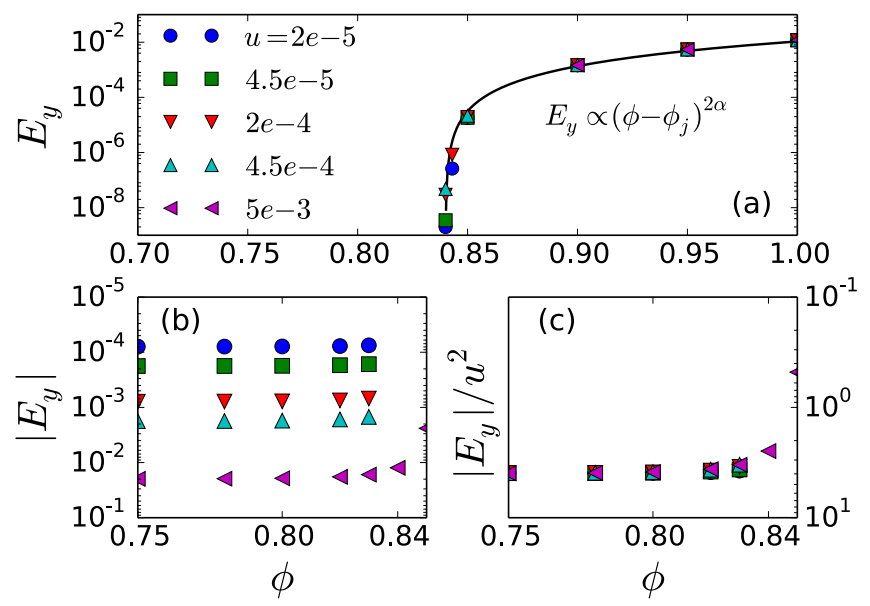

Figure 3.13: Yield potential energy $E_{y}$ as a function of $\phi$ for different attractions $u$ in (a) repulsion-dominated regime, $\phi>\phi_{\mathrm{J}}$ and (b) attraction-dominated regime, $\phi<\phi_{\mathrm{J}}$. (c) Scaled $\mathrm{E}_{\mathrm{y}}(\phi)$ by $\mathrm{u}^{2}$ in the attraction-dominated regime. 


\subsection{SHEAR STRESS RATIO}

The nearly-isostatic solid formed due to attractive interactions at $\phi<$ $\phi_{\mathrm{J}}$, provides resistance to plastic deformations under shear. In the context of shearing granular matter, this macroscopic resistance (or friction) is quantified via the ratio of shear-stress to pressure:

$$
\mu=\frac{\sigma_{x y}}{P} .
$$

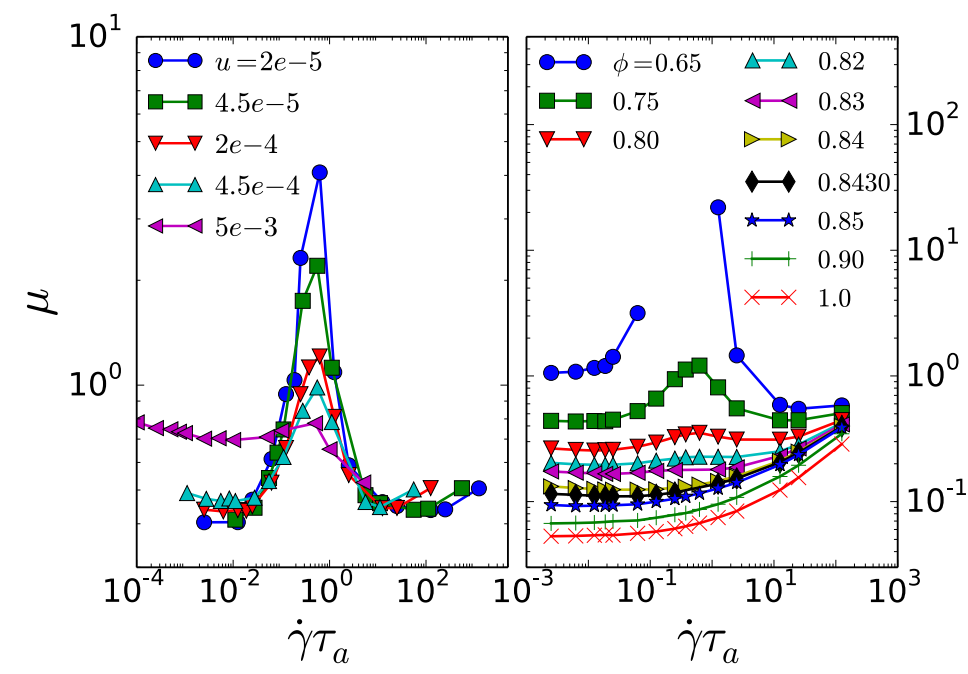

Figure 3.14: Shear-stress ratio as a function of $\dot{\gamma}$ for different attraction ranges at $\phi=0.75$ (left panel) and for different volume fractions at $u=2 \times 10^{-4}$. Note that for $\phi<\phi_{\mathrm{J}}$, the maximum peak appears close to $\dot{\gamma}^{*}$ where $\sigma\left(\dot{\gamma}^{*}\right)$ is minimum.

Left panel in Figure 3.14 shows data for $\mu$ as a function of strain rate, for a system at $\phi=0.75$ and changing attraction strength. Right panel in Figure 3.14 shows $\mu(\dot{\gamma})$ for a fixed attraction $\left(u=2 \times 10^{-4}\right)$ but different volume fractions. In both panels the strain rate is rescaled with the attractive time-scale $\tau_{a}$. The yield stress ratio, or the resistance of the system at the limit of vanishing strain rate, can be also defined as

$$
\mu_{y}=\mu(\dot{\gamma}) \quad \dot{\gamma} \rightarrow 0 .
$$

In the left panel of Figure 3.14, we observe that the yield stress ratio increases with attraction strength. This is expected since attraction results in particle clustering which is not in favor of flowing process, leading to the stronger resistance [Gu, Chialvo, and Sundaresan, 2014; Rognon et al., 2008].

It can also be seen in Figure 3.14 that for $\phi<\phi_{\mathrm{J}}$ and finite $u$, $\mu(\dot{\gamma})$ develops a peak at a strain rate close to $\dot{\gamma}^{*}$, where the minimum occurs in flow curves $\sigma^{\mathrm{min}}=\sigma\left(\dot{\gamma}^{*}\right)$ (see Figure 3.3). In fact, rescaling of $\dot{\gamma}$ by $\tau_{a}$, results in the location of the peak to be at the same $\dot{\gamma}^{*}$ for 
different $u$, with the peak hight decreasing with increasing attraction. Figure 3.15 shows the yield stress ratio and the height of the peak in $\mu(\dot{\gamma})$ as functions of $u$. In the right panel of Figure 3.14, there is a discontinuity in $\mu\left(\dot{\gamma} \tau_{a}\right)$ at $\phi=0.65$ which is explained in below.

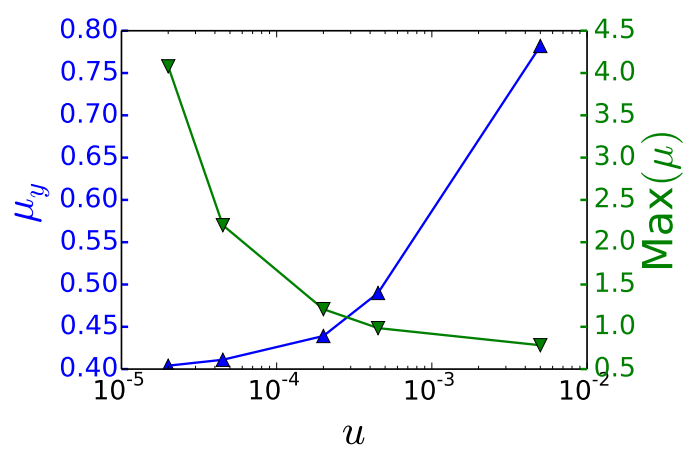

Figure 3.15: For a system at $\phi=0.75, \mu_{y}$ (blue curve) and the height of the maximum peak in $\mu(\dot{\gamma})$ (green curve), are plotted as functions of attraction strength, $u$.

To aid the discussion on macroscopic resistance, we also present the data for pressure in Figure 3.16, for the same set of $\phi$ for which shear stress is shown in Figure 3.3. Pressure displays a similar nonmonotonic behavior as shear stress, the quantitative comparison of which is captured by $\mu$. The striking observation is that at $\phi=0.65$ the pressure curve becomes discontinues on the log-scale, in the regime where it is non-monotonic. In that window, the pressure is actually negative implying the dominance of internal tensile forces within the system.

For $\phi<\phi_{\mathrm{J}}$, the non-monotonic behavior of $\mu(\dot{\gamma})$ is observed and disappears for $\phi>\phi_{\mathrm{J}}$. Recalling the discussion above, the apparent discontinuity of $\mu$ for $\phi=0.65$ is due to the pressure becoming negative. The non-monotonic behavior of $\mu$ is observed since, under shear, the pressure of the particle assembly drops faster than the shear stress. This results in changes in micro-structure of the system, which is discussed in the next section.

\subsection{STRUCTURE FACTOR}

In order to study the local structures of the system in attractiondominated regime, a natural quantity to measure is the structure factor of the system, $S(q)$, which is defined as:

$$
S(q)=N^{-1}\langle\rho(q) \rho(-q)\rangle=\frac{1}{N} \sum_{i, j}^{N} e^{i q\left(r_{i}-r_{j}\right)}
$$

with $N$ as the number of particles and $r_{i}, r_{j}$ being the position of the $i$ th and $j$ th particle respectively. The quantity $\rho(q)=\sum_{i}^{N} e^{i \mathbf{q} \cdot \mathbf{r}_{\mathbf{i}}}$ 


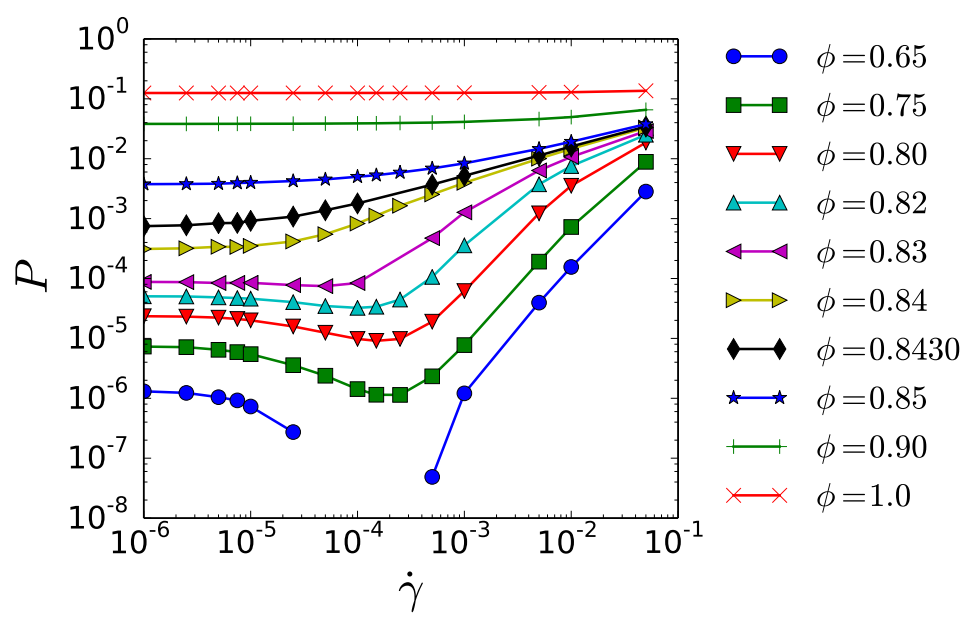

Figure 3.16: Pressure P as a function of strain rate $\dot{\gamma}$ for different volume fractions. The attraction range is $u=2 \times 10^{-4}$. The discontinuity in $\mathrm{P}(\dot{\gamma})$ at $\phi=0.65$ corresponds to negative values of $\mathrm{P}$ (compressive pressure).

is the Fourier transform of the number density. The structure factor basically measures the strength of density fluctuations at a length scale l, where

$$
l=\frac{2 \pi}{|\mathbf{q}|}
$$

Figure 3.17 shows $S(q)$ at $u=2 \times 10^{-4}$ and $\phi=0.75$ for different $\dot{\gamma}$. One can observe a small peak at $\mathrm{q}<2$ in the attraction-dominated regime which is not as enhanced in the repulsion-dominated regime. If the peak appears at $\mathrm{q}^{*}$, we define $\mathrm{S}^{*}=S\left(\mathrm{q}^{*}\right)$. This peak corresponds to the local structure formation in the limit of small but finite strain rates.

Figure 3.18 provides two snapshots of the system from the attractiondominated $(\dot{\gamma}=1 e-6)$ and repulsion-dominated regime $(\dot{\gamma}=1 e-3)$ to compare. In the left panel, formation of a local structure, small clusters and voids, is clear. In the limit of slow shearing, attractive forces help particles to form and maintain these clusters, leading to the formation of an isostatic network of particles that we discussed before. The small peak in the structure factor, $\mathrm{S}^{*}$, rises due to this structure, introducing the new length-scale, $l^{*}$ :

$$
l^{*}=\frac{2 \pi}{q^{*}}
$$

which indicates the cluster size. For the system presented in the Figure 3.18 (a) $\left(\phi=0.75, u=2 \times 10^{-4}\right.$ and $\left.\dot{\gamma}=1 e-6\right), \mathrm{q}^{*} \approx 1.2$ which gives $l^{*} \approx 5.2$. In the repulsion-dominated regime (Figure $3.18(\mathrm{~b})$ ), where the strain rate is relatively large, $\mathrm{S}^{*}$ disappears. Fast shearing prevents the cluster formation, leading to the homogeneous flow. 


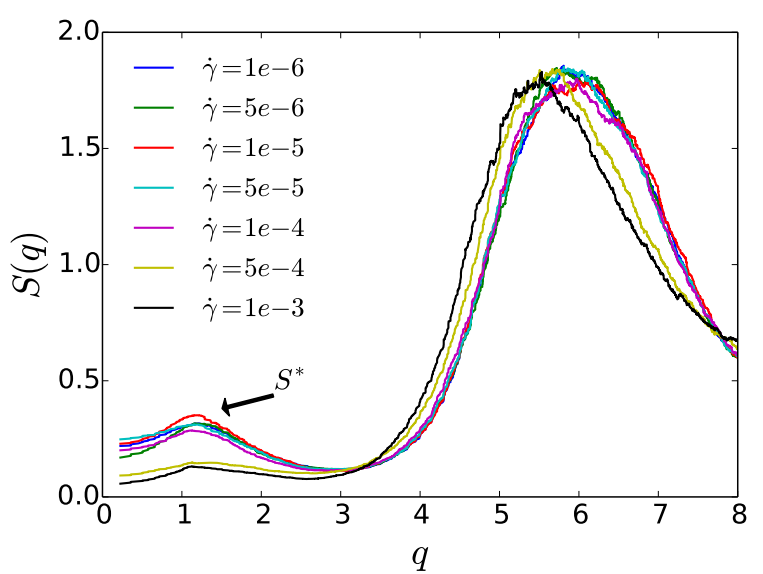

Figure 3.17: Structure factor $S(q)$ for different $\dot{\gamma}$ at $u=2 \times 10^{-4}$ and $\phi=0.75$. There is a small peak $S^{*}$ for $q<2$ associated with the local structure and particle clusters in the attraction-dominated regime. Since data points were noisy according to the small system size, they have been smoothed using the Savitzky-Golay filter.

In Figure 3.19 the attractive time-scale $\tau_{a}$ is used to rescale $\dot{\gamma}$ which collapses all $S^{*}$ data points for different attraction ranges on a single curve. It demonstrates the distinct effect of attractive interactions in determining the micro-structure over large length-scales.

For such a binary mixture that we study, one can further investigate the partial structure factors (Figure 3.20). They are defined as the following:

$$
\begin{aligned}
& S^{00}(q)=\frac{1}{N_{s}} \sum_{i, j}^{N_{s}} e^{i q\left(r_{i}-r_{j}\right)}, \\
& S^{11}(q)=\frac{1}{N_{l}} \sum_{i, j}^{N_{l}} e^{i q\left(r_{i}-r_{j}\right)}, \\
& S^{01}(q)=\frac{2}{N} \sum_{i}^{N_{s}} \sum_{j}^{N_{l}} e^{i q\left(r_{i}-r_{j}\right)},
\end{aligned}
$$

where $N_{s}$ and $N_{l}$ are the number of small and large particles $\left(N_{s}=\right.$ $\left.\mathrm{N}_{\mathrm{l}}=\mathrm{N} / 2\right) . S^{00}(\mathrm{q})$ corresponds to the smaller particle type with diameter $d=1.0, S^{11}(q)$ is calculated for the larger one with $d=1.4$ and $S^{01}(q)$ explores the structures only made of two different types of particles. Therefore the total structure factor can be written as:

$$
S(q)=\frac{1}{2}\left[S^{00}(q)+S^{11}(q)\right]+S^{01}(q)
$$

Figure 3.20 shows these different partial structure factors for a system similar to Figure 3.18 (a), deep in the attraction-dominated regime. The first peak in $S_{00}(q)$ is much larger than the one in $S_{11}$. 
(a)

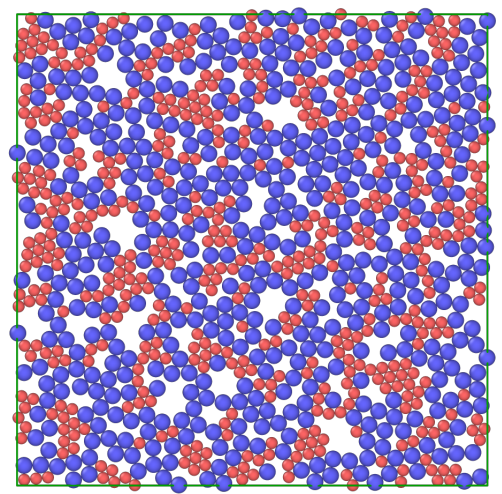

$(b)$

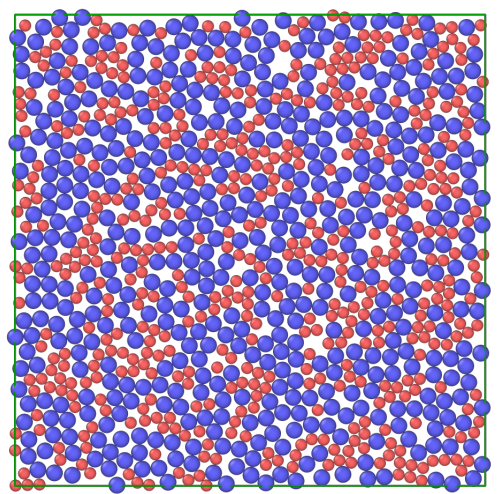

Figure 3.18: Two snapshots of the system in the steady state at $\phi=0.75$ and $u=2 \times 10^{-4}:(a)$, in the attraction-dominated regime, where $\dot{\gamma}=1 e-6$ and $(\mathbf{b})$, in the repulsion-dominated regime, where $\dot{\gamma}=1 e-3$. While the local structure and small clusters of particles, as well as voids, appear in the attraction-dominated regime, the system in the repulsion-dominated regime is homogeneous.

Thus, smaller particles contribute largely in the formation of clusters at attraction-dominated regime. This is a reasonable observation due to the fact that smaller particles have effectively more space than larger particles to form clusters.

It is tempting to attribute this effect to some sort of phase separation under shear. However, we did not observe any substantial growth of these meso-clusters on the time-scales accessible to our simulations. In Chapter 5, we will discuss that thermal fluctuations are necessary to evolve the system toward phase separation.

\subsection{PARTICLES DYNAMICS}

In the previous section we reported how weak and short-ranged attractive forces lead to the yield behavior, originating from the development of an isostatic structure. Other than the static structure of particle clusters, their dynamics is also affected by attraction. Displacements and velocity fluctuations are natural choices to study particles dynamics.

\subsubsection{Mean-squared Displacement}

To study particles trajectories, we investigated particles non-affine displacements, which is defined as particle displacement when the affine 


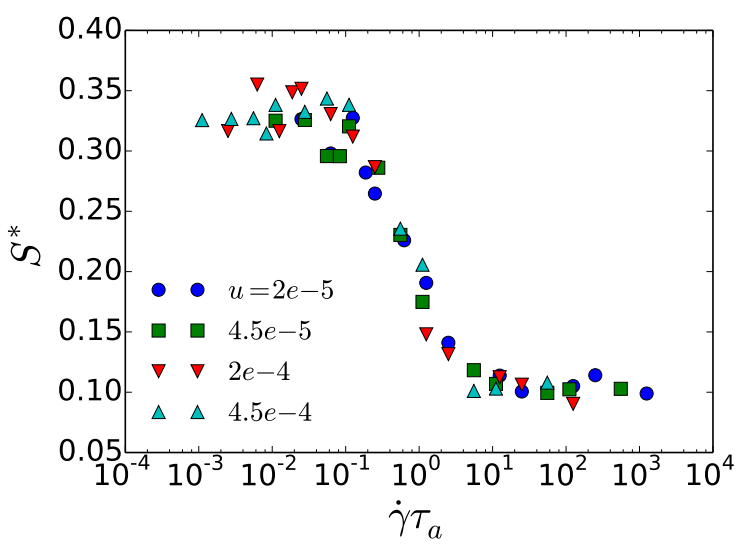

Figure 3.19: The first maximum in the structure factor, $\mathrm{S}^{*}$ as a function of $\dot{\gamma} \tau_{\mathrm{a}}$ for a system at $\phi=0.75$ and different attraction strength. The attractive timescale $\tau_{\mathrm{a}}$ is used to rescale the strain rate $\dot{\gamma}$.

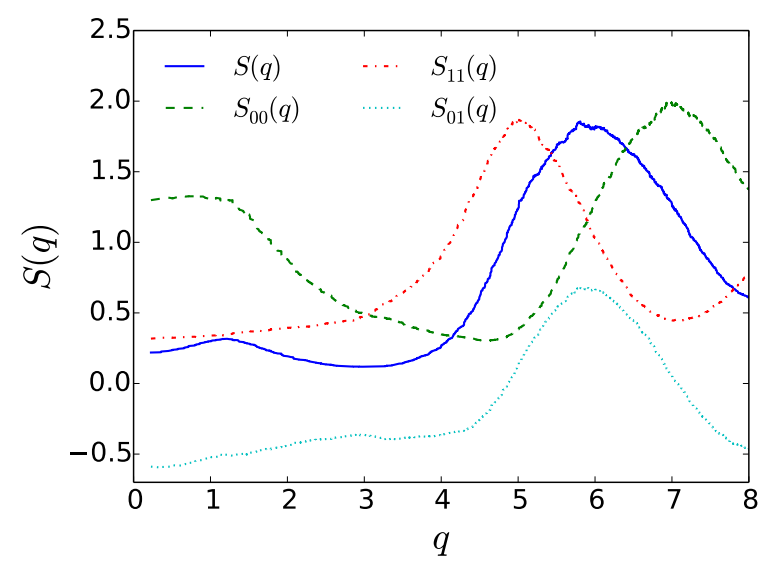

Figure 3.20: Partial structure factor $S(q)$ for a system at $u=2 \times 10^{-4}, \phi=$ 0.75 and $\dot{\gamma}=1 e-6$. S 00 corresponds to the smaller particles, while $S_{11}(q)$ measures the structure factor of larger particles.

part imposed by the shear flow is eliminated. At any time $t$, the nonaffine position of a particle can be written as:

$$
\mathbf{r}_{\text {naff. }}(\mathrm{t})=\mathbf{r}(\mathrm{t})-\hat{\mathbf{x}} \int_{0}^{\mathrm{t}} \mathrm{y}\left(\mathrm{t}^{\prime}\right) \dot{\gamma} d \mathrm{t}^{\prime}
$$

where $\mathbf{r}(t)=x(t) \hat{\mathbf{x}}+y(t) \hat{\mathbf{y}}$ is the position of particle at time $t, \hat{x}$ is the normal vector in the shearing direction and $\hat{y}$ is the normal vector in gradient direction. Then, mean-squared displacement in a desired direction is defined as

$$
\left\langle\operatorname{dr}(t)^{2}\right\rangle=\left\langle\left[r_{\text {naff. }}\left(t_{0}+t\right)-r_{\text {naff. }}\left(t_{0}\right)\right]^{2}\right\rangle
$$

where $r$ can be either $x$ or $y$, corresponding to the shearing and gradient directions.

Figure 3.22 shows the non-affine mean-squared displacement of particles at $u=2 \times 10^{-5}$ and $\phi=0.75$ for different strain rates. Since 


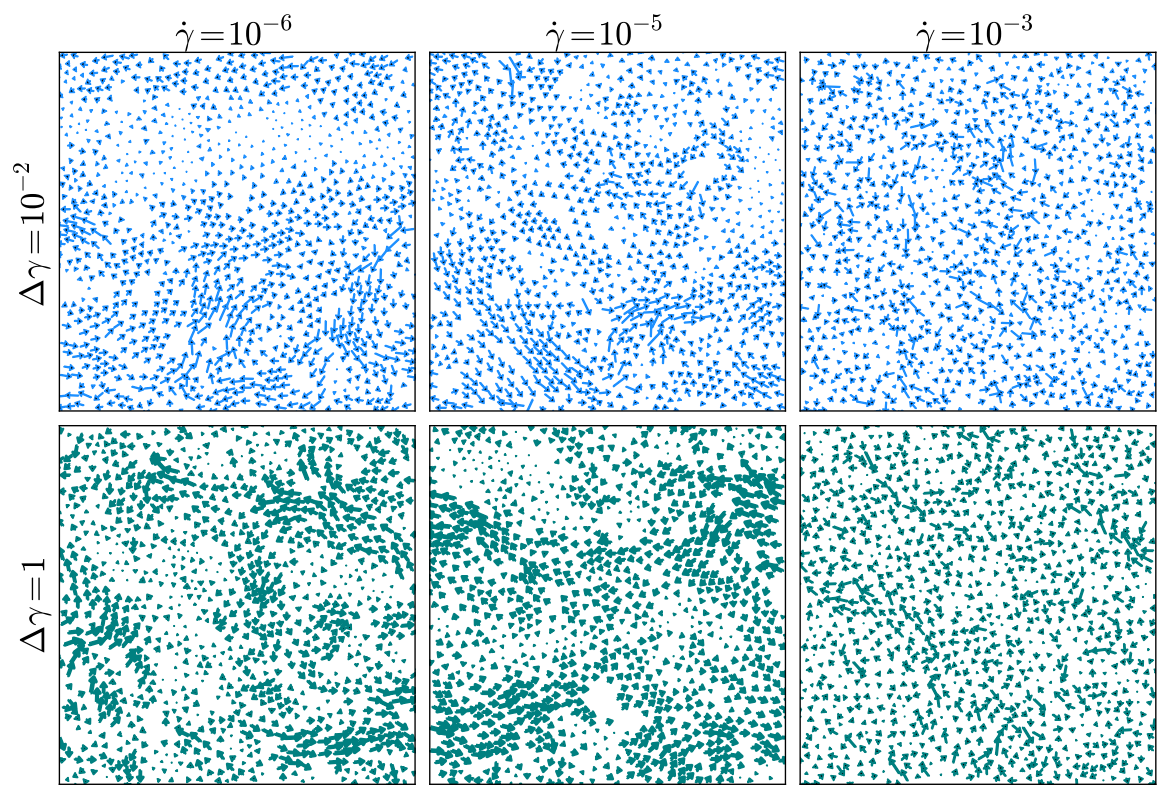

Figure 3.21: Displacement fields for a system at $u=2 \times 10^{-4}, \phi=0.75$ and different $\dot{\gamma}$. In upper panels, displacements are measured over strain window $\Delta \gamma=10^{-2}$, while in lower panels the strain window is $\Delta \gamma=1$.

in our simulations strain $\gamma$ is linearly proportional to the time, $\gamma=\dot{\gamma} t$, we report $\left\langle\mathrm{dr}^{2}\right\rangle$ as a function of $\gamma$, instead of time. In the repulsiondominated regime, there is no difference between the MSD in shearing and in gradient direction [see Fig. 3.22(d)].

Deep in the attraction-dominated and repulsion-dominated regimes, diffusive behavior is observed for large strains [Fig. 3.22(a) and (d)]. In the attraction-dominated regime, particles are attached to the isostatic network of particles and in case of losing contacts and leaving its location, they reconstruct contacts soon. It makes the time to observe diffusion much longer than the one in a repulsive system. Anisotropic behavoiur of the flow is also revealed by non-affine MSD calculation in the intermediate regime that is associated with large fluctuations of velocity and displacement in shearing direction (Fig. 3.22 (c)). These enhanced fluctuations, lead to the shear band formation in large enough systems, which we will discuss in the next section. In contrast to the gradient direction, particles hardly show diffusive behavior in the shearing direction over the simulation time we used. The anisotropic flow is more pronounced around the minimum of the flow curve, where the attractive branch meets the repulsive one.

Our results show that the minimum in non-monotonic flow curve, the maximum in the shear-stress ratio and the enhanced anisotropic behavior, are all associated to an almost same range of strain rates. The following scenario justifies these observations. In the limit of $\dot{\gamma} \rightarrow 0$ the nearly isostatic and system spanning structure forms due 


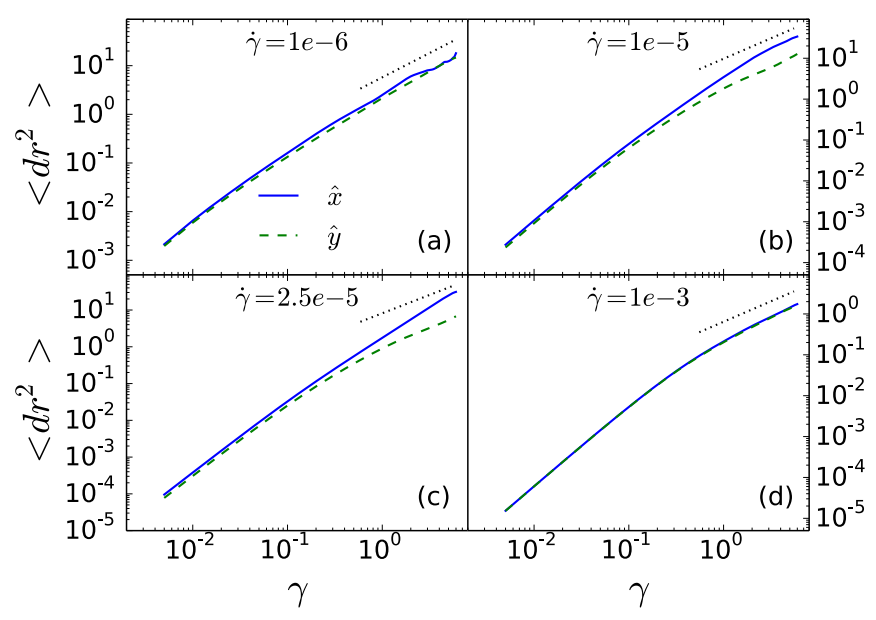

Figure 3.22: non-affine mean-squared displacement for (a) where the system is close to yield regime, (b) and (c) around the minimum in the flow curve where the attractive and repulsive branches meet and (d) in the repulsion-dominated regime for a system at $u=$ $2 \times 10^{-5}$ and $\phi=0.75$.

to the short relaxation time compared to the shearing time scale. It resists to flow and its structure is reconstructed shortly after the shear induced rupture. Increasing the strain rate leads to stronger resistance of the structure (increasing $\mu$ ) and also fluctuations in the shearing direction (anisotropy). At a certain strain rate $\left(1 / \tau_{a}\right)$, the attractive aggregation fails to compete with the shear-induced destruction, resulting the decrease of $\mu$ and the drop of $z$ below $z_{\text {iso }}$ with $\dot{\gamma}$. Since there is no more local particle clusters to impose large fluctuations in the shearing direction, the system behaves as an isotropic flow again.

In the diffusive regime, the mean-squared displacement is proportional to the diffusion constant $\mathrm{D}$ as:

$$
\left\langle\left[x\left(t_{0}+t\right)-x\left(t_{0}\right)\right]^{2}\right\rangle=2 D t .
$$

Therefore, fitting a linear function to the mean-squared displacement at large strains, gives the diffusion constant of the system. In Figure 3.23 , the diffusion constant in the gradient direction, $\mathrm{D}_{y}$ is plotted as a function of $\dot{\gamma} \tau_{\mathrm{a}}$ for a system at $\phi=0.75$ and different attraction ranges. Larger values of $D_{y}$ in low strain rates indicates that in contrast to the repulsion-dominated regime, non-affine displacements dominate the diffusion of the particles in the attraction-dominated regime at large timescale. 


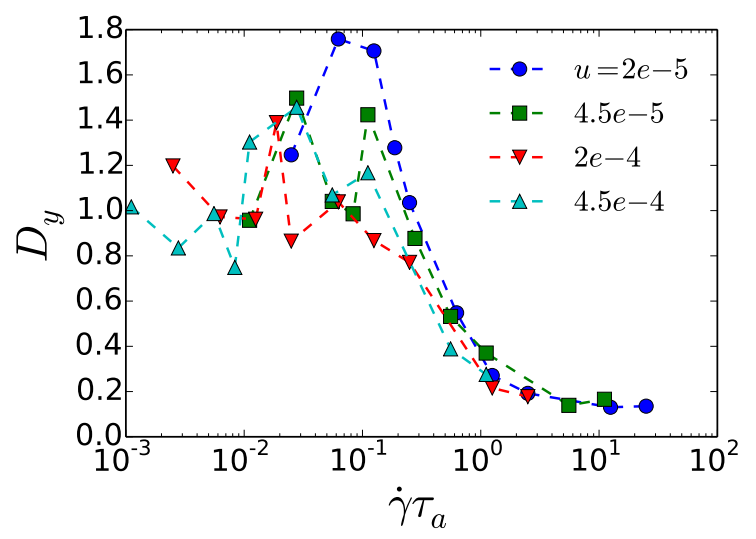

Figure 3.23: Diffusion constant in gradient direction, $D_{y}$ as a function of rescaled strain rate, $\dot{\gamma} \tau_{a}$, for a system at $\phi=2 e-5$ and different u.

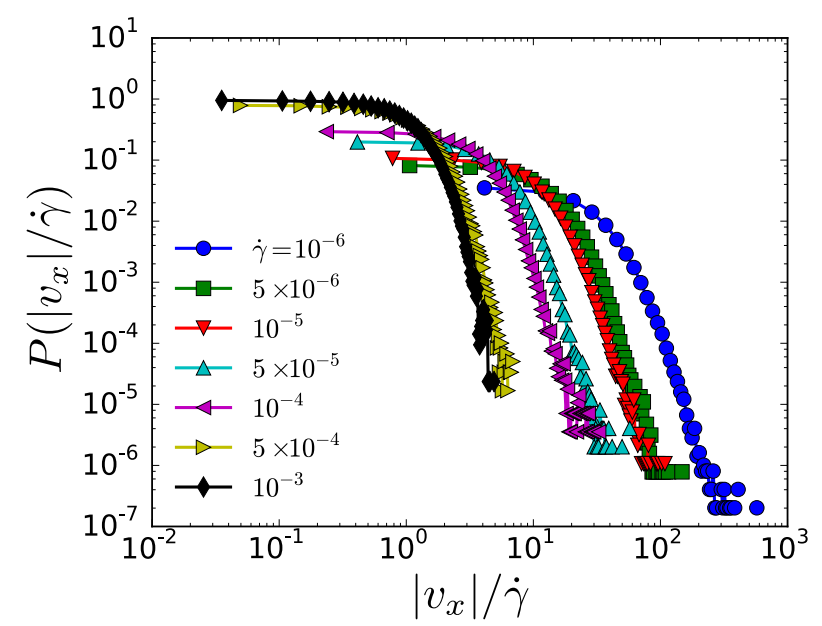

Figure 3.24: Probability distributions of non-affine velocity in the shearing direction, for a system at $u=2 \times 10^{-4}, \phi=0.75$ and different $\dot{\gamma}$

\subsubsection{Velocity Fluctuations}

While the diffusion constant measures displacement fluctuations in large strains, velocity fluctuations capture particles' dynamics in small strains (short timescales). The mean-squared velocity is defined as

$$
\left\langle(v(\mathrm{t}) / \dot{\gamma})^{2}\right\rangle=\left\langle\left[\left(v\left(\mathrm{t}_{0}+\mathrm{t}\right)-v\left(\mathrm{t}_{0}\right)\right) / \dot{\gamma}\right]^{2}\right\rangle .
$$

To eliminate the role of the strain rate, velocities are devided by $\dot{\gamma}$. Since in short times $\mathrm{dr} \sim v \Delta \mathrm{t}$, thus $\left\langle(v(\mathrm{t}) / \dot{\gamma})^{2}\right\rangle$ measures the slope of $\left\langle d r^{2}\right\rangle$ in short times or in the ballistic regime. Figure 3.25 displays that $\left\langle(v(t) / \dot{\gamma})^{2}\right\rangle$ can be collapsed on a single master curve if the strain rate is rescaled by the attractive time-scale. We found that the dependence of $v^{2}$ on $\dot{\gamma}$ in our results is consistent with [Ono et al., 2003]. 
In the context of non-affine motion, both of diffusion constant and mean-squared velocity, exhibit faster motion of particles in the attractiondominated regime. These results can be understood by noting the fact that attractive contacts in the attraction dominated regime, make affine displacements more costly (in terms of energy) than the nonaffine displacements. So non-affine displacements are dominant at small strain rates. As discussed previously, particles hardly diffuse in our simulation time at some range of strain rates, specially in the shearing direction. Therefore the corresponding data points in Figure 3.23 are the lower bound of the diffusion constant D.

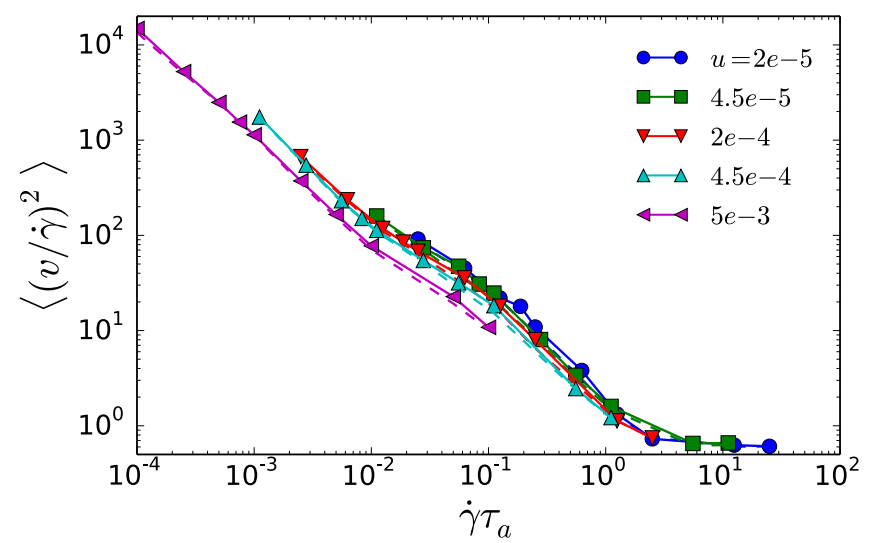

Figure 3.25: Variation of non-affine mean-squared velocity and diffusion constant with strain rate (scaled by the attractive time-scale) in shearing and gradient directions for a system at $\phi=0.75$. While solid lines represent the shearing direction, dashed lines correspond to the gradient direction.

\subsection{CONSitutive RELAtion: THE Fluidity MOdEL AP- PROACH}

It is necessary to build a theoretical model in order to support the physical explanation we provided for the flow behaviour of the system. To derive a relevant constitutive equation for the system we use the Fluidity model approach, which is discussed in [Olmsted, 2008].

We assume that the stress is composed of two separate contributions from elastic forces and the viscous term:

$$
\sigma=\eta \dot{\gamma}+\sigma_{\mathrm{s}}
$$

with $\eta$ indicating the viscosity of the system and $\sigma_{s}$ corresponding to the elastic stress. The rheological properties of the system is governed by the temporal evolution of $\sigma_{s}$ which is explained by an additional equation of motion. In our system, we take the viscosity to scale linearly with the strain rate $(\eta \sim \dot{\gamma})$, to obtain the Bagnold scaling in the repulsion-dominated regime. 
We define fluidity as reciprocal of viscosity, $a=1 / \eta$ and its evolution describes the elastic stress. Fluidity is a measure for the extent of aggregation into a network structure. In systems with high fluidity, only small aggregation occurs while a vanishing fluidity associates to a solid-like state. Among different choices for the evolution equation of the fluidity mentioned in [Picard et al., 2002], we take a simple exponential relaxation as

$$
\dot{\mathrm{a}}=\frac{-\mathrm{a}}{\tau_{0}}+\mathrm{r}_{1} \sigma_{\mathrm{s}} \dot{\gamma}
$$

with a relaxation rate

$$
1 / \tau_{0}=e^{-r_{0} \sigma_{s} \dot{\gamma}}
$$

The term $r_{1} \sigma_{s} \dot{\gamma}$ reflects the shear induced fluidization and it is balanced through a relaxation process explained by the term $-a / \tau_{0}$. As Equation 3.36 indicates, the relaxation rate itself also depends on the shear in such a way that it gets inhibited by shear in a less-fluid state. It is warranted in our simulations, since the local energy input $\sigma_{s} \dot{\gamma}$ gets dissipated via inelastic collisions, before a rigid structure forms.

Assuming the steady state condition, one can solve the equations above and obtain

$$
\sigma_{s}=\sigma_{y} \frac{W(\dot{\gamma} \tau)}{\dot{\gamma} \tau}
$$

with $W(x)$ being the Lambert-W function, $\sigma_{y}=1 / \sqrt{r_{1}}$ the yield stress, and the time-scale $\tau=r_{0} / 2 \sqrt{r_{1}}$. Therefore, the total stress can be written as

$$
\sigma(\dot{\gamma})=\sigma_{y}^{(\text {att })} \frac{W(\dot{\gamma} \tau)}{\dot{\gamma} \tau}+\sigma^{(\text {rep })}(\dot{\gamma})
$$

where the time-scale is taken to depends on the inverse attraction strength, $\tau \sim 1 / \mathrm{u}$, as we found earlier as the attractive time-scale $\tau_{\mathrm{a}}$. The stress in the repulsion-dominated regime is assumed to show the Bagnold scaling. Since

$$
\sigma^{(\text {rep })}(\dot{\gamma})=\eta \dot{\gamma}
$$

we conclude that $\eta \sim \dot{\gamma}$ which governs the Bagnold scaling.

Using the constitutive model given by Equation 3.38, we obtain the flow-curves presented by dashed lines in Figure 3.26. The nonmonotonic and the Bagnold scaling are well fitted by the constitutive equation.

In the limit of fast shearing $\left(\dot{\gamma} \gtrsim 10^{-3}\right.$, although the precise threshold depends on $u$ and $\phi$ ), soft-core effects appear in the system. Particles are energetic enough to pass through each-other, leading to a deviation from the Bagnold scaling. The model can not reproduce 

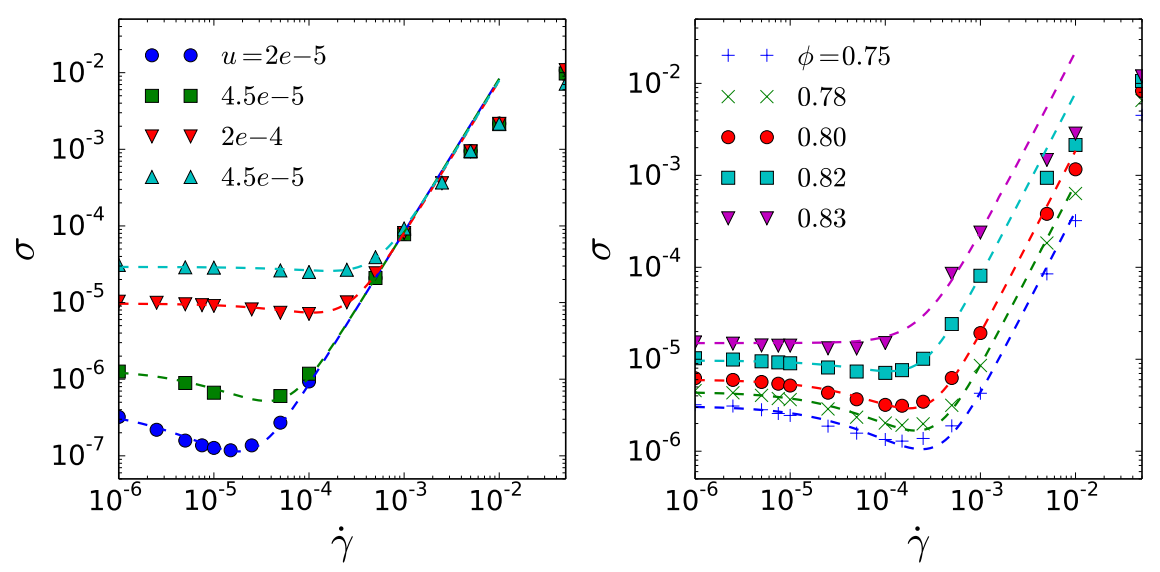

Figure 3.26: Non-monotonic flow-curves for systems at (left) $\phi=0.82$ but different attraction and (right) $u=2 \times 10^{-4}$ but different volume fractions. Dashed lines indicates the fitted function derived from the constitutive equation 3.38 .

some other details of the simulation results. For example, in the limit of small $\dot{\gamma}$, the fluidity model predicts $\sigma \rightarrow \sigma_{y} /\left(1+\dot{\gamma} \tau_{a}\right)$ while the simulation data reveals a weaker dependence like a logarithmic function.

\section{II SHEAR BANDS: THEORY}

It has been mentioned in the Introduction chapter that the non-monotonic $\sigma(\dot{\gamma})$ is a signature of mechanical instability [Fielding, 2014; Ovarlez et al., 2009; Picard et al., 2002; Schall and Hecke, 2010]. Therefore it is reasonable to expect shear-banding in systems with such strong nonmonotonic flow-curves presented in Figure 3.3. However, no shear bands are observed for corresponding systems with $N=1000$. To know why, we need to have a theoretical review on the concept of shear-banding.

\subsubsection{Constitutive Equation}

In a two-dimensional flow subject to simple shearing, the linear velocity profile governs. Thus, the fluid velocity $\mathbf{v}$ is along the shearing direction $\hat{x}$ and it is a function of the distance from the bottom of the cell, $y$. For such a system the Navier-Stokes equation is written as

$$
\rho \frac{\partial v(y, t)}{\partial t}=\frac{\partial \Sigma(y, t)}{\partial y}
$$

where $\rho$ is the mass density and $\Sigma$ indicates the stress. The stress follows the constitutive relation,

$$
\Sigma(y, t)=\eta(\dot{\gamma}(y, t)) \dot{\gamma}(y, t)
$$


with $\dot{\gamma}(y, t)=\partial v(y, t) / \partial y$ as the local strain rate and $\eta$ as the viscosity. According to Equation 3.40, the stress should be constant in the stationary state which is equivalent to the mechanical stability.

In the shear-banded state, the system is divided in two bands with different strain rates, connecting with an interface. The stress obtained from the standard constitutive relation satisfies the mechanical stability condition in bands, but not in the interface where $\dot{\gamma}$ is not constant. Hence, following the work by Dhont [Dhont, 1999] one can conclude that the constitutive relation needs be revised.

To derive the standard constitutive relation (Equation 3.41) only first-order spatial derivatives of the flow velocity are considered to contribute to the stress. Regarding the shear banded state, this assumption is not valid any more and higher-order spatial derivatives should be also accounted for as well to include the spatial dependence of the viscosity, $\eta=\eta(y, t)$. The symmetry properties of $\Sigma$ indicates that the additional contribution should be in the form of $\sim \partial^{2} \dot{\gamma}(y, t) / \partial y^{2}$. The reason that this additional term is not considered in molecular systems is that the range of interactions is very small in such systems. However, larger size of particles in colloidal macromolecules or granular material, leads to a contribution of even relatively small gradients of strain rate, to the stress.

Now, the constitutive relation can be written as

$$
\Sigma(y, t)=\eta(\dot{\gamma}(y, t)) \dot{\gamma}(y, t)-\kappa(\dot{\gamma}(y, t)) \frac{\partial^{2} \dot{\gamma}(y, t)}{\partial y^{2}} .
$$

Parameter $\mathrm{K}$, the shear-curvature viscosity has been first introduced by Dhont [Dhont, 1999] and is called. A similar expression for the stress is also given by Olmsted [D. Olmsted, Peter and David Lu, CY., 1999]. Later we will derive the analytical expression for $\kappa=\kappa(\dot{\gamma})$ and connect it to the interface width between shear bands.

Now for the stationary state one can write

$$
\Sigma_{\text {stat. }}=\eta(\dot{\gamma}(y)) \dot{\gamma}(y)-\kappa(\dot{\gamma}(y)) \frac{d^{2} \dot{\gamma}(y)}{d y^{2}}
$$

where the time dependence is gone due to the stationary flow conditions and the stress is constant all over the system, $\Sigma(y)=\Sigma_{\text {stat. }}$.

\subsubsection{Van der Waals Loop}

Consider a shear-banded state of an example system like Figure 3.28 (a) and (b). There are two bands with different strain rates $\left(\dot{\gamma}_{-}\right.$and $\dot{\gamma}_{+}$) connecting with an interface with width size $w$. To calculate the stress contribution of the term $\eta(\dot{\gamma}(y)) \dot{\gamma}(y)$, it is necessary to obtain the strain rate dependence of the viscosity. Since viscosity is an even 


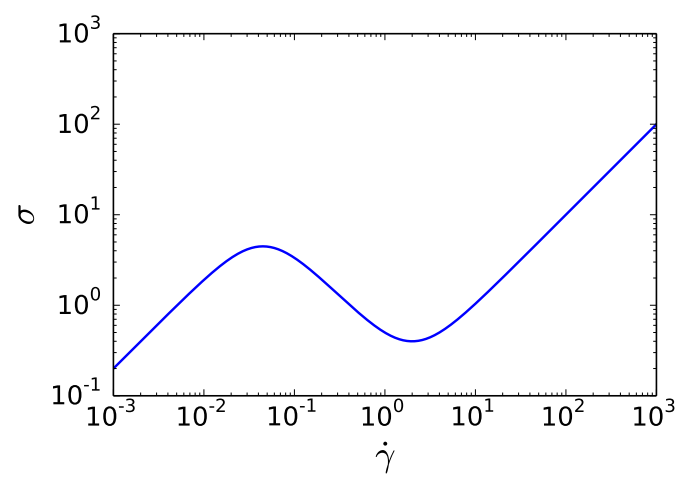

Figure 3.27: the stress contribution of $\sigma=\eta(\dot{\gamma}) \dot{\gamma}$ as a function of strain rate $\dot{\gamma}$, for a simple model described in Equation 3.44. To obtain $\eta(\dot{\gamma})$, we set $\eta_{0}=200, \eta_{\infty}=0.1$ and $\alpha=500$.

function of the strain rate, $\eta(-\dot{\gamma})=\eta(\dot{\gamma})$, we choose the simplest form of $\eta(\dot{\gamma})$ which also shows a van der Waals-like behavior:

$$
\eta(\dot{\gamma})=\frac{\eta_{0}+\alpha \eta_{\infty} \dot{\gamma}^{2}}{1+\alpha \dot{\gamma}^{2}}
$$

where zero and large limits of the strain rate are indicated by $\eta_{0}$ and $\eta_{\infty}$ respectively. The parameter $\alpha$ can be chosen to find the best fit to the experimental data, which is not important for the purpose of theoretical discussion here. Figure 3.27 displays the resulting flow curve $\sigma=\eta(\dot{\gamma}) \dot{\gamma}$, exhibiting a non-monotonic behavior.

Figure 3.28 represents a typical shear-banded state of the system. In panel (a), the velocity profile of the system displays two bands: one at the top of the system with almost zero flow rate, and the other flowing with a local strain rate much larger than the imposed strain rate, $\dot{\gamma}_{0}$. Panel (b), displays the local strain rate profile of the system, which is basically the derivative of the velocity profile presented in panel (a) with respect to $y, \dot{\gamma}(y)=d v_{x}(y) / d y$.

Crossing the interface, the term $\sigma(\dot{\gamma})=\eta(\dot{\gamma}(y)) \dot{\gamma}(y)$ contributes to the stress with a large gradient following the van der Waals-like behavior, as is depicted in Figure 3.28(c).

Panel (d) in Figure 3.28 illustrates the variation of $\mathrm{d}^{2} \dot{\gamma} / \mathrm{dy} \mathrm{y}^{2}$ with respect to $y$, showing the same symmetry as $\sigma(y)$. To obtain a uniform stress over the whole system in the stationary state, the change of $\sigma(y)$ should be compensated for with the stress contribution from the curvature-viscosity term.

Including the shear-curvature viscosity contribution, the NavierStokes equation can be rewritten as

$$
\begin{aligned}
\rho \frac{\partial v(y, t)}{\partial t}= & \frac{d \sigma(\dot{\gamma}(y, t))}{d y} \frac{\partial \dot{\gamma}(y, t)}{\partial y}-\kappa(\dot{\gamma}(y, t)) \frac{\partial^{3} \dot{\gamma}(y, t)}{\partial y^{3}} \\
& -\frac{d \kappa(\dot{\gamma}(y, t))}{d \dot{\gamma}(y, t)} \frac{\partial \dot{\gamma}(y, t)}{\partial y} \frac{\partial^{2} \dot{\gamma}(y, t)}{\partial y^{2}}
\end{aligned}
$$



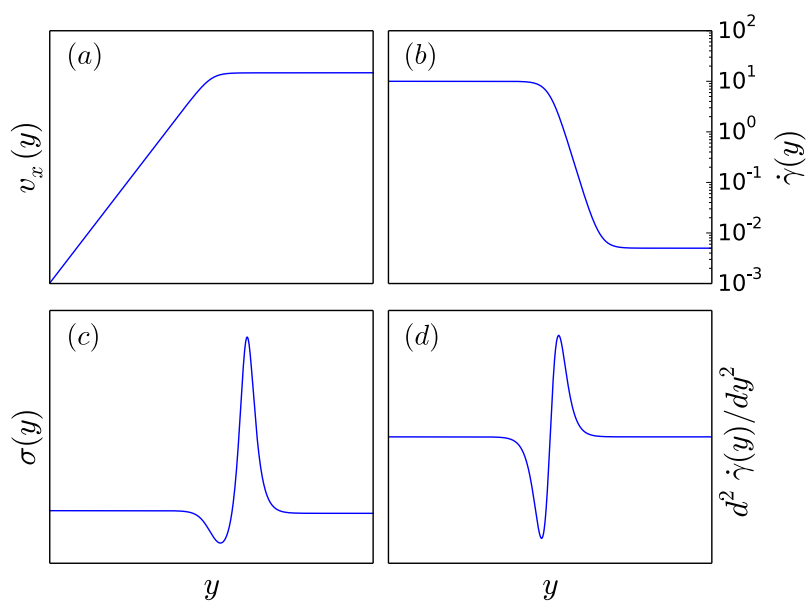

Figure 3.28: (a) The velocity profile of a shear banded system. (b) The strain rate profile of the same system as (a). (c) the variation of $\sigma=$ $\eta(\dot{\gamma}) \dot{\gamma}$ for the same system as (a). (d), the second derivative of the local strain rate, $d^{2} \dot{\gamma}(y) / d y^{2}$ as a function of $y$ for the same system as (a).

In the case we are interested in, the plane shear geometry, boundary conditions are read as

$$
\begin{aligned}
v(\mathrm{y}=0, \mathrm{t})) & =0, \\
v(\mathrm{y}=\mathrm{L}, \mathrm{t}) & =\dot{\gamma}_{\mathrm{o}} \mathrm{L},
\end{aligned}
$$

where $\mathrm{L}$ is the size of the system in the gradient direction and $\dot{\gamma}$ is the external strain rate.

\subsubsection{Maxwell Construction and Phase Coexistence}

Consider a shear-banded state of the system like Figure 3.28 (a) and (b) where two bands with different strain rates $\left(\dot{\gamma}_{-}\right.$and $\left.\dot{\gamma}_{+}\right)$are connected with an interface with width size $w$. In the stationary state, the integration of Equation 3.43 from $\dot{\gamma}_{-}$to $\dot{\gamma}_{+}$reads as

$$
\int_{\dot{\gamma}_{-}}^{\dot{\gamma}_{+}} \frac{\sigma(\dot{\gamma})-\Sigma_{\text {stat. }}}{\kappa(\dot{\gamma})} \dot{d}=0
$$

If $\mathrm{k}$ is assumed to be independent from the strain rate $\dot{\gamma}$, Equation 3.47 reduces to

$$
\Sigma_{\text {stat. }}=\left(\dot{\gamma}_{+}-\dot{\gamma}_{-}\right)^{-1} \int_{\dot{\gamma}_{-}}^{\dot{\gamma}_{+}} \mathrm{d} \dot{\gamma} \sigma(\dot{\gamma})
$$

which implies the stress selection by a Maxwell equal area construction in the $(\sigma, \dot{\gamma})$ plane. Maxwell equal area construction results in the replacement of the unstable part of the flow-curve by a plateau, along which shear bands coexist. 


\subsubsection{Linear Stability Analysis: Size Matters}

In this section, we analyze the stability of linear velocity profiles in non-banded systems, using linear stability tools. Assuming that $\delta v(y, t)$ indicates a small deviation from a linear velocity profile due to the imposed strain rate, $\dot{\gamma}_{0}$. After linearizing Equation 3.45 with respect to the small perturbation, it can be written as

$$
\rho \frac{\partial \delta v(y, t)}{\partial t}=\frac{d \sigma\left(\dot{\gamma}_{0}\right)}{d \dot{\gamma}_{0}} \frac{\partial^{2} \delta v(y, t)}{\partial y^{2}}-\kappa\left(\dot{\gamma}_{0}\right) \frac{\partial^{4} \delta v(y, t)}{\partial y^{4}} .
$$

The solution of this equation can be given in terms of the Fourier series. To impose the boundary condition $\delta v(y=0, t)=0$, we extend the solution to the interval $-\mathrm{L}<\mathrm{y}<\mathrm{L}$ and use the sine-series expansion only

$$
\delta v(y, t)=\sum_{n=1}^{\infty} a_{n}(t) \sin \left(k_{n} y\right)
$$

where the wave numbers $k_{n}$ are defined as

$$
k_{n}=\frac{\pi n}{L}, \quad n=1,2,3, \ldots .
$$

Now, by substituting Equation 3.50 in Equation 3.49, it can be written as

$\rho \sum_{n=1}^{\infty} \frac{d a_{n}(t)}{d t} \sin \left(k_{n} y\right)=\sum_{n=1}^{\infty} a_{n}(t) \sin \left(k_{n} y\right)\left[-k_{n}^{2}\left(\frac{d \sigma_{0}\left(\dot{\gamma}_{0}\right)}{d \dot{\gamma}_{0}}+k\left(\dot{\gamma}_{0}\right) k_{n}^{2}\right)\right]$.

Therefore, the time evolution of Fourier coefficients are given as

$$
a_{n}(t)=a_{n} \exp \left(-\left[\frac{d \sigma_{0}\left(\dot{\gamma}_{0}\right)}{d \dot{\gamma}_{0}}+\kappa\left(\dot{\gamma}_{0}\right) k_{n}^{2}\right] \frac{k_{n}^{2} t}{\rho}\right),
$$

with $a_{n} \equiv a_{n}(t=0)$ indicating the initial perturbation. Therefore, deviation from the linear velocity profile can be written as

$$
\delta v(y, t)=\sum_{n=1}^{\infty} a_{n} \sin \left(k_{n} y\right) \times \exp \left(-\left[\frac{d \sigma_{0}\left(\dot{\gamma}_{0}\right)}{d \dot{\gamma}_{0}}+\kappa\left(\dot{\gamma}_{0}\right) k_{n}^{2}\right] \frac{k_{n}^{2} t}{\rho}\right)
$$

The exponential dependence of $\delta v$ on $t$ determines the proper condition for a stable velocity profile. In the case of imposed strain rate, $\dot{\gamma}_{0}$, the velocity profile is unstable when

$$
\frac{\mathrm{d} \sigma\left(\dot{\gamma}_{0}\right)}{\mathrm{d} \dot{\gamma}_{0}}<-\kappa\left(\dot{\gamma}_{0}\right) \frac{\pi^{2}}{\mathrm{~L}^{2}}<0
$$


Then, the critical wave number can be define as

$$
k^{*}=\sqrt{-\frac{d \sigma\left(\dot{\gamma}_{0}\right) / d \dot{\gamma}_{0}}{\kappa(\dot{\gamma})}},
$$

where all Fourier modes with a wave number less than $k^{*}$ grow exponentially in time and dominate. Thus, for a stable velocity profile, all relevant wave number should be larger than $k^{*}$ which results in the exponential decay in amplitude of corresponding Fourier terms. It is necessary to note that here $\sigma(\dot{\gamma})$ is the total stress in the system before the shear-banding, where the velocity profile is still linear. As it is shown later, in the banded system the negative slope of $\sigma$ versus $\dot{\gamma}$ is less and in the thermodynamic limit, it approaches zero corresponding to the plateau determined by Maxwell area construction.

\subsection{SHEAR BANDS: SIMULATIONS}

\subsubsection{The form of $\mathrm{\kappa}(\dot{\gamma})$}

The theoretical discussion above reflects the importance of $k$ as the shear-curvature viscosity, in shear-banding phenomena. Now one can explain why no shear band forms in the system presented in Figure 3.30 with $N=1000$. The shear band formation can not be observed if the wavelengths of unstable modes do not fit inside the system. Hence, to observe the shear localization it is necessary to have a large enough system [Dhont, 1999].

The fluidity model presented in Section 3.10 provides an analytical expression for non-monotonic flow curves, rewritten as

$$
\sigma(\dot{\gamma})=\sigma_{y}^{(\mathrm{att})} \frac{W(\dot{\gamma} \tau)}{\dot{\gamma} \tau}+\beta \dot{\gamma}^{2}
$$

with $\beta=\sigma^{(\text {rep })} / \dot{\gamma}^{2}$. Making a derivative with respect to $\dot{\gamma}$, we reach

$$
\sigma^{\prime}(\dot{\gamma})=\frac{\mathrm{d} \sigma(\dot{\gamma})}{\mathrm{d} \dot{\gamma}}=-\sigma_{y} \frac{W^{2}(\tau \dot{\gamma})}{\tau \dot{\gamma}^{2}(W(\tau \dot{\gamma})+1)}+2 \beta \dot{\gamma}
$$

The critical system size, below which shear bands do not form, can be obtained from Equation 3.56,

$$
\mathrm{L}^{*}=\frac{2 \pi}{\mathrm{k}^{*}}=2 \pi \sqrt{-\frac{\mathrm{k}(\dot{\gamma})}{\sigma^{\prime}(\dot{\gamma})}} .
$$

Therefore, the shear-curvature viscosity can be written as

$$
\kappa(\dot{\gamma})=-\left(\frac{\mathrm{L}^{*}}{2 \pi}\right)^{2} \sigma^{\prime}(\dot{\gamma}),
$$

$\kappa$ has the same dependence on $\dot{\gamma}$ as $\sigma^{\prime}$. Furthermore, it is found to have

$$
\mathrm{K} \sim \mathrm{L}^{* 2} .
$$


The same scaling can also be found with respect to the interface width. For a shear-banded system in the stationary state, Equation 3.49 gives

$$
\frac{\mathrm{d} \sigma\left(\dot{\gamma}_{0}\right)}{\mathrm{d} \dot{\gamma}_{0}} \frac{\partial^{2} \delta v(\mathrm{y}, \mathrm{t})}{\partial \mathrm{y}^{2}}=\kappa\left(\dot{\gamma}_{0}\right) \frac{\partial^{4} \delta v(\mathrm{y}, \mathrm{t})}{\partial \mathrm{y}^{4}}
$$

in which integrating both sides over the interface width $\Delta w$, results in

$$
\mathrm{K} \sim \Delta w^{2} .
$$

The mentioned scaling relations can be checked by investigating shearbanding in systems at different attractions for instance.

\subsubsection{Flow Curves and System Size}

Increasing the system size from $N=1000$ to $N=10000$ quickly results in shear bands formation in the system. Figure 3.29 illustrates a typical non-affine velocity field of such a system $\left(\mathrm{N}=10^{4}\right.$, $u=2 \times 10^{-5}, \phi=0.82$ and $\dot{\gamma}=5 \times 10^{-6}$ ) with shear bands, and corresponding connectivity profile. The connectivity profile shows that one band forms as an isostatic network of particles and flows with almost zero strain rate, and the other one flows with a strain rate larger than the imposed one, as a fluid.

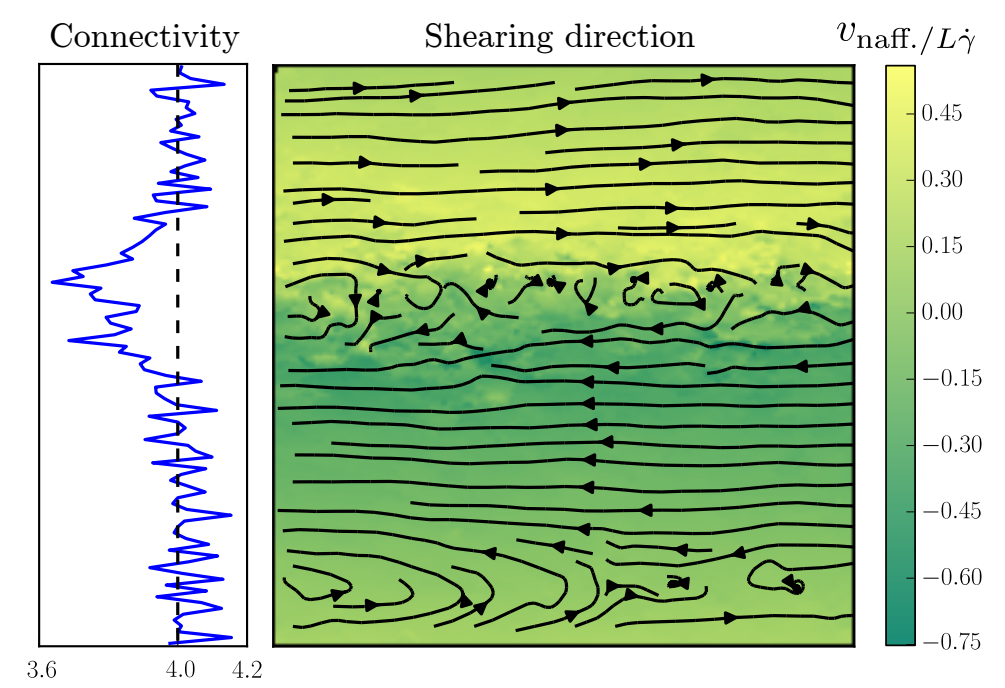

Figure 3.29: A snapshot of the non-affine velocity field in a system exhibiting shear-banding, using $\mathrm{N}=2 \times 10^{4}$ particles, $\phi=0.82, \mathrm{u}=2 \times$ $10^{-5}$ and $\dot{\gamma}=2.5 \times 10^{-6}$. The coloring is based on the velocity in the shearing direction. Stream lines show the non-affine flow field. The corresponding connectivity profile reveals that in the solid band, connectivity fluctuates around the isostactic value but decreases in the fludized band.

A valid question is how shear-banding affects the flow-curve. Figure 3.30 exhibits $\sigma(\dot{\gamma})$ for two different system sizes, with $N=1000$ 
and $\mathrm{N}=20000$. For $\mathrm{N}=20000$ we start shearing the system with a random initial configuration at $\dot{\gamma}=10^{-4}$, ramp it down until $\dot{\gamma}=$ $10^{-6}$ (circles) and then ramp it up (squares). At each $\dot{\gamma}$, the system is sheared for $\gamma=20$ except at $\dot{\gamma}=10^{-6}$ where the system is sheared for $\gamma=12$. Filled symbols indicate strain rates in which shear bands form. One can see that shear localization depresses the non-monotonic behavior of the flow curve, specifically in the ramp-up branch. The minimum of the flow curve is shallower in the ramp-up branch, while the range of strain rates in which the shear localization occurs is wider. Similar hysteresis effect is also observed in recent experiments using the same protocol [Divoux, Grenard, and Manneville, 2013]. As it has been suggested in several works, the stress-decreasing part of the flow curve is expected to be replaced by a straight line in the thermodynamic limit [Dhont, 1999; Ovarlez et al., 2009]. Our observations in simulations with different system sizes, are consistent with this trend.

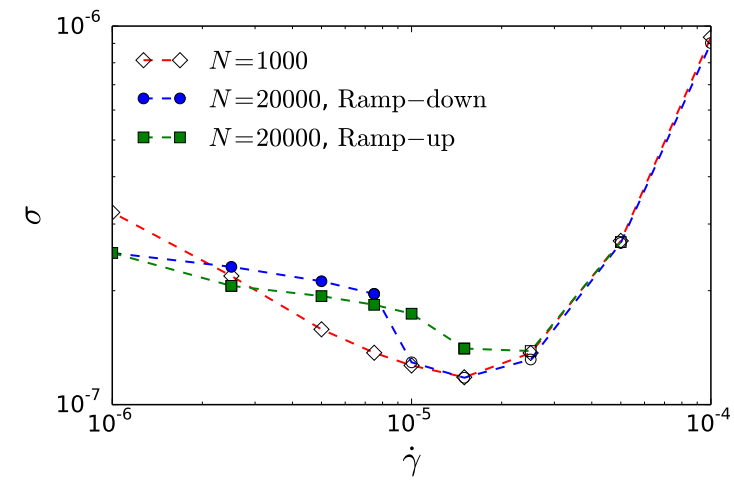

Figure 3.30: Flow curves for different system sizes $N$, at $\phi=0.82$ and $u=$ $2 \times 10^{-5}$. The non-monotonic part of the flow curve gets smaller as system size increases and the system is sheared longer. Filled symbols indicate shear band formation.

\subsubsection{Properties of Shear Bands}

We investigated how the spatial location of the fluidized band, in the shear gradient direction changes in time. In order to do that, we monitored the position of the center of the band. Figure 3.31 displays how that position changes in time as the imposed strain rates is ramped up. At small imposed $\dot{\gamma}$, the position of the band fluctuates, although the total strain $(\Delta \gamma=12$ or 20$)$ is not large enough for the band to traverse the entire system. As the strain rate increases and gets closer to the repulsion-dominated regime, the band moves more slowly which is consistent with our results for the non-affine motion of particles in small systems [see Fig. 3.23]. 


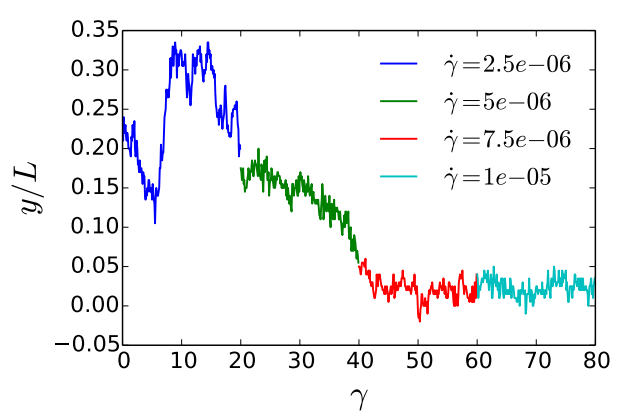

Figure 3.31: Position of the fludized band in time as the imposed $\dot{\gamma}$ is ramped up. Larger fluctuations at smaller $\dot{\gamma}$ is consistent with the results for the non-affine motion of particles in small systems.

To understand the effect of the applied strain rate, we also explore how the spatial profile of the shear-bands change, when the imposed strain rate is varied. In Figure 3.32 we show the spatial profile of local strain rates $\left(\dot{\gamma}_{\text {local }}\right)$, normalized by the imposed $\dot{\gamma}$. At the smallest strain rate, we have a localized band of large fluidization. Increasing the strain rate, we observe that the height of this spatial profile decreases, implying that the fluidized region has less contrast in flowrate with the solid-like region. Also, the width of this region broadens and the shear-band finally disappears in the regime where repulsion dominates. In all these cases, we have checked and found that the stress generated in the system is spatially uniform, albeit with minor fluctuations.

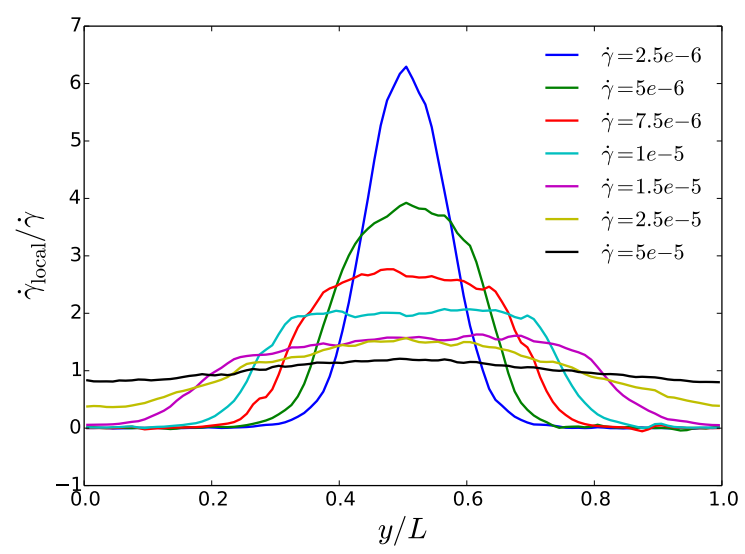

Figure 3.32: Spatial profiles of local strain rates, normalized by $\dot{\gamma}$, as it is ramped up. The contrast in the flow rates of bands decreases with increasing $\dot{\gamma}$.

From the spatial profiles of the shear-bands, we compute the width of the solid-like and fluid-like regions, as well as the interface of the shear-band (Figure 3.33). As described above, the width of the solidlike region decreases linearly and similarly the liquid-like region in- 
creases with increasing $\dot{\gamma}$. On the contrary, the width of the interface remains nearly constant, consistent with earlier numerical observations [Martens, Bocquet, and Barrat, 2012].

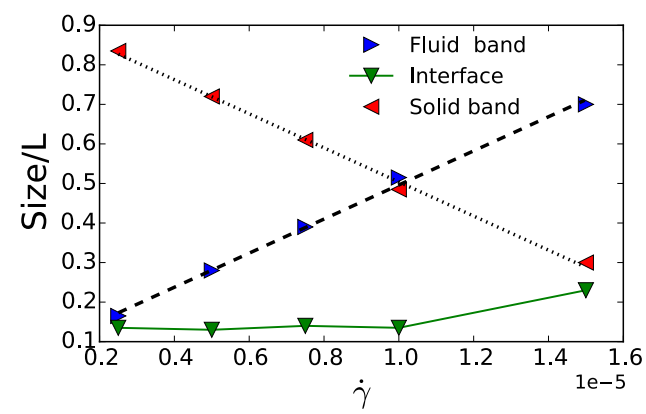

Figure 3.33: Shear bands and the interface width in units of system size, as a function of imposed strain rate $\dot{\gamma}$. The dashed line is the fitted linear function to the size of fluid band, $S_{f}(\dot{\gamma})=4.31 \times 10^{4} \times$ $\dot{\gamma}+6.51 \times 10^{-2}$. The dotted line presents the fitted function to the size of solid band which is by definition $S_{s}(\dot{\gamma})=1-S_{f}(\dot{\gamma})$. 


\section{THE ROLE OF DISSIPATION}

\subsection{INTRODUCTION}

In the previous chapter we presented how a weak attractive interaction changes the rheological response of soft particulate systems, close to the jamming point. We found non-monotonic flow-curves, rigidity below the jamming point and permanent shear-banding in large systems. In this chapter we address the question what is the role of dissipative forces in the context of attractive systems we study. The role of different models of energy dissipation on rheology of repulsive and frictionless systems is studied in [Vågberg et al., 2014]. They reported either Bagnoldian or Newtonian rheology depending on whether large clusters of particles develop in the system. They found tangential dissipation in collisions (over-damped regime), has a crucial role on the development of large clusters of repulsive particles.

The mass (inertia) is another important parameter to determine the mechanical response of the system. Reducing the damping eventually puts the system in the under-damped regime where the energy input due to shearing is dissipated in longer time, promoting the plastic flow. In the quasi-static limit, two different universality classes govern the statistics of avalanches in the over-damped and under-damped regimes [Salerno and Robbins, 2013; Salerno et al., 2012]. Weak dissipation in disordered solids might lead to heating up the system due to shearing, resulting in non-monotonic flow curves [Nicolas et al., 2016].

In this chapter we report our findings on the role of dissipation on rheology of attractive systems. we discuss two different models of contact dissipation. By varying the damping factor, we explore two flow regimes: the over-damped and the under-damped regime.

\subsection{DAMPING REGIMES}

Whenever two particles collide and form a contact, Equation 3.1 ensures that they can be modeled as a damped harmonic oscillator. Temporal evolution of such an oscillator in one dimension, is governed by the following differential equation:

$$
m \frac{d^{2} x}{d t^{2}}+b \frac{d x}{d t}+\epsilon x=0
$$


with $F_{c}=-\epsilon \chi$ and $F_{\text {diss }}=-b \frac{d x}{d t}$ are respectively conservative and damping forces. We can rewrite that equation as

$$
\frac{d^{2} x}{d t^{2}}+2 \beta \frac{d x}{d t}+\omega_{0}^{2} x=0
$$

where $\beta \equiv \mathrm{b} / 2 \mathrm{~m}$ is the damping ratio and $\omega_{0}^{2}=\sqrt{\epsilon / \mathrm{m}}$ is the natural angular frequency. This is a homogeneous second-order differential equation, with solution of the form

$$
x(t)=e^{\lambda t} .
$$

Substituting it in Equation 4.2, we reach the auxiliary equation for $\lambda$ as

$$
\lambda^{2}+2 \beta \lambda+\omega_{0}^{2}=0,
$$

which has two roots as

$$
\lambda=-\beta \pm \sqrt{\beta^{2}-\omega_{0}^{2}} .
$$

Therefore, the general solution of Equation 4.2 is given by

$$
x(t)=e^{\beta t}\left[A_{1} \exp \left(\sqrt{\beta^{2}-\omega_{0}^{2}} t\right)+A_{2} \exp \left(\sqrt{\beta^{2}-\omega_{0}^{2}} t\right)\right]
$$

The harmonic oscillator displays three different behavior, depending on the value of $\beta^{2}-\omega_{0}^{2}$. Those categories are called over-damped, under-damped and critical damping which are discussed in the following subsections.

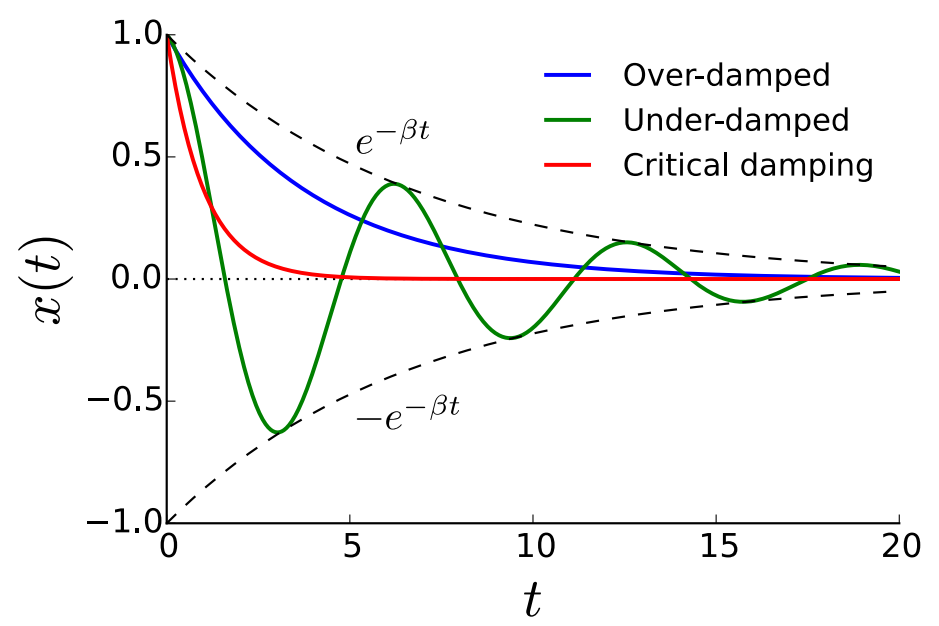

Figure 4.1: Time evolution of a damped harmonic oscillator, in three different damping regimes. Data point are calculated from Equation 4.6 , with $A_{1}=1, A_{2}=0$ and $\omega_{0}=1$. Damping factor $\beta$ is set to $\beta=2$ for over-damped, $\beta=0.15$ for under-damped and $\beta=1.0$ for critical damping. 


\subsubsection{Over-damped Oscillator}

If $\beta^{2}-\omega_{0}^{2}>0$, the oscillator is in the over-damped regime. In this case, Equation 4.6 becomes,

$$
x(t)=e^{-\beta t}\left[A_{1} e^{\omega_{1} t}+A_{2} e^{-\omega_{1} t}\right],
$$

where $\omega_{1}=\sqrt{\beta^{2}-\omega_{0}^{2}}$. In the over-damped regime, the decay term $\exp (\beta t)$ dominates, $\chi(t)$ does not oscillate and asymptotically reaches $x=0$. Such an over-damped $x(t)$ is plotted in Figure 4.1, in blue color. In the context of colliding particles in the over-damped regime, their relative velocity is dissipated very fast, hence particles stay next to each-other after the collision.

\subsubsection{Under-damped Oscillator}

If $\beta^{2}-\omega_{0}^{2}<0$, then the oscilator is in the under-damped regime. In this case, it is better to define a new frequency as

$$
\omega_{2} \equiv \omega_{0}^{2}-\beta^{2},
$$

which is called the damped oscillator frequency. Then, Equation 4.6 can be written as

$$
x(t)=e^{-\beta t}\left[A_{1} e^{i \omega_{2} t}+A_{2} e^{-i \omega_{2} t}\right] .
$$

However, $\omega_{2}$ is not a true frequency since the oscillator never returns to a point with the same velocity. The maximum of $x(t)$ decays in time due to the exponential decay of $e^{-\beta t}$. Figure 4.I displays such an under-damped $x(t)$ in green color.

In terms of colliding particles in the under-damped regime, the relative velocity of particles remains finite after the collision. Therefore, particles are still energetic, might pass a distance and endure more collisions with other particles.

\subsubsection{Critical Damping}

Over-damped and under-damped regimes are separated via the critical damping case, where $\beta^{2}-\omega_{0}^{2}=0$. For a critically damped oscillator, $\chi(t)$ can be written as

$$
x(t)=\left(A_{1}+A_{2} t\right) e^{-\beta t} .
$$

With the same initial configuration, a critically-damped oscillator reaches the equilibrium much faster than over-damped and under-damped oscillators. In Figure 4.1, temporal evolution of a critically-damped oscillator is plotted in red. 
After a short discussion about damping regimes, here we discuss about different dissipation models. To obtain the results we presented in Chapter 3, dissipation is governed by the following equation

$$
\overrightarrow{\mathrm{F}}_{\text {diss. }}=-\mathrm{b}\left[\left(\vec{v}_{i}-\vec{v}_{j}\right) \cdot \hat{r}_{i j}\right] \hat{r}_{i j}
$$

where $b$ is the damping factor and the damping force is proportional to the relative velocity of colliding particles in the direction normal to the contact point. Following the work by Vågberg [Vågberg et al., 2014] we refer to this damping model as the Contact Dissipation in normal direction $\left(\mathrm{CD}_{\mathrm{n}}\right.$ model).

Another possibility for implementing dissipation is the Contact Dissipation model, where the damping force is proportional to both normal and tangential components of the relative velocity of colliding particles. We refer to this model as CD model, the damping force is given as

$$
\overrightarrow{\mathrm{F}}_{\text {diss. }}=-\mathrm{b}\left(\vec{v}_{i}-\vec{v}_{j}\right) .
$$

The CD model was first introduced by Durian [Durian, 1997] to study the mechanics of foam bubbles under shearing. In both $\mathrm{CD}_{n}$ and $\mathrm{CD}$ models, if mass of all particles is kept fix, then the damping regime of the system is tuned by $b$.

In the $C D_{n}$ model, while the normal relative velocity of particles gets dissipated in collisions, the tangential relative velocity of particles stays untouched, even in the over-damped regime or in the case of inelastic collisions. Therefore even if the normal relative velocity gets damped completely during the collision time, the non-zero tangential velocity allows for particles to rotate around each-other and break the contact later. On the other hand, such a rotational motion is not possible in the over-damped CD model since both normal and tangential components of the relative velocity get dissipated, keeping particles in contact until an external perturbation is applied, e.g. collision with another particle.

Another possible choice of the dissipation model is the Reservoir Dissipation, which we refer to as the RD model. In this model, dissipation does not occur in contacts, as the case of $C D_{n}$ and CD models. Instead, for a shearing system with a rate of $\dot{\gamma}$, every particle with a velocity of $\vec{v}$ experiences a dissipative force as

$$
\overrightarrow{\mathrm{F}}_{\text {diss. }}=-\zeta(\vec{v}-\dot{\gamma} y \hat{x}),
$$

where $\zeta$, tuning the strength of the dissipation, is the damping factor. While $\hat{x}$ indicates the shearing direction, $y$ is the position of the particle in the shear gradient direction. In RD model, if a particle deviates from the linear flow profile, Equation 4.12 ensures that its velocity decays to the flow velocity, $\vec{v}_{\text {flow }}(y)=\dot{\gamma} y \hat{x}$. 
In dense particulate systems, RD model can be considered as a mean-field approximation of CD model [Durian, 1997; Tewari et al., 1999]. It can also model the drag force on a particles floating in a shearing fluid, such as massive particles in emulsions or suspensions [Kawasaki, Ikeda, and Berthier, 2014; Trulsson, Andreotti, and Claudin, 2012].

\subsection{DISSIPATION IN NORMAL DIRECTION}

To understand how the results presented in the previous chapter depend on energy dissipation, we vary the damping factor $b$ to explore different damping regimes. In Figure 4.2, flow-curves for a system using the $C D_{n}$ model are presented where $u=7 e-4$ and $\phi=0.75$.

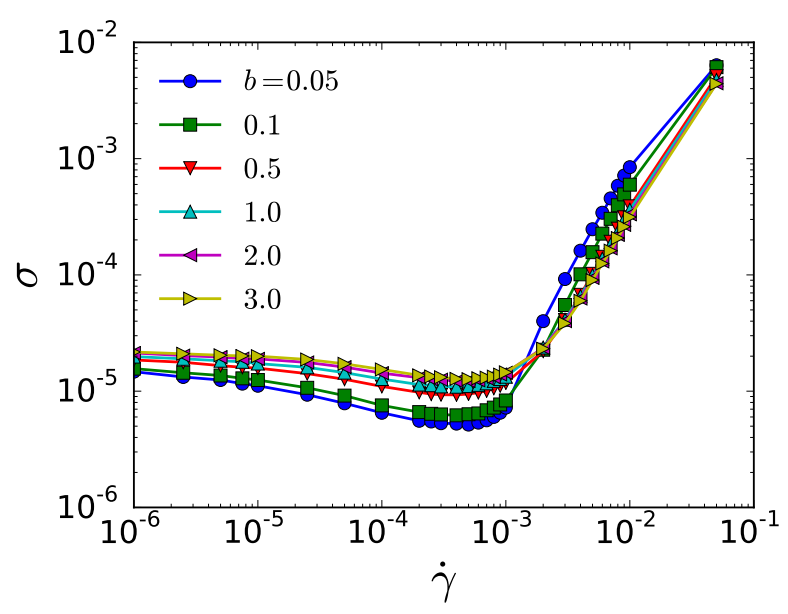

Figure 4.2: Flow-curves for a system with $\mathrm{N}=1000$, at $u=7 \times 10^{-4}$ and $\phi=0.75$.

We observe that by decreasing the damping coefficient, which eventually leads to under-damping, the non-monotonicity in the flow curves becomes more pronounced. We have already discussed the connection between the non-monotonic flow-curves, mechanical instability and shear-banding in the previous chapter. Therefore, from Figure 4.2 one can conclude that under-damping enhances mechanical instability in the system. In effect, decreasing the damping coefficient results in the increase of timescales for energy dissipation leading the system into a fluidized state for a longer period.

The dissipation time-scale is defined as

$$
\tau_{\mathrm{d}}=\frac{\mathrm{m}}{\mathrm{b}}
$$

which measures the competition between the inertia and damping. The smaller $b$ is, the larger $\tau_{d}$ gets, which means the energy is dissipated in longer times. In Figure 4.3, we rescale the imposed strain rate $\dot{\gamma}$ with the dissipation timescale $\tau_{d}$. Thereby, the data for different $b$ 
can be collapsed in the low strain-rate regime, with the tuning of $b$ leading to exploration of different regimes in this branch. Further, if $\mathrm{b}$ is small, the minimum in the flow curve occurs later, leading to the pronounced non-monotonicity.

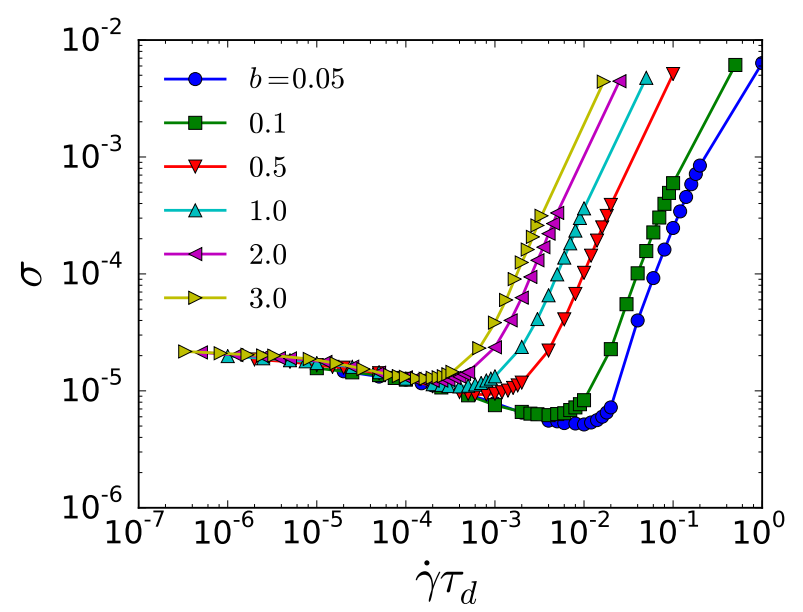

Figure 4.3: Same flow-curves as Figure 4.2, where the damping time-scale, $\tau_{d} \sim 1 / b$ is used to rescale $\dot{\gamma}$. Data points in the attractiondominated regime with different $b$, collapse on a single curve.

In simulations with the $\mathrm{CD}_{n}$ model, dissipation has more influence on the rheology of the system in the attraction-dominated regime. It can be understood by noting the fact that in the regime of small strain rates, more particles are in contact, where the damping force is also applied on particles.

We have also observed that if we look at the variation of $E_{\text {pot }}$ with changing $b$, there is a change with decreasing the imposed $\dot{\gamma}$. Typically, at large $\dot{\gamma}$, the average potential energy of the system decreases as the system gets more damped (i.e. increase of b); quicker dissipation prohibits the system from exploring all possible higher energy states. However, for $\dot{\gamma}$ in the "attractive regime", we see that $E_{p o t}$ decreases with decreasing $b$; the under-damping allowing the system to explore lower energy states in the landscape (Figure 4.4). Such a scenario has also been proposed in [Nicolas et al., 2016] to understand how damping influences steady state rheology of amorphous systems.

Figure 4.5 demonstrates the connectivity data for same systems presented in Figure 4.2. The left panel displays $z(\dot{\gamma})$ for different $b$. Smaller b leads to lower strain rate at which the isostatic structure of particles breaks down. This is expected since particles are more energetic at smaller $b$, thus are more likely to break contacts. We found the strain rate scales with $b^{-1 / 4}$. The left panel in Figure 4.5 displays how all $\delta z=z_{y}-z$ for systems with different $b$ collapse on a single curve, if the strain rate is rescaled by $b^{-1 / 4}$. However, the true 


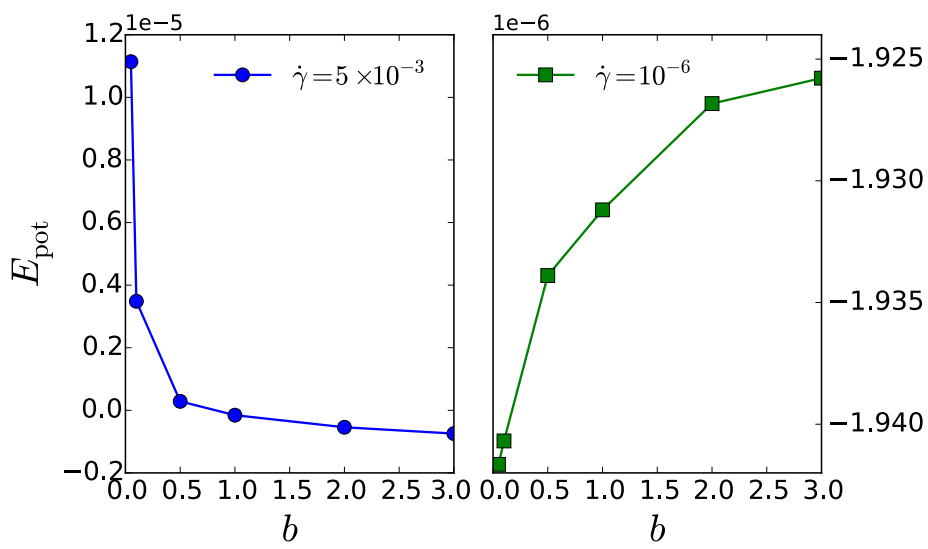

Figure 4.4: The potential energy $E_{\text {pot }}$ as a function of damping factor $b$ in the $\mathrm{CD}_{\mathrm{n}}$ model: (left) in the repulsion-dominated regime with $\dot{\gamma}=5 \times 10^{-3}$ and (right), in the attraction-dominated regime with $\dot{\gamma}=10^{-6}$.

nature of this exponent is not clear for us, needs more studies to be explained.
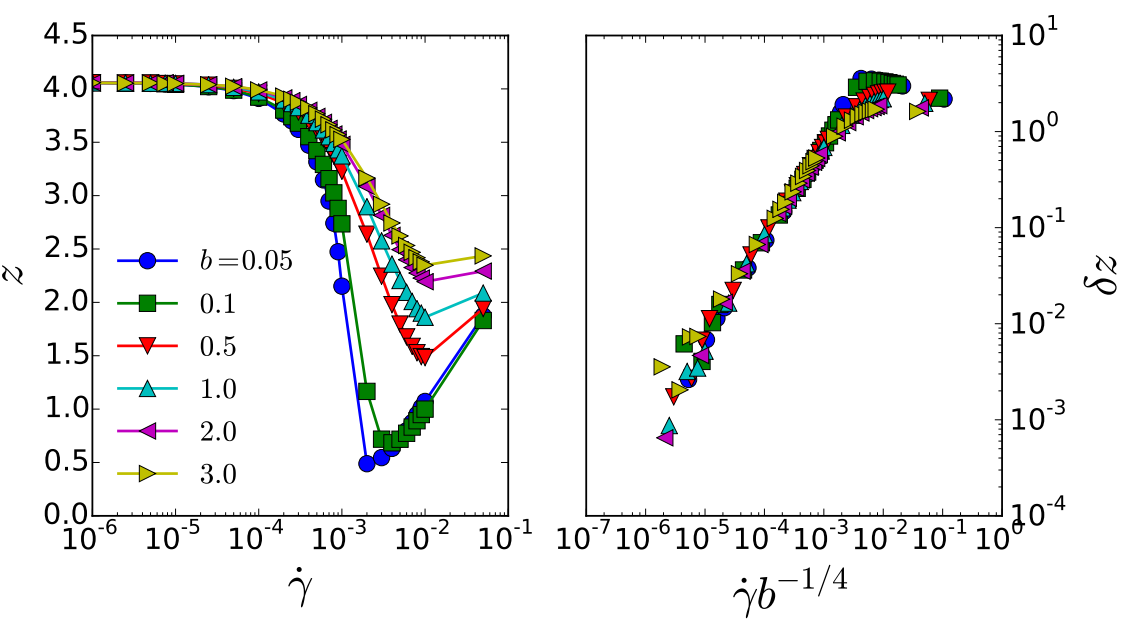

Figure 4.5: Connectivity for attractive systems $\left(u=7 \times 10^{-4}, \phi=0.75\right)$ with $\mathrm{CD}_{n}$ dissipation model, at different damping b. (Left), connectivity as a function of strain rate. (right), $\delta z$, distance to the yield connectivity, as a function of rescaled strain rate $\dot{\gamma} b^{-1 / 4}$.

\subsection{FULL CONTACT DISSIPATION}

As it is explained in Section 4.3, the Full Contact Dissipation or Contact Dissipation model ensures that the tangential relative velocity of particles gets dissipated when they collide, as well as the normal relative velocity. For the rest of this chapter, $C D$ model is used, unless mentioned otherwise. 
Rheology of repulsive systems with CD model in the over-damped regime is studied in [Vågberg et al., 2014]. There, it is shown that the tangential dissipation is crucial to give rise to viscous behaviour which leads to the linear dependence of shear stress and strain rate: $\sigma \propto \dot{\gamma}$. In the rest of this chapter, we study the effect of CD model on rheology of attractive systems in different damping regimes.

\subsubsection{Flow Curves}

Changing the dissipation mechanism from the $\mathrm{CD}_{\mathrm{n}}$ to the $\mathrm{CD}$ model alters the rheology of the system significantly. Figure 4.6 displays flow-curves of a system with $\mathrm{N}=1000$ at $\mathrm{u}=2 \times 10^{-5}$ and $\phi=0.75$ using the CD model as damping force, for different damping factors b. For the purpose of comparison, the flow-curve for the same system but with the $C D_{n}$ damping force is also presented by the dashed curved.

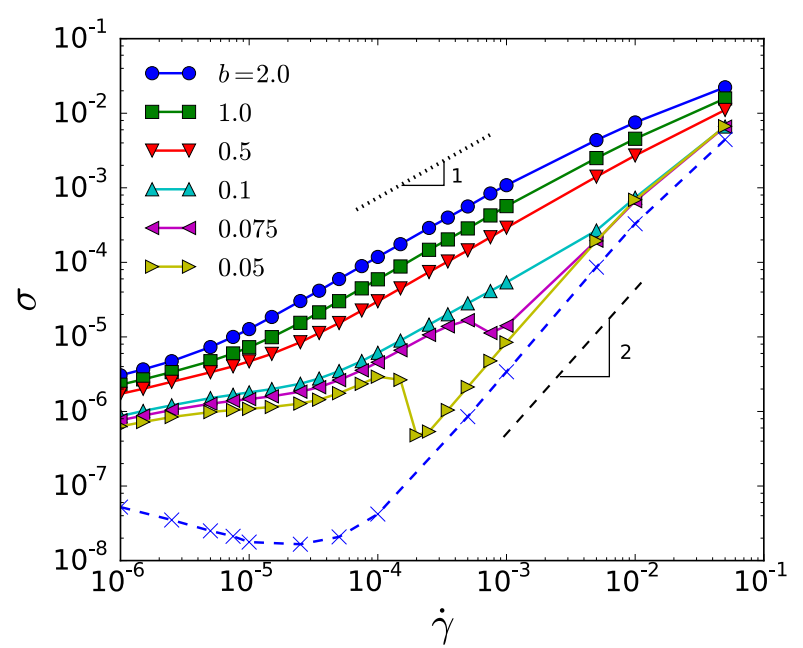

Figure 4.6: Flow-curves for a system using $C D$ model with $N=1000$, $u=2 \times 10^{-5}, \phi=0.75$ and different damping coefficient $b$. The dashed curve represents the flow-curve of the same system but using the $C D_{n}$ model with $b=2$.

In the over-damped regime $(b \geqslant 0.5)$, shear stress $\sigma$ is a monotonically increasing function of strain rate $\dot{\gamma}$. While in the limit of small strain rates the slope of $\sigma(\dot{\gamma})$ is less than 1, a viscous flow develops in the regime of intermediate strain rates which is indicated by the linear increase of shear stress with strain rate,

$$
\sigma \propto b \dot{\gamma} .
$$

This viscous flow emerges at range of strain rates where damping forces are dominant over attractive forces. Large damping, results in particles staying next to each-other after collisions, forming large clusters. Those clusters are associated with the emergence of viscous behavior [Vågberg et al., 2014]. At large strain rates, soft core effects 
become important. Particles are quite energetic to pass through eachother, leading to the weaker increase of $\sigma$ with $\dot{\gamma}$.

However, decreasing the damping factor $b$, puts the system eventually in the under-damped regime. It introduces an inertial time-scale to the system which gives rise to the inertial flow at large strain rates with the quadratic dependence of shear stress on strain rate, $\sigma \propto \dot{\gamma}^{2}$. At intermediate strain rates, flow-curves exhibit a non-monotonic transition from the viscous-like to the inertial flow.

\subsubsection{Connectivity}

Different flow properties of the system in different damping regimes, suggests that the structure of the system might also be considerably different, depending on the damping regime. To study that, we measure the connectivity of the system as a function of strain rate. Figure 4.10 demonstrates $z(\dot{\gamma})$ for the same system as Figure 4.6.

In the over-damped regime, $z$ is close but below the iso-static value $z_{\text {iso }}=4$ at small strain rates. Increasing $\dot{\gamma}$, decreases $z(\dot{\gamma})$ smoothly due to the more pronounced effect of shear-induced rupture compared to the attraction-induced aggregation. At large strain rates, $z(\dot{\gamma})$ saturates around the value 2.6 where still a percolated network of particles can be found.

In under-damped regime, $z(\dot{\gamma})$ is slightly larger than $z_{\text {iso }}$ at small strain rates which indicates the developing of an iso-static network of particles. Increasing $\dot{\gamma}$ eventually decreases $z(\dot{\gamma})$ to values below $z_{\text {iso }}$ indicating a transition from the iso-static structure to a viscous flow. Further increase of $\dot{\gamma}$ results in a sharp drop in $z(\dot{\gamma})$ : inertial flow develops. There, $z<1.0$ indicating no clusters or local structure can be found, instead of persistent contacts, binary collisions dominate the physics of the system. $z(\dot{\gamma})$ becomes an increasing function of $\dot{\gamma}$ since faster shearing brings more particles to collide.

\subsubsection{Shear Stress Ratio}

Similar to the case of Chapter 3, shear-stress ratio (Equation 3.21) measures the resistance of the system with respect to the plastic deformations under shear. Figure 4.8 represents $\mu(\dot{\gamma})$ for an attractive system $\left(u=2 \times 10^{-5}\right)$ below the jamming $(\phi=0.75)$ with different damping factors. The left and right panels correspond to over-damped and under-damped regime respectively.

Similar to attractive systems studied in Chapter $3, \mu(\dot{\gamma})$ exhibits a peak as a consequence of attractive interactions. However, the height of the peak is much smaller in the case of CD dissipation model, $\max (\mu(\dot{\gamma}))<1.1$, compared to systems with $\mathrm{CD}_{n}$ model presented in Figure 3.14. 


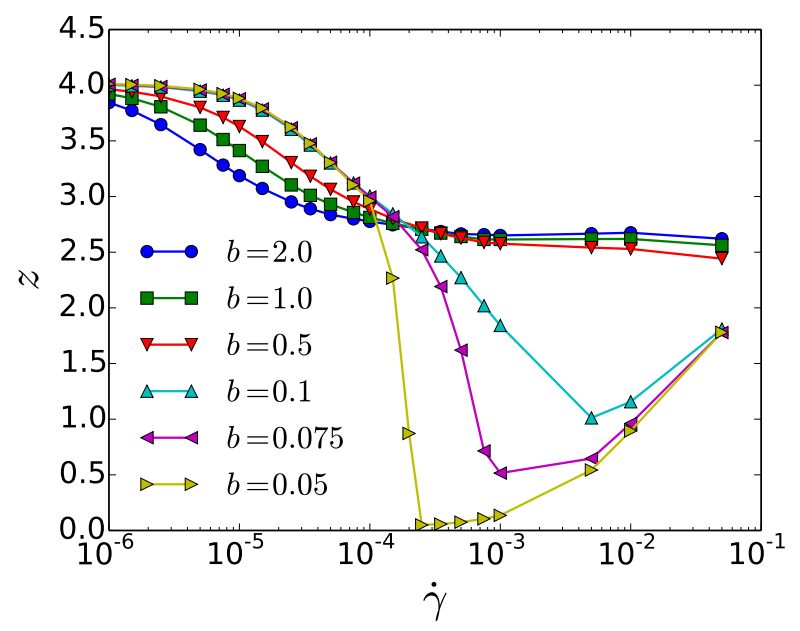

Figure 4.7: Connectivity as a function of strain rate for a system at $u=$ $2 \times 10^{-5}$ and $\phi=0.75$ with different damping factors, $\mathrm{b}(\mathrm{CD}$ model).

In the over-damped limit, $\mu(\dot{\gamma})$ exhibits a peak at small strain rates which occurs at larger $\dot{\gamma}$ as b decreases. This peak is associated with transition from the flow regime at small $\dot{\gamma}$ where $\mathrm{d} \sigma(\dot{\gamma}) / \mathrm{d} \dot{\gamma}<1$ to the viscous flow with $\mathrm{d} \sigma(\dot{\gamma}) / \mathrm{d} \dot{\gamma}=1$. In the under-damped regime, $\mu(\dot{\gamma})$ displays richer behaviour. Similar to over-damped systems, there is a peak corresponding to the transition form yield-like fluid to the viscous flow with the difference that it occurs at much larger $\dot{\gamma}$. Furthermore, the height of the peak increases with decreasing $b$. Less damped system exhibits a higher resistance to the yield-viscous flow transition. Further increase of $\dot{\gamma}$ eventually leads to the second peak in $\mu(\dot{\gamma})$ which is associated with viscous-inertial flow transition.

The observed non-monotonic form of $\mu(\dot{\gamma})$ justifies that the pressure of the system growths slower than the shear stress. To support this argument, Figure 4.9 displays $\mathrm{P}(\dot{\gamma})$ for the same systems as Figures 4.8 and 4.6. Weaker increase of $\mathrm{P}(\dot{\gamma})$ compared to $\sigma(\dot{\gamma})$ is more apparent in the under-damped regime, specifically for $b=0.05$ where in contrast to $\sigma(\dot{\gamma}), \mathrm{P}(\dot{\gamma})$ changes non-monotonically at the yield-viscous transition (around $\dot{\gamma} \approx 1.5 \times 10^{-5}$ ).

\subsubsection{Over-damped Regime: Scaling Flow Curves}

In the over-damped regime, large values of $b$ ensures that the velocity of colliding particles dissipates completely. Therefore, kinetic terms do not contribute to the shear stress and the interplay between dissipative and elastic forces characterizes the flow response.

The contribution of dissipative forces to the shear stress reads as $\sigma_{\text {diss }} \sim b \dot{\gamma}$. In our system the elastic contribution is proportional to the 

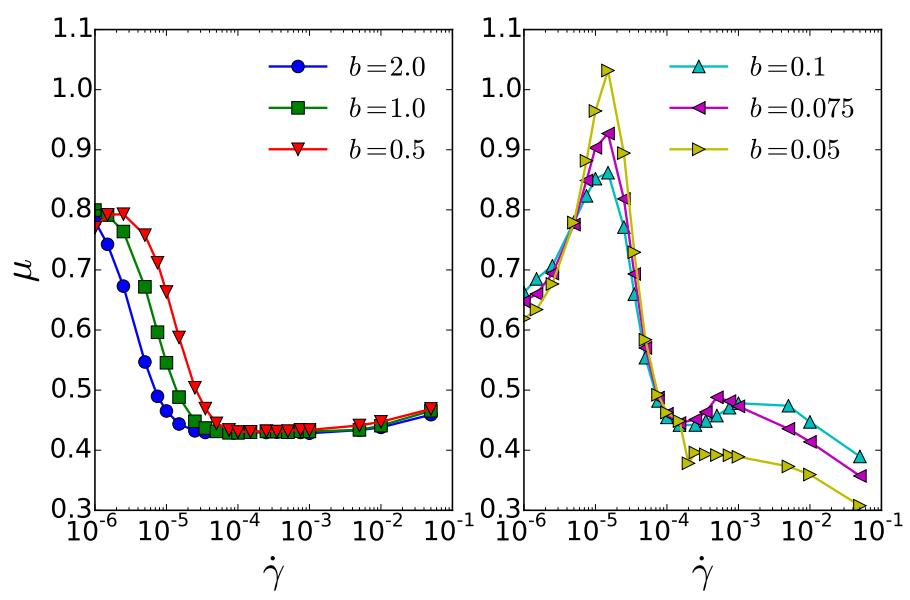

Figure 4.8: Shear-stress ratio $\mu$ as a function of strain rate for a system at $u=2 \times 10^{-5}$ and $\phi=0.75$ with different damping factors, $b$ (CD model). The left panel displays the data for over-damped systems and the right panel corresponds to the under-damped regime.

attraction strength, $\sigma_{\text {elast }} \sim \epsilon u$. Their ratio is given by the Weissenberg number

$$
W i=\frac{\sigma_{\text {diss }}}{\sigma_{\text {elast }}}=\frac{b \dot{\gamma}}{\epsilon u},
$$

which is a natural quantity to characterize the flow in the over-damped regime. This quantity can be used to collapse all flow-curves corresponding to different $\mathrm{b}$ in the over-damped regime. Figure 4.10 demonstrates such a collapse for systems with different attraction and damping, rescaling the stress by the attractive stress scale: $\sigma_{\text {att }} \sim \mathcal{u}$.

Since stress and connectivity scale with $W i$, it is reasonable to find the same scaling for the shear-stress ratio, $\mu(\dot{\gamma})$, presented is Figure 4.8. Indeed, Figure 4.II demonstrates that such a scaling collapses all $\mu(\dot{\gamma})$ on a single curve, as far as they represent systems in the overdamped regime.

\subsubsection{Under-damped Regime: Emergence of Inertial Flow}

In the under-damped regime inertial time-scale, introduced by small values of damping factor $b$, leads to the non-monotonic transition from the viscous flow $(\sigma \propto \dot{\gamma})$ to the inertial flow $\left(\sigma \propto \dot{\gamma}^{2}\right)$ with increasing $\dot{\gamma}$.

The inertial time-scale determines the strain rate at which the inertial flow governs. This time-scale is associated with the non-monotonic part in $\sigma(\dot{\gamma})$. How does this time-scale depend on system parameters like the volume fraction and the attraction strength? In other words, at a given $\phi$ and $u$, how fast shearing is required to reach the inertial flow? 


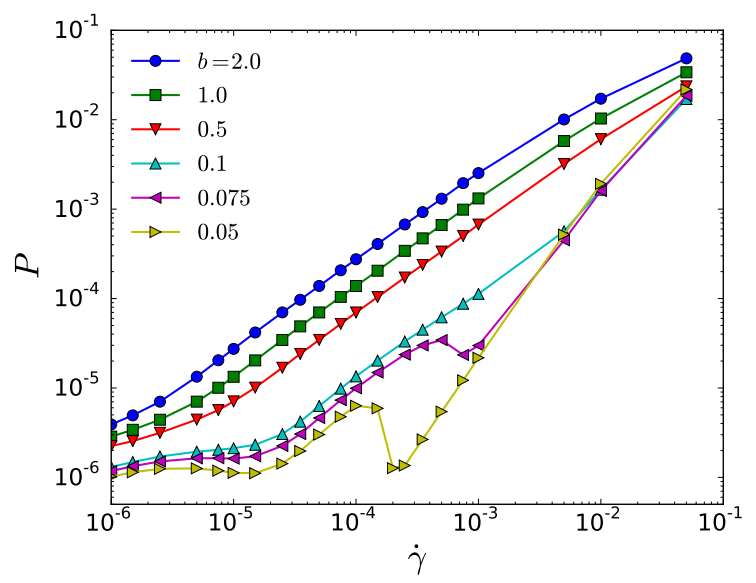

Figure 4.9: Pressure as a function of strain rate for a system at $u=2 \times$ $10^{-5}$ and $\phi=0.75$ with different damping factors, $b$ (CD model). $\mathrm{P}(\dot{\gamma})$ increases slower compared to $\sigma(\dot{\gamma})$ at the yield-viscous and viscous-inertial flow transitions.

We explore a wide range of $\phi$ for a system at a fixed $u$ and $b$ to find the effect of $\phi$ on the non-monotonic part of $\sigma(\dot{\gamma})$. Figure 4.12 illustrates flow curves for such systems with $u=2 \times 10^{-5}$ and $b=0.05$. No inertial flow and non-monotonic $\sigma(\dot{\gamma})$ is observed at volume fractions close to the jamming point $(\phi \geqslant 0.80)$. Decreasing $\phi$ eventually leads to the non-monotonic behaviour in $\sigma(\dot{\gamma})$ and an inertial flow develops at large $\dot{\gamma}$. Further decrease of $\phi$ enhances the nonmonotonicity and shifts it to smaller $\dot{\gamma}$. The more dilute the system is, the stronger the non-monotonic transition at smaller $\dot{\gamma}$ occurs. At very small volume fractions $(\phi<0.70)$, the inertial flow is dominant at the small $\dot{\gamma}$ and the viscous flow does not develop. Therefore, the non-monotonic transition occurs between the yield flow (small $\dot{\gamma}$ ) and inertial flow (intermediate and large $\dot{\gamma}$ ). On the other hand, for dense systems $(\phi>0.80)$, soft-core effects become dominant due to the large values of $\dot{\gamma}$, which prevents the inertial flow to govern. Therefore, the viscous flow governs for a long range of intermediate $\dot{\gamma}$ with shear-thinning at large $\dot{\gamma}$.

Figure 4.13 displays $\sigma(\dot{\gamma})$ for systems at $\phi=0.75$ with different $u$ and $b$. It demonstrates the role of attraction strength $u$ on the development of the inertial flow and the non-monotonic part of $\sigma(\dot{\gamma})$. For the same value of damping factor $b$, stronger attraction increases the strain rate at which the inertial flow develops. It has the same effect as increasing the volume fraction; both increase the average size of clusters made of particles which is not observed in the inertial regime. At very large $\dot{\gamma}$, flow curves collapse regardless of values of $u$ and $b$. The inertial flow and soft-core effects suppress attractive and dissipative contributions to the shear stress, only the kinetic term matters.

The effect of attraction in tuning the range of $\dot{\gamma}$ with the inertial flow can also be traced in the connectivity of the system, $z(\dot{\gamma})$. Fig- 


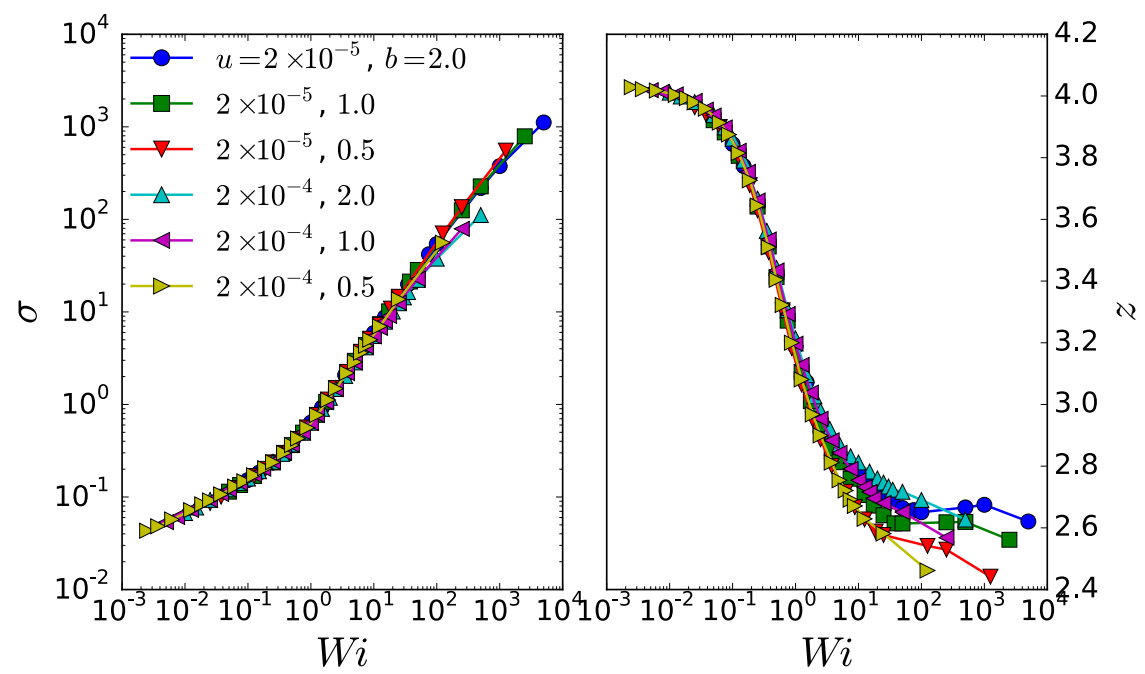

Figure 4.10: Weissenberg number, $\mathrm{Wi}=\mathrm{b} \dot{\gamma} / \epsilon u$ is used to rescale (left) flow curves and (right), connectivity of systems at $\phi=0.75$ with different attraction strength $u$ and damping factor $b$ (Overdamped regime).

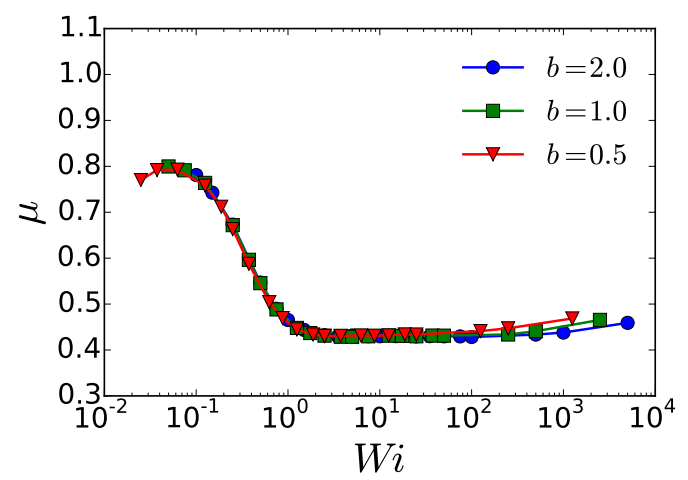

Figure 4.11: Shear-stress ratio $\mu$, for attractive systems at $u=2 \times 10^{-5}$, $\phi=0.75$, with different damping factors $b$, in the over-damped regime. Weissenberg number is used as a rescaled strain rate, resulting in the collapse of all $\mu$ on a single curve.

ure 4.14 exhibits $z(\dot{\gamma})$ for similar systems as Figure 4.13. Emergence of the inertial flow is associated with the dramatic drop of connectivity $(z<1.0)$ which indicates the formation of no persistent cluster of particles. Increasing $u$, shifts this drop to larger values of $\dot{\gamma}$.

\subsection{MECHANICAL INSTABILITY IN UNDER-DAMPED FLOW: SHEAR BANDS}

The connection between a non-monotonic $\sigma(\dot{\gamma})$ and mechanical instability has been already discussed in previous chapters, Sections 1.7 and 3.11. The previous section also characterized the emergence of 


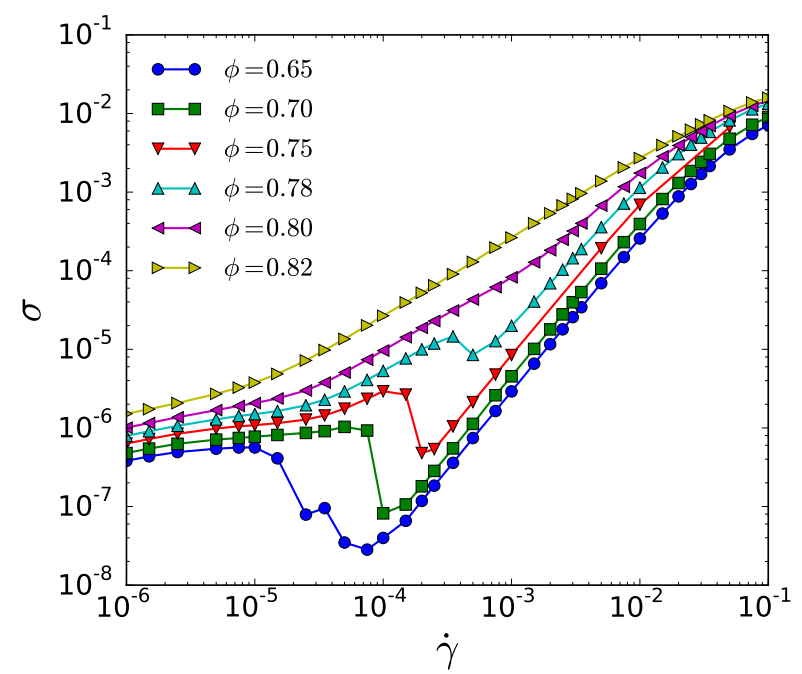

Figure 4.12: Flow curves for a system with $u=2 \times 10^{-5}$ and $b=0.05$ at different volume fractions. The non-monotonic viscous-inertial transition is shifted to smaller $\dot{\gamma}$ as the system becomes more dilute.

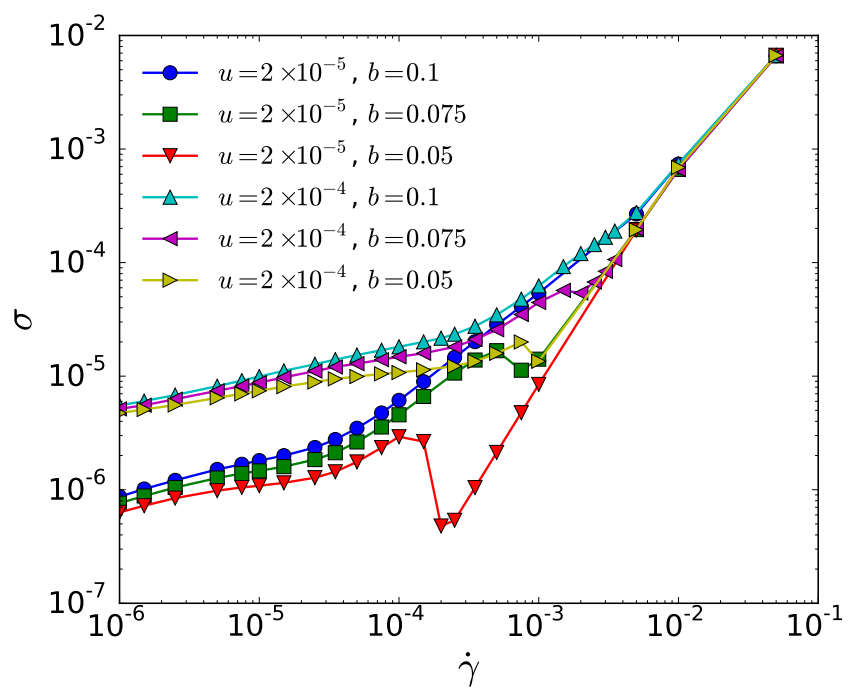

Figure 4.13: Flow curves for under-damped flows with $\phi=0.75$ but different attraction strength $u$ and damping factor $b$. Increasing the attraction strength increases the strain rate at which the nonmonotonicity in $\sigma(\dot{\gamma})$ occurs, exhibiting a viscous-inertial flow transition. 


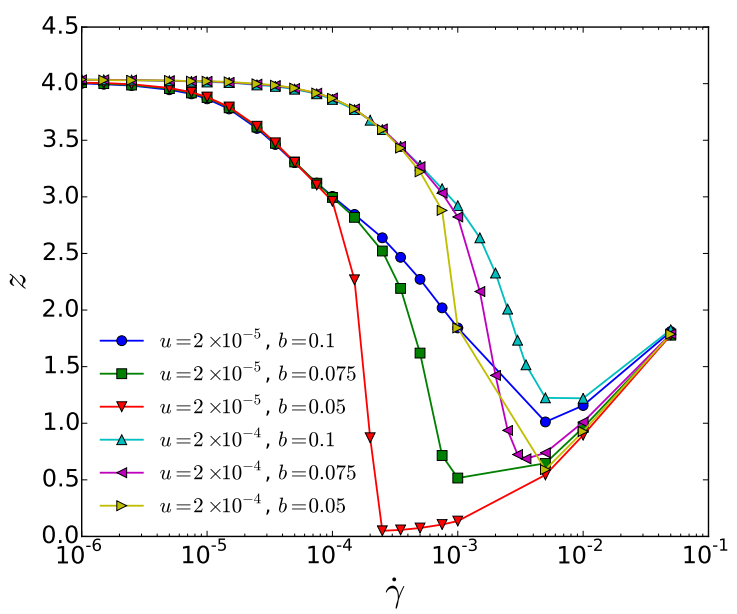

Figure 4.14: Connectivity $z(\dot{\gamma})$ for under-damped systems with $\phi=0.75$ but different attraction strength $u$ and damping factor $b$. Increasing the attraction strength increases the strain rate at which $z$ drops to the values below 1.0, corresponding to the emergence of inertial flow.

inertial flow in under-damped systems with the non-monotonic form of $\sigma(\dot{\gamma})$ before entering the quadratic regime where $\sigma \propto \dot{\gamma}^{2}$.

We observed that shear bands form at strain rates where $\sigma(\dot{\gamma})$ has a negative slope. Figure 4.15 represents the velocity, local strain rate and connectivity profiles of a shear-banded system with $\mathrm{N}=1000$ at $\phi=0.75, \mathrm{u}=2 \times 10^{-5}$ and $\mathrm{b}=0.05$, where the imposed strain rate is $\dot{\gamma}_{0}=2 \times 10^{-4}$. An inertial band with no clusters of particles $(z \approx 0.0)$ forms in coexistence with a viscous band where $z$ is finite but still below $z_{\text {iso. }}$ In the inertial band, which is highly sheared and the local strain rate reaches values of $\dot{\gamma} \sim 3 \dot{\gamma}_{0}$, the momentum exchange in binary collisions is responsible to govern the physics of the system. On the other hand, persistent contacts are dominant in the viscous band, where the local strain rate is as small as $\dot{\gamma} \sim 10^{-2} \dot{\gamma}_{0}$. Thus, contacting forces are responsible for the shear stress in the viscous band, leading to a different scale of $\sigma$ compared to the inertial band.

In comparison to shear-banding in attractive systems with the $C D_{n}$ damping (discussed in Chapter 3), a larger strain needs to be applied to the system in order to develop a steady shear localization $(\gamma \sim 15)$. This can be understood by noting that smaller $b$ leads to the increase of the time-scale for energy dissipation. Therefore the steady state is expected to reach in a longer time by imposing a larger shear strain. Hence, for every data point shown in this chapter, it was necessary to carefully check whether the stationary state is reached. 


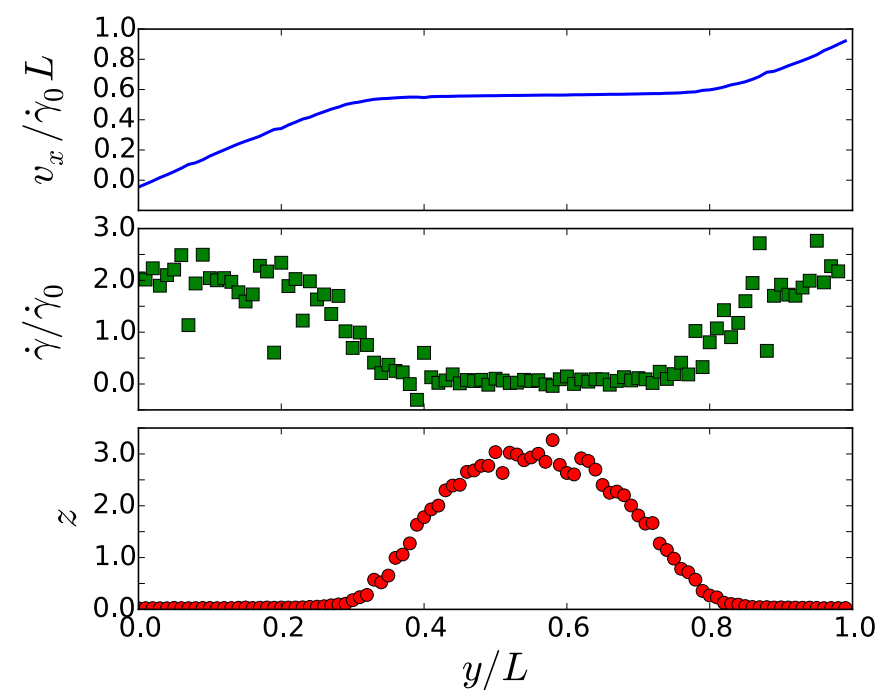

Figure 4.15: Velocity, local strain rate and connectivity profiles of a shearbanded system at $u=2 \times 10^{-5}, \phi=0.75, b=0.05$ and $\dot{\gamma}_{0}=2 \times$ $10^{-4}$. The inertial shear band $(z \approx 0.0)$ forms in coexistence with a viscous band where although a percolated structure develops, still $z<z_{\text {iso. }}$.

\subsubsection{Stress Bands: Sink of Energy}

Figure 4.16 displays a typical snapshot of the stress field and the connectivity profile for a shear-banded system at $\phi=0.75, u=2 \times 10^{-5}$ and $\mathrm{b}=0.05$, where the applied strain rate is $\dot{\gamma}=2 \times 10^{-4}$. Coloring is based on $\log (|\sigma|)$, where the white area indicates $\sigma=0$ or $\log (|\sigma|)=\infty$. There, in contrast to shear-banding in systems with $\mathrm{CD}_{n}$ dissipation model, $\sigma$ is localized. In the viscous band with a finite $z$ (large cluster of particles), $\sigma$ is determined by contacts between particles. However, those clusters do not exist in the inertial band and therefore $\sigma$ is governed by the rare binary collisions. The small and isolated colored areas in Figure 4.16 correspond to such collisions, where the spatially averaged $z$ is still close to 0.0 . The small collision frequency, which is a feature of the inertial band in our shear-banded system, results in a different level of $\sigma$, orders of magnitude smaller than $\sigma$ in the viscous band.

Averaging the stress field over a time (strain) window may govern finite local stress in the inertial band; more collisions occurs in longer time and the virial stress builds up in the inertial band. To shed a light on this point, we plot (Figure 4.17) the evolution of time-averaged stress field for the same system as Figure 4.16. We observe that even after averaging over large strains as $\Delta \gamma=2.0$, the stress field remains heterogeneous.

But how can the localized and non-uniform $\sigma$ be rationalized when we observe this shear (stress) bands are stationary and do not move in the gradient direction? Specially, how does the interface between the 


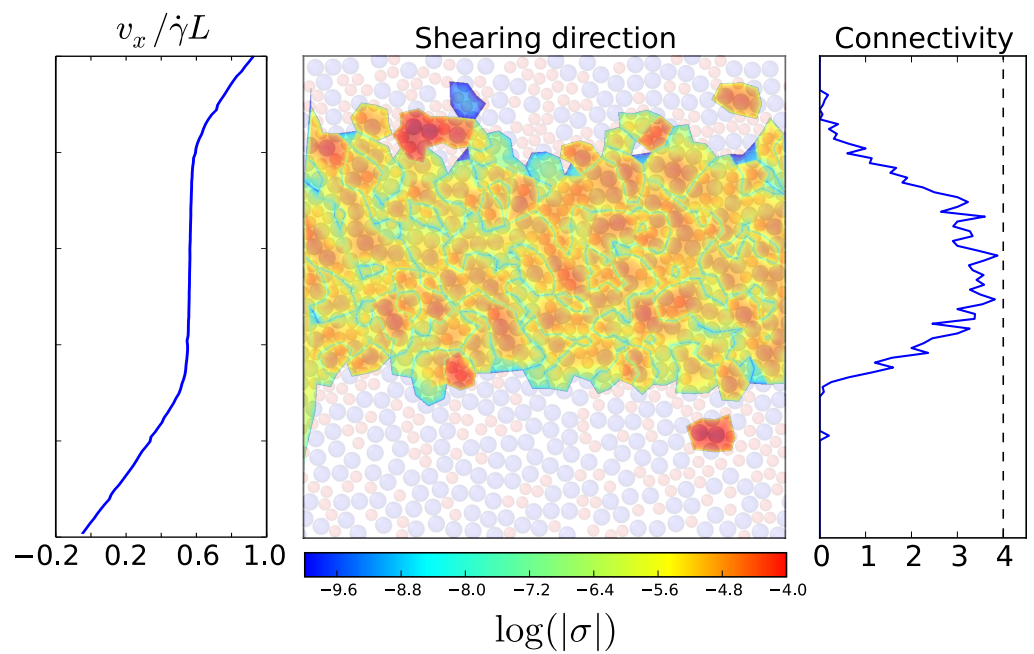

Figure 4.16: The stress field and corresponding velocity and connectivity profiles of a shear-banded system at $u=2 \times 10^{-5}, \phi=0.75$, $\mathrm{b}=0.05$ and $\dot{\gamma}=2 \times 10^{-4}$. Colored areas determine finite $\sigma$ while in the white area associated with the inertial band, $\sigma$ is orders of magnitude smaller, close to 0.0.

inertial and viscous bands stay stable, regardless of the discontinuous jump in the value of shear stress. To answer that question, we need to know that the stress field presented in Figure 4.16 is the virial term of the stress tensor, introduced in Equation 2.2. To discuss the stability of the interface between inertial and viscous band, we need also to consider the kinetic term of the stress tensor, which is the major contribution in the inertial band. Figure 4.18 displays the pressure field for the same system as Figure 4.16. Thereby, one can see while the virial pressuere (panel (a)) displays the similar discontinue jump as the virial stress over crossing the interface, the total pressure, as the kinetic term is accounted for, changes continuously over the interface, explaining its stability.

In shear-banded systems, the viscous band behaves as a sink of energy, an erodible boundary to the inertial band. In the inertial band, the relative velocity of colliding particles is likely to be finite after a collision, due to small value of $b$ and large velocity of particles. Thus, such energetic particles can endure multi collisions before heating up by shearing. However, when such an energetic particle hits the large cluster of particles in the viscous band, the particle's energy is dissipated almost compeletely over a large cluster of particles in the viscous band. This situation, having a viscous medium as an erodible boundary for an inertial rapid flow of particles, is also discussed in [Berzi and Jenkins, 2015]. 

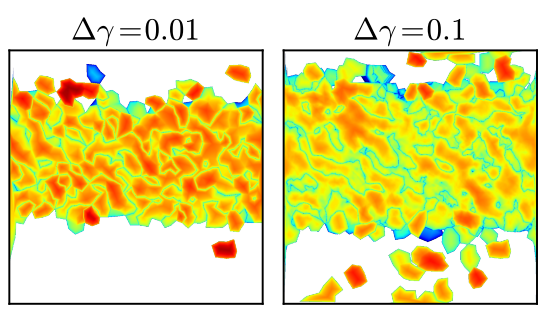

$\Delta \gamma=1.2$
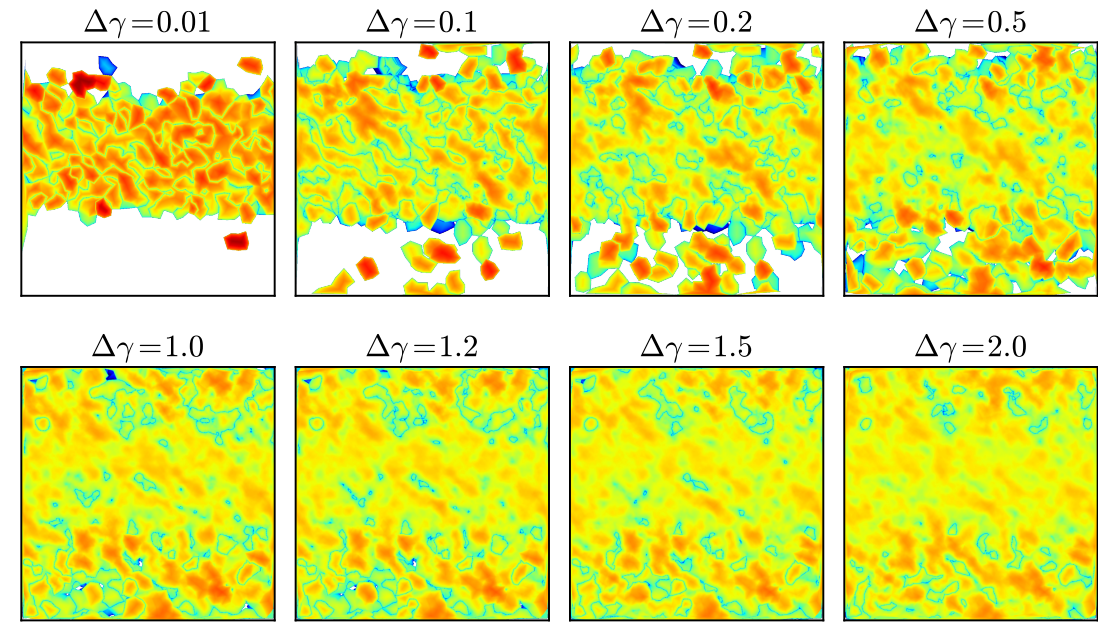

Figure 4.17: Time averaging of the stress field presented is Figure 4.16 (same color coding), over strain windows of different sizes. The stress field is heterogeneous even after averaging over $\Delta \gamma=2.0$.

(a)

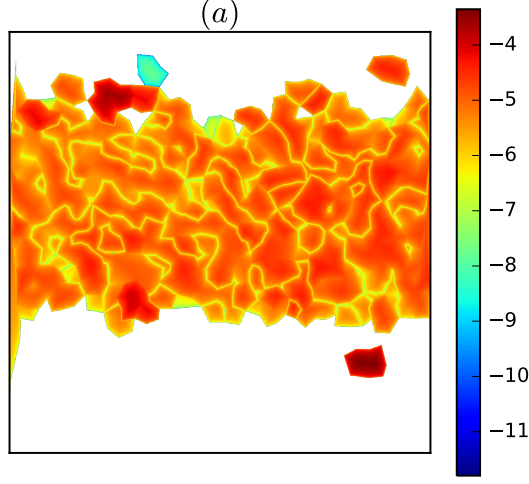

(b)

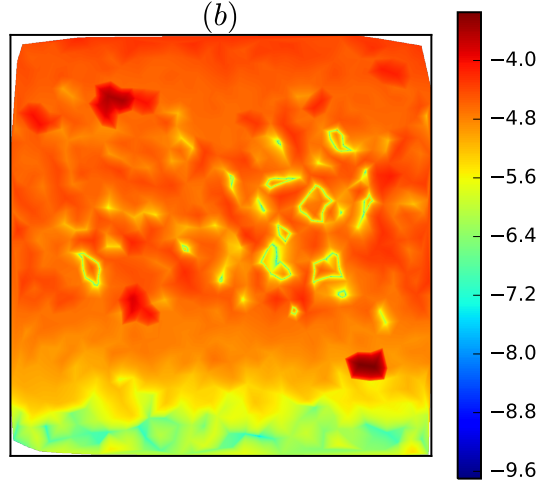

Figure 4.18: Pressure (normal stress) fields for the same system as Figure 4.16. (a) $\log (|\mathrm{P}|)$, pressure (virial) displays a similar discontinue jump at the interface. (b) $\log \left(\left|P_{\text {tot. }}\right|\right)$, total pressure (including kinetic term) continuously varies at the interface.

\subsubsection{Ramping Simulation: Hysteresis in $\sigma(\dot{\gamma})$}

Until this section, $\sigma(\dot{\gamma})$ is obtained by shearing the system at different $\dot{\gamma}$ starting from a random initial configuration with a linear velocity profile. After reaching the steady state, $\sigma$ is measured. An alternative protocol to obtain $\sigma(\dot{\gamma})$ is to start from a random configuration, measuring $\sigma$ after applying proper strains and then, increasing or decreasing $\dot{\gamma}$ gradually and repeating $\sigma$ measurement after reaching the steady state. This ramping protocol helps us to study the hysteresis in response of the system when it is shear-banded.

Figure 4.19 displays $\sigma(\dot{\gamma})$ obtained from random initial states and using the ramping method. The system is at $\phi=0.75, \mathrm{u}=2 \times 10^{-5}$ and $b=0.05$. The dashed curve indicates shearing the system at each 


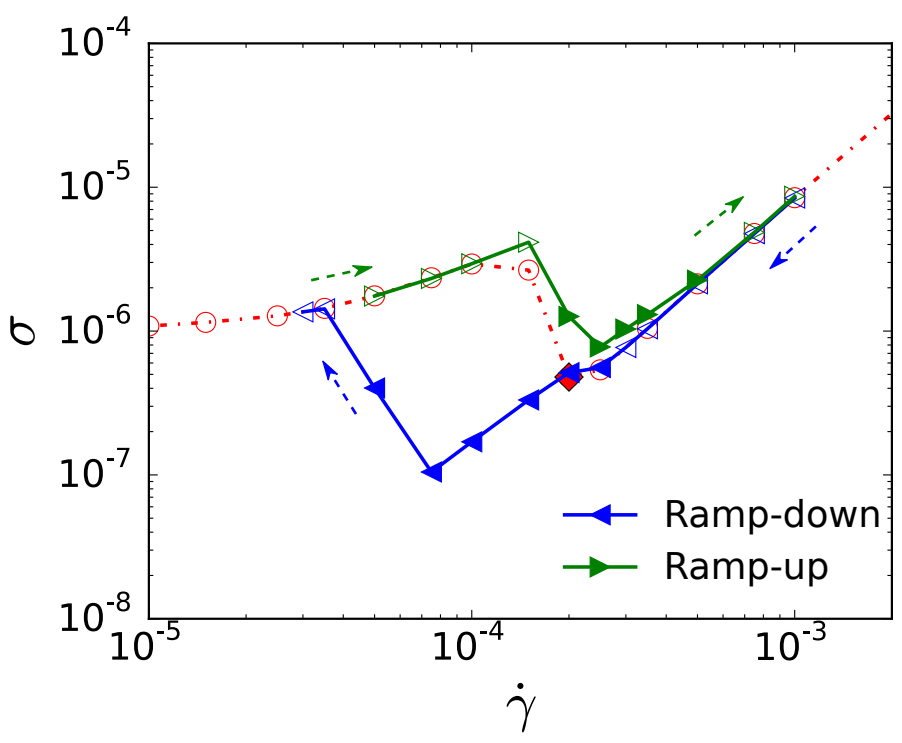

Figure 4.19: Flow curves obtained from random initial state at each $\dot{\gamma}$ and also ramping protocol for a system at $u=2 \times 10^{-5}, \phi=0.75$ and $b=0.05$. The red curve indicates the random initial onfiguration. The blue curve presents $\sigma(\dot{\gamma})$ obtained by ramping down $\dot{\gamma}$. At $\dot{\gamma}=3.5 \times 10^{-5}$ the ramping direction is reversed and the obtained $\sigma(\dot{\gamma})$ in displayed by the green curve. Filled symbols indicate strain rates at which shear-banding occurs.

$\dot{\gamma}$ starting from a random initial configuration. Solid lines represent ramping flow curves: the blue curve is obtained by ramping down $\dot{\gamma}$ to $\dot{\gamma}=3.5 \times 10^{-5}$. The ramping direction is then reversed until $\dot{\gamma}=10^{-3}$ is reached, with the flow curve presented by green. At each $\dot{\gamma}$, the system is sheared for $\gamma=40$ and $\sigma$ is measured over the last 15 strains, when the stationary state is obtained.

Different behavior of $\sigma(\dot{\gamma})$ on different ramping branches displays hysteresis in mechanical response of the system. While the rampeddown curve exhibits significant changes, the ramped-up flow curve reproduces almost the same curve as the curve obtained by using random initial states. In the ramped-down branch, the inertial band is observed to survive in the range of $\dot{\gamma}$, which is associated with homogeneous viscous flow in the ramped-up and normal flow curves $\left(\dot{\gamma}>3.5 \times 10^{-5}\right.$ and $\left.\dot{\gamma}<1.5 \times 10^{-4}\right)$. This hysterectic behavior of flow curves provides the opportunity to study and compare systems with similar set of parameters, $\phi, u, b$ and $\dot{\gamma}$ but in both homogeneous and banded states.

\subsubsection{Banded vs. Homogeneous Flow: Local Volume Fraction}

To continue the discussion given in the previous section, We investigate the behavior of the system on both ramping branches but at the same strain rate. For instance, consider the strain rate $\dot{\gamma}=5 \times 10^{-5}$, 


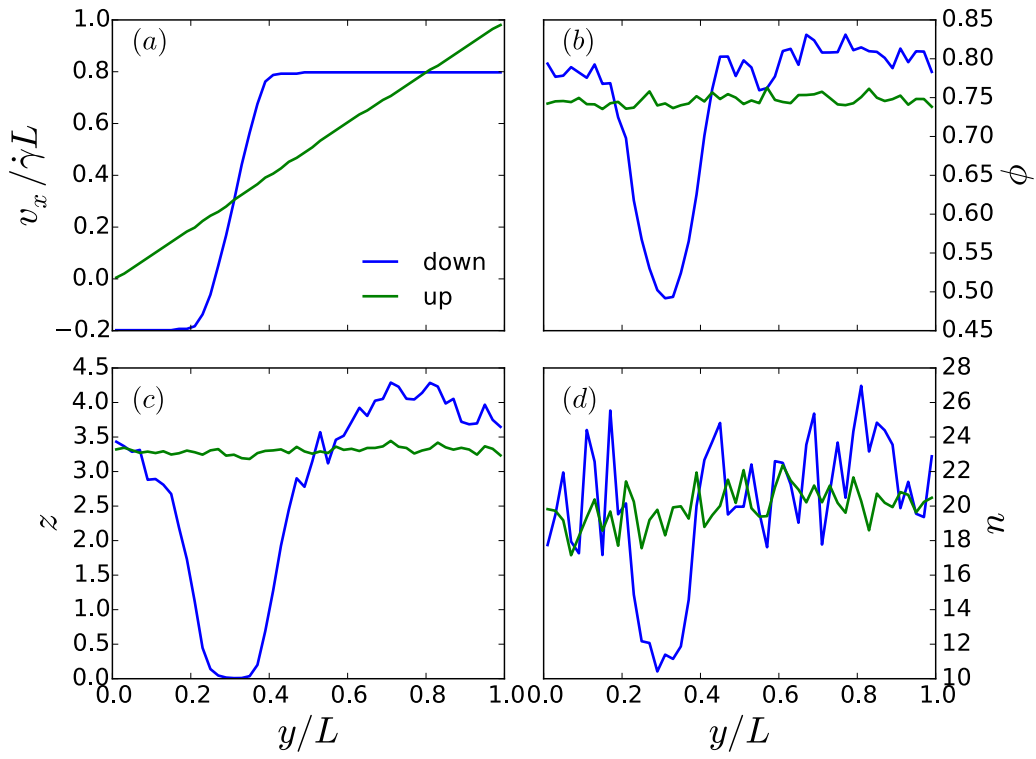

Figure 4.20: Different profiles of a system at $u=2 \times 10^{-5}, \phi=0.75$, $\mathrm{b}=0.05$ and $\dot{\gamma}=2 \times 10^{-4}$ in both shear-banded and homogeneous states. (a) displays the normalized velocity profiles, (b) presents the corresponding profile of $\phi$ which is non-uniform in the banded system. The inertial band is much more dilute than the viscous band (c) exhibits the connectivity profile and (d) demonstrates the number of particles as a function of $y$, indicating there are fewer particles in the inertial band compared to the viscous band. 
where the system is banded in the ramped-down branch but flows homogeneously on the ramped-up branch. The only relevant difference between ramping branches is the initial configuration. Therefore, one can conclude that regarding the initial configuration the system can develop shear-bands or linear velocity profile. Figure 4.20 exhibits the comparison between two different states of system, shear-banded and homogeneous flow, in terms of velocity, connectivity, local volume fraction and number of particles in the gradient direction.

Figure 4.20(a) displays velocity profiles of the system on different ramping branches at $\dot{\gamma}=5 \times 10^{-5}$. While $v_{x}(\mathrm{y})$ is linear on the rampup curve, shear bands develop in the ramp-down flow curve in form of an inertial band in coexistence with a viscous band. Figure $4.20(\mathrm{~b})$ represents the corresponding profile of local volume fraction where Voronoi tessellation is used to obtain per particle volume fraction. In the homogeneous flow, $\phi$ is uniform over the whole system and is the same as the general packing factor of the system, $\phi=0.75$. On the other hand, $\phi$ is heterogeneous in the banded system, in contrast to the case of shear-banding in systems with $\mathrm{CD}_{\mathrm{n}}$ dissipation model. The system is denser in the viscous band, $\phi(y) \approx 0.80$, but quite dilute in the inertial band where $\phi(y)<0.60$.

The emergence of non-uniform $\phi$ in shear-banded systems is associated with the development of large clusters of particles in the viscous band and the lack of those in the inertial band. Comparing Figure $4.20(\mathrm{~b})$ and (c) exhibits the link between $z(y)$ and $\phi(y)$ in shear bands. In addition, Figure 4.20 reveals that the number of particles $n(y)$ is not uniform in the shear-banded systems. The inertial band is much diluter than the viscous band not only due to the lack of particle cluster but also because of containing a smaller number of particles. While $n(y)$ is larger than 20 in the viscous band for a system with $N=1000$, in the inertial band $n(y) \approx 10-14$. Particles migrate to the viscous band, leaving the inertial band dilute.

The distribution function of $\phi$ can also display the difference between the homogeneous and shear-banded flow. Figure 4.21 illustrates $P(\phi)$, the distribution function of $\phi$ for similar systems as Figure 4.20. In the shear-banded system, $\mathrm{P}(\phi)$ is broader than the homogeneous system, and the peak of the distribution appears closer to $\phi_{\mathrm{J}}$. The dashed line indicates the system volume fraction, $\phi=0.75$.

\subsection{RESERVOIR DISSIPATION}

In the Reservoir dissipation or RD model, instead of pairs of contacting particles, dissipative force is applied to every single particle whose relative velocity with respect to the flow velocity is finite. As it is mentioned in Section 4.3, RD is proper to model particulate systems interacting with a host medium as a fluid, like emulsions and 


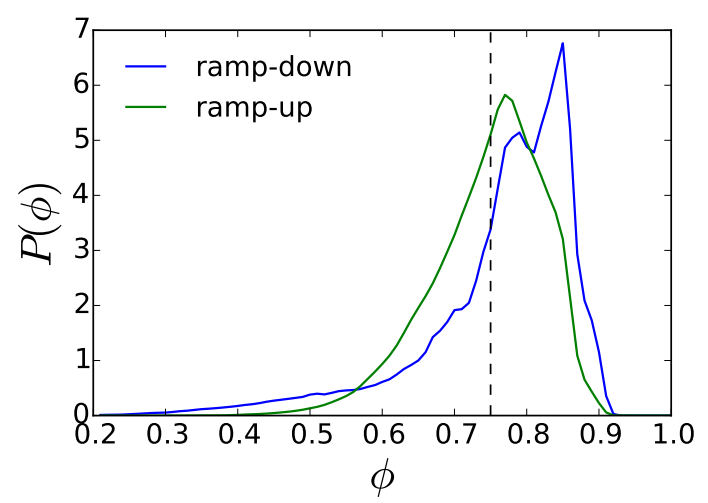

Figure 4.21: Distribution function of $\phi$ for a system at $u=2 \times 10^{-5}$, the initial volume fraction $\phi_{0}=0.75, \mathrm{~b}=0.05$ and $\dot{\gamma}=2 \times 10^{-4}$ on different ramping branches. For the shear-banded system (ramped-down branch), $\mathrm{P}(\phi)$ is wider with a peak corresponding to the denser $\phi$ with respect to $\mathrm{P}(\phi)$ in the homogeneous system (ramped-up branch).

suspensions. In this section, we report our numerical results, using RD model.

\subsubsection{Flow Curves}

Here, we consider two situations for the dissipation model. One is using the combination of $\mathrm{RD}$ and $\mathrm{CD}_{n}$ models, where $\mathrm{RD}$, chracterized by $\zeta$, corresponds to the drag force imposed by the flow and $C D_{n}$, is responsible for dissipation in particles collisions. In $\mathrm{CD}_{n}$, Equation 3.2, the damping factor $b$, tunes how elastic collisions are.

For repulsive systems, we found that $b$ affects the rheology only at relatively large strain rates. Figure 4.22 displays flow curves for repulsive systems $(u=0.0)$ at $\phi=0.75$, for different $\zeta$ and $b$.

Left panel of Figure 4.22 reveals that while $\zeta$ is fixed, $C D_{n}$ damping force governs Bagnold scaling $\left(\sigma \propto \dot{\gamma}^{2}\right)$ at large strain rates. In the right panel, where $b=2.0, \mathrm{RD}$ dissipative force results in the emergence of viscous behavior at small strain rates, which is resembles by the linear dependence of shear stress to strain rate, $\sigma \propto \zeta \dot{\gamma}$. Increasing $\zeta$, extends the range of strains at which the viscous behavior is observed. In that regime, we found that stress scales with the damping factor $\zeta$, which is expected due to the domination of dissipative forces at small strain rates. Increasing $\dot{\gamma}$ eventually leads to a crossover from viscous to inertial flow, which is observed in different experiments and numerical studies[Fall et al., 2010; Trulsson et al., 2012].

A valid question is how attractive interactions interply with $R D$ dissipative forces? In order to answer this question, we turn on attraction in systems which are presented via Figure 4.22, with over- 


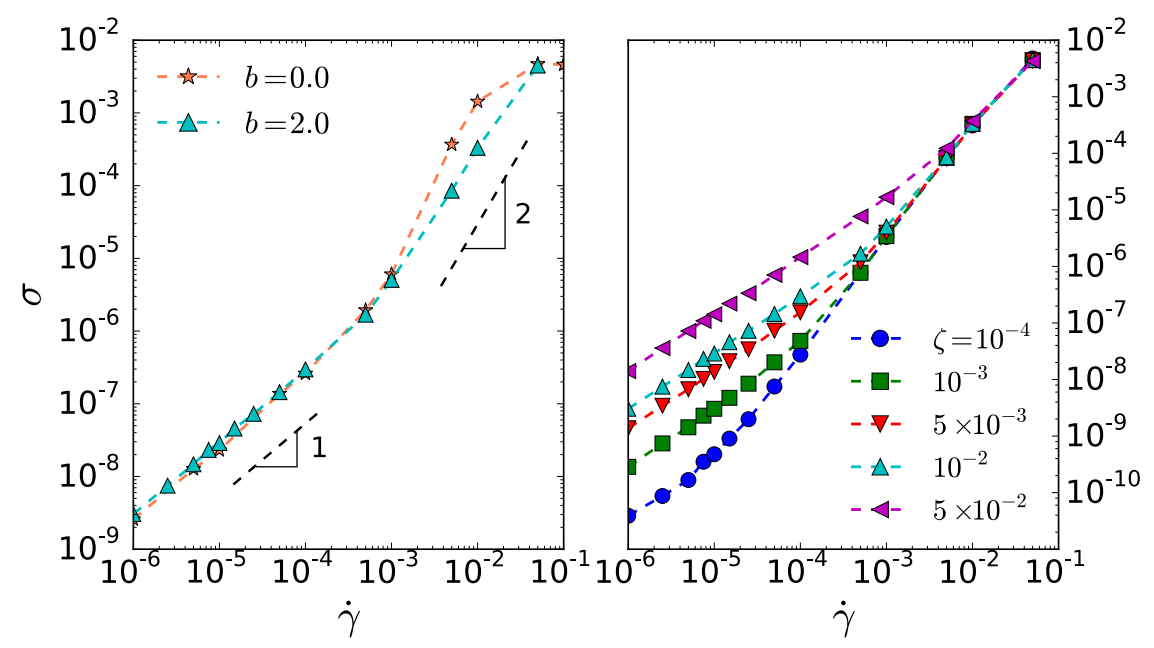

Figure 4.22: Flow Curve of repulsive systems $(u=0.0)$ at $\phi=0.75$ with RD and $\mathrm{CD}_{\mathrm{n}}$ dissipation models. (Left), Flow curves obtain with $\zeta=10^{-2}$ and diffrent $\mathrm{b}$. (Right), Flow curves obtained with $\mathrm{b}=2.0$ and different $\zeta$.

damped collisions $(b=2.0)$. Resulting flow curves are displayed in Figure 4.23. The right panel exhibits flow curves of attractive systems at $u=2 \times 10^{-5}$ and $\phi=0.75$, while repulsive flow curves are plotted via dashed lines for comparison. The left panel compares flow curves obtained with different attraction range, solid and dashed lines represent respectively $u=2 \times 10^{-5}$ and $u=2 \times 10^{-4}$. In both panels, different colors indicate different values of damping factor, $\zeta$.

Figure 4.23 demonstrates that increasing the damping factor $\zeta$, weakens the non-monotonic part of the flow curve. For $\zeta \geqslant 5 \times 10^{-2}$, the non-monotonic part of the flow curve completely disappears. Therefore, shear-banding is expected to be observed in under-damped system with $\zeta<5 \times 10^{-2}$. In addition, Figure 4.23 illustrates that all flow curves of systems with the same $u$ but different $\zeta$ head to a common limiting stress, the yield stress, at the limit of zero strain rate. This observation can be understood by noting the fact that the yield stress, $\sigma_{y}=\lim _{\dot{\gamma} \rightarrow 0} \sigma(\dot{\gamma})$, does not depend on details of the dissipative force but only properties of the material, such as the range of attraction.

\subsubsection{Viscosity: Thinning and Thickening}

Flow curves presented in the previous subsection, can be also expressed in terms of viscosity,

$$
\eta(\dot{\gamma})=\frac{\sigma(\dot{\gamma})}{\dot{\gamma}}
$$

(1.6 revisited.)

Using Stokes law, the solvent viscosity can be defined as $\eta_{\mathrm{s}}=$ $\zeta / 3 \pi\left\langle r_{c}\right\rangle$, which we use as the natural unit of viscosity. For system with RD dissipation model, two relevant time-scales can be defined. 


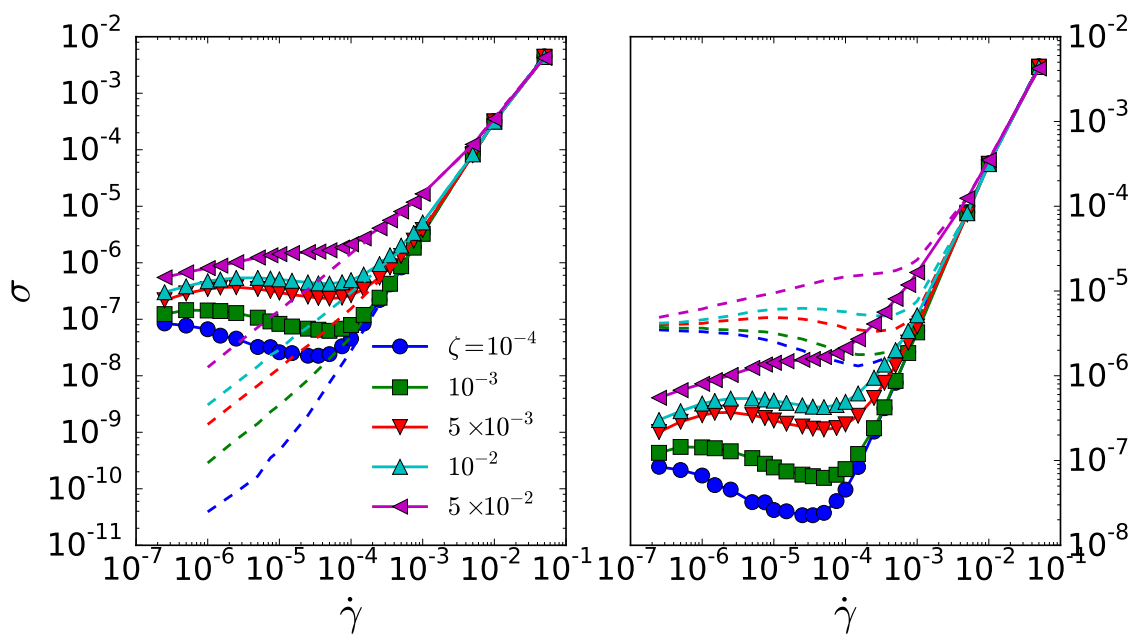

Figure 4.23: Flow curve for attractive systems at $\phi=0.75$ with both RD and $C_{n}(b=2)$ dissipation models. (Left), attraction range is set to $u=2 \times 10^{-5}$, while repulsive flow curves are plotted as dashed curves for comparison. (Right), Attractive flow curves for $u=2 \times 10^{-5}$ (solid lines) and $u=2 \times 10^{-4}$ (dashed lines). In both panels, different colors indicates different $\zeta$.

The time-scale corresponding to the damping of particle's velocity is given by,

$$
\tau_{\mathrm{d}}=\frac{\mathrm{m}}{\zeta}
$$

where $m$ is the mass of particles. For repulsive systems presented in Figure 4.22 (right), strain rate can be rescaled with $\tau_{d}$ to collapse all viscosity data points on a single curve.

For attractive systems, such scaling presented in Figure 4.24 works only at large strain rates, where the attraction is unimportant. Due to the complicated form of $\sigma(\dot{\gamma})$ at small strain rates, where attraction is dominated, we can not find a proper scaling relation to obtain the same data collapse as Figure 4.24 for small strain rates. However, it is expected to observe shear-thinning at small strain rate: at the limit of zero strain rate, an isostatic structure develops due to attractive interactions. Increasing the strain rate, as we discussed in Chapter 3, weakens the stability of this structure, reducing the viscosity of the system. The shear-thinning regime extends to the strain rates at which attractive interactions become unimportant; the repulsion-dominated regime. There the system effectively flows as a repulsive fluid, increasing the strain rate leads to more collisions among particles and consequently, more resistance against flow.

Furthermore, we found that increasing $\zeta$ in attractive systems weakens both shear-thinning and thickening regime. However, with increasing $\zeta$, the maximum of $\eta(\dot{\gamma})$ in shear-thickening regime decreases faster. As a result, for $\zeta \geqslant \zeta^{*}$, which we have found to correspond to the over-damped regime, the shear-thickening regime disappears 


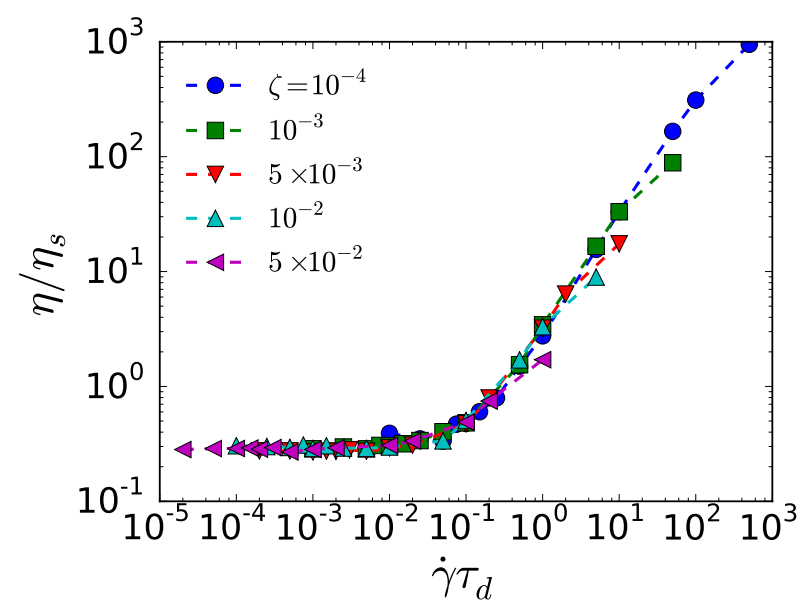

Figure 4.24: viscosity of repulsive systems with different damping factor $\zeta$, as a function of rescaled strain rate, $\dot{\gamma} \tau_{\mathrm{d}}$.

completely. Figure 4.25 displays such a variation in rheology of attractive systems with altering $\zeta$. The right panel, exhibits $\sigma(\dot{\gamma})$ for systems at $u=2 \times 10^{-5}, \phi=0.75$ and $b=0.0$ (no dissipation in collisions) with altering $\zeta$. The left panel, illustrates corresponding viscosities. For these set of parameters, we found $\zeta^{*}=1.0$, above which the thickening regime vanishes. Similar findings are also reported in a numerical study of repulsive suspensions, in which the shear-thickening regime vanishes as the jamming volume fraction is approached from below [Kawasaki et al., 2014]. 


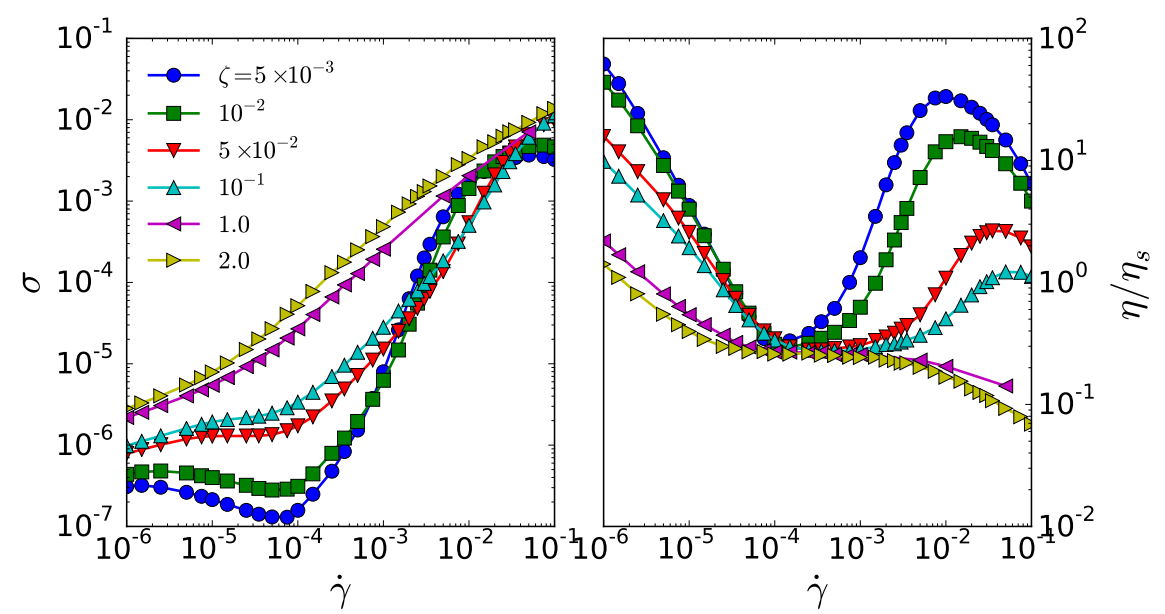

Figure 4.25: Flow curves of attractive systems with RD model, at $u=2 \times$ $10^{-5}$ and $\phi=0.75$, for different damping factors, $\zeta$. (Right), shear stress as a function of strain rate, (Left), corresponding viscosities. 


\subsection{INTRODUCTION}

In the previous chapters, we established a framework to study the rheology of weakly attractive particles in the athermal regime. It has been shown how varying small details of particles interaction like the attraction range or the form (and strength) of the damping force leads to the rich rheological response of the system. In the limit of zero temperature, typical size of particles is assumed to be large enough to have them insensitive to thermal forces, for instance Brownian forces.

However, large groups of disordered soft material (colloids, polymers, etc.) are subject to thermal forces. In the context of thermalsensitive materials, the emergence of solidity is usually associated with the glass transition. Many studies are performed to understand the differences in rheology of repulsive soft materials, in the context of athermal and thermal regimes, where the dynamics of the system is affected by jamming transition and the glass transition respectively [Ikeda et al., 2012; 2013]. In this chapter, we focus in the role of thermal forces on the rheology of attractive systems.

Thereby, we introduce a minimal model to mimic thermal fluctuations in contact forces. Then, we explore a wide range of temperature values, along the crossover from the athermal limit to the completely thermal-dominated regime. Flow curves, the system structure and particle dynamics are discussed in different regimes. The chapter ends with a section regarding the phase separation.

\subsection{RANDOM FORCES: IMPLEMENTATION}

Particles $i$ and $j$ interact via:

$$
\vec{F}=\vec{F}_{i j}^{C}+\vec{F}_{i j}^{D}+\vec{F}_{i j}^{R}
$$

where $\mathrm{F}_{i j}^{\mathrm{C}}$ is the conservative force derived from the potential introduced in Equation 3.1. The $C D_{n}$ dissipation model is used to govern the viscous damping force,

$$
\overrightarrow{\mathrm{r}}_{i j}^{\mathrm{D}}=-\mathrm{b}\left[\left(\vec{v}_{i}-\vec{v}_{j}\right) \cdot \hat{r}_{i j}\right] \hat{r}_{i j}, \quad \text { (3.2 revisited.) }
$$

when particles overlap: $r_{i j} \leqslant R_{i}+R_{j}\left(R_{i}\right.$ is the radius of $i$ th particle). To introduce a temperature to the system, the random force $\vec{F}_{i j}^{R}$ is 
exerted on overlapping particles as a thermal noise. To satisfy the fluctuation-dissipation theorem, the thermal noise is governed as

$$
\overrightarrow{\mathrm{F}}_{i j}^{R}=\alpha \sqrt{\frac{2 \mathrm{bK} \mathrm{B}}{\Delta t}}
$$

with $\alpha$ being a Gaussian random number with zero mean and unit variance. $T$ indicates the temperature, $K_{B}$ represents the Boltzman constant and $\Delta t$ is the simulation time step.

If $u$ determines the range of attraction, $\epsilon u^{2}$ is the attractive energy scale. We introduce parameter $\tau$ as

$$
\tau=\frac{K_{B} T}{\epsilon u^{2}}
$$

in order to measure the thermal energy in the unit of attractive energy scale.

\subsubsection{Thermal Stress and Thermal Time Scales}

By introducing the thermal energy $\left(\mathrm{K}_{\mathrm{B}} \mathrm{T}\right)$ to the system, we can define characteristic time and stress scales. Here, as we mentioned above, dissipation $\left(\mathrm{CD}_{n}\right.$ model $)$ and random forces are applied only to particles which are in contacts. In $\mathrm{CD}_{n}$ model, since the tangential velocity does not get damped, inertia is important and the damping time-scale is given by Equation 4.13. Therefore, the microscopic time scale for the thermal-induced motion of particles is

$$
\mathrm{t}_{\mathrm{T}}=\mathrm{r}_{\mathrm{c}} \sqrt{\frac{\mathrm{m}}{\mathrm{K}_{\mathrm{B}} \mathrm{T}^{\prime}}}
$$

which corresponds to the time that a particle moves a distance equal to its diameter due to the thermal energy. In over-damped systems with $\mathrm{RD}$ or $\mathrm{CD}$ as an alternative dissipation model, $\mathrm{t}_{\mathrm{T}}$ is given by

$$
\mathrm{t}_{\mathrm{T}}=\frac{\zeta \mathrm{r}_{\mathrm{c}}^{2}}{\mathrm{~K}_{\mathrm{B}} \mathrm{T}^{\prime}}
$$

where $\zeta$ is the damping factor in Equations 4.12 and 4.11. In this Chapter, $t_{T}$ refers always to Equation 5.4 unless it is explicitly mentioned otherwise.

Likewise, the contribution of thermal fluctuations to the stress can be quantified by means of a stress scale,

$$
\sigma_{\mathrm{T}} \sim \frac{\mathrm{K}_{\mathrm{B}} \mathrm{T}}{\mathrm{r}_{\mathrm{c}}^{3}} .
$$

The stress scale due to attractive forces is $\sigma_{a}=\epsilon u^{2} / r_{c}^{3}$. Thus, the thermal stress can be written as $\sigma_{\mathrm{T}}=\tau \sigma_{\mathrm{a}}$. 


\subsection{FLOW CURVES: FROM ATHERMAL TO THERMAL SYSTEMS}

We want to understand how the rheology of attractive systems changes in crossover from the athermal to the thermal limit. In order to do so, we vary $\tau$ from $1.25 \times 10^{-2}$ to $10^{2}$ and obtain $\sigma(\dot{\gamma})$ at each $\tau$ separately. Figure 5.I displays $\sigma(\dot{\gamma})$ for such systems with $u=2 \times 10^{-4}$ at $\phi=0.75$ (panel (a)) and $\phi=0.82$ (panel (b)). The stress is measured in units of thermal stress-scale, $\sigma_{\mathrm{T}}$ and the strain rate is rescaled by the thermal time-scale, which is now called the Pećlet number $\mathrm{P}_{\mathrm{e}}=\mathrm{t}_{\mathrm{T}} \dot{\gamma}$. In thermal fluids, Pećlet number $P_{e}$, measures the ratio of the shearinduced motion characterized by $\dot{\gamma}$, to the diffusion resembled by $t_{\mathrm{T}}$.

Figure 5.1 demonstrates three distinct regimes of flow curves for both $\phi=0.75$ and $\phi=0.82$. At very small temperatures, $\tau<1.25 \times$ $10^{-1}, \sigma(\dot{\gamma})$ almost reproduces the athermal flow curve due to negligible contribution of random forces to the development of the shear stress. The isostatic network of particles at small $\dot{\gamma}$ and the non-monotonic behavior of $\sigma(\dot{\gamma})$ are observed in that regime similar to results discussed in Chapter 3. Thus, we refer to this regime as the athermal regime.
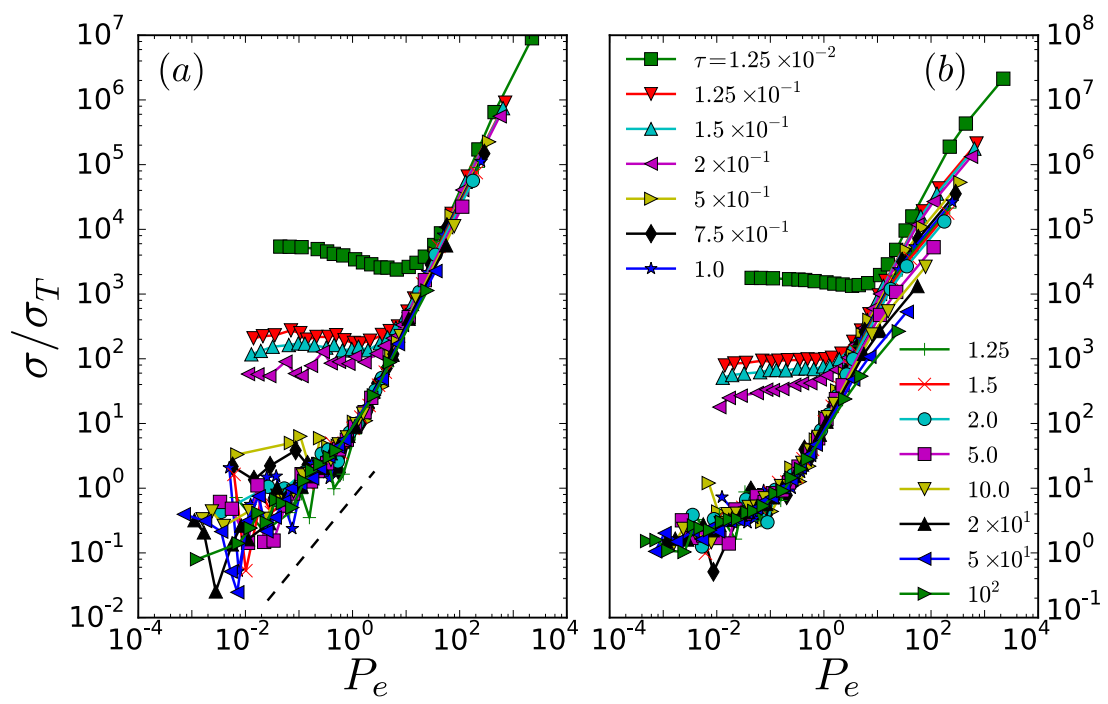

Figure 5.1: Flow curves for a system with $N=1000, u=2 \times 10^{-4}$ at (a) $\phi=0.75$, (b) $\phi=0.82$ and different temperatures $\tau$. Stress is presented in units of thermal stress scale $\left(\sigma_{T}=\epsilon u^{2} \tau / r^{3}{ }_{c}\right)$ and the strain rate is rescaled by the thermal time-scale $\left(\mathrm{t}_{\mathrm{T}}=\right.$ $\left.r_{c}\left(m / K_{B} T\right)^{1 / 2}\right)$.

Another rheological regime is reached by slightly increasing $\tau$. At $1.25 \times 10^{-1} \leqslant \tau \leqslant 2 \times 10^{-1}$, the yield stress and the non-monotonic part of $\sigma(\dot{\gamma})$ is less pronounced giving the hint that the isostatic structure of particles gets weaker due to increasing effect of thermal fluctuations, yielding less stress compared to the athermal system. This regime is called the intermediate regime. 
Further increase of $\tau$ leads the system towards the glassy regime. For $\tau \geqslant 0.5$, the shear stress is mainly determined by the thermal term therefore all $\sigma\left(\dot{\gamma} t_{T}\right)$ at different $\tau$ collapse on a single curve which is determined by $\phi$ and $u$. In Figure 5.I(a), where $\phi=0.75$, the system flows as a viscous fluid, $\sigma / \sigma_{\mathrm{T}} \sim \mathrm{P}_{e}$, for low strain rates. On the hand for $\phi=0.82$, the rescaled stress approaches a finite value, the yield stress, in the limit of zero strain rate. These different behaviors correspond to the emergence of solidity above glass transition. For our systems, the glass transition is estimated to occurs at $\phi_{\mathrm{G}} \approx 0.80$. Therefore, in the glassy regime $\left(\tau \geqslant 2 \times 10^{-1}\right)$, systems with $\phi>\phi_{\mathrm{G}}$ behaves as yields stress flow, while system with $\phi<\phi_{\mathrm{G}}$ flow as Newtonian fluids.

\subsubsection{Stress in Attractive Regime vs. $\tau$}

It is already shown in Figure 5.I that increasing $\tau$, reduces the rigidity and the amount of the stress that the network of particles can yield before entering the glassy regime. This can be understood by noting the fact that thermal fluctuations tend to destroy contacts between particles. But how this tendency is mirrored in the functional form of the yield stress with respect to increasing $\tau$ ?

We define $\sigma_{0}$ as the value of shear stress in the limit of zero strain rate,

$$
\sigma_{0}=\lim _{\dot{\gamma} \rightarrow 0} \sigma(\dot{\gamma})
$$

In our simulations, we approximate $\sigma_{0}$ by shear stress at the smallest strain rate at which we shear the system, $\sigma_{0} \approx \sigma\left(\dot{\gamma}=10^{-6}\right)$. In the attractive regime, where $\tau \ll 1.0$, and systems above the $\phi_{\mathrm{G}}$, Equation 5.7 measures the yield stress, $\sigma_{y}=\sigma_{0}$.

Figure 5.2 represents how $\sigma_{0}$ changes with $\tau$, for an attractive system with $\mathrm{N}=1000$ and $u=2 \times 10^{-4}$, at $\phi=0.75$ and $\phi=0.82$. For small values of $\tau(\tau \ll 1)$, attractive forces play a major role to determine the physics of the system. However, we found that below the jamming point, increasing $\tau$ leads to the exponential decay of $\sigma_{0}(\tau)$ due to increasing likelihood of thermally-excited particles to escape attractive bonds.

Further increase of $\tau$ runs the system toward a regime where thermally induced collisions become dominant. Therefore the linear form of $\sigma_{y}(\tau)$ is expected which is presented by the dashed line in Figure 5.2.

In addition to thermal effects, we observed that $\sigma_{0}$ scales linearly with $\delta \phi^{-1}$, with $\delta \phi$ measuring the distance to the jamming point. In Chapter 3, we already discussed the scaling behavior of athermal $\sigma_{0}\left(\sigma_{y}\right)$. Below the jamming point and for athermal systems, Equation 3.16 expresses the attractive yield stress scales as $\sigma_{\mathrm{y}} \sim u^{1 / 2} \delta z^{3 / 2}$. 


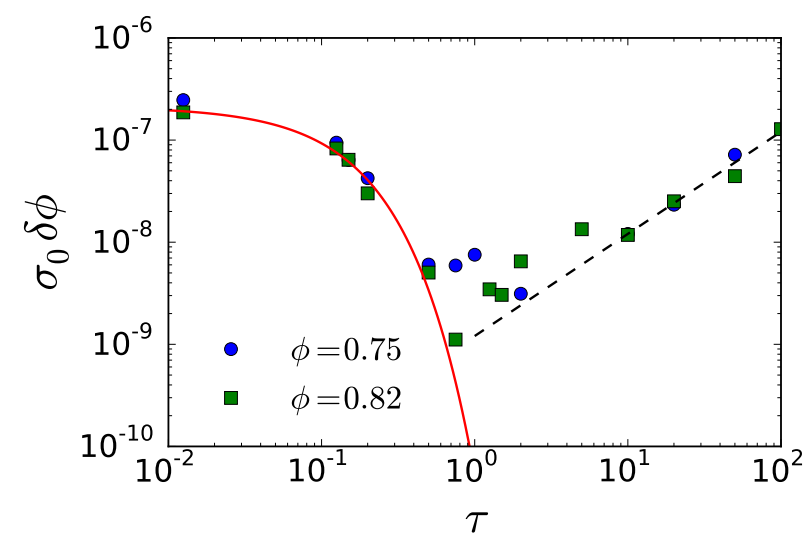

Figure 5.2: The yield stress $\left(\sigma_{y}\right)$ times the distance to the jamming point $(\delta \phi)$, as a function of $\tau$ for systems at $\phi=0.75$ and $\phi=0.82$. The range of attraction is set to $u=2 \times 10^{-4}$. For $\tau<1$ (attractive regime), $\sigma_{y}$ exhibits an exponential decay with increasing $\tau$ (solid red line). For $\tau>1$ (thermal regime), the shear stress displays a linear increase with $\tau$ (dashed line).

Hence, we can conclude that in the athermal limit, $\delta z^{3 / 2} \sim \delta \phi^{-1}$. Indeed, we found this scaling relation to hold for small values of $\delta z$, corresponding to very low strain rates. Therefore, the stress in the limit of zero strain rate, $\sigma_{0}$, can be written in terms of $\tau$ and $\phi$ as

$$
\sigma_{y}(\phi, \tau) \propto \begin{cases}\delta \phi^{-1} \cdot e^{-a \tau} & \tau \ll 1 \\ \delta \phi^{-1} \cdot \tau & \tau \geqslant 1 .\end{cases}
$$

\subsubsection{Potential Energy vs $\tau$}

In a similar way, $E_{0}$, is defined as the potential energy per particle at the limit of zero strain rate. Since we always shear the system with a finite rate, $E_{0}$ can be estimated by the value of $E$ at the smallest $\dot{\gamma}$ we use

$$
\mathrm{E}_{0}=\lim _{\dot{\gamma} \rightarrow 0} \mathrm{E}(\dot{\gamma}) .
$$

In the attractive regime, or above the jamming point, $E_{0}$ measures the yield potential energy per particle, $\mathrm{E}_{\mathrm{y}}=\mathrm{E}_{\mathrm{O}}$.

Figure 5.3 represnts $E_{0}(\tau)$ for a system with $u=2 \times 10^{-4}$ at two volume fractions, $\phi=0.75$ and $\phi=0.82$. For $\tau \leqslant 1.25 x^{-1}, E_{y}$ is negative which is expected due to the dominated role of attraction, and does not change with increasing $\tau$, indicating the athermal regime. For $1.25 \times 10^{-1}<\tau \leqslant 1.0$, there is an intermediate regime, where the system is affected by both attraction and thermal fluctuations. Thus $E_{0}(\tau)$ is an increasing function which becomes eventually positive. For larger values of temperature, $\tau>1.0, E_{y}$ is a positive and monotonically increasing function of $\tau$, where thermal fluctuations 


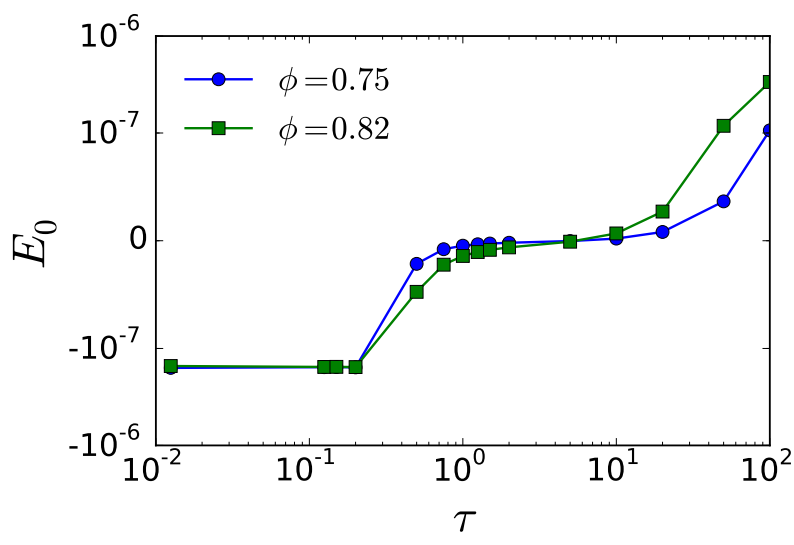

Figure 5.3: The potential energy at the limit of zero strain rate, $\left(E_{0}\right)$, as a function of $\tau$ for systems at $\phi=0.75$ and $\phi=0.82$. The range of attraction is set to $u=2 \times 10^{-4}$.

dominate the physics of the system, indicating the thermal regime. In this regime, we found the potential energy per particle grows with $\tau$ as

$$
\mathrm{E}_{0}(\tau) \propto \tau^{1.7}
$$

\subsection{CONNECTIVITY}

The connectivity, $z(\dot{\gamma})$, gives information about the micro-structure of the system. For example, if the system behaves as a rigid material, the connectivity of the system needs to exceed the isostatic value: $z>z_{\text {iso }}$. This is the case for attractive systems we studied in previous chapters in the athermal regime.

Figure 5.4 displays how the connectivity $z(\dot{\gamma})$ is altered if thermal forces are introduced to the system. The attraction range is $u=$ $2 \times 10^{-4}$ and different colors correspond to different temperature $\tau$. The system in panel (a) has $\phi=0.75$ which is below $\phi_{\mathrm{G}}$ whereas in panel (b) the system is above the glass point, $\phi=0.82>\phi_{\mathrm{G}}$. Figures 5.5 and 5.6 represent the same data in three-dimensional plot, where the color coding indicates the value of $z$. At small $\tau$, attractive forces are the main player resulting in the nearly-isostatic structure with $z>z_{\text {iso }}$ at small $\dot{\gamma}$. Increasing $\dot{\gamma}$ leads to the attractive-repulsive transition corresponding to the shear-induced destruction of the isostatic network of particles. At a given $\dot{\gamma}$, increasing $\tau$ leads to the athermal-thermal transition at which similarly, the isostatic structure is destroyed but due to thermal fluctuations.

At small $\dot{\gamma}$, the system is characterized by an isostatic network of particles at small $\tau$. The athermal-thermal transition coincides with the sharp drop in $z$ since thermal fluctuations tend to destroy the attractive bonds. Figures 5.4, 5.5 and 5.6 show that at the limit of zero strain rate, the drop in $z$ is sharper for a system at $\phi=0.75$, where $z \approx z_{\text {iso }}$ 

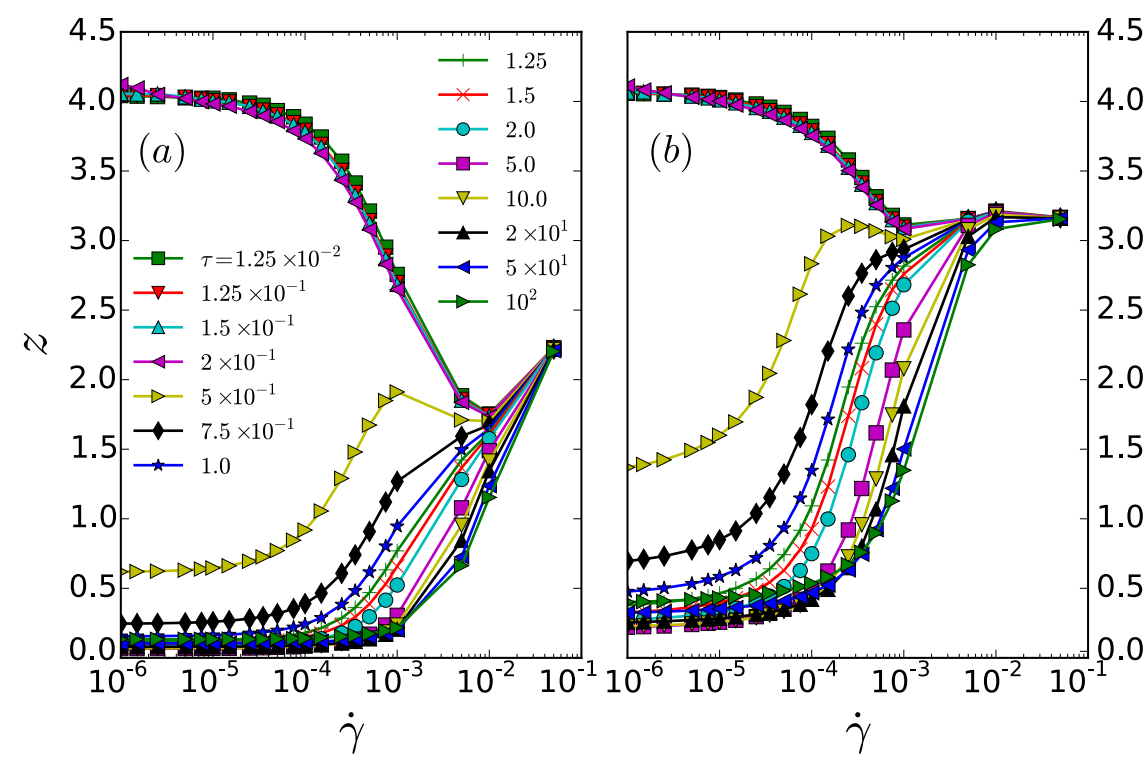

Figure 5.4: Connectivity $z$ as a function of $\dot{\gamma}$ for an attractive system at different $\tau$ and (a) $\phi=0.75$ (b) $\phi=0.82$. The attraction range is set to $u=2 \times 10^{-4}$.

jumps to $z<1.0$. In addition, at large $\dot{\gamma}$, the variation of $z$ in the athermal-thermal transition also depends on $\phi$. If the system is below the glass point, thermal fluctuations reduce $z$ by destroying repulsive contacts of particles induced by fast shearing. Such a behavior can be easily seen in Figure 5.5 , for example by looking at $z\left(\dot{\gamma}=10^{-2}\right)$ on the $\tau$ axis. On the other hand if $\phi>\phi_{\mathrm{G}}$, thermal fluctuation are responsible for the emergence of rigidity and repulsive contacts in the thermal regime. Figure 5.6 displays such a situation, where for instance $z(\dot{\gamma}=10 \times-2)$ is not changed with varying $\tau$, even after the athermal-thermal transition.

Following the definition given in Chapter 3, Figure 5.7 demonstrates the variation of the yield connectivity as $\tau$ is changed. Different types of connectivity are also plotted via dashed lines. Figure 5.7 reveals that in the regime of small $\tau$, repulsive and attractive connectivity act differently with varying $\tau$. Increasing $\tau$, reduces the number of repulsive contacts and increases the number of attractive contacts. It can be understood by noting the fact that a pair of particles is subjected to random forces only if they have a repulsive contact. Thus, thermal forces tend to increase the distance between particles, changing the type of the contact from repulsive to attractive.

An interesting observation is that at $\tau=2 \times 10^{-1}$, just at the edge of thermal regime, the number of repulsive and attractive contacts roughly equals. The qualitative explanation is given in the following. For an isostatic-like structure of particles like what we have in the attractive regime (small $\tau$ and $\dot{\gamma}$ ), repulsive contacts are essential for the stability. To have a pair of particles with a repulsive contact getting 


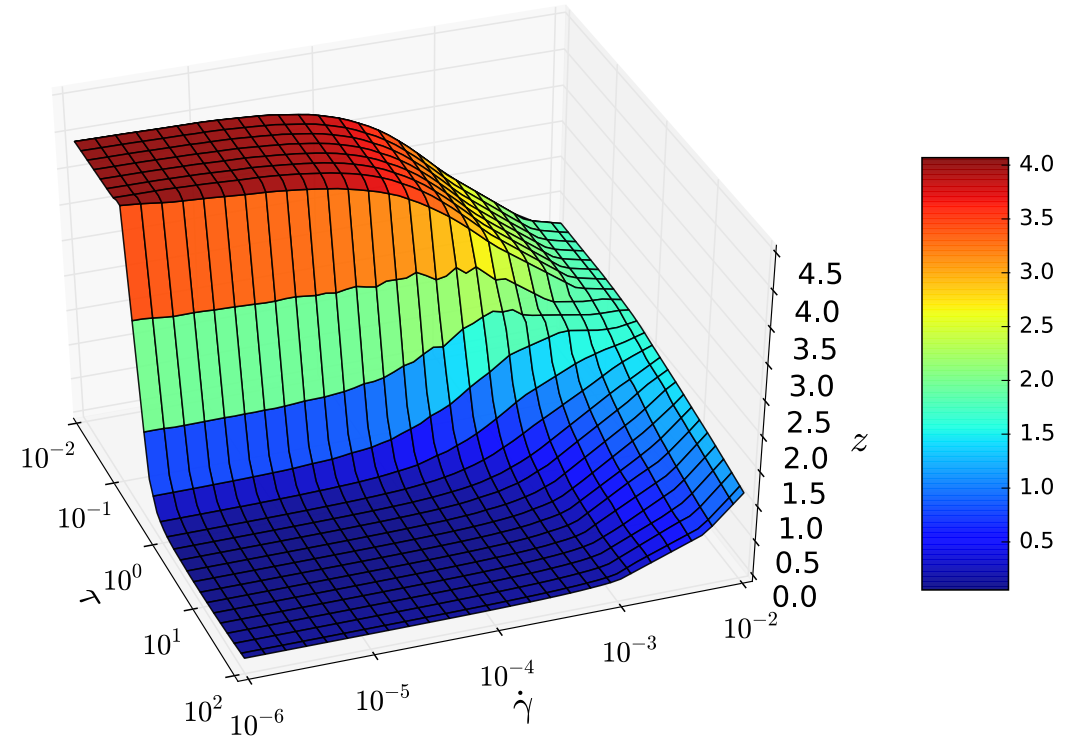

Figure 5.5: $z(\tau, \dot{\gamma})$ for an attractive system at $u=2 \times 10^{-4}$ and $\phi=0.75$. For such a system below the glass point $\phi_{\mathrm{G}}, z(\tau)$ decreases at the athermal-thermal transition.

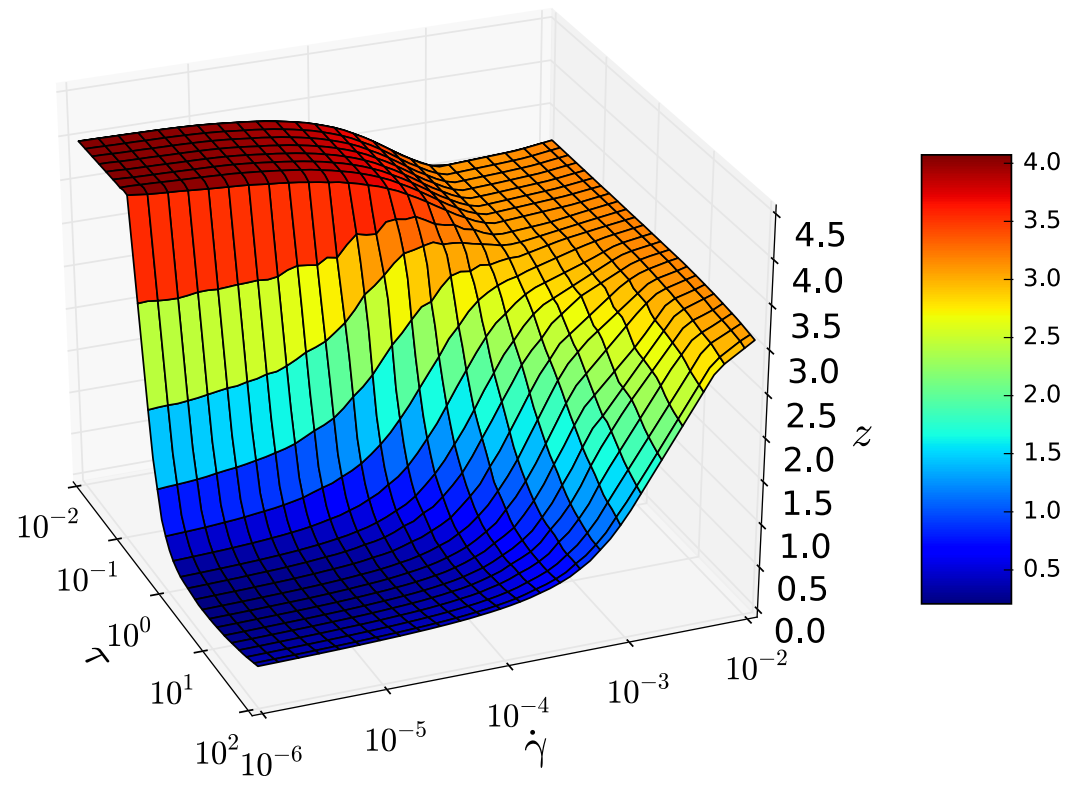

Figure 5.6: $z(\tau, \dot{\gamma})$ for an attractive system at $u=2 \times 10^{-4}$ and $\phi=0.82$. Relatively large values of $z$ is observed at fast shearing, regardless of $\tau$. At small $\tau$ shear-induced collisions are responsible for keeping connectivity large, $z \approx 3$ and at large $\tau$, thermal collisions. 


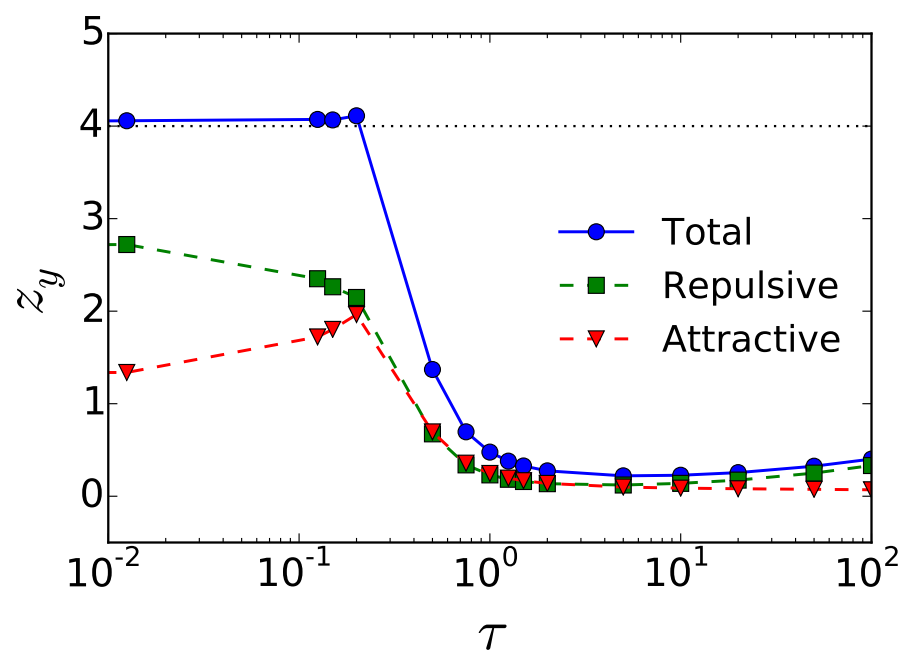

Figure 5.7: The yield connectivity $z_{y}$ as function of effective temperature, $\tau$, for an attractive system at $u=2 \times 10^{-4}$ and $\phi=0.82$. The total connectivity is plotted by blue solid line, the repulsive connectivity is indicated by the green dashed line and the attractive connectivity is represented by the red dashed line.

apart, the contact needs to first convert to an attractive contact. When particles push each-other away, a damping force dissipates their normal relative velocity. Thus, particles are less energetic just after the repulsive contact becomes attractive. In addition, the harmonic attractive force pushes particles back to each-other. Therefore, repulsive contacts are more enduring and stable than attractive ones. Note that in the case of attractive contacts, no damping force is applied to particles. Thus, they can escape the bond easier, due to shearing or collisions with other particles. More ever, we observe that in the attractive regime (small $\tau$ and $\dot{\gamma}$ ), the system self organizes to an isostatic structure with $z \approx z_{\text {iso }}$. Since repulsive contacts first become attractive and then get broken, it is reasonable and also observed in our simulations, that the variation in the number of repulsive and attractive contacts with changing $\tau$ are the same but in opposite directions,

$$
\Delta z_{\text {rep }} \approx \Delta z_{\text {att }}
$$

while the system is still in the attractive regime. We argue that repulsive contacts are essential for stability of the isostatic structure and increasing $\tau$ tends to decrease the number of them. Therefore, at the edge of the thermal regime, where even slight increase of $\tau$ leads to complete destruction of the isostatic structure, one can conclude that $z_{\text {rep }} \approx z / 2$, which immediately gives

$$
z_{\text {rep }} \approx z_{\text {att }} .
$$

Further increase of $\tau$ leads to the discontinues athermal-thermal transition with a huge drop of connectivity, indicating the destruction of 
the micro-structure of the system due to dominated thermal fluctuations $\left(z \ll z_{\text {iso }}\right)$.

\section{$5 \cdot 5$ STRUCTURE OF THE SYSTEM}

We already discussed how the structure and arrangements of particles are affected by attraction, in different flow regimes. In this section we investigate the effect of thermal fluctuations on those properties of the system.

\subsubsection{Structure Factor}

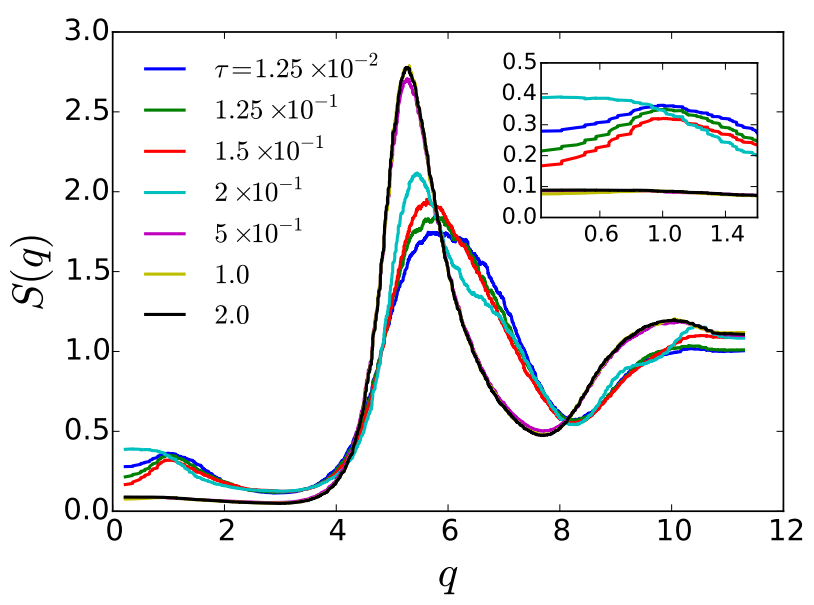

Figure 5.8: The structure factor $S(q)$ for a system at $u=2 \times 10^{-4}, \phi=$ 0.75 and different $\tau$. The small peak, corresponding to clusters of particles, disappears as $\tau$ moves towards the thermal regime.

The structure factor of the system, $S(q)=N^{-1}\langle\rho(q) \rho(-q)\rangle$, provides us with information about the local arrangement of particles. Figure 5.8 reveals $\mathbf{S}(\mathbf{q})$ for systems at different temperature but similar strain rate, $\dot{\gamma}=10^{-6}$, where attractive forces compete with thermal fluctuations. At small $\tau, S(q)$ exhibits similar behavior to athermal system. Attractive forces lead to the formation of clusters whose size is associated with the first small peak in $S(q)$ for $q<2.0$. However, increasing $\tau$ eventually decreases $q$, indicating that at $\dot{\gamma}=10^{-6}$, the size of clusters grow with $\tau$. Further increase of $\tau$ puts the system in the thermal regime where random forces overcome attractive forces $\left(\tau>2 \times 10^{-1}\right)$. In this regime, thermal fluctuations destroy the static structure of the system and $S(q)$ for different $\tau$ collapse on the top of each other with no peak at the limit of small q. In this regime,since the attraction is not relevant any more, the system behaves as a repulsive glassy system under shear with a well studied structure factor. 
In line with the case of athermal systems discussed in Chapter 3, here by obtaining partial structure factors, we have found that small particles have a significant contribution to the first peak of the structure factor. Figure 5.9 exhibits partial structure factors of system presented in Figure 5.8, with $S_{00}(q)$ and $S_{11}(q)$ are obtained respectively only for small and large particles.
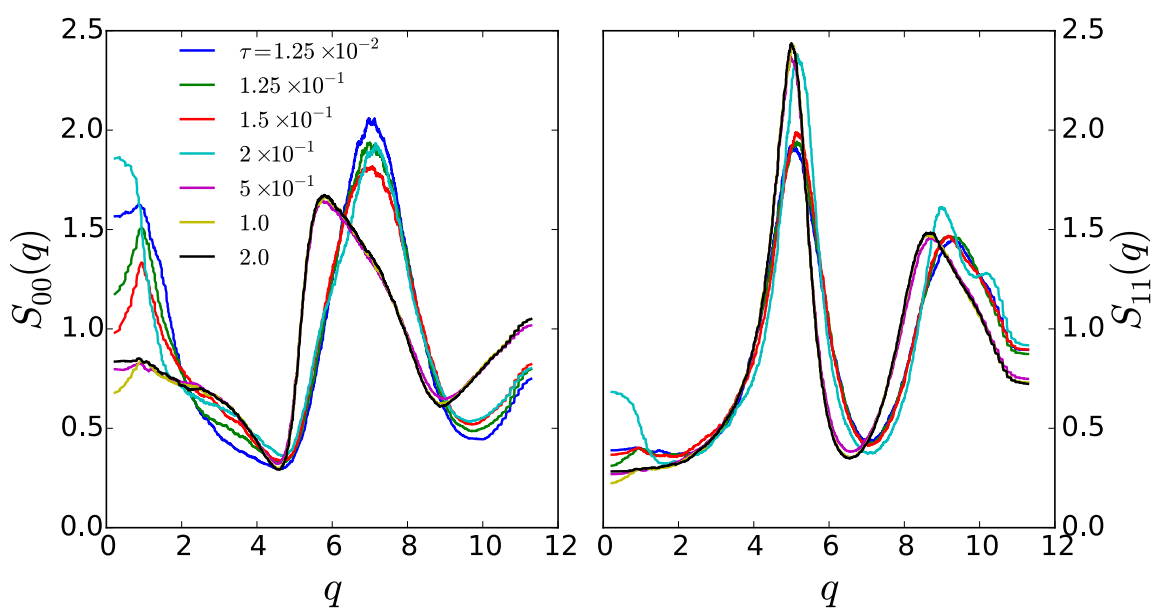

Figure 5.9: Partial structure factors of a system at $u=2 \times 10^{-4}, \phi=0.75$ and $\dot{\gamma}=10^{-6}$. (Left) $\mathrm{S}_{00}$ corresponds to the structures consisting of only small particles, (Right) $S_{11}$ measures the structure factor only for large particles.

Values of $q$ at which $S(q)$ exhibits peaks, correspond to some length scales as $l=2 \pi / q$. Figure 5.10 represents such length scales corresponding to the first two peaks of $S(q) . r_{1}$ is associated with the cluster size and $r_{2}$ indicates the length scale corresponding to the average distance of neighboring particles. While $r_{1}$ reaches zero in the thermal regime due to the lack of clusters, $r_{2}$ reaches a constant value: $r_{2} \approx 1.19$ for $\tau>2 \times 10^{-1}$. This reveals that in the thermal regime, most of the neighbors of each particle of one type are from the other particle type which confirms that $r_{2}$ is close to $R_{0}+R_{1}=1.2$. This argument is also consistent with the assumption that at high temperature, the system is more mixed.

\subsubsection{Intermediate Scattering Function}

The structure factor $S(q)$ measures the spatial correlation between particles but does not contain any information about the temporal correlation. In order to obtain temporal information we need to look at dynamical observables. One possible quantity, relevant to neutronscattering experiments, is the intermediate scattering function,

$$
F(q, t)=\frac{1}{N}\left\langle\rho_{q}(t) \rho_{-q}(0)\right\rangle .
$$




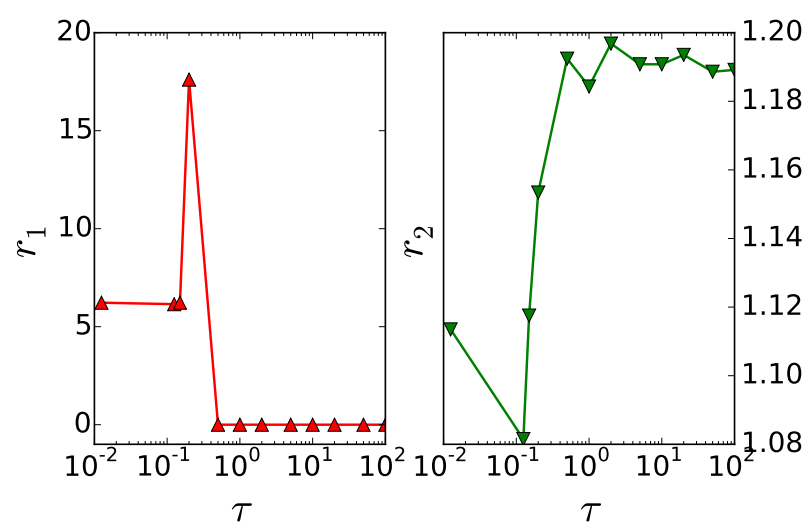

Figure 5.10: The corresponding length-scales of peaks in the structure factor $S(q) . r_{1}$ represents the peak in $S(q)$ when $q<2$,associated with micro-phase separation at small $\tau$. $r_{2}$ corresponds to the largest peak of $S(q)$ at $q \approx 2 \pi$.

In this work, since strain is linearly related to time as $\gamma=t \dot{\gamma}$, we use $F(q, \gamma)$ as the intermediate scattering function. Also note that by definition we have $F(q, 0)=S(q)$.

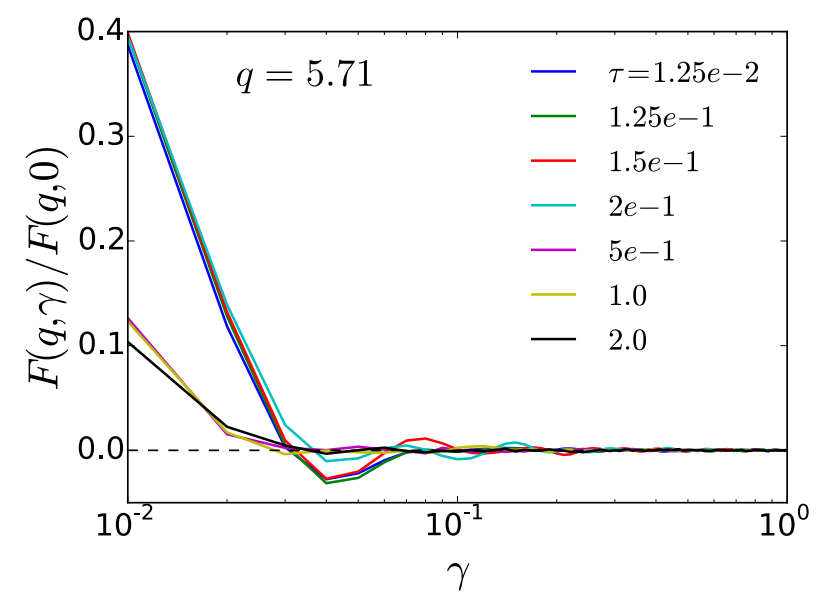

Figure 5.11: The intermediate scattering function $F(q, \gamma)$ for a system at $u=2 \times 10^{-4}, \phi=0.75$ and different $\tau$. We take $q=5.71$ corresponding to the large peak in the $S(q)$.

Figure 5.11 displays $F(q, \gamma) / F(q, 0)$ for $q=5.71$, where the structure factor has the largest peak, at different temperatures. One can observe distinct relaxations of $F(q, \gamma)$ in different thermal regimes. At small $\tau, F(q, \gamma)$ displays oscillations in short times, before getting damped completely. At short times, a particle is "trapped" within its neighbors due to weak attractive forces. When it is disturbed from equilibrium as more strain is imposed to the system, it will initially vibrate within the surrounding structure. However, at longer times, shear and motion of particles lead to the breakage of attractive bonds and rearrangement of the structure, allowing the particle to move 
away from its initial position, which damps the oscillations in $F(q, \gamma)$. On the other hand in the thermal regime, there is no relevant attractive force when thermal fluctuations are dominant. Thus, particles are not confined in local structures and can easily move. In this case, the correlation function $\mathrm{F}(q, \gamma)$ decays faster, with no oscillations.

\subsection{PARTICLE DYNAMICS}

Not only the structure of particle clusters, but also their dynamics is affected by thermal fluctuations. Similar to Chapter 3, we investigate the particles' non-affine displacements, which correct for the convective (affine) part of the particle motion, induced by the average flow field. At any time $t$, the non-affine position of a particle can be written as:

$$
\mathbf{r}_{\text {naff. }}(\mathrm{t})=\mathbf{r}(\mathrm{t})-\hat{\mathbf{x}} \int_{0}^{\mathrm{t}} \mathrm{y}\left(\mathrm{t}^{\prime}\right) \dot{\gamma} \mathrm{dt^{ \prime }}
$$

where $\mathbf{r}(t)=x(t) \hat{\mathbf{x}}+y(t) \hat{\mathbf{y}}$ is the position of particle at time $t$. The second part corresponds to the affine contribution, where $\hat{x}$ and $\hat{y}$ represent unit vectors in shearing and gradient direction respectively. Using these non-affine positions, we compute the mean squared displacement (MSD) of particles. In Figure 5.12, we show the corresponding MSD for $u=2 \times 10^{-4}, \phi=0.75$ (panel (a) and (b)) and $\phi=0.82$ (panel (c) and (d)). In each panel, MSD is calculated for several values of $\tau$, displaying the effect of thermal fluctuation on the dynamics of particles.

Panels (a) and (b) exhibit MSD for a system below $\phi_{\mathrm{G}}(\phi=0.75)$. For such a system, close to the yielding point $\left(\dot{\gamma}=10^{-6}\right)$ and with small $\tau$, attraction leads to a super-diffusive regime in short times $(\gamma)$. At larger strain rates $\left(\dot{\gamma}=10^{-3}\right)$ since repulsive forces dominate, the ballistic regime appears at short time. The diffusive regime is observed in long times $(\gamma)$ regardless of $\dot{\gamma}$.

However in the thermal regime MSD calculation reveals different dynamics of particles. At small strain rates $\left(\dot{\gamma}=10^{-6}\right)$, the system experiences the diffusive dynamics in the whole range of $\gamma$ we investigated. Ballistic regime disappears due the large number of collisions even at small $\gamma$. On the other hand, thermal systems at large $\dot{\gamma}$ show a typical ballistic (short times) and diffusive (long times) behavior. The shearing time-scale becomes much smaller than the thermal time-scale, leading to the suppression of thermal fluctuations. Therefore the ballistic behavior can be observed at small $\dot{\gamma}$.

Panels (c) and (d) in Figure 5.12 illustrate the MSD for a system above $\phi_{\mathrm{G}}$. Close to the yielding point $\left(\dot{\gamma}=10^{-6}\right)$ and at small $\tau$, system displays the same dynamics as the one below $\phi_{\mathrm{G}}$. The superdiffusion is observed at short times which eventually crosses over to the diffusive regime at longer times. There we also found anisotropic 


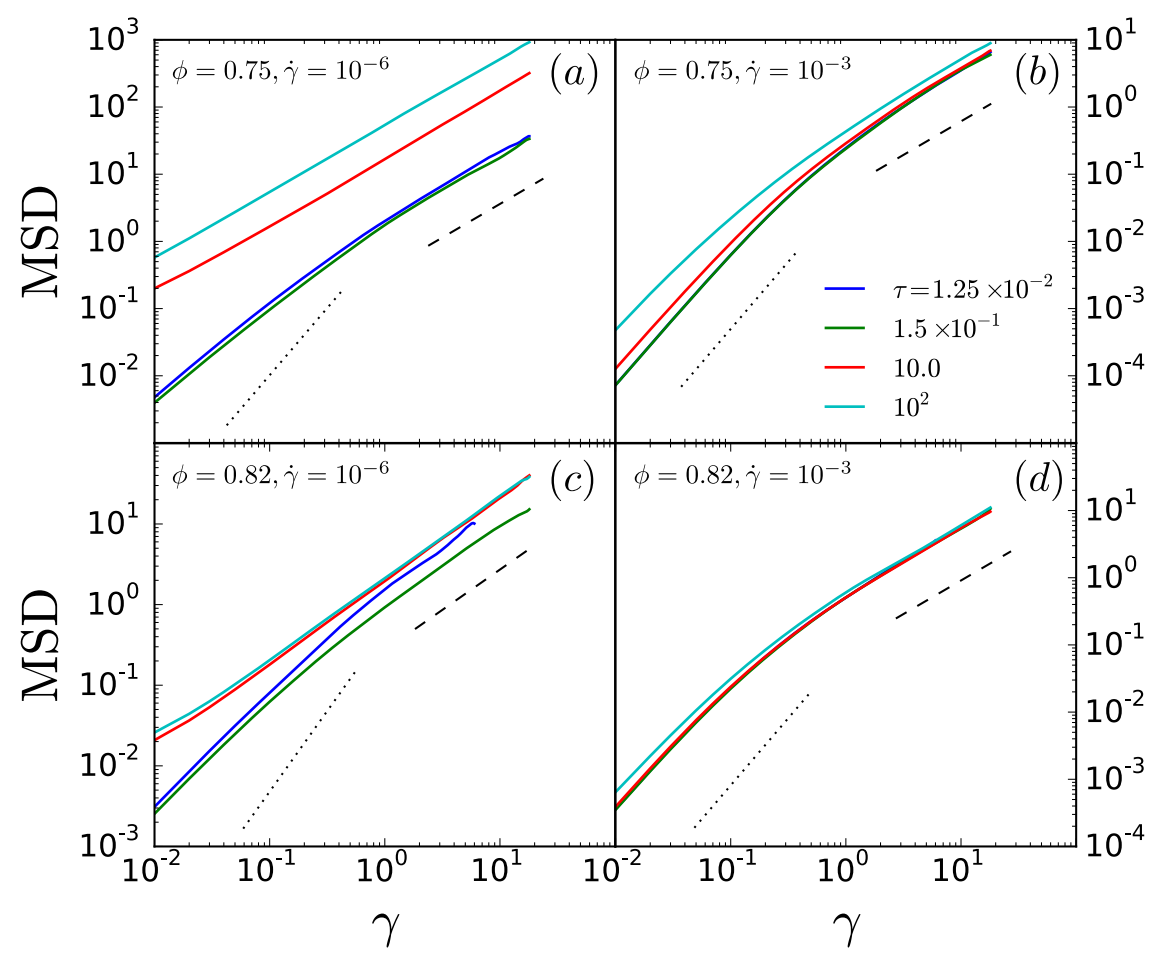

Figure 5.12: non-affine mean-squared displacement for where the system is at (a) $\phi=0.75$ and close to the yield regime, $\dot{\gamma}=10^{-6}$, (b) at $\phi=0.75$ and in the repulsion-dominated regime, $\dot{\gamma}=10^{-3}$, (c) at $\phi=0.82$ above $\phi_{\mathrm{G}}$ and close to the yield regime $\dot{\gamma}=10^{-6}$ and (d) at $\phi=0.82$ and in the fast shearing regime, $\dot{\gamma}=10^{-3}$. The attraction range is set to $u=2 \times 10^{-4}$. The dashed line represent the diffusive behavior, MSD $\propto \gamma^{1}$ while the dotted line indicates the ballistic behavior, $\operatorname{MSD} \propto \gamma^{2}$.

behavior in long times similar to what we observed in Chapter 3 . However in the thermal regime, sub-diffusion is observed in short times, reaching the diffusive regime in long times. Figure 5.13 displays such a transition in MSD for different $\tau$. The appearance of the sub-diffusion can be understood by noting that in the thermal regime and above $\phi_{\mathrm{G}}$, glassy dynamics is dominant. Such a transition in MSD is a well-known feature of sheared hard sphere glasses [Eisenmann et al., 2010]. At large strain rates $\left(\dot{\gamma}=10^{-3}\right)$, fast shearing induces the ballistic dynamics in short times which ends to the diffusion in longer times.

To further investigate the nature of the dynamics under shear, in the presence of both attraction and thermal fluctuations, we also measured the mean squared non-affine velocity and the diffusion coefficients for different strain rates. Figure 5.14 shows the mean-squared velocity for a system with $u=2 \times 10^{-4}, \phi=0.75$ and at different $\tau$. The strain rate is rescaled by the thermal time-scale $t_{T}$. In order to compare the mean squared non-affine velocity of systems at different strain rates, we consider the rescaled quantity $\left(v^{2} / \dot{\gamma}\right)^{2}$. At small 


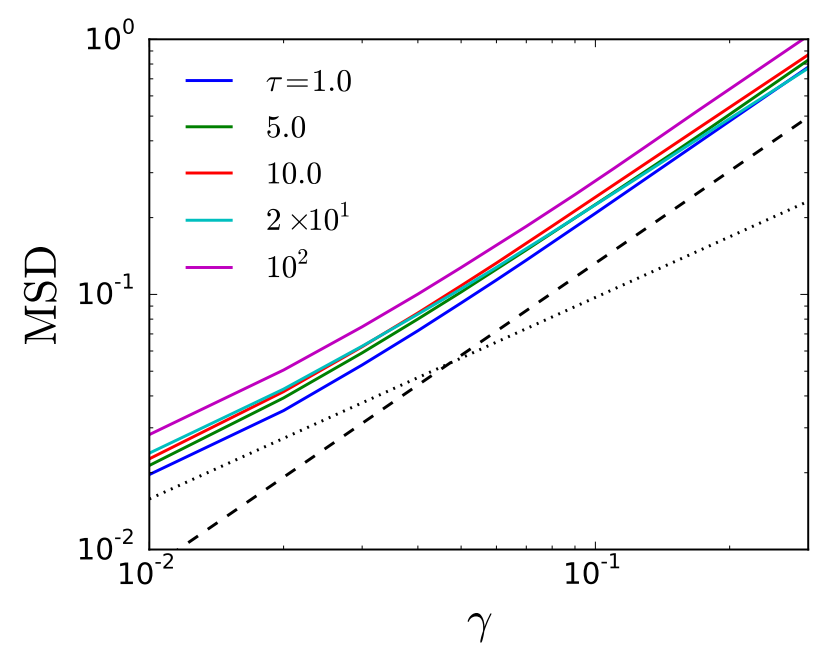

Figure 5.13: MSD in small strains (short times), for a system presented in Figure 5.12, panel (c). Different colors indicate different temperatures, all set the system in the thermal regime $(\tau 1.0)$. at small $\gamma$, a transition from a sub-diffusive to diffusive (or weak superdiffusive) regime takes place, hinting a cage dynamics at small $\gamma$ for systems with $\phi>\phi_{\mathrm{G}}$, in the thermal regime. The dotted line represents MSD $\sim \gamma^{0.79}$, while the dashed line corresponds to $\mathrm{MSD} \sim \gamma^{1.2}$.

$\tau$, this quantity follows the similar dependence on the strain rate as the athermal system plotted in Figure 3.25. For athermal systems we found $\left\langle v^{2}\right\rangle \propto \dot{\gamma}^{1.0}$ in both attractive and repulsive regimes with a kink at the transition. On the other hand at the limit of large $\tau$, we observed $\left\langle(v / \dot{\gamma})^{2}\right\rangle \propto\left(\dot{\gamma} t_{T}\right)^{-2}$. Using Equation 5.4 we conclude that

$$
\left\langle v^{2}\right\rangle \propto \frac{K_{B} T}{m} ;
$$

the squared velocity, indicating the dynamics of particles in short times, is mainly determined by the temperature. At the limit of fast shearing we found $v^{2} \sim \dot{\gamma}^{2}$, fast shearing is the dominant mechanism of particles dynamics, in both athermal and thermal regimes.

Similar to athermal systems (see Figure 3.23), the diffusion coefficient, D, measured in the gradient direction and thus quantifying the extent of non-affine motion over long time, also acquires a higher value at smaller shear-rates. However, Figure 5.15 reveals that in contrast to athermal systems, there is no plateau for D in the limit of small strain rates in thermal regime. The smaller the strain rate, the more dominant non-affine motion over affine motion gets, implying that the latter is more energetically costly. 


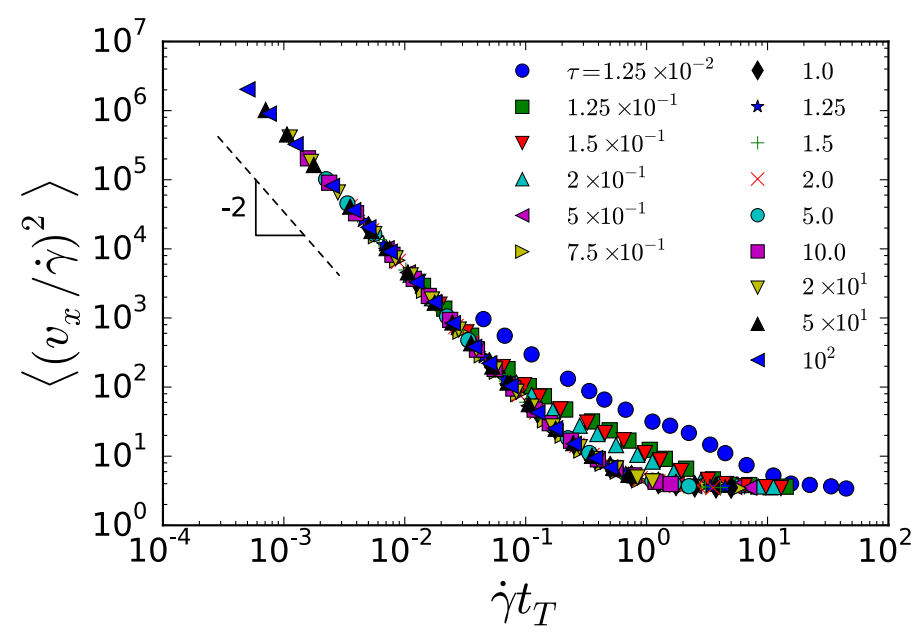

Figure 5.14: Variation of non-affine mean-squared velocity with strain rate (scaled by the thermal time-scale) in shearing direction for a system at $\phi=0.75$ and different $\tau$. The attraction range is set to $u=2 \times 10^{-4}$.

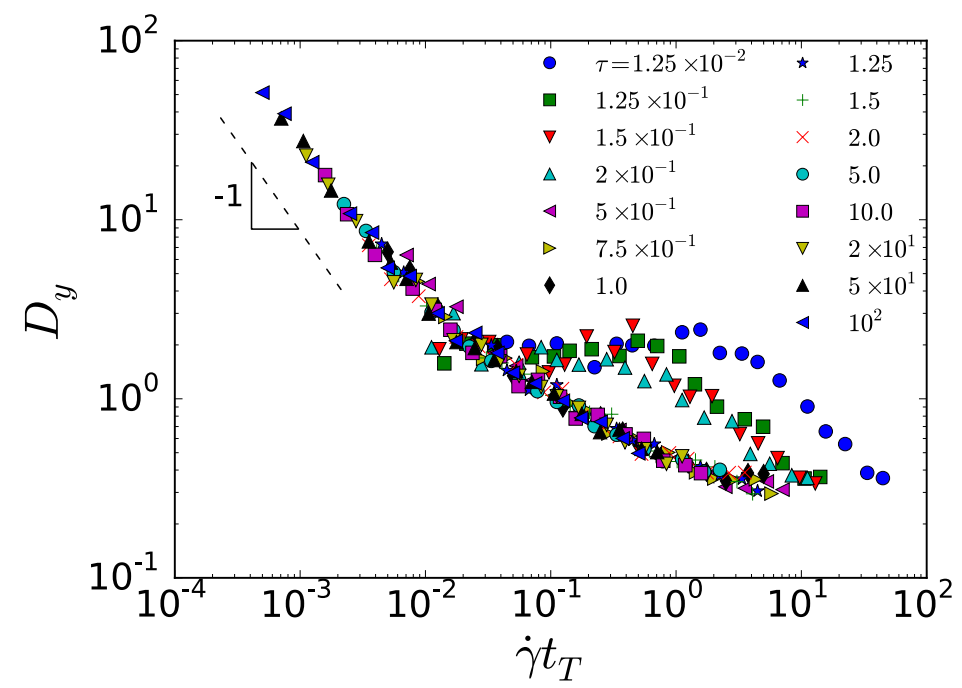

Figure 5.15: The diffusion constant, measured along the gradient direction, as a function of scaled shear-rate, for different $\tau$. The system is at $u=2 \times 10^{-4}$ and $\phi=0.75$. 
$(a)$

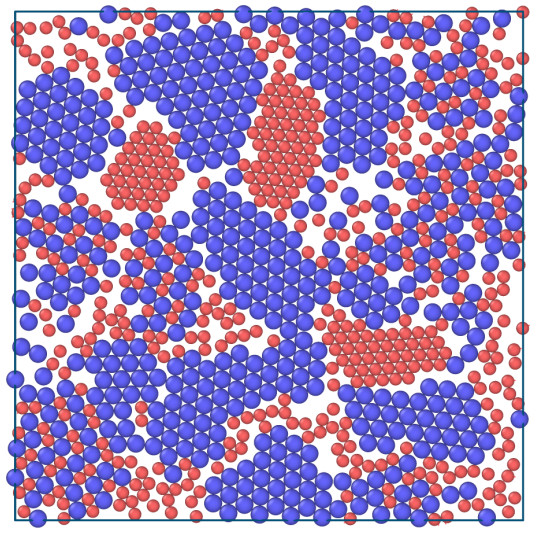

(b)

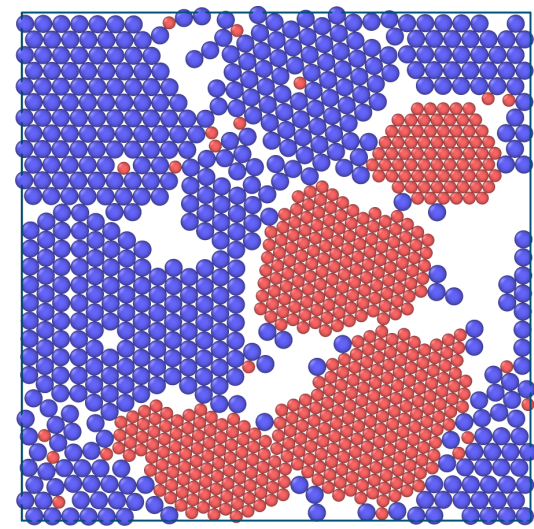

Figure 5.16: Snapshots of the system exhibiting phase separation at $u=2 \times$ $10^{-4}, \phi=0.75$ and $\tau=2 \times 10^{-1}$ (a) without shearing $\dot{\gamma}=0$ and (b) with a finite strain rate $\dot{\gamma}=10^{-6}$.

\subsection{PHASE SEPARATION IN THE PRESENCE OF TEMPERA- TURE}

Phase separation is a quite common phenomena in granular systems. Granular convection which is the size separation of particles induced by shaking the system is a famous example of that [Knight, Jaeger, and Nagel, 1993]. Another type of phase separation occurs in thermal systems, when thermal fluctuations help the system to escape from local minimums in the energy space and settle down in deeper minimum of the energy landscape, corresponding to the phase separated configuration. This kind of thermally-driven phase separations are well studied in many systems, using Cahn-Hillard equations [Cahn and Hilliard, 1958].

At zero temperature, as we have already reported in Chapter 3, weak attractive interactions result in the formation of clusters with an average size of several particles. Those meso-clusters are associated with the first small peak of $S(q)$, which disappears by increasing the strain rate (see Figure 3.19). However, we did not observe any significant growth of those clusters in the simulation time. No phase separation is observed in our athermal systems.

The small peak in $S(q)$ can be also observed in Figure 5.8, for $\tau<=2 \times 10^{-1}$. The corresponding length-scale $r_{1}$, plotted in Figure 5.10, reveals that for some values of $\tau$, thermal fluctuations enhance the cluster formation. More interestingly, we observed that these clusters grow in time as one continues shearing the system, leading to the phase separated configuration of particles. Figure 5.16(b) exhibits such a de-mixed state of a system at $\tau=2 \times 10^{-1}$ with $u=2 \times 10^{-4}, \phi=0.75$ and $\dot{\gamma}=10^{-6}$ after shearing the system for $\gamma=90$. We also simulate a system at zero strain rate, $\dot{\gamma}=0$ and 
$(a)$

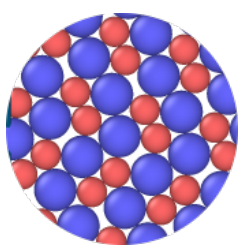

(b)

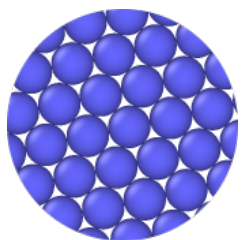

$(c)$

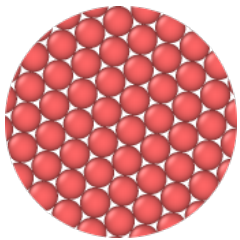

Figure 5.17: Possible structure of phases for a system displaying phase separation. A phase consists of (a) two particle types, (b) just large particles and (c) just small particles. While all these phases are observed in the phase-separated system at $\dot{\gamma}=0$, shearing the system with a finite rate allows only the formation of phases (b) and (c).

observed again the de-mixing of the system via growing clusters.At $\dot{\gamma}=0$, there is an extra phase of the system, the stable cluster of particles from different types. Figure 5.16(a) displays a snapshot of such system. Figure 5.17 shows all kinds of stable clusters in our phase separated systems. However, cluster type (a) is just observed in systems with no shear. It can be rationalized by noting the fact that in contrast to clusters type (b) and (c), clusters type (a) are not completely packed therefore they are more likely to be broken due to shearing.

A valid question is how temperature $(\tau)$ and shearing $(\dot{\gamma})$ affect the phase separation of the system. Whether they collaborate, or compete to induce phase separation in the system. In order to answer this question, we performed simulations at many different $\tau$ and $\dot{\gamma}$.

We already know that increasing $\tau$ has a non-monotonic effect on the phase separation: no local and ordered structures can be found in the thermal regime $\left(\tau>2 \times 10^{-4}\right)$. We also know shearing helps the system to move toward deeper minimums in the energy landscape which can be assumed to be an ordered state of the system. On the other hand, larger strain rate can also reduce the effect of attraction, which is necessary for particles to form clusters.

\subsubsection{Order Parameter: Number of clustered particles}

To study phase separation in our shearing system, the first step is to distinguish particles that are part of the crystallite solid clusters from others. In order to do that, one needs to quantify the structure of clusters. The usual order parameter to do so, is the Steinhardt order parameter, defined as [Steinhardt, Nelson, and Ronchetti, 1983]

$$
Q_{l}(i)=\sqrt{\frac{4 \pi}{2 l+1} \sum_{m=-l}^{m=l}\left|q_{l m(i)}\right|^{2}},
$$


where the complex vector $q_{l m}(i)$ is defined for the ith particle in terms of spherical harmonics as

$$
q_{l m}(i)=\frac{1}{N_{b}(i)} \sum_{j=i}^{N_{b}(i)} Y_{l m}\left(\vec{r}_{i j}\right) .
$$

$N_{b}(i)$ is the number of neighbors of particle $i$ and $\vec{r}_{i j}$ is the connecting vector between particles $i$ and $j$.

Howeve, we observed that different phases have a unique and ordered structure in our systems. Figure 5.17 displays possible clusters which are stable in the system we study. $\mathrm{Q}_{6}$ is known to be a proper choice of order parameter to distinguish such hexagonal structures. However, because of the unique symmetry of all stable clusters in our simulations, we choose another order parameter that captures the same information as $Q_{6}$, but is more effective and faster to calculate. This order parameter, which we use to measure the amount of phase separation, is defined as the number of clustered particles divided by the total number particles,

$$
\mathrm{N}^{*}=\frac{\mathrm{N}_{\text {clustered }}}{\mathrm{N}}
$$

A particle belongs to an arbitrary cluster if and only if it has exactly six neighbors of the same type as itself, or has a neighbor with this condition. In the algorithm we use, overlapping clusters are considered to be parts of a larger cluster. The total $\mathrm{N}_{\text {clustered }}$ is then obtained by adding the number of clustered particles from each type.

Investigating the dynamics of $\mathrm{N}^{*}$ in time, provides us with information about the amount and also the rate of phase separation in the system. The left panel of Figure 5.18 demonstrates $N^{*}(\gamma)$ for a system at a fixed temperature $\tau=2 \times 10^{-1}$ and different strain rates. One can see at $\tau=2 \times 10^{-1}$, phase separation is faster and more pronounced in the regime of small strain rates. After shearing the system for $\gamma=40$, while $\dot{\gamma}=10^{-6}$, more than $80 \%$ of particles are in separated phases, $\mathrm{N}^{*}<0.6$ at other strain rates. Interestingly, Increasing $\dot{\gamma}$ does not always decrease $\mathrm{N}^{*}$. After some transient time, $\mathrm{N}^{*}\left(\dot{\gamma}=10^{-5}\right)>\mathrm{N}^{*}\left(\dot{\gamma}=7.5 \times 10^{-6}\right)$. The right panel in Figure 5.18 displays $\mathrm{N}^{*}(\gamma)$ for systems at different temperature but all with a similar strain rate, $\dot{\gamma}=10^{-6}$. The phase separation is faster in $\tau=2 \times 10^{-1}$ while the system at $\tau=1.25 \times 10^{-2}$ seems to behave like an athermal system: no growth in cluster or a very slow growth rate.

\subsubsection{Phase-separation Parameter Space}

In order to find regions of the parameter space at which temperature and shearing collaborate to push the system toward phase separation and regions they compete, we need to obtain $\mathrm{N}^{*}$ at different $\tau$ and $\dot{\gamma}$. 

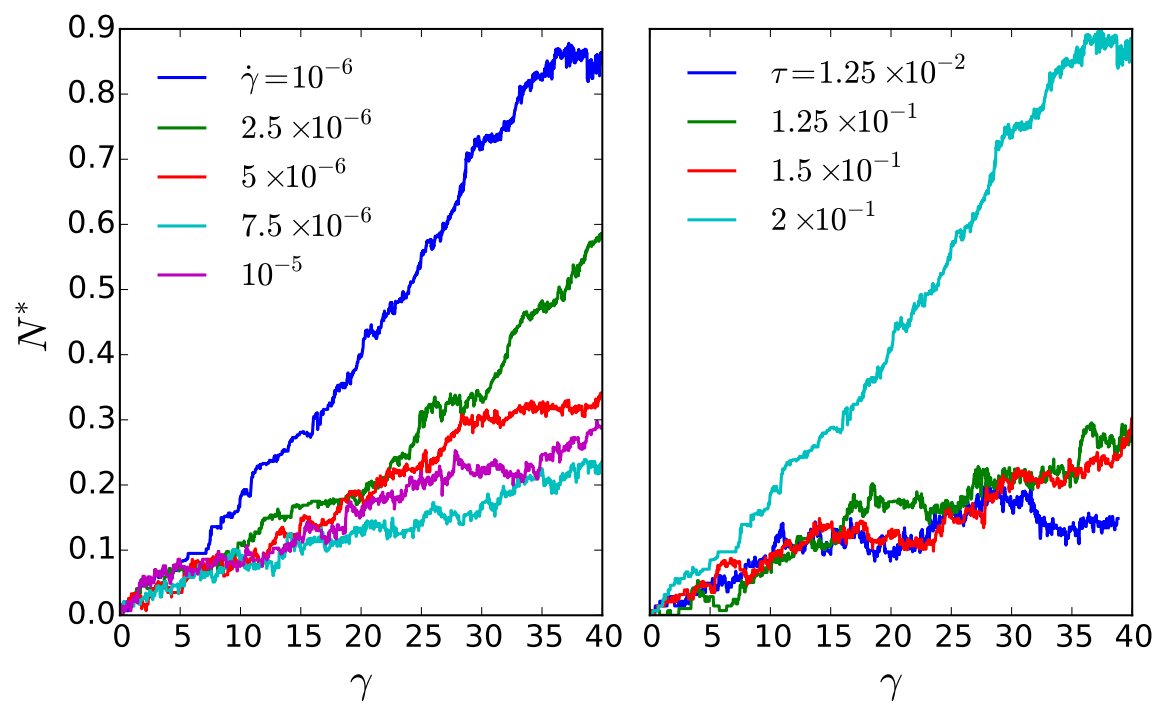

Figure 5.18: Number of particles in packed clusters as a function of strain $\gamma$. Data is plotted for systems at (left) tau $=2 \times 10^{-1}$ and different $\dot{\gamma}$, (right) $\dot{\gamma}=10^{-6}$ and different $\tau$. The attraction range is set to $u=2 \times 10^{-4}$ and the volume fraction is $\phi=0.75$.

As a measure of phase separation, we calculated $\mathrm{N}^{*}$ averaged over a strain window of size $\Delta \gamma=5.0$, after shearing the system for $\gamma=35$ at small strain rates $\left(\dot{\gamma}<=10^{-5}\right)$ and $\gamma=15$ at large strain rates $\left(\dot{\gamma}>10^{-5}\right)$. Figure 5.19 visualize $\mathrm{N}^{*}$ versus both $\tau$ and $\dot{\gamma}$, for a system at $u=2 \times 10^{-4}$ and $\phi=0.75$.

In the regime of small strain rates, even in the athermal regime, more than 10 percent of particles are arranged in ordered clusters. Increasing $\tau$ leads to faster growth and larger value of $\mathrm{N}^{*}$, which eventually reaches $N^{*}=0.9$ at $\tau=2 \times 10^{-1}$. Further increase of $\tau$ sets the system in the thermal regime $\left(\tau>2 \times 10^{-1}\right)$, where thermal fluctuations overcome attractive force, contacts and local structures are destroyed and consequently, $\mathrm{N}^{*}=0$.

If we look at the behavior of the system at $\tau \leqslant 1.5 \times 10^{-2}$, there is a range of strain rates at which $\mathrm{N}^{*}(\dot{\gamma})$ displays a non-monotonic behavior. At small $\dot{\gamma}$, attractive interactions are dominant, shearing can not rupture the local structure of particles but helps the system to explore the energy landscape and find deeper local minimums corresponding to more ordered and phase-separated configurations. On the other hand, large $\dot{\gamma}$ sets the system in repulsion-dominated regime, where shearing overcomes attraction and the system flows as a repulsive fluid. Since attraction plays a key role in phase-separation, it is reasonable to find $\mathrm{N}^{*}(\dot{\gamma})$ as a decreasing function of $\dot{\gamma}$ for strain rates larger than a threshold. We observed that $\mathrm{N}^{*}$ starts to decrease at roughly the same $\dot{\gamma}$ at which athermal $\sigma(\dot{\gamma})$ also exhibits a fast drop. However, the non-monotonic behavior of $\mathrm{N}^{*}(\dot{\gamma})$ at intermediate strain rates, hints that a more complicated interplay of attraction, shearing 


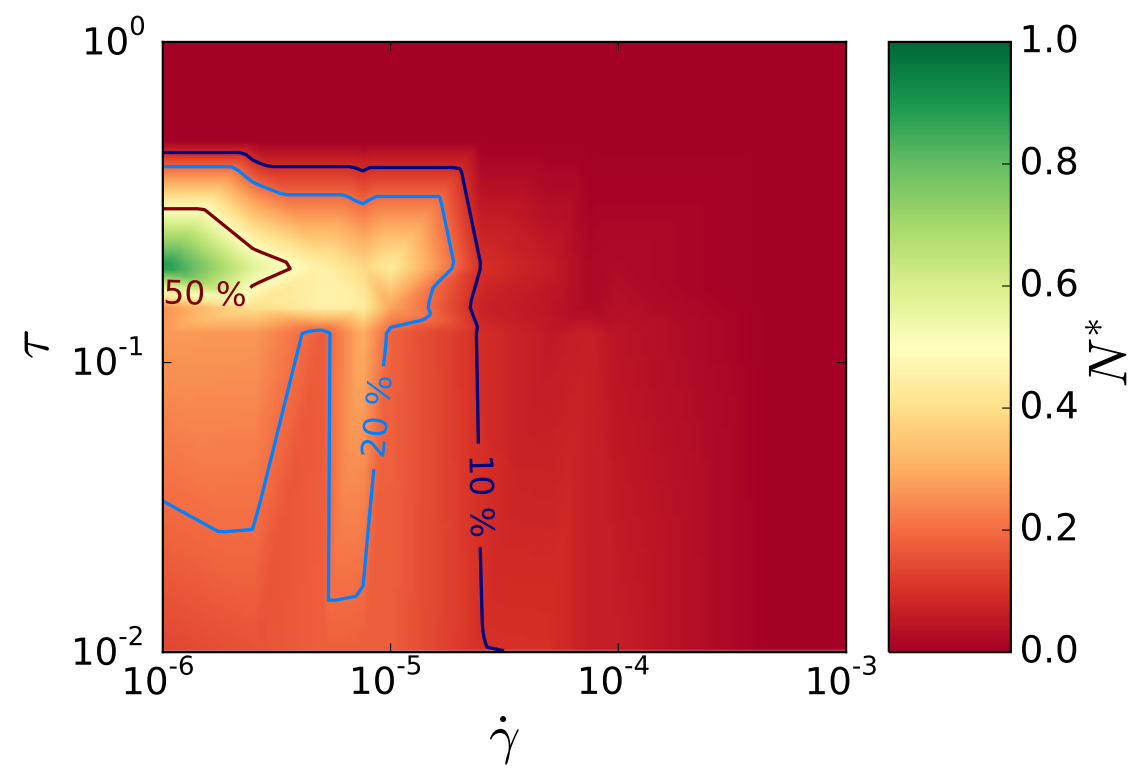

Figure 5.19: The amount of phase-separation for an attractive system at $u=$ $2 \times 10^{-4}$ and $\phi=0.75$. The number of particles in separated phases, $\mathrm{N}^{*}$, is plotted at different values of temperature $\tau$ and strain rate $\dot{\gamma}$.

and temperature is involved. More studies are needed to understand the origin and underlying mechanisms of such a behavior.

\subsection{DIFFERENT DISSIPATION MODELS: PRELIMINARY RESULTS}

In this Chapter so far, we have used $\mathrm{CD}_{n}$ dissipation model. Other possibilities as discussed in Chapter 4 , are using CD and RD models, and turning on the thermal fluctuations. In any case, the fluctuationdissipation relation, Equation 5.2, needs to be satisfied.

Figure 5.20 demonstrates flow curves of attractive systems at $u=$ $2 \times 10^{-} 5, \phi=0.75$ and with CD model, at different values of temperature $\tau$. The right panel represents the over-damped regime, where $\mathrm{b}=2.0$ while the left panel displays the under-damped regime with $\mathrm{b}=0.05$. We observed in the over-damped regime, the system is sensitive to thermal fluctuations only at very low strain rates, where increasing the temprerature to $\tau=2 \times 10^{-4}$, destroys the low strain rate behavior of such flow curves, $\sigma \sim \dot{\gamma}^{\alpha}$ with $\alpha<1.0$, and gradually governs the viscous behavior, $\sigma \sim \dot{\gamma}$. Increasing $\tau$ further, will destroy the contacts and structure of the system, leading to large fluctuations in the measured stress. More simulations and samplings are needed to study the behavior of the system at $\tau \geqslant 5 \times 10^{-1}$.

The more interesting case is the under-damped regime, where temperature affects flow curves in a wider range of strain rates, including the non-monotonic part. Using Pećlet number $P_{e}$ and rescaled stress $\sigma / \mathrm{T}$, the inertial branch of flow curves follow a master curve. Increas- 

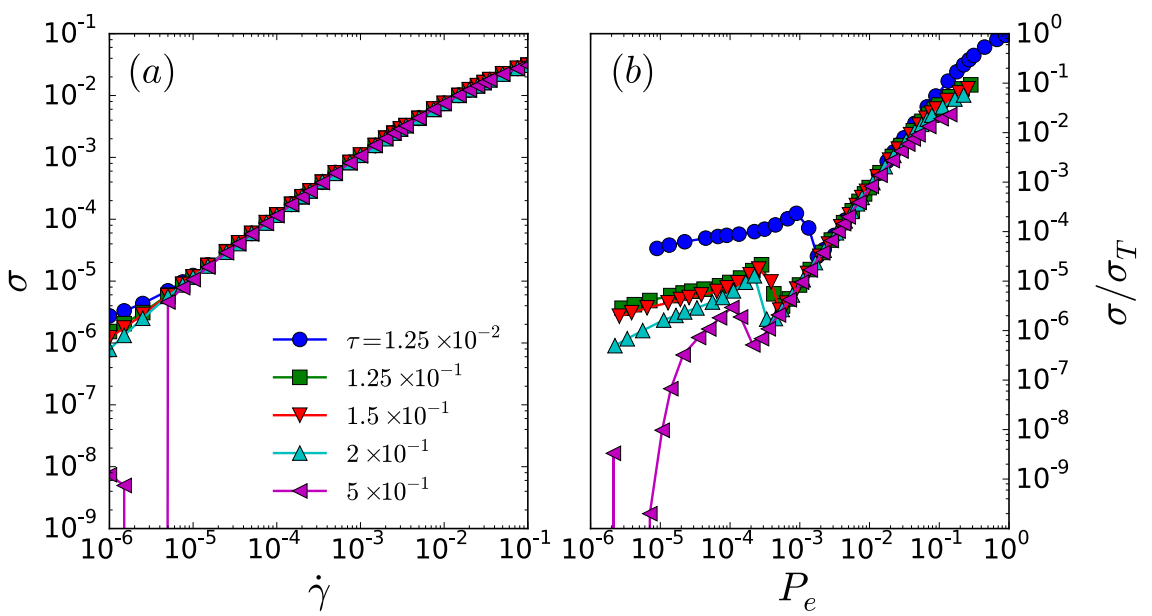

Figure 5.20: Flow curves of systems with $C D$ dissipation model at $u=2 \times$ $10^{-5}, \phi=0.75$, (a) $b=2.0$ and (b) $b=0.05$. In the underdamped regime, data is represented in terms of rescaled strain rate, Pećlet number, and rescaled stress $\sigma / \sigma_{\mathrm{T}}$.

ing $\tau$ alters the low strain rates regime, where the growth of $\sigma$ with increasing $\mathrm{P}_{\mathrm{e}}$ becomes faster. More interesting is the effect of thermal fluctuations on shear-banding. Do thermal fluctuations help shearbands to form, or it ensures the homogeneous flow? More studies are needed to address this question.

To introduce thermal fluctuations in systems with RD model, the Langevin equation governs the dynamics of particles as,

$$
\overrightarrow{\mathrm{F}}_{i}=\sum_{j=1}^{j=z_{i}} \vec{F}_{i j}^{C}+-\zeta(\vec{v}-\dot{\gamma} y \hat{x})+\vec{F}^{R} .
$$

In Equation 5.17, the force applied on particle $i$ is obtained by adding the viscous drag (RD) and random force $F^{R}$ to the contribution from interactions with its $z_{b}$ neighbors. The random force $F^{R}$ is a random number obtained from a Gaussian distribution with correlation function as

$$
\left\langle F_{\alpha}^{R}\left(t_{1}\right) F_{\beta}^{R}\left(t_{2}\right)\right\rangle=2 \zeta K_{B} T \delta_{\alpha \beta} \delta\left(t_{1}-t_{2}\right) .
$$

For such dynamics, Pećlet number is defined as $P_{e}=\dot{\gamma} t_{T}$, while $t_{T}$ is now given by Equation 5.5,

$$
\mathrm{t}_{\mathrm{T}}=\frac{\zeta \mathrm{r}_{\mathrm{c}}^{2}}{\mathrm{~K}_{\mathrm{B}} \mathrm{T}^{\prime}}
$$

Figure 5.21, displays flow curves of systems with Langevin dynamics at $u=2 \times 10^{-5}, \phi=0.75$, for different values of $\tau$ and $\zeta$. The right panel corresponds to $\zeta=2.0$, while the left panel represents results with $\zeta=5 \times 10^{-3}$. In both cases, we observed that flow curves are more sensitive to $\tau$, compared to systems with fluctuations at contacts, 
$C D_{n}$ and $C D$ dissipation models. While in $C D$ and $C D_{n}$ models, thermal regime starts at $\tau>2 \times 10^{-1}$, here increasing the temperature to $\tau \geqslant 1.25 \times 10^{-1}$, completely destroys attraction-induced features of flow curves. Resulting flow curves, reproduce the behavior of repulsive systems discussed in [Ikeda et al., 2013; Kawasaki et al., 2014].

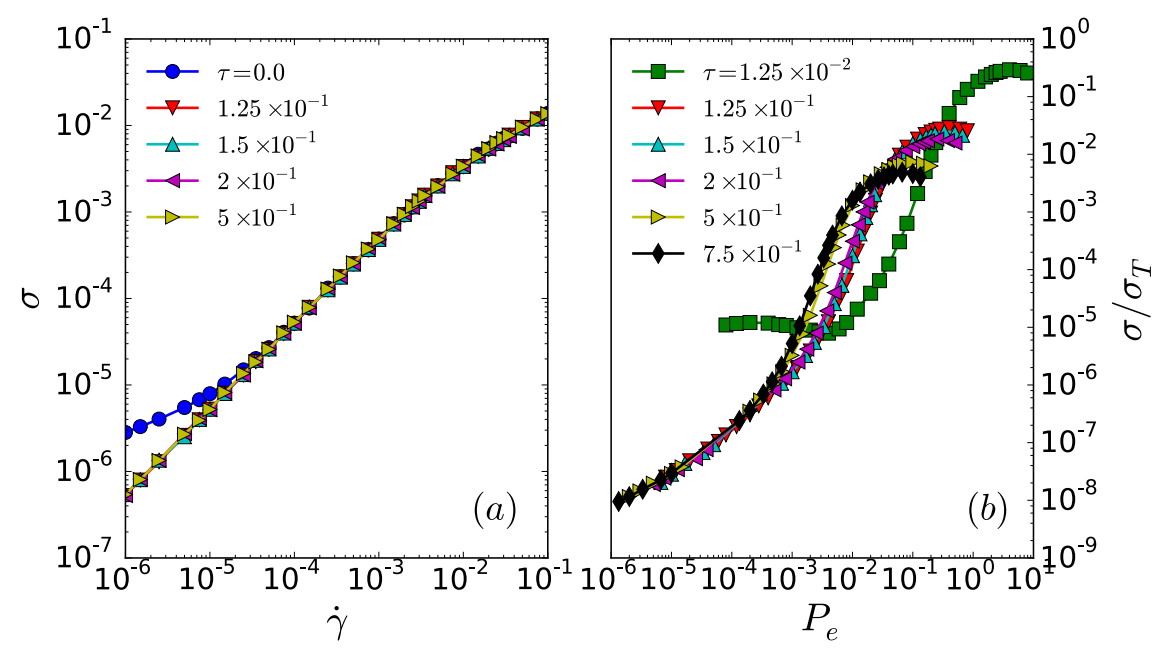

Figure 5.21: Flow curves for systems with RD dissipation model at $u=2 \times$ $10^{-5}, \phi=0.75$, (a) $\zeta=2.0$ and (b) $\zeta=5 \times 10^{-3}$. In panel (b), data is represented in terms of rescaled strain rate, Pećlet number, and rescaled stress $\sigma / \sigma_{\mathrm{T}}$. 

Part III

CLOSING WORDS 



\section{CONCLUSION AND OUTLOOK}

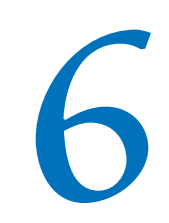

In this thesis, we study the rheology of soft particles, in the presence of weak attractive interactions. We performed molecular dynamics simulations to obtain flow curves of the system, as well as microscopic dynamics of particles. One of the parameters which has a huge effect on rheology, is the distance to jamming point. We vary the volume fraction of the system to capture this dependence.

In Chapter 3, we introduced a minimal interaction potential between particles, Equation 3.1, to model weak attractive interactions. The parameter $u$ is introduced to tune the strength and range of attraction simultaneously. We have found that setting a finite value to $u$, results in a dramatic deviation from the well-studied rheology of repulsive systems, specially in the regime of low strain rates. For instance, A finite yield stress emerges even far below the jamming point. The connectivity of the system, $z$, jumps to values slightly above the isostatic point, which indicates the development of a nearly isostatic network of particles. This system spanning network, is responsible for the weak solid-like behavior below the jamming, at low strain rates. The yield stress of this weak solid, has been expressed in terms of attraction and distance to the isostatic point.

The interplay between the attractive aggregation and shear-induced rupture, leads to the non-monotonic behavior of $\sigma(\dot{\gamma})$ with increasing $\dot{\gamma}$. The non-monotonic flow curve is associated with the mechanical instability and consequently, shear-banding in such systems. We found that in order to observe persistence shear bands, the system size needs to exceed a certain value. After discussing the theoretical concept of shear-banding, we presented a study of shear-bands in simulations. All together, we provided a numerical framework that can be useful to study features of shear bands in attractive systems, in details.

In Chapter 4, we focus on the role of dissipation mechanisms on novel rheology of attractive systems. Several models are introduced: the $\mathrm{CD}_{\mathrm{n}}$ model, in which a drag force proportional to the normal relative velocity of overlapping particles is applied on them. CD model, in which the tangential relative velocity, in addition to the normal component, is dissipated. RD model is also studied briefly, where a viscous drag force is applied on particles whose velocity deviates from the imposed flow velocity. We found that the choice of dissipation model, as they represent different physical systems, has a huge impact on the rheology. Tangential dissipation is observed to elimi- 
nate the non-monotonic behavior of flow curves, in the over-damped regime.

Using CD model, in the under-damped regime $\sigma(\dot{\gamma})$ exhibits a sharp non-monotonic crossover from viscous to inertial flow, as $\dot{\gamma}$ is increased. Investigating this feature, we found shear-banding in the form of coexistence of viscous and inertial flow, with a heterogeneous stress field through the system. We discussed the stability of localized stress, using virial and kinetic contributions to the stress. This kind of shear bands are intrinsically different from shear-bands in systems with $\mathrm{CD}_{\mathrm{n}}$ model. In the former, we found that local volume fraction is heterogeneous as well as stress and local flow rate. Features of these novel shear-bands can be a subject of future studies.

In Chapter 5, we studied the effect of thermal fluctuations on the rheology by exploring a range of temperature, from athermal limit to the glassy regime. Although both athermal and glassy limits are found to display disordered configurations, in the intermediate regime thermal fluctuations drive the system toward a phase-separated ordered state. The structure and dynamics of the system is studied in terms of flow curves, structure factors and particle displacements. We defined a simple order parameter to quantify the phase-separation, as a function of temperature and strain rate. This order parameter provides us with information about regions of the parameter space, in which shearing and thermal fluctuations collaborate or compete with respect to the phase separation. Although the interplay between thermal fluctuations and shearing can be understood in limits of small and large temperature and strain rates, in the intermediate regime a more complicated relation exists. More studies are needed to investigate the underlying physical mechanism responsible for such complicated interplay between thermal effects and shearing. 


\section{BIBLIOGRAPHY}

Bagnold, R. A. (1954). "Experiments on a Gravity-Free Dispersion of Large Solid Spheres in a Newtonian Fluid under Shear." In: Proceedings of the Royal Society of London A: Mathematical, Physical and Engineering Sciences 225.116o, pp. 49-63. ISSN: 0080-4630. DoI: 10.1098/rspa. 1954.0186 (cit. on pp. 15, 30).

Bécu, Lydiane, Sébastien Manneville, and Annie Colin (2006). "Yielding and Flow in Adhesive and Nonadhesive Concentrated Emulsions." In: Phys. Rev. Lett. 96 (13), p. 138302. DoI: 10.1103/PhysRevLett. 96. 138302. URL: http: //link.aps . org/doi/10.1103/PhysRevLett. 96.138302 (cit. on pp. 17, 19).

Berzi, Diego and James T. Jenkins (2015). "Inertial shear bands in granular materials." In: Physics of Fluids 27.3, 033303. Dor: http : / / dx . doi . org / 10 . 1063/1 . 4914920. URL: http : / / scitation . aip.org/content/aip/journal/pof2/27/3/10.1063/1.4914920 (cit. on p. 79).

Bitzek, Erik, Pekka Koskinen, Franz Gähler, Michael Moseler, and Peter Gumbsch (2006). "Structural Relaxation Made Simple." In: Phys. Rev. Lett. 97 (17), p. 170201. DOI: 10.1103/PhysRevLett. 97. 170201. URL: http : // link.aps.org/doi/10 . 1103/PhysRevLett. 97.170201 (cit. on p. 23).

Boyer, Fran çois, Élisabeth Guazzelli, and Olivier Pouliquen (2011). "Unifying Suspension and Granular Rheology." In: Phys. Rev. Lett. 107 (18), p. 188301. DOI: 10.1103/PhysRevLett. 107. 188301. URL: http : / / link. aps . org/doi/10.1103/PhysRevLett . 107 . 188301 (cit. on p. 15).

Brown, Eric and Heinrich M. Jaeger (2012). "The role of dilation and confining stresses in shear thickening of dense suspensions." In: Journal of Rheology 56.4, 875-923. DOI: 10 . 1122/1 . 4709423. URL: http: //scitation.aip.org/content/sor/journal/jor2/56/4/ 10.1122/1.4709423 (cit. on p. 17).

Cahn, John W. and John E. Hilliard (1958). "Free Energy of a Nonuniform System. I. Interfacial Free Energy." In: The Journal of Chemical Physics 28.2, pp. 258-267. Dor: http://dx. doi.org/10.1063/ 1. 1744102. URL: http : / / scitation . aip . org / content / aip / journal/jcp/28/2/10.1063/1.1744102 (cit. on p. 105).

Campbell, Charles S. (2002). "Granular shear flows at the elastic limit." In: Journal of Fluid Mechanics 465, pp. 261-291. IssN: 1469-7645. DOI: 10.1017/S002211200200109X. URL: http: //journals . cambridge. org/article_S002211200200109X (cit. on p. 30).

Castellanos, A. (2005). "The relationship between attractive interparticle forces and bulk behaviour in dry and uncharged fine pow- 
ders." In: Advances in Physics 54.4, pp. 263-376. DoI: 10.1080 / 17461390500402657. eprint: http://dx. doi.org/10.1080/17461390500402657. URL: http : / / dx . doi . org / 10 . 1080/17461390500402657 (cit. on p. 18).

Chaudhuri, Pinaki, Ludovic Berthier, and Srikanth Sastry (2010). "Jamming Transitions in Amorphous Packings of Frictionless Spheres Occur over a Continuous Range of Volume Fractions." In: Phys. Rev. Lett. 104 (16), p. 165701. DoI: 10.1103 / PhysRevLett . 104. 165701. URL: http: / / link. aps . org/doi/10 . 1103/PhysRevLett . 104.165701 (cit. on pp. 12, 13).

Chaudhuri, Pinaki, Ludovic Berthier, and Lydéric Bocquet (2012). "Inhomogeneous shear flows in soft jammed materials with tunable attractive forces." In: Phys. Rev. E 85 (2), p. 021503. DoI: 10.1103/ PhysRevE . 85.021503. URL: http: // link . aps . org/doi/10 . 1103/ PhysRevE.85. 021503 (cit. on pp. 18, 28, 30).

Chen, Daniel T.N., Qi Wen, Paul A. Janmey, John C. Crocker, and Arjun G. Yodh (2010). "Rheology of Soft Materials." In: Annual Review of Condensed Matter Physics 1.1, pp. 301-322. DoI: 10.1146/ annurev - conmatphys - 070909 - 104120. eprint: http : / / dx . doi . org/10 . 1146/annurev - conmatphys - 070909 - 104120. URL: http: // dx . doi . org / 10 . 1146/annurev - conmatphys - 070909 - 104120 (cit. on pp. 9, 13).

Coussot, P. (2007). "Rheophysics of pastes: a review of microscopic modelling approaches." In: Soft Matter 3 (5), pp. 528-540. DOI: 10. 1039/B611021P. URL: http : //dx. doi . org/10 . 1039/B611021P (cit. on pp. 17, 30).

Coussot, P. and G. Ovarlez (2010). "Physical origin of shear-banding in jammed systems." In: The European Physical Journal E 33.3, 183-188. ISSN: 1292-895X. DOI: 10 . 1140/epje/i2010 - 10660 - 9. URL: http : //dx.doi.org/10.1140/epje/i2010-10660- 9 (cit. on p. 19).

D. Olmsted, Peter and David Lu, C-Y. (1999). "Phase coexistence of complex fluids in shear flow." In: Faraday Discuss. 112 (o), pp. 183194. DOI: 10. 1039/A900245F. URL: http://dx. doi.org/10 . 1039/ A900245F (cit. on p. 54).

Denkov, N. D., S. Tcholakova, K. Golemanov, K. P. Ananthapadmanabhan, and A. Lips (2008). "Viscous Friction in Foams and Concentrated Emulsions under Steady Shear." In: Phys. Rev. Lett. 100 (13), p. 138301. DOI: 10.1103 / PhysRevLett . 100.138301. URL: http : / / link . aps . org/doi/10.1103/PhysRevLett. 100. 138301 (cit. on p. 15).

Dhont, Jan K. G. (1999). "A constitutive relation describing the shearbanding transition." In: Phys. Rev. E 6o (4), pp. 4534-4544. DOI: 10.1103/PhysRevE.60.4534. URL: http://link. aps . org/doi/10. 1103/PhysRevE.60. 4534 (cit. on pp. 19, 32, 54, 58, 60).

Divoux, Thibaut, Vincent Grenard, and Sébastien Manneville (2013). "Rheological Hysteresis in Soft Glassy Materials." In: Phys. Rev. 
Lett. 110 (1), p. 018304. DoI: 10.1103/PhysRevLett. 110.018304. URL: http : / / link . aps . org / doi / 10 . 1103/PhysRevLett . 110 . 018304 (cit. on p. 60).

Divoux, Thibaut, Marc A. Fardin, Sebastien Manneville, and Sandra Lerouge (2016). "Shear Banding of Complex Fluids." In: Annual Review of Fluid Mechanics 48.1, 81-103. DoI: 10.1146 / annurev fluid-122414-034416. eprint: http : / / dx . doi .org / 10 . 1146/ annurev - fluid- 122414 - 034416. URL: http : //dx . doi . org/10. 1146/annurev - fluid-122414-034416 (cit. on p. 18).

Durian, D. J. (1995). "Foam Mechanics at the Bubble Scale." In: Phys. Rev. Lett. 75 (26), pp. 4780-4783. DoI: 10.1103/PhysRevLett. 75. 4780. URL: http: //link. aps . org/doi/10.1103/PhysRevLett. 75. 4780 (cit. on pp. 12, 15).

- (1997). "Bubble-scale model of foam mechanics:mMelting, nonlinear behavior, and avalanches." In: Phys. Rev. E 55 (2), pp. 17391751. DOI: 10 . 1103/PhysRevE . 55 . 1739. URL: http : // link . aps . org/doi/10.1103/PhysRevE.55.1739 (cit. on pp. 66, 67).

Eckert, T. and E. Bartsch (2002). "Re-entrant Glass Transition in a Colloid-Polymer Mixture with Depletion Attractions." In: Phys. Rev. Lett. 89 (12), p. 125701. DoI: 10.1103/PhysRevLett.89.125701. URL: http : / / link . aps . org / doi / 10 . 1103 / PhysRevLett . 89 . 125701 (cit. on p. 17).

Eisenmann, Christoph, Chanjoong Kim, Johan Mattsson, and David A. Weitz (2010). "Shear Melting of a Colloidal Glass." In: Phys. Rev. Lett. 104 (3), p. 035502. DOI: 10.1103 / PhysRevLett. 104. 035502. URL: http://link.aps .org/doi/10.1103/PhysRevLett. 104.035502 (cit. on p. 102).

Fall, Abdoulaye, Anaël Lemaître, Fran çois Bertrand, Daniel Bonn, and Guillaume Ovarlez (2010). "Shear Thickening and Migration in Granular Suspensions." In: Phys. Rev. Lett. 105 (26), p. 268303. DOI: 10.1103/PhysRevLett . 105 . 268303. URL: http : / / link . aps . org/doi/10.1103/PhysRevLett. 105.268303 (cit. on p. 84).

Fielding, S M (2014). "Shear banding in soft glassy materials." In: Reports on Progress in Physics 77.10, p. 102601. URL: http: //stacks . iop. org/0034-4885/77/i=10/a=102601 (cit. on p. 53).

Forterre, Yoël and Olivier Pouliquen (2008). "Flows of Dense Granular Media." In: Annual Review of Fluid Mechanics 40.1, pp. 1-24. DOI: 10 . 1146/annurev . fluid . 40 . 111406 . 102142. eprint: http : //dx.doi.org/10.1146/annurev. fluid.40.111406.102142. URL: http://dx.doi.org/10.1146/annurev. fluid.40.111406.102142 (cit. on p. 10).

Fraysse, N., H. Thomé, and L. Petit (1999). "Humidity effects on the stability of a sandpile." In: The European Physical Journal B - Condensed Matter and Complex Systems 11.4, pp. 615-619. IssN: 14346036. DOI: 10 . 1007/s100510051189. URL: http://dx. doi . org/10. 1007/s100510051189 (cit. on p. 18). 
Grob, Matthias, Claus Heussinger, and Annette Zippelius (2014). "Jamming of frictional particles: A nonequilibrium first-order phase transition." In: Phys. Rev. E 89 (5), p. 050201. DOI: 10.1103/PhysRevE. 89. 050201. URL: http: // link. aps.org/doi/10 . 1103/PhysRevE. 89.050201 (cit. on pp. 16, 17).

Grob, Matthias, Annette Zippelius, and Claus Heussinger (2016). "Rheological chaos of frictional grains." In: Phys. Rev. E 93 (3), p. 030901. DOI: 10.1103/PhysRevE.93.030901. URL: http://link.aps.org/ doi/10.1103/PhysRevE. 93.030901 (cit. on p. 16).

Gu, Yile, Sebastian Chialvo, and Sankaran Sundaresan (2014). "Rheology of cohesive granular materials across multiple dense-flow regimes." In: Phys. Rev. E 90 (3), p. 032206. Dor: 10. 1103/PhysRevE. 90.032206. URL: http: // link. aps .org/doi/10.1103/PhysRevE. 90.032206 (cit. on p. 42).

Hecke, M van (2010). "Jamming of soft particles: geometry, mechanics, scaling and isostaticity." In: Journal of Physics: Condensed Matter 22.3, p. 033101. URL: http://stacks . iop.org/0953-8984/22/i= 3/a=033101 (cit. on p. 37).

Herminghaus, S. (2005). "Dynamics of wet granular matter." In: Advances in Physics 54.3, pp. 221-261. DOI: 10.1080/00018730500167855. eprint: http : / / dx . doi . org/10 . 1080/00018730500167855. URL: http://dx. doi.org/10.1080/00018730500167855 (cit. on p. 18).

Heussinger, Claus (2013). "Shear thickening in granular suspensions: Interparticle friction and dynamically correlated clusters." In: Phys. Rev. E 88 (5), p. 050201. DOI: 10.1103/PhysRevE. 88.050201. URL: http://link.aps.org/doi/10.1103/PhysRevE.88.050201 (cit. on p. 17).

Heussinger, Claus, Pinaki Chaudhuri, and Jean-Louis Barrat (2010). "Fluctuations and correlations during the shear flow of elastic particles near the jamming transition." In: Soft Matter 6 (13), pp. 30503058. DOI: 10.1039/B927228C. URL: http://dx. doi.org/10.1039/ B927228C (cit. on p. 33).

Hornbaker D. J., Albert R., Albert I., Barabasi A.-L., and Schiffer P. (1997). "What keeps sandcastles standing?" In: Nature 387.6635, pp. 765-765. ISSN: 0028-0836 (cit. on p. 18).

Höhler, R and S Cohen-Addad (2005). "Rheology of liquid foam." In: Journal of Physics: Condensed Matter 17.41, Rio41. URL: http : //stacks . iop.org/0953-8984/17/i=41/a=R01 (cit. on p. 15).

Ikeda, Atsushi, Ludovic Berthier, and Peter Sollich (2012). "Unified study of glass and jamming rheology in soft particle systems." In: Phys. Rev. Lett. 109 (1), p. 018301. Dor: 10.1103/PhysRevLett. 109. 018301. URL: http : //link. aps .org/doi/10 . 1103/PhysRevLett . 109. 018301 (cit. on pp. 13-15,89).

- (2013). "Disentangling glass and jamming physics in the rheology of soft materials." In: Soft Matter 9 (32), pp. 7669-7683. DoI: 10. 
1039/C3SM50503K. URL: http://dx.doi.org/10. 1039/C3SM50503K (cit. on pp. 14, 89, 111).

Ilett, S. M., A. Orrock, W. C. K. Poon, and P. N. Pusey (1995). "Phase behavior of a model colloid-polymer mixture." In: Phys. Rev. E 51 (2), pp. 1344-1352. DOI: 10.1103/PhysRevE. 51.1344. URL: http: //link.aps.org/doi/10.1103/PhysRevE.51.1344 (cit. on p. 18).

Irani, Ehsan, Pinaki Chaudhuri, and Claus Heussinger (2014). "Impact of Attractive Interactions on the Rheology of Dense Athermal Particles." In: Phys. Rev. Lett. 112 (18), p. 188303. DoI: 10. 1103/PhysRevLett. 112.188303. URL: http://link.aps . org/doi/ 10.1103/PhysRevLett.112.188303.

- (2016). "Athermal rheology of weakly attractive soft particles." In: Phys. Rev. E 94 (5), p. 052608. Dor: 10.1103/PhysRevE. 94.052608. URL: http://link. aps.org/doi/10.1103/PhysRevE. 94.052608.

Irving, J. H. and John G. Kirkwood (1950). "The Statistical Mechanical Theory of Transport Processes. IV. The Equations of Hydrodynamics." In: The Journal of Chemical Physics 18.6, 817-829. Dor: 10.1063/1.1747782. URL: http://scitation . aip.org/content/ aip/journal/jcp/18/6/10.1063/1.1747782 (cit. on p. 22).

Jaeger, Heinrich M, Sidney R Nagel, and Robert P Behringer (1996). "Granular solids, liquids, and gases." In: Reviews of Modern Physics 68.4, p. 1259 (cit. on p. 27).

Kawasaki, Takeshi, Atsushi Ikeda, and Ludovic Berthier (2014). "Thinning or thickening? Multiple rheological regimes in dense suspensions of soft particles." In: EPL (Europhysics Letters) 107.2, p. 28009. URL: http : //stacks . iop . org/0295 - 5075/107/i=2/a=28009 (cit. on pp. $67,87,111$ ).

Knight, James B., H. M. Jaeger, and Sidney R. Nagel (1993). “Vibrationinduced size separation in granular media: The convection connection." In: Phys. Rev. Lett. 70 (24), pp. 3728-3731. Dor: 10.1103/ PhysRevLett. 70.3728. URL: http://link.aps.org/doi/10.1103/ PhysRevLett. 70.3728 (cit. on p. 105).

Lees, A W and S F Edwards (1972). "The computer study of transport processes under extreme conditions." In: Journal of Physics C: Solid State Physics 5.15, p. 1921. URL: http : //stacks . iop . org/0022 3719/5/i=15/a=006 (cit. on p. 21).

Lekkerkerker, H. N. W., W. C.-K. Poon, P. N. Pusey, A. Stroobants, and P. B. Warren (1992). "Phase Behaviour of Colloid + Polymer Mixtures." In: EPL (Europhysics Letters) 20.6, p. 559. URL: http : //stacks. iop.org/0295-5075/20/i=6/a=015 (cit. on p. 18).

Lieleg, O., M. M. A. E. Claessens, C. Heussinger, E. Frey, and A. R. Bausch (2007). "Mechanics of Bundled Semiflexible Polymer Networks." In: Phys. Rev. Lett. 99 (8), p. o88102. DoI: 10.1103/ PhysRevLett . 99 . 088102. URL: http : / / link. aps . org / doi / 10. 1103/PhysRevLett. 99.088102 (cit. on p. 38). 
Liu Andrea J. and Nagel Sidney R. (1998). “Nonlinear dynamics: Jamming is not just cool any more." In: Nature 396.6706. 10.1038/23819, pp. 21-22. ISSN: 0028-0836. DOI: http : //dx . doi . org/10 . 1038/ 23819 (cit. on pp. 11, 13, 14).

Liu, Andrea J. and Sidney R. Nagel (2010). "The Jamming Transition and the Marginally Jammed Solid." In: Annual Review of Condensed Matter Physics 1.1, pp. 347-369. DoI: 10 . 1146 / annurev conmatphys - 070909 - 104045. eprint: http : / / dx . doi . org / 10. 1146/annu rev - conmatphys - 070909 - 104045. URL: http: //dx. doi. org/10.1146/annurev - conmatphys - $070909-104045$ (cit. on p. 13).

Lois, Gregg, Jerzy Blawzdziewicz, and Corey S. O'Hern (2008). “Jamming Transition and New Percolation Universality Classes in Particulate Systems with Attraction." In: Phys. Rev. Lett. 100 (2), p. o280o1. DOI: 10 . 1103/PhysRevLett . 100 . 028001. URL: http : / / link . aps . org/doi/10.1103/PhysRevLett. 100.028001 (cit. on pp. 17, 18, 28, 30).

Mari, Romain, Florent Krzakala, and Jorge Kurchan (2009). "Jamming versus Glass Transitions." In: Phys. Rev. Lett. 103 (2), p. 025701. DOI: 10 . 1103/PhysRevLett . 103.025701. URL: http : // link . aps . org/doi/10.1103/PhysRevLett. 103.025701 (cit. on p. 13).

Martens, Kirsten, Lyderic Bocquet, and Jean-Louis Barrat (2012). "Spontaneous formation of permanent shear bands in a mesoscopic model of flowing disordered matter." In: Soft Matter 8 (15), pp. 41974205. DOI: 10 . 1039 / C2SM07090A. URL: http : / / dx . doi . org / 10 . 1039/C2SM07090A (cit. on p. 62).

Maxwell, J. Clerk (1864). "L. On the calculation of the equilibrium and stiffness of frames." In: Philosophical Magazine Series 4 27.182, pp. 294-299. DOI: 10 . 1080/14786446408643668. eprint: http : / / Www . tandfonline . com / doi / pdf / 10 . $1080 / 14786446408643668$. URL: http: //WWw. tandfonline. com/doi/abs/10.1080/14786446408643668 (cit. on pp. 12, 35).

Mitarai, Namiko and Franco Nori (2006). "Wet granular materials." In: Advances in Physics 55.1-2, pp. 1-45. DOI: 10. 1080/00018730600626065. eprint: http : / /dx . doi . org / 10 . 1080/00018730600626065. URL: http://dx.doi.org/10.1080/00018730600626065 (cit. on p. 18).

Moller, Peder, Abdoulaye Fall, Vijayakumar Chikkadi, Didi Derks, and Daniel Bonn (2009). "An attempt to categorize yield stress fluid behaviour." In: Philosophical Transactions of the Royal Society of London A: Mathematical, Physical and Engineering Sciences 367.1909, pp. 5139-5155. IssN: 1364-503X. DOI: 10 . 1098/ rsta. 2009. 0194 (cit. on pp. 17, 19, 30).

Nicolas, Alexandre, Jean-Louis Barrat, and Jörg Rottler (2016). "Effects of Inertia on the Steady-Shear Rheology of Disordered Solids." In: Phys. Rev. Lett. 116 (5), p. 058303. DoI: 10.1103/PhysRevLett. 116. 058303. URL: http://link. aps. org/doi/10.1103/PhysRevLett. 116.058303 (cit. on pp. 16, 63, 68). 
O'Hern, Corey S., Stephen A. Langer, Andrea J. Liu, and Sidney R. Nagel (2002). "Random Packings of Frictionless Particles." In: Phys. Rev. Lett. 88 (7), p. 075507. DoI: 10.1103/PhysRevLett. 88. 075507. URL: http : // link . aps .org/doi/10.1103/PhysRevLett. 88.075507 (cit. on p. 12).

O'Hern, Corey S., Leonardo E. Silbert, Andrea J. Liu, and Sidney R. Nagel (2003). "Jamming at zero temperature and zero applied stress: The epitome of disorder." In: Phys. Rev. E 68 (1), p. 011306. DOI: 10.1103/PhysRevE.68.011306. URL: http: // link. aps . org/ doi/10.1103/PhysRevE.68.011306 (cit. on pp. 12, 35).

Olmsted, PeterD. (2008). "Perspectives on shear banding in complex fluids." English. In: Rheologica Acta 47.3, pp. 283-300. IssN: 00354511. DOI: 10 . 1007 / s00397 - 008 - 0260 - 9. URL: http : / / dx . doi . org/10.1007/s00397-008-0260- 9 (cit. on p. 51).

Olsson, Peter and S. Teitel (2011). "Critical scaling of shearing rheology at the jamming transition of soft-core frictionless disks." In: Phys. Rev. E 83 (3), p. 030302. DOI: 10.1103/PhysRevE.83.030302. URL: http: / / link . aps . org/doi/10 . 1103/PhysRevE . 83.030302 (cit. on p. 33).

Ono, Ian K., Shubha Tewari, Stephen A. Langer, and Andrea J. Liu (2003). "Velocity fluctuations in a steadily sheared model foam." In: Phys. Rev. E 67 (6), p. 061503. DoI: 10 . 1103 / PhysRevE . 67. 061503. URL: http : // link . aps . org/doi/10 . 1103/PhysRevE . 67. 061503 (cit. on p. 50).

Otsuki, Michio and Hisao Hayakawa (2011). "Critical scaling near jamming transition for frictional granular particles." In: Phys. Rev. E 83 (5), p. 051301. DOI: 10.1103/PhysRevE.83.051301. URL: http: // link . aps . org/doi / 10 . 1103/PhysRevE . 83.051301 (cit. on p. 16).

Ovarlez, G., S. Rodts, X. Chateau, and P. Coussot (2009). "Phenomenology and physical origin of shear localization and shear banding in complex fluids." English. In: Rheologica Acta 48.8, pp. 831-844. ISSN: 0035-4511. DOI: 10 . 1007 / s00397 - 008 - 0344 - 6. URL: http : //dx. doi . org/10.1007/s00397-008-0344-6 (cit. on pp. 18, 53, 60).

Pham, K. N., A. M. Puertas, J. Bergenholtz, S. U. Egelhaaf, A. Moussard, P. N. Pusey, A. B. Schofield, M. E. Cates, M. Fuchs, and W. C. K. Poon (2002). "Multiple Glassy States in a Simple Model System." In: Science 296.5565, pp. 104-106. ISSN: 0036-8075. DOI: 10.1126/science. 1068238. eprint: http://science. sciencemag . org / content / 296/5565/104. full. pdf. URL: http : / science . sciencemag. org/content/296/5565/104 (cit. on p. 17).

Picard, Guillemette, Armand Ajdari, Lydéric Bocquet, and Fran çois Lequeux (2002). "Simple model for heterogeneous flows of yield stress fluids." In: Phys. Rev. E 66 (5), p. 051501. DoI: 10.1103/ 
PhysRevE . 66.051501. URL: http://link.aps . org/doi/10 . 1103/ PhysRevE.66. 051501 (cit. on pp. 19, 32, 52, 53).

Pierre-Gilles de Gennes ((1932-2007)). Pierre-Gilles de Gennes — Wikipedia, The Free Encyclopedia. URL: https : //en . wikipedia.org/w/index. php?title=Pierre-Gilles_de_Gennes\&oldid=721216608 (cit. on p. 3).

Rahbari, S. H. Ebrahimnazhad, J. Vollmer, S. Herminghaus, and M. Brinkmann (2010). "Fluidization of wet granulates under shear." In: Phys. Rev. E 82 (6), p. 061305. DOI: 10.1103/PhysRevE . 82. 061305. URL: http: //link. aps . org/doi/10 . 1103/PhysRevE . 82. 061305 (cit. on pp. 17, 30).

Rognon, Pierre G., Jean-noël Roux, Mohamed Naaïm, and François Chevoir (2008). "Dense flows of cohesive granular materials." In: Journal of Fluid Mechanics 596, pp. 21-47. ISsN: 1469-7645. DOI: 10. 1017/S0022112007009329. URL: http: // journals . cambridge . org/article_S0022112007009329 (cit. on p. 42).

Salerno, K. Michael and Mark O. Robbins (2013). "Effect of inertia on sheared disordered solids: Critical scaling of avalanches in two and three dimensions." In: Phys. Rev. E 88 (6), p. o62206. Dor: 10. 1103/PhysRevE . 88.062206. URL: http: // link. aps . org/doi/ 10.1103/PhysRevE.88.062206 (cit. on pp. 16, 63).

Salerno, K. Michael, Craig E. Maloney, and Mark O. Robbins (2012). "Avalanches in Strained Amorphous Solids: Does Inertia Destroy Critical Behavior?" In: Phys. Rev. Lett. 109 (10), p. 105703. Dor: 10. 1103/PhysRevLett . 109 . 105703. URL: http: / / link . aps . org/ doi/10.1103/PhysRevLett. 109. 105703 (cit. on pp. 16, 63).

Schall, Peter and Martin van Hecke (2010). "Shear Bands in Matter with Granularity." In: Annual Review of Fluid Mechanics 42.1, pp. 67-88. DoI: 10.1146/annurev - fluid - 121108 - 145544. eprint: http : / / dx . doi . org / 10 . 1146/annurev - fluid - 121108 - 145544. URL: http : / / dx . doi . org / 10 . 1146 / annurev - fluid - 121108 145544 (cit. on pp. 18, 53).

Shäfer, J., S. Dippel, and D. Wolf (1996). "Force Schemes in Simulations of Granular Materials." In: Journal de Physique I 6.1, pp. 520. DOI: 10 . 1051 / jp1 : 1996129. URL: https : / / hal . archives ouvertes. fr/jpa- 00247176 (cit. on p. 28).

Steinhardt, Paul J., David R. Nelson, and Marco Ronchetti (1983). "Bond-orientational order in liquids and glasses." In: Phys. Rev. B 28 (2), pp. 784-805. DOI: 10.1103/PhysRevB. 28.784. URL: http: //link.aps.org/doi/10.1103/PhysRevB.28.784 (cit. on p. 106).

Tewari, Shubha, Dylan Schiemann, Douglas J. Durian, Charles M. Knobler, Stephen A. Langer, and Andrea J. Liu (1999). "Statistics of shear-induced rearrangements in a two-dimensional model foam." In: Phys. Rev. E 6o (4), pp. 4385-4396. DoI: 10.1103/ PhysRevE . 60 . 4385. URL: http : / / link . aps . org/doi/10 . 1103/ PhysRevE.60.4385 (cit. on p. 67). 
Trulsson, Martin, Bruno Andreotti, and Philippe Claudin (2012). "Transition from the Viscous to Inertial Regime in Dense Suspensions." In: Phys. Rev. Lett. 109 (11), p. 118305. DoI: 10.1103/PhysRevLett. 109. 118305. URL: http:// /ink. aps . org/doi/10.1103/PhysRevLett. 109.118305 (cit. on pp. 67, 84).

Vågberg, Daniel, Peter Olsson, and S. Teitel (2014). "Dissipation and Rheology of Sheared Soft-Core Frictionless Disks Below Jamming." In: Phys. Rev. Lett. 112 (20), p. 208303. DoI: 10.1103/PhysRevLett. 112. 208303. URL: http://link. aps . org/doi/10.1103/PhysRevLett. 112. 208303 (cit. on pp. 15, 63, 66, 70).

Vågberg, Daniel, Peter Olsson, and S. Teitel (2014). "Universality of Jamming Criticality in Overdamped Shear-Driven Frictionless Disks." In: Phys. Rev. Lett. 113 (14), p. 148002. DoI: 10.1103/PhysRevLett. 113. 148002. URL: http://link. aps . org/doi/10.1103/PhysRevLett. 113.148002 (cit. on p. 36).

Wagner, Norman J. and John F. Brady (2009). "Shear thickening in colloidal dispersions." In: Physics Today 62.10, pp. 27-32. Dor: http: //dx.doi.org/10.1063/1.3248476. URL: http://scitation.aip. org/content/aip/magazine/physicstoday/article/62/10/10. 1063/1.3248476 (cit. on p. 9).

Wyart, M., H. Liang, A. Kabla, and L. Mahadevan (2008). "Elasticity of Floppy and Stiff Random Networks." In: Phys. Rev. Lett. 101 (21), p. 215501. DOI: 10.1103/PhysRevLett . 101.215501 . URL: http: //link.aps.org/doi/10.1103/PhysRevLett.101.215501 (cit. on p. 38).

Yerushalmi, Joseph, Stanley Katz, and Reuel Shinnar (1970). “The stability of steady shear flows of some viscoelastic fluids." In: Chemical Engineering Science 25.12, 1891-1902. ISSN: 0009-2509. DOI: 10. 1016/0009 - 2509 ( 70 ) 87007 - 5. URL: http : / /www. sciencedirect. com/science/article/pii/0009250970870075 (cit. on p. 19). 



\section{COLOPHON}

This document was typeset using the typographical look-and-feel classicthesis developed by André Miede. The style was inspired by Robert Bringhurst's seminal book on typography "The Elements of Typographic Style". classicthesis is available for both $\mathrm{LTT}_{\mathrm{E}} \mathrm{X}$ and $\mathrm{LYX}$ :

https://bitbucket.org/amiede/classicthesis/

Happy users of classicthesis usually send a real postcard to the author, a collection of postcards received so far is featured here:

http://postcards.miede.de/

Final Version as of December 8, 2016 (classicthesis). 